Karin Aguado / Lena Heine / Karen Schramm (Hrsg.)

\title{
Introspektive Verfahren und Qualitative Inhaltsanalyse in der Fremdsprachenforschung
}

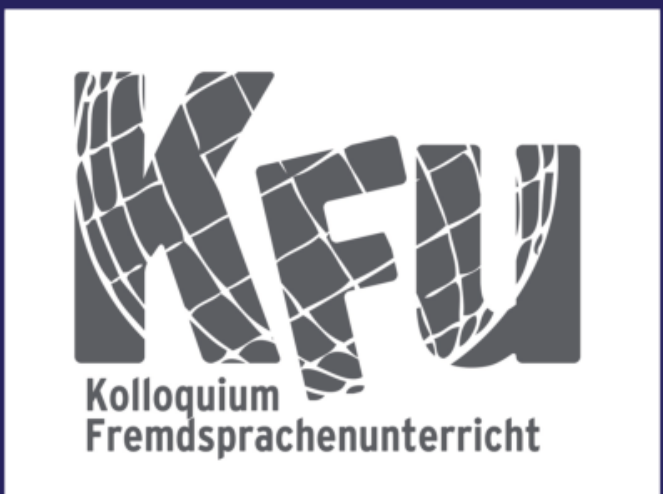


Introspektive Verfahren und Qualitative Inhaltsanalyse in der Fremdsprachenforschung 


\section{KOLLOQUIUM FREMDSPRACHENUNTERRICHT \\ Herausgegeben von Daniela Caspari, Lars Schmelter, Karin Vogt und Nicola Würffel}

\section{BAND 48}

Zu Qualitätssicherung und Peer Review der vorliegenden Publikation

Die Qualität der in dieser Reihe erscheinenden Arbeiten wird vor der Publikation durch alle vier Herausgeber der Reihe geprüft.
Notes on the quality assurance and peer review of this publication

Prior to publication, the quality of the work published in this series is reviewed by all four editors of the series. 
Karin Aguado / Lena Heine / Karen Schramm (Hrsg.)

\section{Introspektive Verfahren und Qualitative Inhaltsanalyse in der Fremdsprachenforschung}




\title{
Bibliografische Information der Deutschen Nationalbibliothek
}

Die Deutsche Nationalbibliothek verzeichnet diese Publikation in der Deutschen Nationalbibliografie; detaillierte bibliografische Daten sind im Internet über http:/ / dnb.d-nb.de abrufbar.

\author{
Umschlaglogo: \\ Christoph Baum
}

Die Herausgeberinnen danken der Universität Leipzig für die Übernahme eines Druckkostenanteils.

ISSN 1437-7829

ISBN 978-3-631-64057-9 (Print)

ISBN 978-3-653-02548-4 (E-Book)

DOI 10.3726/978-3-653-02548-4

\author{
(C) Peter Lang GmbH \\ Internationaler Verlag der Wissenschaften \\ Frankfurt am Main 2013 \\ Alle Rechte vorbehalten.
}

Peter Lang Edition ist ein Imprint der Peter Lang GmbH.

Peter Lang - Frankfurt am Main · Bern · Bruxelles · New York ·

Oxford $\cdot$ Warszawa $\cdot$ Wien

Das Werk einschließlich aller seiner Teile ist urheberrechtlich geschützt. Jede Verwertung außerhalb der engen Grenzen des

Urheberrechtsgesetzes ist ohne Zustimmung des Verlages unzulässig und strafbar. Das gilt insbesondere für Vervielfältigungen, Übersetzungen, Mikroverfilmungen und die Einspeicherung und Verarbeitung in elektronischen Systemen. www.peterlang.de 


\section{Inhalt}

Karen Schramm

Introspektion meets Qualitative Inhaltsanalyse - zur Einführung. 7

\section{Themenbereich Introspektive Verfahren}

\section{Lena Heine}

Introspektive Verfahren in der Fremdsprachenforschung:

State-of-the-Art und Desiderata

Petra Knorr

Zur Differenzierung retrospektiver verbaler Daten:

Protokolle Lauten Erinnerns erheben, verstehen und analysieren

Diana Feick

„Sehen Sie sich Ines an.“

Zur sozialen Situiertheit des Videobasierten Lauten Erinnerns

Ulrike Arras

Introspektive Verfahren in der Sprachtestforschung

Anna Katharina Schnell

Lautes Denken im Licht der Embodied Cognition:

neue Grenzen, neue Möglichkeiten in der Schreibprozessforschung?

\section{Themenbereich Qualitative Inhaltsanalyse}

\section{Karin Aguado}

Die Qualitative Inhaltsanalyse in der empirischen Fremdsprachenforschung:

Grenzen, Potentiale, Desiderata

Michaela Gläser-Zikuda

Qualitative Inhaltsanalyse in der Bildungsforschung -

Beispiele aus diversen Studien.

Jan-Oliver Eberhardt

Die qualitative Inhaltsanalyse als Interpretationsverfahren

zur Generierung eines empirischen Modells interkultureller Kompetenz

Michael Rogge

Die qualitative Inhaltsanalyse als Mittel zur empirischen Konkretisierung fremdsprachendidaktischer Paradigmata am Beispiel der inter- bzw.

transkulturellen Kompetenzen in fremdsprachlichen Begegnungsprojekten 
Annegret Beier

Die qualitative Inhaltsanalyse als transdisziplinäres Verfahren zur Auswertung von Leseprotokollen

Autorinnen und Autoren

Diskutantinnen und Diskutanten 


\section{Introspektion meets Qualitative Inhaltsanalyse - zur Einführung}

\section{Karen Schramm}

Forschungsmethoden stehen in der Fremdsprachendidaktik derzeit hoch im Kurs. Als Handwerkszeug sind sie in Zeiten immer komplexerer Forschungsdesigns und immer differenzierterer Erhebungs- und Analyseverfahren gleichermaßen für den wissenschaftlichen Nachwuchs wie auch für erfahrene Fremdsprachendidaktikerinnen und -didaktiker von größter Bedeutung. Entsprechend hoch sind das Bedürfnis nach Austausch und der Bedarf an wissenschaftlichen Publikationen zu diesem Themenfeld.

Die dritte Novembertagung 2011 am Herder-Institut der Universität Leipzig setzte deshalb die anregenden Forschungsmethodischen Gespräche der vergangenen Jahre in bewährter Weise fort: Anhand konkreter Forschungserfahrungen diskutierten Wissenschaftlerinnen und Wissenschaftler unterschiedlicher Qualifikationsstufen sprachdisziplinenübergreifend die besonderen Chancen und Grenzen ausgewählter Forschungsmethoden für die Fremdsprachendidaktik. In diesem Fall trafen die Themenschwerpunkte Introspektive Verfahren und Qualitative Inhaltsanalyse aufeinander - eine gute Mischung, wie sich in den lebhaften Diskussion herausstellte, die nicht zuletzt von den kritischen Impulsen der Diskutantinnen und Diskutanten Monika Geist, Susanne Göpferich, Adelheid $\mathrm{Hu}$, Henning Rossa und Eva Wilden ausgelöst wurden und für die an dieser Stelle im Namen aller Beteiligten noch einmal herzlich gedankt sei.

Nicht zuletzt dank der finanziellen Unterstützung durch die Deutsche Gesellschaft für Fremdsprachenforschung, die Universität Leipzig und die Reihe Kolloquium Fremdsprachenunterricht sind die Ergebnisse der Forschungsmethodischen Gespräche 2011 im vorliegenden Sammelband nun für weitere Interessierte dokumentiert. Die zehn Beiträge, die im Folgenden kurz vorgestellt werden, gliedern sich in zwei Teile: Im ersten Teil werden zunächst die introspektiven Verfahren thematisiert, der zweite Teil ist der Qualitativen Inhaltsanalyse gewidmet.

In ihrem einführenden Beitrag zu Teil 1 gibt Lena Heine einen Überblick über den aktuellen Forschungsstand der methodisch-methodologischen Diskussion zu introspektiven Verfahren in der Fremdsprachenforschung. Als neue Entwicklungen in diesem Feld hebt sie u. a. die Konsequenzen des Paradigmenwechsels von kognitiven zu soziokulturellen Theorien für die Erhebung und Analyse von Daten Lauten Denkens und Lauten Erinnerns hervor. Auch legt Heine für das dringende Desiderat, die Mehrsprachigkeit laut denkender Personen künftig auf theoretischer Ebene in die Methodendiskussion einzubeziehen, erste richtungs- 
weise Überlegungen vor, die sicherlich die weitere Diskussion zur Modellbildung bezüglich mehrsprachiger Lautdenkprozesse inspirieren werden.

Petra Knorr thematisiert in ihrem Beitrag zu Protokollen Lauten Erinnerns die Vielschichtigkeit retrospektiver verbaler Daten. An videographierten Beispielen dyadischer Planungsgespräche angehender Englischlehrpersonen zeigt sie anschaulich die Problematik auf, dass die Forschungspartner nach der Aufforderung zum Lauten Erinnern sowohl erinnerte Gedanken an die Handlung (in-action thoughts) als auch postaktionale Gedanken (hindsight thoughts) äußern. Diesbezüglich leistet ihr Beitrag nicht nur eine terminologische Systematisierung und Differenzierung retrospektiver verbaler Daten, sondern verdeutlicht insbesondere, dass die Analyse solcher Daten ihrer Beschaffenheit - einschließlich ihrer sozialen Bedingtheit und Adressiertheit - gerecht zu werden trachten sollte.

Die Frage nach der sozialen Situiertheit videobasierten Lauten Erinnerns vertieft Diana Feick, indem sie an transkribierten Beispielen aus einem in Mexiko durchgeführten Deutsch-als-Fremdsprache-Handyprojekt die Adressiertheit verbaler und nonverbaler Erinnerungsäußerungen aufzeigt. Obwohl dieses Phänomen aus kognitiver Forschungsperspektive allgemein als zu vermeiden gilt, trat es in der konkreten Studie trotz entsprechenden vorsorglichen Trainings und trotz Abwesenheit der Forscherin auf. Feick nimmt diese Befunde zum Anlass, aus soziokultureller Perspektive die Reaktivität der Datenerhebung durch videobasiertes Lautes Erinnern und Fragen einer kontextadäquaten Analyse solcher Daten zu diskutieren.

Ulrike Arras wendet sich in ihrem Beitrag dem Einsatz introspektiver Verfahren und insbesondere des Lauten Denkens in der traditionell quantitativ ausgerichteten Testforschung zu. In diesem Themenfeld können introspektive Verfahren zum einen zur Erforschung lernerseitiger Teststrategien und zum anderen zu einem genaueren Verständnis prüferseitiger Beurteilungsstrategien einen wesentlichen Beitrag leisten. Besonders vielversprechendes Potential introspektiver Verfahren für die Testforschung erkennt Arras nicht nur darin, dass sie der Überprüfung der Validität von Tests dienen; auch bei der Prüferschulung und beim Testtraining für Lernende lassen sie sich gewinnbringend einsetzen, um die Reflexionsfähigkeit der Betroffenen zu steigern.

Das Laute Denken beleuchtet auch Anna Katharina Schnell. Sie verbindet neuere Ansätze aus der Kognitionspsychologie mit der Methodik und zeigt bei ihrem kritischen Zugriff zahlreiche Grenzen des Verfahrens auf. Als konkreter Ausgangspunkt dienen ihr Laut-Denk-Daten von deutschen Französischlernern während der zweitsprachlichen Textproduktion, um aus der Perspektive der Embodied und Distributed Cognition insbesondere nach einem möglichen Einbezug emotionaler, situationaler und sozialer Prozesse in die Datenanalyse zu fragen. 
Mit ihrem Beitrag zu Grenzen, Potentialen und Desiderata in Bezug auf die Qualitative Inhaltshaltsanalyse in der empirischen Fremdsprachenforschung eröffnet Karin Aguado den zweiten Themenbereich. Sie fragt zunächst nach den Gründen für die Beliebtheit dieser Analysemethode und gibt einen kurzen Überblick über die historische Entwicklung und zentrale Merkmale der Qualitativen Inhaltsanalyse. Bei ihrem anschließenden Überblick über aktuelle fremdsprachendidaktische Studien, die sich der Qualitativen Inhaltsanalyse bedienen, beleuchtet sie die besonderen Chancen und Grenzen des Verfahrens und bestimmt aus der Perspektive der Fremdsprachenforschung spezifische methodologische Desiderata.

Ergänzt wird dieser Überblick von Michaela Gläser-Zikudas grundlegendem Beitrag, der anhand von Beispielen aus den Erziehungswissenschaften und der Fremdsprachendidaktik in Techniken und Analyseschritte der Qualitativen Inhaltsanalyse einführt und dabei auch zweckdienliche Computerprogramme vorstellt. Als Gütekriterien Qualitativer Inhaltsanalyse beleuchtet sie Verfahrensdokumentation, Regelgeleitetheit, Intercoder-Reliabilität, Kommunikative Validierung und die Kombination qualitativer und quantitativer Analyseschritte genauer.

Nach diesen beiden Einführungen thematisieren drei Beiträge die Chancen und Grenzen der Qualitativen Inhaltsanalyse an konkreten fremdsprachendidaktischen Forschungsprojekten. Jan-Oliver Eberhardt nutzt sein empirisches Forschungsprojekt zur Weiterentwicklung eines Strukturmodells interkultureller Kompetenz als Grundlage, um konkrete Einblicke in sein inhaltsanalytisches Auswertungsverfahren zu gewähren. Bezogen auf Fragebogen- und Gruppeninterviewdaten von Schülerinnen und Schüler der 10. Klasse, die Französisch lernen, arbeitet er als wesentliche Stärke des Verfahrens u. a. die Kombination deduktiver und induktiver Auswertung heraus.

Ebenfalls mit Blick auf die interkulturelle kommunikative Kompetenz geht Michael Rogge am Untersuchungsgegenstand von Austausch- und Begegnungsprojekten der methodischen Fragestellung nach, wie der spezifische Entstehungskontext der von ihm erhobenen Schülerdokumentationen, die Subjektivität und die Prozesshaftigkeit der interkulturellen Lernerfahrungen bei der Qualitativen Inhaltsanalyse in angemessener Weise berücksichtigt werden können. Nach einer differenzierten Auseinandersetzung mit den Gütekriterien Qualitativer Inhaltsanalyse nimmt er eine domänenspezifische Anpassung des Auswertungsverfahren von Mayring (2008) vor, die die Einführung von Kontrollschritten vorsieht und einen iterativen Charakter aufweist.

Annegret Beier lenkt abschließend unseren Blick auf Einsatzmöglichkeiten der Qualitativen Inhaltsanalyse bei visuellen Lernerdaten. Am Beispiel einer literaturdidaktischen Deutsch-als-Fremdsprache-Unterrichtssequenz mit usbekischen Studierenden diskutiert sie Auswertungsverfahren in Bezug auf visuelle 
Leseprotokolle, die sie in ihrer Studie mit Interviewdaten trianguliert. Bei ihrer konkreten Illustration der Analyseschritte steht insbesondere die Frage nach den medialen Besonderheiten im Vordergrund der methodischen Reflexion. 
I. Themenbereich Introspektive Verfahren 



\title{
Introspektive Verfahren in der Fremdsprachenforschung: State-of-the-Art und Desiderata
}

\section{Lena Heine}

\begin{abstract}
This article follows three aims: the first is to present a short state-of-the-art of the use and methodological discussion on introspective methods in SLA with a special focus on research from Germany. The second is to map important new developments; in particular, the theoretical shift from cognitive to sociocultural frameworks and its consequences for the methodology, the focus on emotions in simultaneous think-aloud protocols (alongside with the introduction of discourse analytical methods to the analysis of think-aloud protocols) as well as the rise of consciousness in the field that individual verbal modes of representation play an important role for the use of verbal protocols as data. In the third section, desiderata in the theoretical discussion of the methodology are addressed, especially those concerning the multilingual nature of introspection in L2 research, and to suggest starting points for further research that take recent psycholinguistic models of multilingual language processing and models of the multilingual mental lexicon into account.
\end{abstract}

\section{Einleitung}

Introspektive Verfahren der Datenerhebung, insbesondere in Form von Lautem Denken und retrospektiven Interviews, gehören in der Fremdsprachenforschung zum Standardrepertoire empirischer Forschung und werden z. B. in einer Fülle von kürzlich entstandenen bzw. noch im Entstehen begriffenen deutschsprachigen Qualifikationsarbeiten verwendet (z. B. Behrend in Vorbereitung, Diao 2010, Feick in Vorbereitung, Ishitsuka in Vorbereitung, Knorr in Vorbereitung, Matias in Vorbereitung, Shafer 2011, Zawadzka 2011).

Neue Entwicklungen im Bereich der Fremdsprachenforschung und der Kognitionspsychologie sowie die Tatsache, dass wichtige Erkenntnisse aus der Erforschung individueller Mehrsprachigkeit bisher noch gar nicht oder noch nicht zufriedenstellend mit der Theoriebasis introspektiver Verfahren verknüpft worden sind, lassen es notwendig erscheinen, den jetzigen Forschungsstand zu introspektiven Verfahren in der Fremdsprachenforschung zusammenzufassen und mit einer kritischen metatheoretischen Reflexion zu verbinden.

Weil zentrale Impulse hierzu aus der deutschsprachigen Forschung stammen, wird im Folgenden auch vor allem auf diese fokussiert. Nach einer begrifflichen Klärung wird zunächst der jetzige Forschungsstand umrissen, bevor auf neuere Entwicklungen im Gebiet der Introspektionsforschung eingegangen wird. Abschließend werden Desiderata angesprochen, die so noch gar nicht im Diskurs verankert sind, aber dringend einer weiteren Erforschung bedürfen. 


\section{Der Introspektionsbegriff in der Fremdsprachenforschung}

Unter introspektiven Verfahren kann man nach einem weiten Verständnis jegliche Form von Erhebungsverfahren verstehen, durch die Versuchspersonen Einblicke in mentale Abläufe gewähren, die der direkten Beobachtung unzugänglich sind; hierunter fallen z. B. auch Interviewdaten und Tagebucheinträge (s. z. B. Ericsson/Simon 1993: 49ff und Heine 2005 für eine detaillierte Terminologiediskussion). Allerdings hat sich in der Fremdsprachenforschung mittlerweile eher ein stärker abgegrenztes Verständnis etabliert, das vor allem Lautes Denken und retrospektive Interviews unter introspektive Verfahren subsummiert.

Als prototypische Form einer introspektiven Methode kann dabei das Laute Denken bezeichnet werden. Es basiert auf Annahmen der Informationsverarbeitung und Problemlösetheorie und wurde grundlegend von Ericsson/Simon (1993) ausgearbeitet. Die dieser Methode unterliegende Grundannahme ist die, dass Versuchspersonen ihre Gedanken verbalisieren können, ohne dass die Verbalisierung den Gedankenverlauf beeinflusst. Lautes Denken macht sich also die Tatsache zunutze, dass Gedanken vom denkenden Subjekt selbst verbalisiert werden können, so dass diese Gegenstand einer Erforschung werden können. Hierbei wird angenommen, dass Gedankenaktivität als eine Abfolge mentaler Zustände betrachtet werden kann, und dass viele Elemente einer solchen Gedankenfolge spontan und ohne zusätzlichen kognitiven Aufwand mit einer verbalen Form assoziiert werden, die normalerweise als inner speech unvokalisiert bleibt. Die Annahme ist nun, dass es prinzipiell möglich ist, diese verbalen Gedanken laut auszusprechen (wobei nicht zum Zweck der Kommunikation an ein Gegenüber oder an sich selbst gerichtete Sprache im Sinne von private speech, sondern unreflektiertes lautes Mitvokalisieren ablaufender Gedanken gemeint ist). Dadurch wird ein Strom spontan geäußerter und häufig fragmentarischer und syntaktisch unverbundener verbaler Daten produziert, aus denen dann rekonstruiert werden kann, worauf die Versuchsperson zu einem gegebenen Zeitpunkt jeweils ihre Aufmerksamkeit gerichtet hat. Mit ihrer Hilfe werden beispielsweise Testerstrategien (Arras 2009, s. auch Arras in diesem Band); Übersetzungsprozesse (Göpferich 2006, Schnell in Vorbereitung); Strategien beim Aufgabenlösen (Heine 2010, Würffel 2006); Prozesse des Hörverstehens (Rossa 2012) oder Leseverstehen (Schramm 2001) untersucht.

Die Tatsache, dass Äußerungen simultan zur zu untersuchenden Handlung erhoben werden, macht die Erhebung von Lautdenkprotokollen zu einem anfälligen Verfahren, da ein unsachgemäßer Einsatz sich reaktiv auf die Gedankengänge auswirkt. Insbesondere ist bekannt, dass eine Adressierung der Äußerungen sowie das Bemühen, sich verständlich $\mathrm{zu}$ machen, zu verstärkter mentaler Konstruktionsaktivität führen und damit mentale Abläufe beeinflussen (vgl. Ericsson/Simon 1993 für eine detaillierte Diskussion). Daher bedarf die Methodik eines genauen theoretischen Verständnisses und eines rigiden Erhe- 
bungsprozederes (Heine erscheint, Heine/Schramm 2007), um auch tatsächlich Daten generieren zu können, die valide Aussagen über die zu untersuchenden Abläufe erlauben. Im Rahmen einer Datenerhebung werden Versuchspersonen zur Vermeidung reaktiver Effekte daher meist gezielt dahingehend trainiert, so viel ihrer Gedanken zu vokalisieren wie möglich, während sie sich nicht auf die Verbalisierung, sondern voll auf die auszuführende Tätigkeit konzentrieren, und nur das laut aussprechen, was ihnen automatisch in den Kopf kommt.

Lautes Denken muss wegen dieser Spezifika mit anderen Formen verbaler Daten im Kontrast gesehen werden, wie sie etwa durch Interviews und Fragebögen erhoben werden, obwohl letztere ebenfalls introspektive Charakteristika aufweisen. Ein gewichtiger Unterschied liegt jedoch darin, dass in Interviewund Fragebogendaten im Normalfall immer eine selbstreflexive Position eingenommen wird, der generelles Wissen und Selbstwahrnehmung unterliegt. Antworten auf Fragebogen- und Interviewfragen sind daher immer durch das Selbstbild der Versuchsperson, Annahmen über Erwartungen und soziale Erwünschtheit und den mehr oder weniger bewussten Versuch geprägt, sein eigenes Verhalten stimmig in einen gut motivierten und kohärenten Zusammenhang einzuordnen. Interview- und Fragebogendaten können daher interessante Informationen über Wissensbestandteile, generellen Strategiegebrauch etc. liefern, sind allerdings nicht dazu geeignet, individuelle mentale Prozesse abzubilden.

Retrospektive Verfahren versuchen ebenfalls, Einblick in konkret ablaufende Prozesse während einer Tätigkeit zu nehmen, versuchen jedoch die Problematik der Reaktivität zu umgehen, indem sie erst im Anschluss an die auszuführende Tätigkeit, z. B. durch Videostimuli, den Moment der Ausführung in den Blick nehmen. Versuchspersonen werden daher dazu angehalten, sich wieder in den jeweiligen Augenblick zurückzuversetzen und laut auszusprechen, was ihnen dabei durch den Kopf ging (Gass/Mackey 2000, Feick in diesem Band, Knorr in diesem Band). Retrospektive Interviews finden in einer Fülle von Arbeiten Verwendung, dienen aber selten als Hauptdatenquelle, sondern werden meist zu Triangulierungszwecken zusätzlich zu anderen Datensätzen wie etwa direkten Beobachtungsdaten eingesetzt (z. B. in Arras 2007, Dahnken 2005, Eckerth 2003, Lindemann 2009, Morkötter 2005, Raith 2011, Reinhold 2011).

Zusammenfassend lässt sich damit festhalten, dass die in der Fremdsprachenforschung sich etablierende Bedeutungsverengung auf Lautes Denken und retrospektives „Lautes Erinnern“ (Knorr in diesem Band, Knorr/Schramm 2012) sich in folgenden Merkmalen introspektiver Daten manifestiert: 
Introspektive Daten

- haben im Wesentlichen eine verbale Form;

- sollen Gedankenverläufe bezogen auf eine konkrete Tätigkeit abbilden, die es zu untersuchen gilt, ohne den Verlauf dieser Tätigkeit selbst zu beeinflussen (z. B. das Schreiben eines L2-Textes, das Lösen einer Übersetzungsaufgabe, das Bewertungsverhalten von Sprachstandstestern usf.);

- stammen von der zu untersuchenden Person selbst;

- lassen kleinschrittige und detaillierte Einsichten in mentale Abläufe zu.

\section{Was ist der bisherige Stand der Methodikdiskussion?}

Die methodologische Diskussion introspektiver Verfahren ist in der deutschsprachigen Fremdsprachenforschung in den vergangenen zehn Jahren vor allem durch Abgrenzungsversuche, eine Präzision von Begrifflichkeiten und eine grundlegende Validitätsdiskussion geprägt gewesen, die schon vorher begonnen wurde (z. B. Würffel 2001), aber vor allem seit Mitte der 2000er Jahre in einer Reihe von Überblicksartikeln (Aguado 2004, Beyer 2005, Heine 2005, Heine/ Schramm 2007, zur Begriffsschärfung auch v.a. Knorr in diesem Band), aber auch einer metaanalytischen Monographie zum Lauten Denken (Bowles 2010) noch weiter präzisiert werden konnte.

Die bisher die Methodikdiskussion bestimmende Frage ist die nach der Validität introspektiver Verfahren. Ericsson/Simon (1993) nähern sich dieser Frage theoretisch wie empirisch, setzen sich detailliert mit kritischen Stimmen auseinander und kommen zu dem Schluss, dass bei genauer Planung und korrekt durchgeführtem Probandentraining keine Reaktivität bei simultaner Erhebung von Verbalprotokollen anzunehmen sei. Bowles (2010) kommt diesbezüglich in ihrer Metaanalyse, mit der die Frage nach der Reaktivität abschließend zu beantworten versucht wird, zwar zu dem Schluss, dass Lautes Denken in Bezug die Aufgabenbearbeitung weniger reaktiv sei als retrospektive Verbalisierungen und sich insgesamt eine gemäßigte Reaktivität nicht-metakognitiver Lautdenkprotokolle u. a. in Bezug auf verbesserte Lernleistungen zeige, allerdings bedienen sich die Studien, die in die Metaanalyse eingeflossen sind, höchst unterschiedlicher Aufgabenformen, kontrollieren potentiell einflussnehmende Variablen kaum und sind damit schwer miteinander vergleichbar.

Insgesamt lässt sich zum jetzigen Zeitpunkt zumindest festhalten, dass die terminologische Unschärfe, die noch in den 90er Jahren in Bezug auf ein genaues Verständnis insbesondere von Lautem Denken und den einflussnehmenden Parametern auszumachen war, mittlerweile als ausgeräumt gelten kann. Es gibt darüber hinaus nun genügend leicht verständliche Einführungen und Handreichungen (s. o. g. Überblicksartikel), die auch auf den praktischen 
Einsatz in Erhebungssituationen in L2-Kontexten vorbereiten und damit eine theoretisch rigide Datenerhebung ermöglichen (Heine/Schramm 2007, Heine erscheint). Insgesamt fällt dabei auf, dass dem Lauten Denken in der Methodikdiskussion weitaus mehr Raum zugesprochen wird als retrospektiven Verfahren, deren aktueller Diskussionsstand noch im Wesentlichen durch die Monographie von Gass/Mackey (2000) bestimmt ist. Die Beiträge von Knorr und Feick im vorliegenden Band sind nicht zuletzt aus diesem Grund richtungsweisend, denn sie bringen neue Aspekte zur Sprache, die u. a. die makrotheoretische Einbettung von retrospektiven Verfahren betreffen. Diese sollen im nächsten Unterkapitel genauer beleuchtet werden.

\section{Neuere Entwicklungen in der Diskussion introspektiver Verfahren}

\subsection{Soziokulturelle Theorie als neue Makrotheorie}

Eine Art Paradigmenwechsel zeichnet sich in der Fremdsprachenforschung durch den Wechsel von eher kognitiv ausgerichteten Annahmen zum soziokulturellen bzw. soziokognitiven Paradigma ab. Sprache und Sprachenlernen werden nunmehr weniger als rein kognitive Abläufe betrachtet, sondern immer im Zusammenhang ihrer sozialen Funktion und Einbettung gesehen. Damit gewinnen Konstrukte wie Adressatenorientierung, Selbstbild und eigenes Rollenverständnis an Bedeutung.

Für die Verwendung von introspektiven Verfahren hat dies gewichtige Konsequenzen. Zumindest der Fall des Lauten Denkens setzt wie oben beschrieben voraus, dass es möglich ist, Gedanken laut auszusprechen, ohne diese dadurch zu verändern. Soziokulturelle und soziokognitive Verständnisse haben jedoch eine andere Sicht auf Sprache, indem sie davon ausgehen, dass Prozesse der Versprachlichung aufgrund der sozialen Vermitteltheit von Sprache grundsätzlich immer auf Gedanken rückwirken, da sie inhärent immer dialogisch, adressiert, und damit beziehungsstiftend und explikativ seien, was Prozesse des Verknüpfens und der Einsicht verursache, also reaktiv wirke. Sprache, so wird angenommen, hat damit per se schon immer eine primär interaktive weil kommunikative Funktion, die nicht unterdrückt werden kann. Ein soziokultureller Ansatz ist damit im Wesen nur schwer mit den Grundannahmen des simultanen, unreflektierten Verbalisierens nach Ericsson/Simon (1993) vereinbar.

Fragen nach der makrotheoretischen Einbettung und ihrer Konsequenzen werden so bisher noch nicht im Rahmen der Introspektionsdebatte problematisiert. Mit den Beiträgen von Feick und Knorr in diesem Band werden dazu erste Ansätze vorgelegt, die sich allerdings beide auf retrospektive Introspektion und besondere Aspekte der rückbezogenen Verbalisierungen beziehen. Inwiefern jedoch simultanes Lautes Denken im Rahmen eines soziokulturellen Theoriever- 
ständnisses als Erhebungsmethode überhaupt noch als valide betrachtet werden kann, bedarf aufgrund der starken momentanen Etablierung soziokultureller Modelle in der Fremdsprachenforschung dringend Beachtung. Bisher noch nicht mit soziokulturellen bzw. -kognitiven Annahmen verbunden ist beispielsweise die Frage, ob trotz der Grundannahme der sozialen Funktion von Sprache nicht doch eine automatische Assoziation von sprachlichen Formen mit still geäußerten Gedanken anzunehmen ist, diese also auch bei stiller Ausführung einer Tätigkeit normalerweise ablaufen. In diesem Fall wäre es ja in der Tat wenig sinnvoll, eine Reaktivität durch die Aktivierung einer sprachlichen Form anzunehmen, denn dann wären Gedanken, auch still gedachte, immer ,reaktiv', wodurch das Konstrukt überflüssig würde. Hier wäre zu klären, ob die oben genannten Effekte der Neustrukturierung und Verknüpfung lediglich durch die laute Vokalisierung ausgelöst werden, die wiederum zum eigenen sprachlichen Input wird, und wenn ja, warum das Hören der eigenen Stimme diese Effekte gegenüber einer stillen Bearbeitung auslösen sollte (s. ansatzweise hierzu Schnell in diesem Band).

\subsection{Emotionen}

In jüngerer Zeit wird in der Fremdsprachenforschung verstärkt auf die Rolle von Emotionen und Körperlichkeit beim Lernen, Lehren und Verwenden von Fremdsprachen verwiesen (u. a. Beermann/Cronjäger 2011, Börner/Vogel 2004, Ogasa 2011, Pavlenko 2005, 2006). Diese Entwicklung hat ebenfalls mit der Abkehr von rein kognitiv ausgerichteten Theorierahmen $\mathrm{zu}$ tun und trägt aktuellen kognitionstheoretischen Entwicklungen Rechnung. Bezüglich spezifischer Fragen, die sich auf die Erhebung von simultanen Lautdenkprotokollen bei Schreibaufgaben beziehen, wird dieser Aspekt durch den Beitrag von Schnell in diesem Band in die Diskussion eingeführt und detailliert behandelt. Neben einem Plädoyer für die Aufnahme emotionaler, situationer und sozialer Aspekte in Modelle der menschlichen Informationsverarbeitung, die der Erhebung von Lautdenkdaten unterliegen, schlägt sie auch eine Weiterentwicklung der Analyse Daten Lauten Denkens in Hinblick auf die Integration von diskursanalytischen Vorgehensweisen vor, die gezielt die in der Verbalisierung gewählten linguistischen Realisierungsvarianten in den Verbalprotokollen als Ausdruck multimodaler Wahrnehmung betrachtet, wie sie bei den Versuchspersonen in der Erhebungssituation evoziert werden. Durch eine stärkere Gewichtung der sprachlichen Form in der Auswertung, so zeigt sie anhand eigener Datenbeispiele, ist es möglich, u. a. auf handlungsbeeinflussende emotionale Abläufe rückzuschließen. 


\subsection{Individuelle Unterschiede in Bezug auf verbale Repräsentationsmodi}

Obwohl die Erkenntnis in der Fremdsprachenforschung nicht neu ist, dass sich eine Vielzahl an Faktoren und individuelle Prädisposition potentiell auf das Lernen und Verwenden von Fremdsprachen auswirken kann, also Bewusstsein darüber herrscht, dass sich Menschen z. B. als Lernertypen stark unterscheiden können, sind individuelle Aspekte kognitiver Typisierung bisher nur vereinzelt (Heine 2005, 2010) auf die Diskussion über introspektive Verfahren, insbesondere das Laute Denken, übertragen worden. Dieser Punkt ist wichtig, da er Auswirkungen sowohl auf die Frage nach der generellen Validität der Methode als auch auf forschungspraktische Entscheidungen hat.

Für die hier fokussierte Methodikdebatte ist besonders ein Aspekt individueller Verarbeitung von Relevanz, nämlich die Frage danach, inwiefern sich die Verknüpfung zwischen konzeptueller und sprachlicher Repräsentation individuell unterscheidet. So liefert beispielsweise Heine (2010) empirische Hinweise darauf, dass diese Verbindung individuell höchst unterschiedlich ausgeprägt sein kann, es eher verbale und eher nonverbale Denker (mit einer potentiellen Vielzahl an Abstufungen dazwischen) zu geben scheint. Während damit die Anforderung, die eigenen Gedanken unmittelbar laut auszusprechen, für manche Versuchspersonen als sehr natürlich und „leicht“ empfunden wird („,das mache ich immer, wenn ich alleine bin"), empfinden andere diese als unnatürliche und schwierige Anforderung (,ich würde nie so laut denken“), die auch potentiell mit kognitivem Aufwand und separaten Problemlöseschritten verbunden ist (Heine 2010). ${ }^{1}$ Dies hat unmittelbare Konsequenzen für die Validität von Lautdenkdaten, da nur für den eher verbalen Typus eine unmittelbare und automatisierte Verbalisierung von Gedankeninhalten angenommen werden kann. Im Grunde müsste vor einer Datenerhebung damit zunächst eine Typisierung von potentiellen Versuchspersonen durchgeführt werden und nur solche für die Erhebung simultaner Verbalprotokolle eingesetzt werden, denen die Verbalisierung wenig Mühe zu bereiten scheint. Dies ist meines Wissens bisher noch in keiner Studie geschehen.

\footnotetext{
${ }^{1}$ Zwar weisen auch Ericsson/Simon (1993: 250) darauf hin, dass individuelle Verarbeitungsunterschiede bestehen können und manche Versuchspersonen besser verbalisieren können als andere, ziehen daraus jedoch nicht die Konsequenz, dass dies ein Problem für die Validität darstellen könnte, sondern erwarten lediglich Auswirkungen auf die Verbalisierungs- und Aufgabenbearbeitungsgeschwindigkeit.
} 


\section{$4 \quad$ Modelle des mehrsprachigen mentalen Lexikons und Lautes Denken}

Obwohl Lautes Denken mit seinen starken Annahmen über die Verknüpfung zwischen Sprache und konzeptuellen Repräsentationen grundlegend auf psycholinguistischen Annahmen basiert, ist die Methodikdiskussion nie explizit mit dem Diskurs in diesem Forschungsfeld verknüpft worden; damit haben sich Weiterentwicklungen in der Psycholinguistik, die Erkenntnisse über die Speicherung und Aktivierung von Sprachwissen und den Zusammenhang zwischen Sprache und konzeptuellen Inhalten betreffen, auch nie auf die Methodikdiskussion niedergeschlagen; stattdessen wird noch immer das aus den 1980er Jahren stammende Verständnis von Ericsson/Simon (1993) operationalisiert (s. dazu ausführlich auch Schnell in diesem Band). Hier wäre eine aktualisierte Diskussion, die zeitgemäße Erkenntnisse der mehrsprachigen Sprachverarbeitung mit introspektiver Methodik in Zusammenhang bringt, wünschenswert.

Dazu kommt ein weiterer Aspekt: „Bilinguals, by definition are complex“ (Grosjean 1997: 226); die ohnehin schon hohe Komplexität individueller Unterschiede bei der Sprachverarbeitung wird bei mehrsprachigen Individuen in ihrer Faktorenvielfalt noch potenziert. Trotz dieser Einsicht haben die Besonderheiten mehrsprachiger Sprachverarbeitung bisher keinen Eingang in die Modellbildung für Lautes Denken gefunden. Stattdessen wird der prototypische Fall eines monolingualen Sprechers zugrunde gelegt, also ein vereinfachtes Sprachmodell übernommen, das auch in der Verwendung in der Fremdsprachenforschung bisher erstaunlicherweise kaum in Bezug auf seine Passung auf mehrsprachige Versuchspersonen in Frage gestellt worden ist. Auf der Grundlage eines solch vereinfachten Modells versucht beispielsweise die auf die L2-Spezifik ausgerichtete Monographie von Bowles (2010) Antworten auf die Frage zu geben, ob die Verwendung einer Fremdsprache als Verbalisierungssprache reaktive Effekte aufweist. Dabei vernachlässigt sie wie oben bereits erwähnt allerdings, dass wichtige Variablen wie der Sprachstand der untersuchten Personen oder die individuellen Kompetenzausprägungen in den L2-Teilfertigkeiten nicht kontrolliert werden, die potentiell Einfluss darauf nehmen, ob in der Erhebung valide Daten erhoben werden. Allerdings liegen aus der Forschung zum mehrsprachigen mentalen Lexikon empirische Belege dafür vor (z. B. De Groot/Poot 1997), dass unterschiedliche Kompetenzniveaus in der L2 zu unterschiedlichem Übersetzungsverhalten führen, ,suggesting different types of memory structures“ (ebd.: 218). Insbesondere zeigen kompetentere L2-Lerner Verhaltensweisen, die auf darauf schließen lassen, dass zwischen L2-Lexemen und der unterliegenden konzeptuellen Struktur starke Verknüpfungen bestehen, während weniger fortgeschrittene Lerner stärkere Verbindungen zwischen L1-und L2Wortform aufweisen als zwischen L2-Form und unterliegendem Konzept, also 
bei der Verwendung der L2 Übersetzungsprozesse über die L1 eingeschoben werden.

Ich will aus diesen Gründen im Folgenden ansprechen, dass die Frage danach, welche Konsequenzen es hat, ob in der L1 oder der L2 verbalisiert wird, mehr Probleme aufwirft, als es die bisherige Literatur suggeriert. Wahrscheinlich kann sie gar nicht abschließend beantwortet werden, da das Zusammenspiel von möglichen Einflussfaktoren zu komplex und individuell unterschiedlich wirkt, um pauschale Aussagen zu treffen; vielmehr bedarf sie genau durchdachter Positionen, die für die jeweilige Erhebungssituation und Population neu zu begründen sind.

Um diese Aspekte genauer zu diskutieren, ist es hilfreich, noch einmal von der bereits beschriebenen Annahme über die Verbalisierbarkeit von Gedanken von Ericsson/Simon (1993) auszugehen. Sie nehmen an, dass zumindest ein Teil unserer Gedanken als sequenzielle Abfolge mentaler Zustände beschrieben werden kann, die mit einer verbalen Repräsentation verknüpft ist, die dann vokalisiert werden kann (Abb. 1).

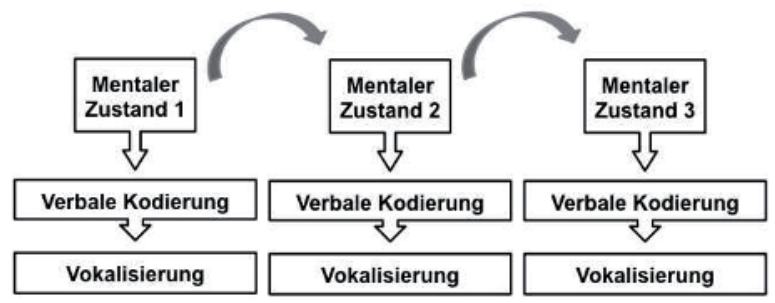

Abbildung 1: Schematische Darstellung von Lautem Denken (nach Ericsson/Simon 1987: 33)

Bei mehrsprachigen Sprechern wird nun angenommen, dass es keine Auswirkungen auf die Abfolge dieser mentalen Prozesse hat, welche ihrer Sprachen sie verwenden - solange sie gut genug beherrscht werden und den Versuchspersonen freigestellt wird, nach Belieben zwischen ihren Sprachen zu wechseln (vgl. Ericsson/Simon 1993: 249ff). Damit wird angenommen, dass beide Sprachsysteme mit einer einzigen unterliegenden konzeptuellen Repräsentation verbunden sind, wie man sie in Abbildung 2 darstellen könnte. Je nachdem, welches Einzelsprachenlexem gerade am stärksten mit dem jeweiligen Konzept verbunden ist, findet - so die Annahme - ein automatischer Sprachwechsel statt, der ohne zusätzliche Such- und Problematisierungsprozesse auskommt. 


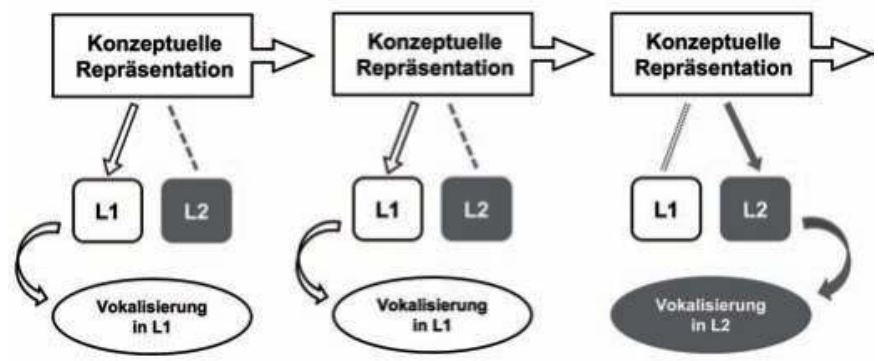

Abbildung 2: Grundmodell, das allgemeine Annahmen über die mehrsprachige Sprachverarbeitung beim Lauten Denken abbildet; unterliegende Annahme: Sprachwechsel sind frei möglich

Allerdings ist es durchaus problematisch, eine völlig ausgeglichene Sprachproduktion bei mehrsprachigen Individuen pauschal anzunehmen. Wie die folgenden Datenbeispiele aus Heine (2010) andeuten (vgl. auch Aguado 2004: 30), ist sie selbst bei relativ fließenden L2-Sprechern nicht durchgängig anzunehmen. Bei den im Folgenden ausschnittsweise dargestellten Daten Lauten Denkens war in einem vorgeschalteten Training explizit darauf hingewiesen worden, dass die Versuchspersonen jederzeit zwischen Englisch (L2) und Deutsch (L1) wechseln können. Wie die folgenden Ausschnitte aus Lautdenkprotokollen exemplarisch zeigen, vollziehen sich derartige Wechsel jedoch nicht reibungslos und unterbleiben häufig ganz, selbst wenn Formulierungsnot in der L2 auftritt.

Datenbeispiel 1

... turn the rem- . remaining forest into into conservation areas . . or national parks $(1 \mathrm{~s})$ so . nobody $(1 \mathrm{~s})$ can . ääh (1 s) can . [(betont) do] some- äh can destruct it. . . There . is (1 s) ääh there are (2 s) if they don’t . ääh (1 s) äähm äähm [(d) abholzen] it (1 s) äh. . the- . . if they are national parks they can't destruct it.

Datenbeispiel 2

but $(2 \mathrm{~s})$ I think . . that's not the the whole $(1 \mathrm{~s})$ eer . because $(1 \mathrm{~s})$ the people . . [(betont) need] . . ähmmm (1 s) äh . . wood . and and and and (1 s) and äh (1 s) things like that (1 s) so (1 s) they have to ähm (1 s) take it somewhere.

Aus den Protokollen lassen sich lexikalische Suchprozesse inferieren, die von Häsitationsphänomenen und Pausen begleitet werden. In Datenbeispiel 1 sehen wir, dass erst nach einem längeren Suchprozess der Wechsel in die L1 vollzogen wird; in Datenbeispiel 2 wird die Lexiklücke in der L2 nach Suchprozessen durch eine Paraphrasierung aufgelöst.

Bei dieser Probandin lässt sich somit nicht annehmen, dass das oben dargestellte Modell die unterliegende kognitive Struktur adäquat abbildet. Vielmehr scheint die einmal gewählte Sprache einen Sprachmodus (Grosjean 1997) zu aktivieren, den Sprecher nicht ohne weiteres wieder verlassen (vgl. auch 
Heine 2005), was bei Formulierungsnot in der L2 separate Problemlöseschritte auslöst (Abbildung 3). Diese können verschiedene Resultate mit sich bringen (vgl. Alternativen 1-5 in Abbildung 3).

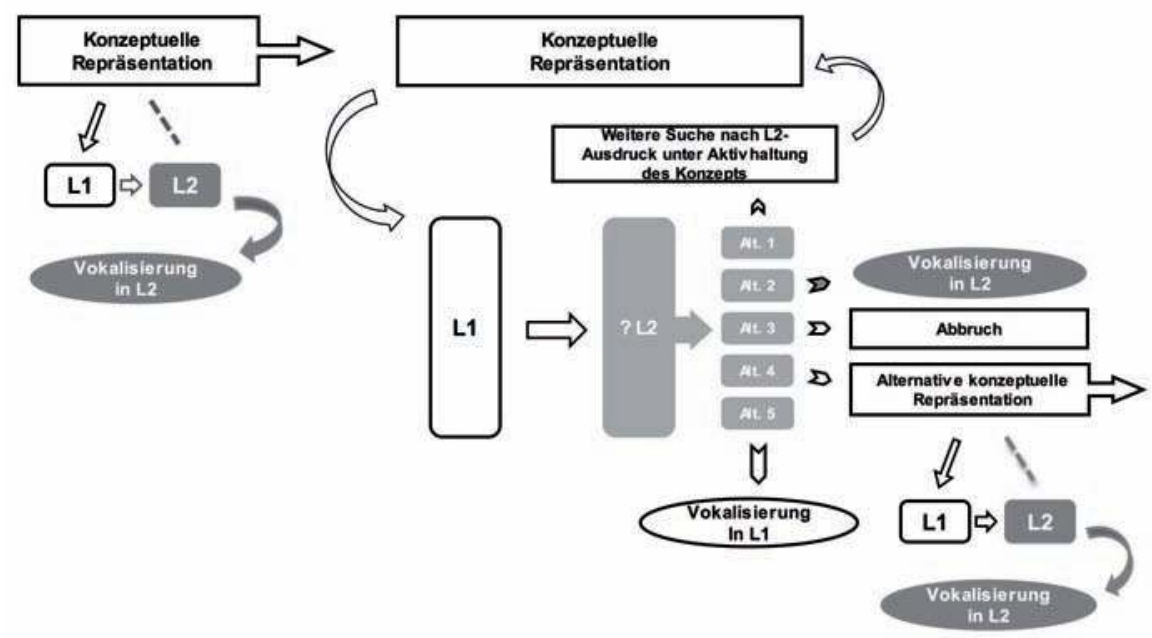

Abbildung 3: Verharren im L2-Sprachmodus führt bei weniger stark mit dem unterliegenden Konzept verknüpften L2-Formen zu separaten Problemlöseschritten in Form von Suchaktivitäten für die Formulierung in der L2. Dies kann unterschiedliche Lösungsalternativen bedingen, die aber alle die unterliegende konzeptuelle Struktur verändern.

Hat eine L1-dominante Versuchsperson, die sich im L2-Modus befindet, eine Vorstellung davon, was sie zum Ausdruck bringen will und kann dafür aber nicht unmittelbar eine entsprechende L2-Form aktivieren, so führt dies als eine Möglichkeit dazu, dass ein Suchprozess beginnt (Alternative 1), mit dem Resultat, dass das entsprechende Konzept länger mental aktiv gehalten wird als bei einer automatischen Aktivierung, was u. a. Gedächtnisspuren vertieft. Ein solch vertiefter Suchvorgang kann entweder zu einem Auffinden einer entsprechenden L2-Form mit anschließender Vokalisierung führen (Alternative 2) oder erfolglos bleiben und mit einem Abbruch der ursprünglichen Vokalisierungsabsicht enden (Alternative 3). Eine weitere Möglichkeit besteht darin, dass die Versuchsperson feststellt, dass für den konzeptualisierten Gedanken keine entsprechende L2Form abrufbar ist und daher eine Formulierungsalternative gesucht wird, wie in Datenbeispiel 2 oben (Alternative 4), also versucht wird, den Gedanken anders auszudrücken. Dies kann beispielsweise durch ein Einkreisen des ursprünglichen konzeptuellen Inhalts durch Aktivierung von Hyponymen, Hyperonymen oder Beispielen für den Ursprungsgedanken geschehen. Derartige Vorgänge haben Auswirkungen auf die kognitive Aktivität, denn sie bewirken eine Aktivierung von Sinnzusammenhängen und damit eine Vertiefung von Gedächt- 
nisinhalten bzw. Lernprozesse. Schließlich gibt es daneben noch eine weitere Möglichkeit (Alternative 5), nämlich das Ausweichen auf die Vokalisierung in der L1, wenn keine adäquate L2-Form gefunden werden kann wie in Datenbeispiel 1 oben.

Dieser Sprachmodus führt dazu, dass bei subordinate bilinguals (Weinreich 1970), bei denen die L1 stärker mit der konzeptuellen Repräsentation verknüpft scheint als die L2 und damit die Produktion der L2-Form häufig Suchprozessen unterliegt (wie es in den oben angeführten Datenbeispiele sichtbar wird), die Gedanken selbst verändert werden; so wird beispielsweise länger bei ihnen verweilt, es werden benachbarte Konzepte für Paraphrasierungen aktiviert etc., also Effekte auf die unterliegende kognitive Repräsentation verursacht. Es erscheint nicht plausibel, ähnliche Unterbrechungen und separate sprachliche Suchschleifen auch bei stiller Bearbeitung einer Aufgabe anzunehmen.

Verkompliziert wird die Situation noch dadurch, dass empirisch fundierte neuere Modelle des mehrsprachigen mentalen Lexikons die aus der Sprachkontaktforschung stammende Konzeption von coordinate bilingualism integrieren, indem sie nicht von einer einzigen konzeptuellen Repräsentation ausgehen, die mit den L1- und L2-spezifischen lexikalischen Repräsentationen verknüpft ist, sondern darauf verweisen, dass neben gemeinsamen Konzepten durchaus auch L1- und L2-spezifische Konzepte vorliegen können (s. Pavlenko 2009 für einen Überblick und hier insbesondere Pavlenkos Revised Hierarchical Model). Dies wird möglich, weil zwischen semantischen und konzeptuellen Repräsentationen unterschieden wird, die in traditionellen Modellen des mehrsprachigen mentalen Lexikons zumeist nicht auseinandergehalten werden (s. Diskussion in Grosjean 1998, Paradis 1997, Pavlenko 2009). In unserer Modelldarstellung würde dies wie in Abbildung 4 aussehen. Statt eines einzigen unterliegenden Konzepts haben wir es hier mit leicht verschiedenen Gedankeninhalten zu tun, die an die jeweilige Einzelsprache gebunden sind. Bei Verwendung der L1 werden L1-spezifische Konzepte aktiviert (s. Abb. 4); bei Verwendung der L2 würde dagegen auf davon verschiedene L2-spezifische Konzepte zurückgegriffen (s. Abb. 5).

Hier ist außerdem zu beachten, dass die Pfeile nun nicht mehr nur von der konzeptuellen zur lexikalischen Repräsentation und weiter zur Vokalisierung weisen, sondern ebenso in die umgekehrte Richtung; die jeweilige Sprachwahl ist also einerseits ein Symptom des jeweils gedachten sprachdomänenspezifischen Gedankens, aber eine einmal gewählte Sprache aktiviert auch reziprok die jeweils mit ihr verbundenen Konzepte. Wenn also in einen L1-Vokalisierungsmodus eingetreten wird, werden L1-spezifische Konzepte aktiviert, was wiederum einen Einfluss darauf hat, was gedanklich abläuft. Die Verwendung der L2 aktiviert dagegen entsprechende L2-spezifische Konzepte. Die unterliegende Gedankensequenz ist hier eine andere und die Verbalisierung von kon- 
zeptuellen Inhalten hat damit einen reaktiven Effekt auf die zu untersuchende Gedankentätigkeit.

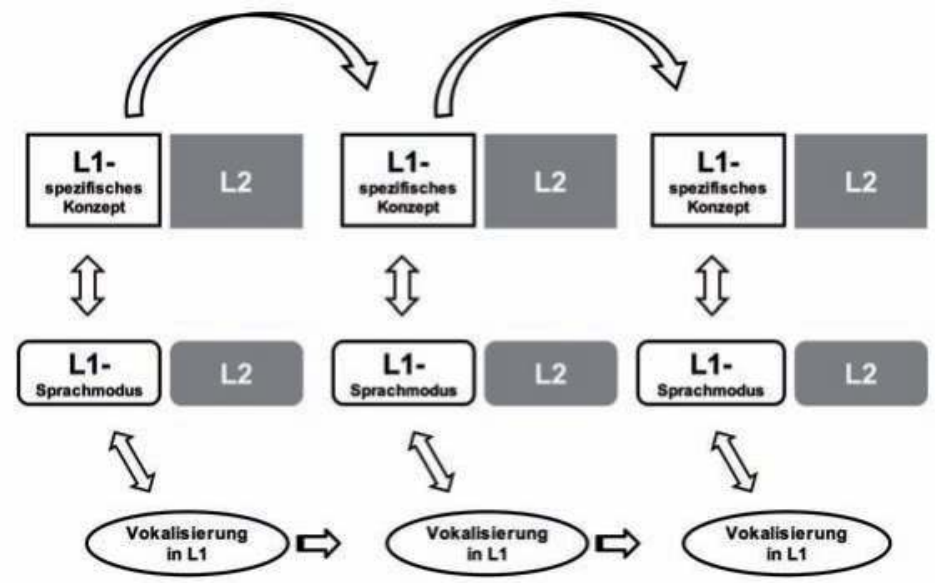

Abbildung 4: Wenn L1- und L2-spezifische Konzepte vorliegen, bedingt ein Sprachmodus auch die Aktivierung dieser Konzepte und damit jeweils nach Denksprache andere gedankliche Inhalte (hier: L1-Sprachmodus bedingt L1-spezifische Konzepte)

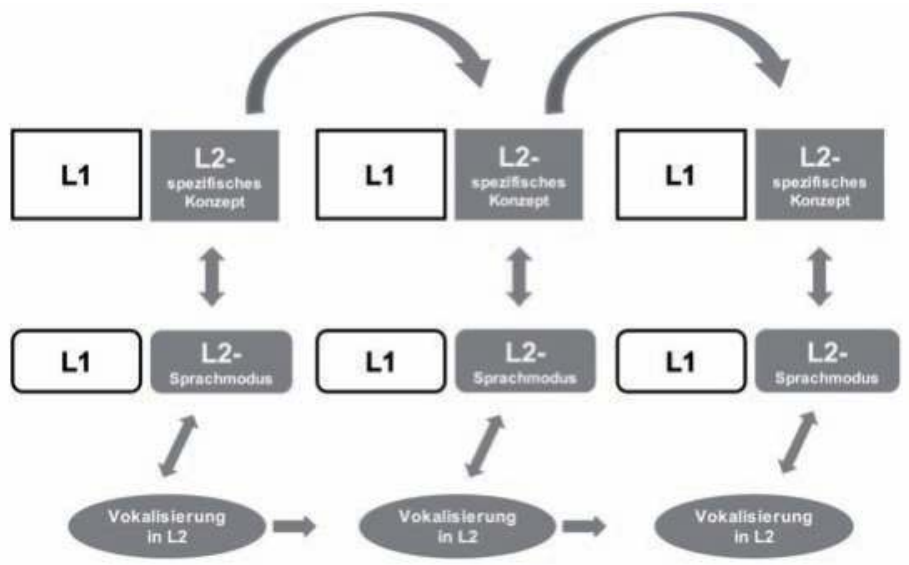

Abbildung 5: L2-Sprachmodus bedingt L2-spezifische Konzepte

Neben den angesprochenen Aspekten ist davon auszugehen, dass Mehrsprachigkeit nicht nur in den Weinreich'schen Formen subordinate, coordinate oder compound als Reinform vorkommt, sondern dass individuell ausgeprägte Mischformen von Repräsentationsformaten anzunehmen sind. Diese könnten beispielsweise bedingt sein durch: a) Kompetenzniveau in den unterschiedlichen 
Fertigkeiten; wenn eine Person zwar eine hohe schriftsprachliche Kompetenz in der L2 aufweist, muss dies nicht bedeuten, dass sie flüssig und automatisiert in der L2 verbalisieren kann; b) individuelle domänenspezifische Kompetenz; möglicherweise kann eine Person relativ unbehindert in bestimmten thematischen Feldern die L2 benutzen, in anderen jedoch ungeübt in ihrer Verwendung sein, was zu lexikalischen Suchaktivitäten führt; c) Lern- bzw. Erwerbskontext der L2; wurde die L2 in kulturell andersartigen Kontexten erlernt als die L1, sind möglicherweise auch andersartige Konzepte mit dem jeweiligen Lexikon verbunden. Es ließe sich eine Vielzahl weiterer Punkte anführen - u. a. die Anzahl der verfügbaren Fremdsprachen, Ähnlichkeitsgrad der beherrschten Sprachen, die bereits angesprochene Verbosität der Versuchsteilnehmerinnen -, die darüber hinaus auch noch über die Zeit Veränderungen unterworfen sein können (e.g. De Groot 1993, Herdina/Jessner 2002, Pavlenko 2009). Eigentlich müsste man also beim Einsatz von Lautem Denken bei mehrsprachigen Sprechern diese Fülle an Variablen kontrollieren, um valide Daten zu erhalten.

Aus diesen Überlegungen sollte deutlich geworden sein, dass die Frage danach, ob Lautes Denken in der Fremdsprache ohne Artefakte der Datenerhebungsmethode möglich ist oder nicht, pauschal nicht beantwortet werden kann. Ericsson/Simon (1993) gehen hier von einer vereinfachten Sicht aus, die als mögliche Auswirkung der Verwendung der L2 lediglich eine Verlangsamung der Prozesse, aber sonst keine Auswirkungen auf die unterliegenden kognitiven Prozesse voraussagt:

Speech in a foreign language illustrates how encoding processes that are not automatic slow processes down. Persons fluent in a second language can usually think aloud in that language even while thinking internally in the oral code of their native language or in non-oral code. In this case, there is nearly a one-to-one mapping between structures in the oral code of the first language and the code of the second language that is used for vocalization. How much the thinking is slowed down will then be a function of the subject's skill in the second language. (Ericsson/Simon 1993: 249ff.)

Lediglich eine Verlangsamung ist, wie oben ausgeführt, jedoch nur dann anzunehmen, wenn wir es mit höchst verbalen, wirklich ausgeglichenen Mehrsprachigen zu tun haben, was in der Realität selten der Fall sein dürfte.

Für die Erhebung von Lautdenkdaten bedeutet dies, dass Forschende sich zumindest darüber bewusst sein müssen, dass bestimmte Artefakte, die nicht unmittelbar kontrollierbar sind, in den Daten auftauchen werden. Diese zu identifizieren und einzuschätzen muss Teil der Dateninterpretation sein. Eine detaillierte Ausarbeitung der angesprochenen Aspekte in Bezug auf introspektive Verfahren steht hier allerdings - auch international - noch aus. 


\section{Abschließende Bemerkungen}

Ziel dieses Beitrags war es, den Stand der Forschung zu introspektiven Verfahren kurz zu umreißen, neuere Entwicklungen aufzuzeigen sowie Desiderata $\mathrm{zu}$ formulieren. Aus den obigen Ausführungen sollte deutlich geworden sein, dass es sich bei den Desiderata insbesondere darum handelt, das Spezifikum der Mehrsprachigkeit und damit Aspekte der mehrsprachigen Sprachverarbeitung für den Einsatz introspektiver Verfahren genauer zu erfassen, was sich nur durch eine kritische Auseinandersetzung mit Modellen des mehrsprachigen mentalen Lexikons und psycholinguistischen Erkenntnissen zur mehrsprachigen Sprachproduktion wird realisieren lassen. Daneben sind die Bereiche, die hier als neuere Entwicklungen bezeichnet wurden, zwar bereits ansatzweise diskutiert worden, jedoch ergibt sich auch hier noch weiterer Forschungsbedarf. Dies bezieht sich vor allem auf die Fragen, inwiefern introspektive Verfahren in einem soziokulturellen Theorierahmen zu bewerten sind, wie emotionale, situationale und soziale Aspekte in introspektiven Daten ausmachbar und auszuwerten sind und wie die Einsicht auf die Methodikdiskussion übertragen werden kann, dass Menschen verschiedenen verbalen Typen angehören; schließlich ist insbesondere für die Fremdsprachenforschung von zentraler Bedeutung, dass Mehrsprachigkeit mehrdimensional, dynamisch und in jeweils individueller Ausprägung vorzustellen ist und sich daraus gewichtige Konsequenzen für den Einsatz von Lautem Denken ergeben. In diesen Bereichen wären weitere empirisch gestützte Untersuchungen von Wert, die kritisch diskutieren, wo die Grenzen und Möglichkeiten introspektiver Verfahren liegen.

\section{Literaturhinweise}

Aguado, Karin (2004). Introspektive Verfahren in der empirischen Fremdsprachenerwerbsforschung. Methodisch-methodologische Überlegungen. Fremdsprachen und Hochschule: 24-38.

Arras, Ulrike (2007). Wie beurteilen wir Leistung in der Fremdsprache? Strategien und Prozesse bei der Beurteilung schriftlicher Leistungen in der Fremdsprache am Beispiel der Prüfung Test Deutsch als Fremdsprache (TestDaF). Tübingen: Narr.

Arras, Ulrike (2009). What's on a rater's mind? Die Erforschung von Beurteilungsstrategien und ihre Bewusstmachung durch Schulungsmaßnahmen als Voraussetzungen für die Testvalidität. ZfAL 50: 33-45.

Beermann, Christian / Cronjäger, Hanna (2011). Freude-, Langeweile- und Angsterleben im Verlauf der Sekundarstufe I im Fach Französisch: Welche Rolle spielt die Wertschätzung des Faches? In: Göbel, Kerstin / Hartig Johannes / Rauch Dominique (Hrsg.): Schwerpunktausgabe ,Empirische Methoden und Ergebnisse in der Fremdsprachenforschung' der Zeitschrift für interkulturellen Fremdsprachenunterricht. Online: zif.spz.tu-darmstadt.de/ jg-16-2/beitrag/Beermann_Cronjaeger.pdf (letzter Aufruf [17.09.12]). 
Behrend, Jana (in Vorbereitung). Fremdsprachenlernen im Kontext von rezeptiver Mehrsprachigkeit. Dissertation, Technische Universität Darmstadt.

Beyer, Sabine (2005). Introspektive Verfahren im fremdsprachlichen Unterricht. Deutsch als Fremdsprache 42/1: 18-22.

Börner, Wolfgang / Vogel, Klaus (2004) (Hrsg.). Emotion und Kognition im Fremdsprachenunterricht. Tübingen: Narr.

Bowles, Melissa (2010): The think-aloud controversy in second language research. New York: Routledge.

Dahnken, Astrid (2005). Englisch in der Hauptschule: Eine didaktische Rekonstruktion von fremdsprachlichem Unterricht und bilingualem Unterricht. Oldenburg: BIS-Verlag.

De Groot, Annette (1993). Word-type effects in bilingual processing tasks: Support for a mixed-representational system. In: Schreuder, Robert / Weltens, Bert (eds.): The bilingual lexicon. Amsterdam: John Benjamins. 27-51.

De Groot, Annette / Poot, Rik (1997). Word translation at three levels of proficiency in a second language: The ubiquitous involvement of conceptual memory. Language Learning 47: 215-264.

Diao, Lan (2010). Strategien beim Lesen chinesischer Texte. Ermittlung der Lesestrategien deutscher Schülerinnen und Schüler durch Lautes Denken. Chinesischunterricht 25: 172185 .

Eckerth, Johannes (2003). Fremdsprachenerwerb in aufgabenbasierten Interaktionen. Tübingen: Narr.

Ericsson, K. Anders / Simon, Herbert A. (1993). Protocol analysis: Verbal reports as data. Cambridge, Mass: MIT Press. 2., überarb. Aufl.

Ericsson, K. Anders / Simon, Herbert A. (1987). Verbal Reports on Thinking. In: Færch, Claus / Kasper, Gabriele (eds.): Introspection in second language research. Clevedon: Multilingual Matters. 24-53.

Feick, Diana (in Vorbereitung). Autonomes Lernen als Sozialer Prozess. Autonomiepotenziale gruppeninterner Aushandlungsprozesse am Beispiel von Handy-Videoprojekten im DaFUnterricht. Dissertation, Universität Leipzig.

Gass, Susan M. / Mackey, Alison (2000). Stimulated recall methodology in second language research. Mahwah, NJ: Erlbaum.

Göpferich, Susanne (2006). Popularization from a cognitive perspective - what thinking aloud and log files reveal about optimizing reverbalization processes. Fachsprachel International Journal of LSP 3-4: 128-154.

Grosjean, Francois (1997). Processing Mixed Language: Issues, Findings, and Models. In: de Groot, Annette / Kroll, Judith (eds.): Tutorials in Bilingualism: Psycholinguistic Perspectives. Mahwah, NJ: Erlbaum. 225-254.

Heine, Lena (2005). Lautes Denken als Forschungsinstrument in der Fremdsprachenforschung. Zeitschrift für Fremdsprachenforschung 16/2: 163-185.

Heine, Lena (2010). Problem solving in a foreign language. Berlin, etc.: Mouton de Gruyter. 
Heine, Lena (erscheint). Introspektion. In: Settinieri, Julia / Demirkaya, Sevilen, Feldmeier, Alexis / Gültekin-Karakoc, Nazan / Immich, Stephanie / Riemer, Claudia (Hrsg.): Einführung in empirische Forschungsmethoden für Deutsch als Fremd- und Zweitsprache. UTB.

Heine, Lena / Schramm, Karen (2007). Lautes Denken in der Fremdsprachenforschung: Eine Handreichung für die empirische Praxis. In: Vollmer, Helmut Johannes (Hrsg.): Synergieeffekte in der Fremdsprachenforschung. Frankfurt am Main: Lang. 167-206.

Herdina, Philip / Jessner, Ulrike (2002). A dynamic model of multilingualism. Perspectives of change in psycholinguistics. Clevedon, etc.: Multilingual Matters.

Ishitsuka, Izumi (in Vorbereitung). Lernerstrategien und Lernprozesse japanischer Deutschlerner in der zielsprachlichen Kommunikation im Kleingruppenunterricht - eine qualitative Untersuchung. Dissertation, Universität Kassel.

Knorr, Petra / Schramm, Karen (2012). Datenerhebung durch Lautes Denken und Lautes Erinnern in der fremdsprachendidaktischen Empirie. In: Doff, Sabine (Hrsg.): Fremdsprachenunterricht empirisch erforschen: Grundlagen, Methoden, Anwendung. Tübingen: Narr. 184-201.

Knorr, Petra (in Vorbereitung). Kooperative Unterrichtsplanungsgespräche angehender Englischlehrender. Dissertation, Universität Leipzig.

Lindemann, Beate (2009). Lautes Denken und retrospektive Betrachtungen beim Übersetzen aus dem Norwegischen ins Deutsche. Beeinflusst die L2 Englisch das Übersetzen aus der L1 Norwegisch in die L3 Deutsch? In: Baumann, Beate / Hoffmann, Sabine / Nied Curcio, Marina (Hrsg.): Qualitative Forschung in Deutsch als Fremdsprache. Frankfurt a.M. etc.: Peter Lang. 23-44.

Matias, Julio (in Vorbereitung). Wissenschaftliches Schreiben in der Fremdsprache Deutsch: Eine empirische Untersuchung. Dissertation, Universität Leipzig

Morkötter, Steffi (2005). Language Awareness und Mehrsprachigkeit. Eine Studie zu Sprachbewusstheit und Mehrsprachigkeit aus der Sicht von Fremdsprachenlernern und Fremdsprachenlehrern. Frankfurt a.M.: Lang

Ogasa, Nicole (2011). Gefühle und Lernen im Fremdsprachenunterricht. Der Einfluss von Gefühlen auf das Lernen. Frankfurt a.M.: Peter Lang.

Paradis, Michel (1997). The cognitive neuropsychology of bilingualism. In: de Groot, Annette and Kroll, Judith (eds.): Tutorials in bilingualism: Psycholinguistic perspectives. Mahwah, NJ: Erlbaum. 331-354.

Pavlenko, Aneta (2005). Emotions and multilingualism. Cambridge: Cambridge University Press.

Pavlenko, Aneta (ed.) (2006): Bilingual minds: Emotional experience, expression, and representation. Clevedon, UK: Multilingual Matters.

Pavlenko, Aneta (2009). Conceptual representation in the bilingual lexicon and second language vocabulary learning. In: Pavlenko, Aneta (ed.): The bilingual mental lexicon: Interdisciplinary approaches. Clevedon, UK: Multilingual Matters. 125-160. 
Raith, Thomas (2011). Kompetenzen für aufgabenorientiertes Fremdsprachenunterrichten. Eine qualitative Untersuchung zur Ausbildung von Fremdsprachenlehrkräften. Tübingen: Narr.

Reinhold, Beate (2011). Prozessvalidierung von Schreibtests. Eine explorative Studie. Deutsch als Fremdsprache 3: 147-156.

Rossa, Henning (2012). Mentale Prozesse beim Hörverstehen in der Fremdsprache. Eine Studie zur Validität der Messung sprachlicher Kompetenzen. Frankfurt a.M.: Peter Lang.

Sasaki, Tomomi (2008). Concurrent think-aloud protocol as a socially situated construct. International Review of Applied Linguistics in Language Teaching 46/4: 349-374.

Schnell, Anna Katharina (in Vorbereitung). Untersuchung zur Entwicklung des fremdsprachlichen Schreibverhaltens von Studierenden. Dissertation, Universität Bremen.

Schramm, Karen (2001). L2-Leser in Aktion. Der fremdsprachliche Leseprozeß als mentales Handeln. Münster etc.: Waxmann.

Shafer, Naomi (2011). Welche Wege führen nach Rom? Zur qualitativen Validierung des Moduls Leseverstehen einer neuen standardisierten B1-Prüfung für Deutsch als Fremdsprache. Unveröffentlichte Masterarbeit, Universität Fribourg.

Weinreich, Uriel (1970). Languages in Contact. Den Hague: Mouton.

Würffel, Nicola (2001). Protokolle Lauten Denkens als Grundlage für die Erforschung von hypertextgeleiteten Lernprozessen im Fremdsprachenunterricht. In: Müller-Hartmann, Andreas / Schocker-v. Ditfurth, Marita (Hrsg.): Qualitative Forschung im Bereich Fremdsprachen lehren und lernen. Tübingen: Narr. 163-186.

Würffel, Nicola (2006). Strategiegebrauch bei Aufgabenbearbeitungen in internetgestütztem Selbstlernmaterial. Tübingen: Narr.

Zawadska, Agnieszka (2011). Transfer aus vorgelernten Sprachen als Lernerleichterung im schulischen Unterricht Polnisch als dritte Fremdsprache? Zum (möglichen) Umgang mit sprachlichem Vorwissen. In: Baur, Rupprecht S. / Hufeisen, Britta (Hrsg.): ,Vieles ist sehr ähnlich: Individuelle und gesellschaftliche Mehrsprachigkeit als bildungspolitische Aufgabe. Baltmannsweiler: Schneider Verlag Hohengehren. 7-29. 


\title{
Zur Differenzierung retrospektiver verbaler Daten: Protokolle Lauten Erinnerns erheben, verstehen und analysieren
}

\section{Petra Knorr}

\begin{abstract}
Retrospective verbal reports have become widely used not only in cognitive psychology but also across educational as well as second language research to investigate people's thought processes while carrying out a task or activity. Recall protocols from a study on the process of cooperative lesson planning by student EFL teachers suggest, however, that participants verbalize a variety of different thoughts while carrying out stimulated recall. The collected data reveal that some of the participants' responses do refer to thoughts that occurred during the action whereas other responses refer to hindsight thoughts that were only evoked during the retrospective interview. Examples from the study will be presented to illustrate that retrospective reports provide multi-layered information that help determine not only what people have thought during a task but also what might have led to their thoughts and actions, how these thoughts can be understood by the researcher and what participants think in general or after doing the task. The article discusses the consequences that arise from these findings for a grounded analysis of retrospective verbal data.
\end{abstract}

\section{Einleitung}

Während das simultane Laute Denken bisher in zahlreichen fremdsprachendidaktisch motivierten Studien erfolgreich verwendet und als Datenerhebungsmethode umfassend diskutiert wurde, haben retrospektive Introspektionsverfahren in der Methodendiskussion bislang relativ wenig Beachtung gefunden. Außer einer Monographie von Gass/Mackey (2000) zum stimulated recall-Verfahren findet eine Auseinandersetzung damit meist nur verkürzt im Rahmen der Darstellung von Untersuchungen statt, die mit retrospektiven Verfahren arbeiten.

Der vorliegende Beitrag widmet sich daher der retrospektiven Erhebung verbaler Daten, indem zunächst im Sinne einer terminologischen Präzisierung eine Einordnung innerhalb der introspektiven Verfahren sowie eine Differenzierung unterschiedlicher Arten von Retrospektion erfolgen. Daran anschließend wird die Erhebungsmethode des Lauten Erinnerns, eine Form von stimulated recall, in den Fokus gerückt, da sie durch ihre wenig intervenierende, offen gestaltete Art der Durchführung Daten verspricht, die umfassende Einblicke in handlungsleitende Denkprozesse der ForschungspartnerInnen gewähren. Die Vielschichtigkeit retrospektiver Verbalprotokolle, die mit Lautem Erinnern oder ähnlichen Verfahren im Rahmen von diversen empirischen Studien bislang erhoben wurden, legt jedoch gerade vor dem Hintergrund neuerer soziokultureller Ansätze nahe, dass es unbedingt genauer zu erfassen und forschungsme- 
thodisch zu reflektieren gilt, welche Art von Daten tatsächlich auf diese Weise erhoben werden können.

Daher soll im Anschluss an eine Darstellung der Durchführung von Lautem Erinnern eine Analyse von Daten Lauten Erinnerns erfolgen, die im Rahmen einer Studie zu kooperativen Unterrichtsplanungsprozessen von Studierenden im Kontext der Fremdsprachenlehrerausbildung erhoben wurden (Knorr in Vorbereitung). Die Erinnerungsprotokolle der Teilnehmenden werden daraufhin untersucht, inwiefern tatsächlich erinnerte, handlungsleitende Kognitionen geäußert und welche weiteren Gedanken bzw. Sprechabsichten verbalisiert wurden.

\section{Terminologische und metatheoretische Einbettung}

$\mathrm{Zu}$ den introspektiven Verfahren der Datenerhebung werden vorrangig jene Verfahren gezählt, die mentale Aktivitäten und/oder individuelle Einstellungen, Überzeugungen und Wissensbestände rekonstruieren möchten, indem Personen selbst Auskunft über ihre Kognitionen und Emotionen geben (vgl. Aguado 2004, Beyer 2005, Heine 2005). Hinsichtlich einer Einordnung, welche Erhebungsverfahren als introspektiv bezeichnet werden, herrscht bislang jedoch begriffliche Uneinigkeit.

Folgt man einem eher weiten Verständnis, so können neben simultanen und retrospektiven Verbalisationsverfahren, die einen konkreten Handlungsbezug aufweisen, auch Befragungen wie z. B. Interviews, Fragebögen oder sogar Umfragen zu den introspektiven Verfahren gezählt werden, wenn sie die Innensicht der Teilnehmenden (z. B. deren Einstellungen, Lernstrategien oder Erfahrungen) erfragen (Aguado 2004: 24, Beyer 2005: 18, Heine 2005: 166ff.).

\begin{tabular}{|c|c|c|}
\hline \multicolumn{3}{|c|}{ Introspektive Verfahren der Datenerhebung } \\
\hline \multicolumn{3}{|c|}{ weitesVerständnis } \\
\hline Simultane Verfahren & \multicolumn{2}{|c|}{ Retrospektive Verfahren } \\
\hline \multicolumn{2}{|c|}{ engesVerständnis } & \multirow[b]{2}{*}{$\begin{array}{l}\text { ohne Bezug auf eine } \\
\text { konkrete Handlung }\end{array}$} \\
\hline \multicolumn{2}{|c|}{ mit Bezug auf eine konkrete Handlung } & \\
\hline $\begin{array}{l}\text { simultan zum } \\
\text { Handlungsverlauf }\end{array}$ & $\begin{array}{l}\text { im Anschluss } \\
\text { an eine Handlung }\end{array}$ & \\
\hline Lautes Denken (think-aloud) & $\begin{array}{l}\text { z. B. Lautes Erinnern, } \\
\text { stimulated recall, } \\
\text { retrospektive Befragungen }\end{array}$ & $\begin{array}{l}\text { z. B. Interviews, } \\
\text { Fragebögen, Tagebücher }\end{array}$ \\
\hline
\end{tabular}

Abbildung 1: Übersicht introspektiver Verfahren der Datenerhebung

Ein engeres Verständnis von introspektiven Methoden (z. B. Dörnyei 2007: 147) umfasst hingegen nur Verfahren, die in einem deutlichen Handlungszusam- 
menhang stehen und darauf zielen, Kognitionen einer Person in Bezug auf eine konkrete Handlung zu erheben. Darunter zählen vorrangig das simultane Laute Denken sowie retrospektive Verfahren wie z. B. stimulated recall. Diese etwas engere Sichtweise, die darauf verzichtet, alle Formen von Selbstauskünften unter die Kategorie der introspektiven Verfahren zu subsumieren, erscheint m. E. sinnvoll, da forschungsmethodisch so mehr Trennschärfe erreicht wird.

Da es sich hierbei um die Erhebung von verbalisierten Kognitionen in Bezug auf einen konkreten Handlungsverlauf (z. B. die Bearbeitung einer Aufgabe oder die Lösung eines Problems) handelt, werden die erhobenen Daten auch als verbal reports (Ericsson/Simon 1987, 1993), verbal protocols (Swain 2006), ,Verbalprotokolle‘ oder ,Lautdenkprotokolle‘ (Heine 2005: 168ff.) bezeichnet.

Für retrospektive Verbalisationsverfahren finden sich die Bezeichnungen retrospective reports (Ericsson/Simon 1987, 1993), delayed retrospection (Færch/ Kasper 1987), ,nachträgliches Lautes Denken“(Weidle/Wagner 1994), stimulated recall (Gass/Mackey 2000), ,verzögerte Retrospektion“ (Würffel 2001) oder ,retrospektive Lautdenkprotokolle' (Heine 2005, Heine/Schramm 2007). In der Forschungsliteratur zeigt sich jedoch, dass die Begriffe z. T. unterschiedlich verwendet werden und das zugrundeliegende Verständnis davon, was die Verfahren ausmacht, differiert.

Unterschiede werden vor allem bezüglich der Zielsetzung, mit der die Verfahren eingesetzt werden, sowie der damit verbundenen Art und Weise der Durchführung der Datenerhebung deutlich. In fremdsprachendidaktischen, bildungs- und sozialwissenschaftlichen Studien lassen sich diesbezüglich zwei Arten retrospektiver Introspektionsverfahren ausmachen: Verfahren, in denen Gedanken fokussiert werden, die erst im Anschluss an eine Tätigkeit im Zuge von Reflexionsprozessen über die abgelaufene Handlung evoziert wurden, sowie Verfahren, die mit dem Ziel eingesetzt werden, mentale Prozesse zu rekonstruieren, die während einer Handlung abgelaufen sind.

retrospektive Introspektionsverfahren mit Bezug auf eine konkrete Handlung

Fokussierung auf (gegenwärtige) Gedanken, die nach der Handlung entstehen

(z. B. retrospektive Befragungen)
Fokussierung auf (erinnerte) Gedanken, die während der Handlung entstanden sind (z. B. Lautes Erinnern)

Abbildung 2: Fokussierungen retrospektiver Datenerhebungsverfahren

Die erstere Form der Retrospektion ist dadurch gekennzeichnet, dass die Teilnehmenden aufgefordert werden, ihre Handlung rückblickend zu kommentieren und zu reflektieren. Diese Erhebungsmethode wird z. B. im Kontext der Lehrerausbildung verwendet, indem Lehrende aufgefordert werden, retrospektiv über ihren (videographierten) Unterricht nachzudenken. Dadurch kann die Fähig- 
keit zur Reflexion über die Handlung (reflection-on-action, Schön 1983) untersucht werden (z. B. Raith 2011, Wyss 2008). Zudem bietet es sich an, das Verfahren auch für Lernzwecke zu nutzen, indem z. B. Studierende oder Lehrende nochmals mit ihrer Unterrichtsgestaltung konfrontiert werden, ihr Verhalten dadurch nachträglich überdenken und alternative Handlungsmöglichkeiten entwickeln können (vgl. De Smet et al. 2012, Reitano/Sim 2010).

Diesem sehr weit gefassten Verständnis von stimulated recall, welches das bewusste Anregen von Reflexion im Moment des Betrachtens einer Videoaufnahme mit einschließt, steht eine engere Sichtweise gegenüber, die die nachträgliche Erhebung von Gedanken fokussiert, die eine Handlung begleitet haben (vgl. Gass/Mackey 2000, Knorr/Schramm 2012). Die Aufmerksamkeit richtet sich hier vordergründig auf die erinnerten Kognitionen der ProbandInnen. Sie werden aufgefordert, alles das auszusprechen, was sie während der durchgeführten Tätigkeit gedacht haben. Die datenerhebende Person tritt dabei meist in den Hintergrund und die Forschungsteilnehmenden übernehmen die aktivere Rolle im Prozess der Datenerhebung (z. B. Henderson/Tallman 2006, Mackey et al. 2000).

Das Laute Erinnern als eine Form der retrospektiven Introspektion basiert auf einem solchen engeren Verständnis. Es soll im Folgenden genauer beschrieben und von ähnlichen Verfahren abgegrenzt werden, um anschließend untersuchen zu können, welche Art von Daten durch ein solches Vorgehen zu erwarten sind bzw. tatsächlich erhoben werden können.

\section{$2 \quad$ Lautes Erinnern}

Der Begriff des ,Lauten Erinnerns' wird für eine möglichst wenig gelenkte Form der Retrospektion verwendet. ${ }^{1}$ Lautes Erinnern bezeichnet „die aus dem Langzeitgedächtnis erfolgende nachträgliche, ungefilterte Verbalisierung einer Person von Gedanken während einer (mentalen, interaktionalen oder aktionalen) Handlung“ (Knorr/Schramm 2012). Mit dem Verzicht, jene Prozesse als nachträgliches Lautes Denken zu bezeichnen, soll ein irreführendes Verständnis der Methode vermieden werden. Denn würde im wörtlichen Sinne nachträglich, d. h. nach Ablauf einer Tätigkeit, laut gedacht werden, dann hieße das, dass Gedanken verbalisiert würden, die erst im Nachhinein, d.h. während der Datenerhebung entstehen (s. Abbildung 2, linke Spalte). Einem eher engen Verständnis retrospektiver Introspektionsverfahren folgend (s. Abbildung 2, rechte Spalte) steht hier vielmehr das Erinnern an bereits während einer Handlung abgelaufene Gedanken im Zentrum. Der Begriff ,Lautes Erinnern' wird in Anlehnung an die englische Bezeichnung stimulated recall verwendet, umfasst

\footnotetext{
${ }^{1}$ Für eine ausführliche Darstellung der Methode des Lauten Erinnerns siehe Knorr/Schramm (2012).
} 
jedoch auch nicht-medienunterstützte Verfahren der nachträglichen Verbalisierung von Gedanken. Er unterstreicht den Aspekt des Rückblicks und benennt das Verbalisieren von Erinnerungen anstelle von Gedanken, die sich auf das Hier-und-Jetzt der Datenerhebung beziehen (ebd.).

Das Laute Erinnern wird verwendet, um retrospektiv Einblicke in mentale Aktivitäten der ForschungspartnerInnen während der Durchführung einer Handlung zu erhalten. Dabei kann es sich um Tätigkeiten einer einzelnen Person oder um dyadische oder polyadische Interaktionen handeln. Es wird u. a. dann eingesetzt, wenn das Laute Denken aufgrund von Interaktionsprozessen (Unterricht, Partner- oder Gruppenarbeit) oder länger andauernden Handlungen nicht gegenstandsangemessen bzw. nicht möglich wäre (ebd.).

Um dem Vergessen von Gedanken entgegenzuwirken, wird das Laute Erinnern möglichst direkt im Anschluss an die Handlung bzw. geringfügig zeitverzögert durchgeführt, so dass noch zahlreiche Erinnerungen verfügbar sind, die noch nicht durch weitere Informationen überlagert wurden. Außerdem wird vielfach der Einsatz von Medien empfohlen, der den Erinnerungsprozess unterstützen soll (Dörnyei 2007: 149, Henderson/Tallman 2006: 77, Mackey 2006: 371f.). Dabei kann es sich um Produkte, die während der Handlung entstanden sind (z. B. ein produzierter Text), um Audio- oder um Videodaten handeln. Die Teilnehmenden werden nach der Tätigkeit damit konfrontiert und aufgefordert, sich anhand der medialen Impulse an ihre Gedanken zu erinnern.

Folgt man den Hinweisen, die für eine optimale Durchführung des simultanen Lauten Denkens gegeben werden (vgl. Ericsson/Simon 1987, 1993, Heine/Schramm 2007), ist auch beim Lauten Erinnern eine möglichst wenig strukturierte, offene Form der Datenerhebung zu bevorzugen (vgl. Gass/Mackey 2000). Die ForschungspartnerInnen werden während der Instruktion aufgefordert, alles auszusprechen, was sie während der Handlung gedacht haben und woran sie sich zum Zeitpunkt der Datenerhebung erinnern. Dadurch soll gewährleistet werden, dass die Teilnehmenden möglichst unbeeinflusst die Gelegenheit bekommen, sich an die Gedanken, die sie während einer Tätigkeit hatten, zu erinnern und diese $\mathrm{zu}$ verbalisieren. Bisher ist noch nicht ausreichend empirisch erforscht worden, welchen Einfluss eine Lenkung der Aufmerksamkeit der Teilnehmenden durch gezielte Fragen der datenerhebenden Person hat. Es wird vermutet, dass direktes Fragen nach spezifischen Gedanken, die die Teilnehmenden während der Handlung hatten, das Verbalisieren handlungsrechtfertigender Äußerungen fördert:

Asking subjects to recall thoughts of specified kinds is not recommended, unless the experimenter has previously established that thoughts of such kinds occur with all subjects. Otherwise it might bias subjects towards accepting low-confidence memories or even towards fabricating such thoughts. (Ericsson/Simon 1987: 42) 
Findet das Laute Erinnern videounterstützt statt, so werden die Teilnehmenden angeregt, das Video eigenständig und so oft wie möglich zu unterbrechen, um ihre erinnerten Gedanken zu verbalisieren. Sollte es nötig sein, dass aufgrund sehr seltener selbstinitiierter Unterbrechungen die datenerhebende Person die Aufnahme stoppt, wird versucht, die Verbalisierung durch eine nicht-direktive Frage nach den Gedanken anzuregen, die der/dem Teilnehmenden an dieser Stelle durch den Kopf gegangen sind (Knorr/Schramm 2012). Auch Gass/ Mackeys Hinweise zur Durchführung von stimulated recalls legen eine offene Struktur und eine möglichst geringe Beeinflussung des Erinnerungsprozesses durch die forschende Person nahe (2000: 57ff.).

Ist die zu untersuchende Tätigkeit relativ kurz, kann die gesamte Handlung im Fokus der Retrospektion stehen (kontinuierliches Lautes Erinnern, z. B. Henderson/ Tallman 2006). Ist die Handlung von längerer Dauer, was vor allem bei Interaktionsprozessen mit mehreren Teilnehmenden der Fall ist, wird meist eine Auswahl von Sequenzen getroffen, die für die Retrospektion genutzt werden (selektives Lautes Erinnern, z. B. Feick in Vorbereitung, Knorr in Vorbereitung). Die Auswahl kann aus rein erhebungspraktischen Gründen (Reduzierung der Länge der Impulsdaten) oder mit einem inhaltlichen Fokus erfolgen (Auswahl von Sequenzen, die Daten zur Beantwortung der Forschungsfrage versprechen). Es kann eine Vorauswahl durch die Forscherin/den Forscher erfolgen oder mit den Teilnehmenden gemeinsam während des Lauten Erinnerns entschieden werden, welche Ausschnitte angeschaut bzw. angehört werden.

Eine Datenerhebung durch Lautes Erinnern wird durchgeführt, um retrospektiv Einblicke in mentale Aktivitäten zu erhalten. Vor dem Hintergrund neuerer Paradigmen in Bezug auf menschliche Denkprozesse, auf die Konstruktion von Wissen und auf Lernvorgänge stellt sich jedoch die im Folgenden zu diskutierende Frage, welche Aussagen die auf diese Weise erhobenen Daten tatsächlich zulassen. Bevor die vorliegenden Daten bezüglich dieser Fragestellung untersucht werden, soll das Problem zunächst unter Rückgriff auf kognitionspsychologische sowie soziokulturelle Theorien erörtert werden.

\section{Kognitionspsychologische und soziokulturelle Ansätze}

Die methodische Auseinandersetzung mit simultanen sowie retrospektiven Introspektionsverfahren wird bislang in Anlehnung an Ericsson/Simon (1993) vor allem durch das Informationsverarbeitungsparadigma dominiert, das die Annahme unterstützt, handlungsleitende Gedanken könnten simultan zum Handlungsverlauf verbalisiert werden, solange sie sich im Kurzzeitgedächtnis befinden und dort entsprechende Aufmerksamkeit erfahren (vgl. Færch/Kasper 1987, Gass/ Mackey 2000, Heine 2005, Heine/Schramm 2007). Sollen handlungsrelevante Gedanken nachträglich verbalisiert werden, müssen Informationen, die in das 
Langzeitgedächtnis gelangt sind, zunächst aktiviert, d. h. ins Kurzzeitgedächtnis transferiert werden. Kognitionspsychologischen Informationsverarbeitungsmodellen zufolge wird Kognition als individuelles Phänomen und Sprache als Mittel zum Ausdruck von Gedanken betrachtet.

Seit den 1990er Jahren wird jedoch eine rein kognitionspsychologische Sicht als Grundlage für die Erhebung verbaler introspektiver Daten von einigen VertreterInnen neuerer soziokultureller Ansätze zunehmend in Frage gestellt (Pressley/Afflerbach 1994, Sasaki 2008, Smagorinsky 1998, 2001, Swain 2006). Soziokulturelle Ansätze betonen vor allem die Bedeutung des sozialen Umfelds und die Rolle der Sprache als bedeutungskonstruierendes Element. Denkprozesse und die Erhebung von Verbalprotokollen werden demnach nicht nur als rein kognitive, sondern vielmehr als sozial situierte Aktivitäten betrachtet (Sasaki 2008: 352). Es wird davon ausgegangen, dass Kommunikation - und damit auch der Prozess der Datenerhebung - immer auch ein Rezipientendesign aufweist, selbst wenn dies durch die Abwesenheit einer datenerhebenden Person versucht wird zu vermeiden (Sasaki 2008, vgl. auch Smagorinsky 1998: 166ff.). Gemäß dieser Sicht werden Gedanken durch Sprache beeinflusst und verändert: „Thought is restructured as it is transformed into speech. It is not expressed but completed in the word" (Vygotski 1987: 251). Verbalprotokolle stellen laut dieser Annahme kein direktes Abbild tatsächlich ablaufender bzw. abgelaufener Denkvorgänge dar, da der Prozess der Versprachlichung eine Veränderung der Kognitionen mit sich bringt (Smagorinsky 1998, 2001).

Die soziokulturelle Perspektive stellt den Sinn der Verwendung introspektiver Verbalisationsverfahren nicht in Frage. Sie erweitert jedoch den Blick und lenkt ihn auf den Kontext und die Bedingungen der Datenerhebung, auf die daran beteiligten Personen, deren Beziehung zueinander und die Faktoren, die deren Kommunikation beeinflussen, auf die Funktion der Sprache sowie auf den daraus resultierenden Aussagegehalt der erhobenen Daten.

Gerade im Bereich der retrospektiven Introspektionsverfahren stoßen ausschließlich kognitionspsychologische Ansätze an ihre Grenzen. Denn allein aufgrund der zeitlichen Verzögerung ist eine selbstadressierte Verbalisierung von Gedanken, wie dies beim Lauten Denken angestrebt wird, kaum möglich, da innere Sprache, wie sie von Ericsson/Simon (1993) beschrieben wird, in ihrer z. T. nonverbalen, bruchstückhaften und fragmentarischen Form zu einem späteren Zeitpunkt in dieser Weise nicht erinnerbar bzw. verbalisierbar ist. Werden Forschungsteilnehmende aufgefordert, ihre Gedanken im Nachhinein zu versprachlichen, wird sich der Sprecher/die Sprecherin immer auch an einem Rezipienten orientieren und um Kohärenz bemüht sein (vgl. auch Feick in diesem Band). Dies führt dazu, dass Gedankengänge ausgeführt oder Kognitionen erklärt werden, so dass für Außenstehende überhaupt erst die Möglichkeit entsteht, einen Gedanken zu verstehen: 
As a mediator it [speech, PK] serves as a means of articulation of thought into a socially comprehensible form. The formal features of speech are determined by the participant's internalized cultural practices and the contingencies that determine appropriate genres for the situation. (Smagorinsky 1998: 173)

Die retrospektiven Äußerungen der ForschungspartnerInnen können sich somit auf Gedanken während der Handlung beziehen, sie bilden diese jedoch nicht direkt ab bzw. geben diese nicht vollständig wieder. Daraus ergibt sich die Frage, ob anhand der Äußerungen der Teilnehmenden tatsächlich auf ihre Kognitionen während der Handlung geschlossen werden kann.

Anhand von Daten aus einer empirischen Studie, in der ForschungspartnerInnen zum Lauten Erinnern aufgefordert wurden, soll diese Fragestellung im Folgenden genauer untersucht werden.

\section{$4 \quad$ Protokolle Lauten Erinnerns}

Ausgangspunkt der folgenden Betrachtungen sind Daten, die mittels Lauten Erinnerns in einer Studie zur Untersuchung von Unterrichtsplanungsgesprächen angehender Englischlehrender erhoben wurden (Knorr in Vorbereitung). Der Studie liegt das Erkenntnisinteresse zugrunde, kooperative Planungshandlungen von Studierenden und die während der Gespräche ablaufenden Denk- und Problemlöseprozesse zu untersuchen, um Einblicke in aktivierte Wissensbestände $\mathrm{zu}$ erhalten und das Lern- oder Erkenntnispotential von Planungsgesprächen zu erforschen.

Die Datenerhebung umfasste Gespräche von jeweils zwei Studierenden, die im Rahmen von Tagespraktika eine Englischstunde gemeinsam planten. Die Videoaufnahmen dieser Interaktion dienten im direkten Anschluss an die Planungsgespräche als Impulse für eine triangulative Datenerhebung mittels medienunterstützten selektiven Lauten Erinnerns, das mit jeder teilnehmenden Person einzeln durchgeführt wurde. Die Auswahl der für das Laute Erinnern genutzten Videoaufnahmen erfolgte gemeinsam mit den Teilnehmenden in Hinblick auf eine zeitlich gut zu bewältigende Impulsdatenmenge. Die ForschungspartnerInnen wurden aufgefordert, die Aufnahme zu unterbrechen, wenn sie sich daran erinnerten, was sie während des Gesprächs gedacht hatten. Kam es zu längeren Phasen, in denen die Aufnahme von den Teilnehmenden nicht unterbrochen wurde, stoppte die Forscherin das Video und forderte die Teilnehmenden auf, ihre erinnerten Gedanken auszusprechen.

Erste Analysen des erhobenen Datenmaterials zeigten, dass zahlreiche Äußerungen der ForschungspartnerInnen Rückschlüsse über ihre Gedanken während der Partnerinteraktion zulassen. Des Weiteren konnten Beobachtungen aus anderen Studien bestätigt werden, die feststellten, dass Teilnehmende trotz des oben beschriebenen Vorgehens neben den Gedanken, die die Handlung begleiteten, 
auch Gedanken äußerten, die erst im Moment des Betrachtens entstanden waren (vgl. De Smet et al. 2012, Edwards-Leis 2006, Weidle/Wagner 1994).

In der Forschungsliteratur werden in Bezug auf die Analyse retrospektiver Verbalprotokolle meist wenig konkrete Aussagen gemacht. Oftmals werden z. B. Analyseschritte nicht im Detail expliziert. Eine ausführlichere Darstellung liefern jedoch Henderson/Tallman (2006: 80ff.) in ihrer Studie, in der sie Bibliothekarinnen in Einzelgesprächen mit Schülerinnen und Schülern untersuchten. Sie gehen davon aus, dass grundsätzlich zwei Arten von Kognitionen unterschieden werden können, die während des von ihnen durchgeführten stimulated recall erhoben wurden: (1) recall thoughts (erinnerte Gedanken) und (2) hindsight thoughts (postaktionale Gedanken, die im Nachhinein entstehen). Erinnerte Gedanken sind während der Handlung entstanden, sie werden retrospektiv erinnert und versprachlicht, während postaktionale Gedanken erst nach der Handlung entstehen:

The recall thoughts occurred during the performance of the task, the 'there and then' or retrospective thoughts, whereas the hindsight thoughts occurred during the interview, the 'here and now' or interpretative thoughts. [...] Some of these 'there-and-then' thoughts provide their reasoning and justification for what they were thinking at that particular time in the lesson. We label these in-action thoughts. In contrast, the hindsight thoughts are only now, an hour after the lesson, occurring to the teacher librarians. Although spontaneous, they are reflective as they are hindsight interpretations, explanations, rationalizations, and descriptions of their actions, verbalizations, and nonverbals during the videotaped event. (Henderson/Tallman 2006: 80)

Eine ähnliche Unterscheidung findet sich bei Schepens/Aelterman/van Keer (2007), die Unterrichtsstunden von Studierenden während ihrer Praktika untersucht haben. Sie differenzieren interactive sowie post-active cognitions (Schepens et al. 2007: 463). Auch Weidle/Wagner (1994) führen neben anderen Verbalisationsbereichen, auf die sich die Äußerungen aus ihren Unterrichtsstudien beziehen, Denkprozesse während der Handlung und Denkprozesse während der Beobachtung des Videobandes an (1994: 94f.).

Das vorliegende Datenmaterial soll diesen Überlegungen folgend zunächst daraufhin untersucht werden, inwiefern erinnerte (recall thoughts) und/oder nachträglich evozierte Gedanken (hindsight thoughts) in den Protokollen erkennbar werden, d. h. ob sich die Äußerungen der Teilnehmenden auf aktionale oder auf post-aktionale Kognitionen beziehen. Dabei können die verwendeten Redemittel (,ich dachte“ versus „,ich denke“), der Sinngehalt der Äußerungen und die Handlungen, auf die sich die Erinnerungsprotokolle beziehen (Aussagen, Blicke, Gedanken) genauer betrachtet werden, um Rückschlüsse zu ziehen, welchen zeitlichen Bezug die geäußerten Kognitionen zur Handlung haben. 


\subsection{Erinnerte Gedanken (recall thoughts)}

Folgt man einer soziokulturellen Perspektive, die davon ausgeht, dass aktionale Gedanken (in-action thoughts) durch den Prozess der Versprachlichung nicht genau abgebildet werden können und durch Sprache Veränderung erfahren und Verbalisierungen immer auch ein Rezipientendesign aufweisen, stellt sich die Frage, wie genau und in welcher Form Gedanken erinnert, geäußert und im Kontext der Datenauswertung für Dritte ersichtlich werden. Im folgenden Ausschnitt aus einem Unterrichtsplanungsgespräch zwischen den Teilnehmerinnen Anja (A) und Heike $(\mathrm{H})^{2}$ sowie dem retrospektiven Erinnerungsprotokoll von Heike $\left(\mathrm{H}_{\mathrm{LE}}\right)$ lässt sich der Prozess der Erinnerung und Versprachlichung eines Gedanken nachvollziehen:

$670 \mathrm{~A} \quad$ Was ich machen kann... Nee, weißte, was ich mache? Ich ähm $\bullet$, ich tu das auf ein großes Blatt schreiben, und dass ich das nur mit Magneten dran machen muss.

$671 \mathrm{H} \quad \mathrm{Hm}$ ((nachdenklich)) •... Jà. [An der Tafel...]

[Dass ich es nicht schreibe,] aber dass es trotzdem größer ist als auf ner Folie.

$673 \mathrm{H}$ Jà.

$674 \mathrm{~A} \quad$ So. ((schreibt, 1s)) Also ich brauch also nur das bis sechs anschreiben. • Und dann ((schreibt, 2s)): „Phrasen • auf • großes Blatt ((2s)) anpinnen“ ((schaut zu H, 0,5s)).

$675 \mathrm{H}_{\mathrm{LE}} \quad<$ Ähm, auf Blätter schreiben, die Phrasen, und dann an die Tafel, fand ich gut, die Idee. Und in dem Moment dachte ich mir: „Hoffentlich geht das logistisch!", weil bei mir war das so: Ich hatte das auch, und ich hatte dann A4-Blätter und die ganze Tafel war voll und ich hatte aber noch fünf A4Blätter, die dran mussten, also Tafel vollkommen überschätzt von der Größe her. Und da dacht ich mir... Dann hab ich aber weiter nix zu gesagt, weil es sind ja nur acht Phrasen und das passt definitiv dann hin.>

676 H ((nickt)) Genau. Jà. (PG8)

Den Gedankengang von Heike könnte man sich so vorstellen: Als Anja ihre Idee formuliert, Sätze bzw. Satzteile auf Wortkarten zu schreiben, um diese an die Tafel heften zu können, erinnert sich Heike an die Stunde, die sie selbst zuvor gehalten hat. Vermutlich hat sie bildlich vor Augen, wie der Platz an der Tafel für ihre Wortkarten nicht ausreichte. Die LE-Daten deuten darauf hin, dass sie sich auch an das unangenehme Gefühl erinnert, in der Stunde ad hoc ihren Plan ändern zu müssen, und daran, dass sie bei ihrer Planung etwas nicht richtig berücksichtigt hatte (,also Tafel vollkommen überschätzt von der Größe her“).

\footnotetext{
${ }^{2}$ Die Namen der ForschungspartnerInnen wurden durch Pseudonyme ersetzt. Die Daten des Lauten Erinnerns werden eingerückt, kursiv sowie durch Pfeile eingeschlossen dargestellt. Zur Hervorhebung der Daten des Lauten Erinnerns werden die entsprechenden Passagen eingerahmt. Die Transkription erfolgte in Anlehnung an HIAT (s. Rehbein et al. 2004).
} 
Sie würde Anja dieses Gefühl gern ersparen, überlegt, um wie viele Wortkarten es sich in dieser Stunde handeln würde und kommt zu dem Schluss, dass acht Karten auf der Tafel ausreichend Platz haben werden. Daraufhin beschließt sie, nichts einzuwenden und ihr zuzustimmen. Dieser Gedankengang findet innerhalb kürzester Zeit statt, wahrscheinlich, als sie zögernd „ $\mathrm{Hm}^{\prime \prime}$ sagt und eine kurze Pause entsteht. Ihr entschlossenes ,Jà.“ in Abschnitt 671 deutet darauf hin, dass der Gedankengang bereits beendet ist.

An diesem Beispiel wird deutlich, dass die Teilnehmerin sich an ihre Gedanken zu einem bestimmten Zeitpunkt in einer Weise erinnert, die vermutlich sowohl sprachlich als auch bildlich, emotional und episodisch ist. Um diesen komplexen Gedankengang ausdrücken zu können, muss er in Sprache übersetzt werden. Dadurch erhalten wir ihre Sicht des Gedankens, nicht den Gedanken selbst (vgl. auch Huber/Mandl 1994: 20). Die Orientierung während der Erhebungssituation auf einen Rezipienten führt dazu, dass sie keine Gedankenfragmente oder Bilder verbalisiert, sondern in kohärenter und zusammengefasster Form über ihre Gedanken berichtet.

Erinnerte Gedanken werden demzufolge nicht nur benannt, sondern in Form von Beschreibungen oder Erklärungen ausgedrückt. Eine genaue Unterscheidung, ob ein Gedanke erinnert oder erst im Nachhinein evoziert oder präzisiert wurde, wird dadurch jedoch erschwert. Letztlich sind diese Berichte auch immer Teil des Hier-und-Jetzt, da Informationen im Moment der Datenerhebung aktiviert und verbalisiert werden. Wesentlich für eine Unterscheidung ist jedoch, dass sich die Äußerungen der Teilnehmenden auf eine Erinnerung beziehen.

Dies wird z. T. direkt formuliert, wie z. B. in der LE-Aussage von Heike im Absatz 675: „Und in dem Moment dachte ich mir“. Die Beschreibung erinnerter Gedanken enthält oftmals Redemittel im Präteritum bzw. Imperfekt wie z. B.: „fand ich gut, die Idee“. Der Hauptgedanke wird häufig wörtlich, d.h. in direkter Rede wiedergegeben, wie im obigen Beispiel im Absatz 675: „Hoffentlich geht das logistisch!“".

Die in dieser Studie erhobenen Daten, die deutlich als erinnerte Gedanken zu erkennen sind, ermöglichen Einblicke in die Denkprozesse der ProbandInnen, die allein durch die Interaktionsdaten nicht zu rekonstruieren gewesen wären. Im Gegensatz zu Handlungen, die allein ausgeführt werden, kann bei Interaktionen davon ausgegangen werden, dass die PartnerInnen viele ihrer Gedanken im Gespräch schon aussprechen, d. h. dass diese Gedanken nachträglich gar nicht mehr geäußert werden müssen. Es bleibt jedoch, wie das oben dargestellte Beispiel zeigt, je nach Verhältnis der GesprächspartnerInnen zueinander immer auch ein Teil der Gedanken unausgesprochen. ${ }^{3}$ Dies wird besonders deutlich bei

\footnotetext{
${ }^{3}$ Vergleicht man die Daten aus der Studie von Henderson/Tallman (2006), die Lehr- und Lerngespräche zwischen ExpertInnen und NovizInnen erforschten, mit den Protokollen aus dieser Untersuchung, so erscheint der Anteil nicht geäußerter Kognitionen in Gesprächen mit
} 
Aussagen, die Unverständnis, Zustimmung oder eine gegenteilige Meinung in Bezug auf das vom Gesprächspartner Gesagte ausdrücken. Im folgenden Beispiel äußert Heike z. B. ihre Gedanken, die sich auf die Versuche von Anja beziehen, sich an Inhalte aus einem Seminar zu Dramapädagogik im Fremdsprachenunterricht zu erinnern und Elemente davon in der Stunde anzuwenden. Aus dieser Aussage und mehreren anderen von Heikes LE-Äußerungen geht hervor, dass sie sich nicht auf ,Experimente' wie diese einlassen möchte. Sie widerspricht Anja im Verlauf des Gesprächs jedoch nur an wenigen Stellen:

$521 \mathrm{H}_{\mathrm{LE}}<$ Ich hab da gar nicht weiter drüber nachgedacht, weil für mich war das nicht relevant in dem Moment. Ich hab gedacht: „Nee, das machen wir nicht. “ $[\ldots]>$ (PG8)

Die Verbalisierungen der Teilnehmenden können auch Aufschluss darüber geben, wann genau ein Gedanke, der u. U. auch schon im Gespräch geäußert wurde, entstanden ist und was ihn evtl. ausgelöst hat:

$176 \mathrm{~A}_{\mathrm{LE}}<$ Das ist mir in dem Moment aufgefallen, wo ich das hier vorgelesen habe. Ich hab ja vorgelesen: „Please open your folders and write down the rest of the phrases in the correct order" und da ist mir in dem Moment eingefallen, dass ich das nicht machen kann, weil während die öffnen, kann ich nicht die Aufgabe ansagen, weil dann keiner zuhört.[...]> (PG8)

Werden Gedanken beschrieben und erklärt, die so oder so ähnlich auch schon im Gespräch zum Ausdruck kamen und aus den Interaktionsdaten hätten rekonstruiert werden können, kann die nochmalige Beschreibung durch die Probandin/den Probanden zu einem besseren Verständnis führen bzw. eine Überprüfung dessen ermöglichen, und somit zur Validierung der Videodaten beitragen:

$352 \mathrm{~A}$ Ich lass die das erst mal vorlesen, dann sag ich... $\cdots$ Ähm $\cdots$. also das lass ich erstmal so, ja? „Two students ähm read out the dialogue“. Und dann füge ich jetzt noch hinzu ,nach vorne kommen“.

$353 \mathrm{H}$ Jà.

354 A ((schreibt, 4s)) So. ((1s)) Und dann kann ich ja sagen:

[,Okay now"]...

$355 \mathrm{H}$ [Warte mal! Du/ ihr habt...] - A Ach so. Das ist ja schon die, die Korrekturphase. Das ist es ja dann.

356 A Hmhm.

$357 \mathrm{H}_{\mathrm{LE}} \quad<J a$, da hab ich mich gefragt: „Wann, wann sichern wir denn das Ergebnis?". Und dann in dem Moment: „Ach so, ja, das ist ja die Ergebnissicherung. "> (PG8)

(leicht) hierarchischen Strukturen größer als in kollaborativen Gesprächskonstellationen, in denen die PartnerInnen stärker gleichberechtigt agieren. 
Neben zusätzlichen Informationen über mentale Handlungen geben die Äußerungen der Teilnehmenden auch Aufschluss über Empfindungen (Unsicherheit, Ratlosigkeit, Sicherheit etc.) während des Gesprächs:

$512 \mathrm{~A}_{\mathrm{LE}}<\mathrm{Ja}$, ich bin mir da total unsicher, ob das jetzt ne gute Idee... Also wir verwerfen das auch wieder, weil das gar nicht geht mit so vielen und weil s vor allem gar nicht um die Aussprache geht, [...]> (PG8)

Das erhobene Datenmaterial weist eine Vielzahl an Äußerungen auf, die auf erinnerte Gedanken oder Gefühle schließen lassen, d. h. die diese nennen, beschreiben oder erklären. Eine Reihe weiterer Aussagen verdeutlicht jedoch, dass während der Datenerhebung zusätzliche Gedanken evoziert wurden, die sich stärker im Hier-und-Jetzt verorten lassen. Im Folgenden sollen entsprechend der verschiedenen Inhalte und Funktionen der Äußerungen unterschiedliche Verbalisationsbereiche differenziert werden.

\subsection{Postaktionale Gedanken (hindsight thoughts)}

Die erhobenen Daten zeigen deutlich, dass es neben erinnerten Gedanken auch zu Äußerungen der Teilnehmenden kommt, die auf postaktionalen Kognitionen basieren. Spontane Gedanken während des Betrachtens der Handlung auf Video werden zunächst im Kurzzeitgedächtnis verarbeitet, während im Nachhinein vielfach auch auf Informationen zurückgegriffen wird, die auf frühere Erfahrungen der ForschungspartnerInnen zurückgehen und demnach im Langzeitgedächtnis gespeichert sind:

These hindsight thoughts necessarily retrieve information from long-term memory as it stores the mental models of past actions, thoughts, and ideas that are drawn upon to argue, explain, clarify, justify, and describe their actions and thoughts during the event. (Henderson/Tallman 2006: 80)

Mit den Äußerungen, denen postaktionale Gedanken zugrunde liegen, sind verschiedene Sprechabsichten verbunden. Die Analyse des vorliegenden Datenmaterials unterstützt eine Einteilung in Aussagen, die einen reflektierenden Charakter haben (5.2.1), die Einblicke in Wissensbestände und subjektive Einstellungen geben (5.2.2) und die vorrangig kontextualisierend sind und zum besseren Verständnis der Gesprächsdaten durch Dritte beitragen sollen (5.2.3). Wesentliche Verbalisierungsformen, die sich innerhalb dieser Bereiche am Datenmaterial festmachen lassen, sollen im Folgenden anhand von Beispielen vorgestellt werden. 


\subsubsection{Reflektierende Äußerungen}

\section{Reflexionen bezüglich der Gesprächsinhalte}

Reflexionen entstehen nach der Handlung vor allem durch die nochmalige Konfrontation mit dem eigenen Verhalten in Form einer Videoaufnahme:

\section{$246 \mathrm{H}_{\mathrm{LE}} \quad<$ [...] Obwohl mir jetzt im Nachhinein fünf Minuten auch $n$ bisschen viel erscheint, weil das wirklich einfache Sätze waren und die Vokabeln sind denen bekannt. [...].> (PG8)}

Heikes Äußerung bezieht sich auf eine längere Gesprächssequenz, in der beide darüber sprechen, wie viel Zeit Anja für die Semantisierung von acht Wortschatzelementen, die den Lernenden zum Teil schon bekannt sind, benötigen wird. Anja schätzt die Dauer zuerst auf zehn Minuten ein und Heike korrigiert, dass sie nur fünf Minuten, d. h. deutlich weniger Zeit einplanen würde. Während Heike den Gesprächsverlauf durch das Betrachten der Videoaufnahme noch einmal nachvollzieht, denkt sie in diesem Moment, dass ihr selbst fünf Minuten noch relativ lang vorkommen. Deutlich erkennbar ist der postaktionale Charakter der Äußerung am Gebrauch der Präsensform sowie der Formulierung ,jetzt im Nachhinein“. Die Reflexion von bestimmten Gesprächsinhalten (wie z. B. der Zeiteinteilung) wird durch die Tatsache begünstigt, dass es sich um die Planung von Unterricht handelt, die mit dem Gespräch noch nicht abgeschlossen ist. Die Datenerhebung fand immer vor der Durchführung der jeweiligen Unterrichtsstunde statt und man kann davon ausgehen, dass die Studierenden bis kurz vor der Stunde über ihre Planung nachgedacht haben.

\section{Selbstreflexive Äußerungen}

Neben der Reflexion der Inhalte der gemeinsamen Stundenplanung werden durch das Betrachten der Planungsgespräche auch selbstreflexive Prozesse angeregt und zum Ausdruck gebracht:

$448 \mathrm{H}_{\mathrm{LE}} \quad<$ Fand ich gut, die Idee. Find ich immer gut, wenn das jemand kann. Ich kann das leider nicht, aber ich fand das als Schüler unwahrscheinlich interessant, wenn der Lehrer so $\bullet$ wie in so ne Rolle reingeschlüpft ist, also das fetzt unwahrscheinlich. Aber ich kann... Ich hoffe, dass ich das irgendwann auch mal hinkriege, [...].> (PG8)

Die Studentin begründet in diesem Beispiel ihre Zustimmung zu Anjas Vorschlag, die Dialogsituation mit einer Person spielerisch darzustellen. Sie verbalisiert, dass sie den Vorschlag gut findet und reflektiert in diesem Moment, dass sie ein solches Vorgehen für sich selbst jedoch als schwierig erachtet. Sprachlich wird dies am Gebrauch der Ich-Form deutlich. Sie verwendet keine allgemeine Formulierung, sondern bezieht sich direkt auf sich selbst: „Ich kann das leider nicht“. 


\section{Reflexion des Gesprächsverlaufs}

Es kommt während der retrospektiven Datenerhebung auch zu Äußerungen, die ein Nachdenken über den Gesprächsverlauf signalisieren. Dabei werden z. B. die Rollen der Teilnehmenden, das Gesprächs- oder Planungsverhalten der Partnerin/des Partners, der Einfluss der anderen Person oder die jeweilige Zusammenarbeit reflektiert:

$925 \mathrm{~A}_{\mathrm{LE}}<$ Naja, dann hab ich gedacht: „Naja gut, dann machen wir das eben“. Also wahrscheinlich wenn ich alleine gewesen wäre, hätt ich das nicht gemacht, da hätt ich dann den anderen Text gemacht. Weiß nicht.> (PG8)

\subsubsection{Handlungsübergreifende Gedanken (Wissen, Einstellungen)}

Das Beschreiben von Gedanken, die den Teilnehmenden während des Gesprächs durch den Kopf gegangen sind, führt oftmals dazu, dass die Gedanken oder Aussagen im Gesprächsverlauf erläutert oder begründet werden. Dabei kommt es zu Äußerungen, in denen Wissensbestände, Einstellungen oder Überzeugungen sichtbar werden, die zum Zweck der Begründung aktiviert wurden und die meist relativ situationsunabhängig sind. Zum Teil erwachsen diese Überzeugungen aus den Erfahrungen der Teilnehmenden im Rahmen der Tagespraktika, wie im folgenden Beispiel deutlich wird:

$594 \mathrm{H}_{\mathrm{LE}} \quad<$, ,What does it mean?“ und „What do you think it means?“ ähm in dem Moment hab ich gedacht: • „,̈̈hm wie sagt man das jetzt am besten?“, weil manches... In der Fünften ist es ziemlich schwierig, weil die können einiges, aber - - nicht alles. Und wie formuliert man das am besten? Also Aufgabenstellung formulieren und irgendwas auf Englisch erklären ist immer sehr schwierig gewesen und die Lehrerin, die dort am R.Gymnasium ist, die neigt dann dazu, schon viel Deutsch zu reden. Hat sie auch selber gesagt, aber ich, und Anja auch, also wir sind dann eher der Meinung: „Nee, wir probieren das erst mal auf Englisch und dann kann man immer noch gucken ob sie s verstanden haben". Aber Aufgabenstellungen ist immer auch so ne schwierige Sache: „Verstehen die das jetzt?“ Und deswegen ist dann wiederum im Kopf dann immer Rückkoppeln angesagt, also dass man dann wirklich weiß: „Okay, haben sie s jetzt verstanden oder haben sie s jetzt nicht verstanden." $>$ (PG8)

Zunächst erläutert Heike ihren Gedanken, dass sie in jenem Moment darüber nachdenkt, wie die Frage an die Lernenden am besten zu formulieren sei. Sie erinnert sich dabei wahrscheinlich an ihre Erfahrungen mit der 5. Klasse und daran, dass es schwer einzuschätzen ist, was die Kinder in der Zielsprache schon verstehen. In den ersten vier Zeilen werden folglich erinnerte Gedanken verbalisiert. Die sich daran anschließenden Äußerungen in Bezug auf die Schwierigkeiten der Studierenden bei der Formulierung von Aufgaben, ihre Erfahrung und Meinung hinsichtlich der Verwendung der Zielsprache und ihre Auffassung zum „Rückkoppeln“ scheinen genereller Art zu sein, worauf die Formulierungen „wir 
sind dann eher der Meinung“ oder ,ist immer sehr schwierig gewesen“ hindeuten. Aussagen dieser Art erlauben Einblicke in das sich entwickelnde berufliche Selbstverständnis der Teilnehmenden. So wird z. B. auch in der Erklärung einer Aussage der Teilnehmerin Clara (C) ihre Einstellung in Bezug auf die Vermittlung von Grammatik (in diesem Fall geht es um die indirekte Rede) erkennbar:

219 C Also, ich mein, das ist ne schreckliche Stunde. Das ist ne schreckliche Grammatikstunde, die kann ja nur schrecklich sein ((lacht, 1s)).

220 ILE < Was denken Sie da?>

221 CLE <((lacht, 1s)) Das war auch eher $n$ bisschen ironisch, glaub ich. Also ähm, klar weiß ich, dass man auch Grammatik äh ganz nett machen kann und aktiv vermitteln kann und dass es ja auch wichtig ist und so. Also das ist mir alles schon bewusst, aber meine vorherige Stunde war halt so ne schöne inhaltlich motivierende Stunde einfach nur. Und als ich dann dieses Thema bekommen hab, äh als es dann klar war, dass ich diese Stunde halten muss, da war ich schon ganz schön ähm geschockt und wusste auch gleich, dass es halt nicht so ne schöne Stunde wird, aber... Also, ich find jetzt nicht, dass das ne schreckliche Stunde ist, das ist eher $n$ bisschen ironisch gemeint.> (PG7)

Ein Teil der handlungsübergreifenden Äußerungen verweisen außerdem auch auf generelle Schwierigkeiten oder Problempunkte, die die Studierenden mit der Planung von Unterricht haben:

$216 \mathrm{H} \quad$ Wo sind wir $\mathrm{n}$ jetzt zeitlich?

217 A Keine Ahnung. ((1s)) Müssen wir vielleicht auch mal aufschreiben, ja? ((schaut in ihre Aufzeichnung, 1s)) Ah”, also: „Hello“ bla bla bla „my name is" und so, dauert eine Minute.

218 HLE <Zeit ist immer $n$ riesen Problem bei der Planung. Selbst wenn man die Klasse schon kennt, kann man ganz schlecht einschätzen, wie lange die brauchen. Also das hat auch immer sehr, sehr lange gedauert. Also nicht das zu planen, aber dann am Ende wirklich zu sehen: „Okay, jetzt läuft s ausn Ufern oder nicht". Also das war immer das Problem.> (PG8)

Heikes Gedanken in Bezug auf die Schwierigkeit, die Zeiteinteilung im Unterricht angemessen zu planen, bezieht sich nicht auf eine bestimmte Situation oder Äußerung während des Gesprächs, sondern gilt eher für das gesamte Gespräch oder die gesamte Erfahrung der Teilnehmenden während der Tagespraktika. Die Verwendung von „immer“ und „man“ verweist zusätzlich darauf, dass dieser Aspekt hier als ein generelles Problem wahrgenommen wird.

An bestimmten Stellen im retrospektiven Datenmaterial werden explizit Positionen in Bezug auf die Veränderung und Entwicklung des beruflichen Selbstverständnisses der Teilnehmenden deutlich: 
320 HLE $<$ [...] Und ähm das Problem war die ganze Stunde durchweg, dass nicht alle immer zugehört haben. Und deswegen dacht ich mir: „Okay, das ist genau richtig. Sie sagt, sie öffnen jetzt die Bücher und dann holt sie noch mal den ganzen Fokus von der Klasse auf sich und sagt, was man machen soll. " Also das erachte ich jetzt mittlerweile als ganz wichtig. [...]> (PG8)

In diesem Beispiel wird ein Lernzuwachs oder Erkenntnisgewinn ausgedrückt, der für das Forschungsinteresse der Studie von besonderer Relevanz ist.

\subsubsection{Kontextualisierende Äußerungen}

Hintergrundinformationen für den Rezipienten

Bei der gemeinsamen Betrachtung des videographierten Gesprächsverlaufs werden Momente sichtbar, in denen sich die GesprächspartnerInnen während ihrer Interaktion auf eine gemeinsame Wissensbasis beziehen, die von der Forscherin möglicherweise nicht geteilt wird. Die Erhebungssituation mit einer/einem unwissenden Dritten veranlassen die Teilnehmenden mitunter dazu, die zum Verständnis nötigen Hintergrundinformationen mitzuteilen. Oftmals handelt es sich dabei um die Beschreibung eines vergangenen Ereignisses:

$32 \mathrm{E}$ Und dann kannst du auch noch mal ähm/ auf das Reflexivpronomen eingehen, weil ich das ja einfach mal komplett untern Tisch hab fallen lassen.

33 ELE < ̈̈hm ich hab in meiner Stunde nen Fehler gemacht. Ich hab nur gesagt, das Personalpronomen ändert sich und dann hat Frau T. mich äh drauf hingewiesen, dass sich ja/ dass alle anderen Pronomen sich auch ändern und ähm ich deswegen zu Clara gesagt hab, dass sie darauf noch mal achten soll, dass sie das noch mal deutlich macht, [...].> (PG7)

\section{Erklärung von Nonverbalia und aktionalen Handlungen}

Die Ausrichtung der Verbalisierungen auf einen Rezipienten führt vielfach auch zu Handlungsbeschreibungen. Vor allem Blicke, Gesten oder unverständliche Aussagen, die im Video beobachtet werden und die sich der/dem Forschenden

$A_{L E} \quad<$ Ich suche da... Äh also ich denke da nicht, sondern ich suche ((blättert, 2s)) die Stelle, wo hier drin steht... $\cdots$ Das hier: ((liest, 1s)) ,Ein flexibler Umgang mit den Redemitteln ist erwünscht". Genau. [...].> (PG8)

nicht gleich erschließen würden, werden häufig als Anlass genommen, die Aufnahme zu unterbrechen und die Handlung für eine/n Außenstehende/n zu beschreiben und zu erklären:

$204 A_{L E} \quad<$ Ich suche da... Äh also ich denke da nicht, sondern ich suche ((blättert, 2s)) die Stelle, wo hier drin steht... ・. Das hier: ((liest, 1s)) „Ein flexibler Umgang mit den Redemitteln ist erwünscht". Genau. [...].> (PG8) 


\section{Äußerungen zur Orientierung im Impulsdatenmaterial}

Einige Äußerungen der Teilnehmenden dienen der besseren Orientierung im beobachteten Datenmaterial. Sie zeigen z. B. an, wenn die ForschungspartnerInnen selbst im Moment des Betrachtens überlegen, an welcher Stelle im Gesprächsverlauf man sich befindet, oder wenn sie es für nötig erachten, die datenerhebende Person darüber zu informieren (vgl. auch Weidle/Wagner 1994: 94f.).

$129 \mathrm{~A}_{\mathrm{LE}}<$ Also hier kommt jetzt das Problem/ ähm erst mal das erste Problem - ich glaub das sagen wir auch noch mal - ähm, ob man ne Übersetzung davon geben soll • - oder nicht ((fragend)). • Ähm ja und dann noch dieses: „Ja auf Folie? Oder Anschreiben? Und ob die das abschreiben sollen.“ Also diese Problematik. [...]> (PG8)

Oftmals wurde vorgegriffen und auf spätere Äußerungen im Handlungsverlauf verwiesen:

$544 \mathrm{~A}_{\mathrm{LE}}<\mathrm{Ja}$. Und mir fällt nichts anderes ein. Und jetzt überleg ich, ob ich s gleich ganz lasse mit diesem Rollenspiel. Also an den Punkt komme ich auch noch mehrfach, aber wir haben dann doch noch ne Möglichkeit gefunden, [...]. Wir kommen ja immer wieder an den gleichen Punkt irgendwie [...].> (PG8)

Aus den vorliegenden Daten lässt sich ableiten, dass die Äußerungen der Teilnehmenden neben erinnerten auch auf postaktionalen Gedanken basieren, die reflektierender, handlungsübergreifender oder kontextualisierender Art sein können. In den LE-Protokollen kommt es durch die Retrospektive vielfach zum Nachdenken über Gesprächsinhalte, Handlungen oder die eigene Person, zum Aktivieren von Gedächtnisinhalten, die über die Handlung hinaus wirksam sind sowie zu Aussagen, die im Prozess der videounterstützten Datenerhebung zur eigenen Orientierung bzw. zu einem besseren Verständnis der aufgezeichneten Handlung durch Dritte beitragen sollen.

\subsection{Zwischenfazit}

Die Datenanalyse zeigt, dass die Äußerungen in den vorliegenden LEProtokollen durchaus in erinnerte und postaktionale Gedanken unterteilt werden können. Eine klare Trennung erwies sich jedoch mitunter als schwierig, da die Grenzen zwischen dem Hier-und-Jetzt und dem zurückliegenden Handlungszusammenhang meist fließend sind und es oftmals innerhalb eines Redebeitrags zu Aussagen kommt, die sich zunächst auf erinnerte Gedanken beziehen und anschließend im weiteren Redeverlauf einen eher postaktionalen Charakter annehmen (z. B. Punkt 5.2.2, Absatz 594). Es scheint, als würden gerade durch den Vorgang des Verbalisierens von Erinnerungen, Gedanken präzisiert bzw. neu konstruiert, d. h. erinnerte Gedanken gehen in postaktionale Gedanken über. Eine eindeutige Zuordnung einzelner Äußerungen zu den zwei Bereichen wird auch dadurch erschwert, dass durch die immanente Adressatenorientierung 
Gedanken nicht nur genannt, sondern beschrieben, erklärt oder begründet werden. Während eine Beschreibung bzw. Erklärung eines Gedanken, die aus Gründen der Verständlichkeit und Kohärenz erfolgt, noch als Erinnerung gelten kann, wird bei Begründungen oftmals auf übergeordnete Strukturen zurückgegriffen, die nicht mehr nur die Handlung selbst betreffen. Henderson/Tallman (2006: 80f.) geben in Bezug auf die Unterscheidung zwischen recall und hindsight thoughts den Hinweis: „The crucial identifier is that the justification of their recalled thoughts had to have occurred during the actual lesson. If they had, then they are correctly designated as recall "there-and-then" thoughts."

Die von den Teilnehmenden bei der Verbalisierung verwendeten Redemittel können, wie oben gezeigt wurde, entsprechende Hinweise geben. Auch das selbstinitiierte Stoppen kann ein Indiz dafür sein, dass Gedanken tatsächlich Handlungsbezug aufweisen. Das Verhältnis der Teilnehmenden untereinander sowie vor allem auch zur datenerhebenden Person ist ebenfalls genauer $\mathrm{zu}$ betrachten. Zum Teil wird es nötig sein, die gesamte Situation, in der ein Gedanke verbalisiert wurde, in den Blick zu nehmen.

Letztlich muss davon ausgegangen werden, dass selten eindeutig festgestellt werden kann, ob die Teilnehmenden tatsächlich Erinnerungen reproduzieren, wie akkurat ihre Erinnerungen sind und ob sie in der Lage waren, ihre Gedanken adäquat in Worte zu fassen. Eine Entscheidung, um welche Art von Äußerung es sich handelt, kann daher immer nur eine Annäherung und der Versuch einer Rekonstruktion sein, die es gut reflektiert zu treffen gilt.

\subsection{Zur Auswertung von Protokollen Lauten Erinnerns}

Es wird immer von der Forschungsfrage, die einer Studie zugrunde liegt, abhängig sein, inwiefern eine strikte Unterscheidung zwischen erinnerten und postaktionalen Kognitionen notwendig ist bzw. welche Art von Gedanken für die Datenauswertung zu verwenden ist.

Ist es das Ziel einer Untersuchung, möglichst handlungsnahe bzw. handlungsleitende Kognitionen zu erheben, um mentale Aktivitäten während einer spezifischen Tätigkeit rekonstruieren zu können, ist es essentiell, vor der eigentlichen Auswertung der Daten jene Aussagen zu identifizieren, die erinnerte Gedanken enthalten und somit in der Datenanalyse zu berücksichtigen sind. Äußerungen, die auf postaktionalen Gedanken basieren wären in diesem Fall aus der Datenanalyse auszuschließen (vgl. Schepens et al. 2007: 463).

Sie können jedoch ebenfalls ausgewertet werden, wenn sie zur Beantwortung der Forschungsfrage beitragen. Postaktionale Gedanken könnten z. B. zusätzlich von Interesse sein, wenn es darum geht, Einstellungen, Überzeugungen, subjektive Sichtweisen oder Wissensbestände einer Person zu erforschen oder Reflexionsprozesse zu untersuchen. Henderson/Tallman (2006) analysierten so- 
wohl die recall als auch die hindsight thoughts, jedoch in voneinander getrennten Analyseschritten. Die postaktionalen Gedanken aus dem Lauten Erinnern wurden wie die reflexiven Daten behandelt, die durch nachträgliche halbstrukturierte Interviews erhoben wurden, um die selbstreflexiven Kompetenzen der Teilnehmenden zu untersuchen (Henderson/Tallmann 2006: 81f.).

Es wäre auch denkbar, erinnerte und postaktionale Gedanken gleichermaßen auszuwerten, wenn es darum geht, generelle Wissensbestände, Einstellungen und Überzeugungen der ForschungspartnerInnen zu erheben, um deren berufliches Selbstverständnis in Bezug auf den jeweiligen Handlungskontext zu erforschen. In diesem Fall wäre eine Unterscheidung in erinnerte und postaktionale Gedanken vermutlich nicht unbedingt nötig.

Ein Teil der retrospektiv erhobenen Daten kann auch dazu beitragen, das Verständnis der Impulsdaten zu vertiefen. In diesem Fall sind neben den erinnerten Gedanken, die Gesagtes noch einmal erläutern, auch jene Äußerungen von Interesse, die Hintergrundinformationen liefern, aktionale oder nonverbale Handlungen erklären oder zur besseren Orientierung im Datenmaterial beitragen.

\section{Zusammenfassung und Ausblick}

Im Fokus dieses Beitrags stand die differenzierte Auseinandersetzung mit retrospektiven Verbalisationsverfahren, die in der forschungsmethodischen Diskussion bisher nur wenig berücksichtigt wurden. Begriffe wie nachträgliches Lautes Denken oder stimulated recall werden vielfach für sehr unterschiedliche Arten retrospektiver Datenerhebungsverfahren verwendet. Es wurde daher neben einer terminologischen Einordnung und Systematisierung verschiedener intround retrospektiver Verfahren detailliert auf das Laute Erinnern eingegangen, das als Erhebungsmethode für die hier verwendeten Daten diente. Die Analyse der Protokolle Lauten Erinnerns konnte verdeutlichen, dass es trotz sorgfältiger Planung und Durchführung der Datenerhebung mit einem expliziten Fokus auf erinnerte Gedanken nicht ausbleibt, dass aus verschiedenen Gründen, wie z. B. Zeitverzögerung, Rezipientendesign und Zusammenhang zwischen Denken und Sprache, auch zahlreiche postaktionale Gedanken geäußert werden. Dennoch ist m. E. an einer Vorgehensweise, wie sie für das Laute Erinnern beschrieben wurde, festzuhalten, um möglichst aussagekräftige Daten zu erheben.

LE-Protokolle sind vielschichtige Datensätze, die je nach Erkenntnisinteresse einer Studie unterschiedliche Forschungsfragen beantworten können. Im Sinne valider Untersuchungsergebnisse bedarf es jedoch zusätzlicher Analyseschritte, die vor der eigentlichen Datenauswertung sicher stellen sollten, dass nur jene Äußerungen analysiert werden, die auch tatsächlich zur Beantwortung der Forschungsfrage beitragen können. Huber/Mandl (1994: 17) betonen, dass es 
notwendig ist, „die eigenen ,forschungsleitenden' Hypothesen zu spezifizieren, d. h. genau anzugeben, wonach man sucht, wenn man sich um Zugang zu handlungsrelevanten Kognitionen bemüht“".

Das Potential retrospektiver Verbalisierungsverfahren liegt darin, interaktive Aushandlungs- und Lernprozesse nicht nur beobachtend oder anhand entstandener Produkte zu untersuchen, sondern indem Untersuchungsteilnehmende ihre Innensicht in Bezug auf ihre mentalen Prozesse während der Ausführung einer Tätigkeit verbalisieren. Außerdem erlauben retrospektive Verbalprotokolle in der Kombination mit anderen Erhebungsverfahren, wie z. B. der Videographie, im Sinne einer Between-Methods-Triangulation tiefere und umfassendere Einblicke in den Untersuchungsgegenstand (Denzin 2000). Die Interaktionsdaten werden so durch erinnerte Gedanken und Gefühle, d. h. durch zusätzliche Informationen aus der Sicht der ForschungspartnerInnen ergänzt und erweitert. Zusätzlich können retrospektive Verbalisationsverfahren zur Validierung der Impulsdaten beitragen.

In einer weiterführenden methodologischen Diskussion werden Aspekte der Analyse retrospektiver verbaler Daten sowie der theoretischen Fundierung introspektiver Verfahren zwischen kognitionspsychologischen und soziokulturellen Paradigmen eine stärkere Rolle spielen müssen. So gilt es beispielsweise weiter auszuloten, welche Auswirkung die aus soziokultureller Perspektive vorherrschende soziale Bedingtheit und Adressiertheit der Äußerungen auf eine angemessene Interpretation der Daten hat.

\section{Literatur}

Aguado, Karin (2004). Introspektive Verfahren in der empirischen Fremdsprachenerwerbsforschung. Methodisch-methodologische Überlegungen. Fremdsprachen und Hochschule 71: 24-38.

Beyer, Sabine (2005). Introspektive Verfahren im fremdsprachlichen Unterricht. Deutsch als Fremdsprache 42/1: 18-22.

Denzin, Norman K. / Lincoln, Yvonna S. (2000). Handbook of qualitative research. Thousand Oaks, California: Sage Publications, 2nd ed.

De Smet, Marijke / Van Keer, Hilde / De Wever, Bram / Valcke, Martin (2012). Studying thought processes of online peer tutors through stimulated-recall interviews. Higher Education 59/5: 645-661.

Dörnyei, Zoltán (2007). Research methods in applied linguistics. Oxford: University Press.

Edwards-Leis, Christine (2006). Variations to stimulated recall protocols to enhance student reflection: I did, I saw, I remembered. Queensland, Australia. Online: http://www.aare.edu.au/06pap/edw06717.pdf (letzter Aufruf [01.10.2011]). 
Ericsson, K. Anders / Simon, Herbert A. (1987). Verbal reports on thinking. In: Færch, Claus / Kasper, Gabriele (Hrsg.): Introspection in second language research. Clevedon: Multilingual Matters. 24-53.

Ericsson, K. Anders / Simon, Herbert A. (1993). Protocol analysis: Verbal reports as data. Cambridge, Mass.: The MIT Press. 2nd, rev. ed.

Færch, Claus / Kasper, Gabriele (Hrsg.) (1987). Introspection in second language research. Clevedon: Multilingual Matters.

Feick, Diana (in Vorbereitung). Autonomes Lernen als Sozialer Prozess. Autonomiepotenziale gruppeninterner Aushandlungsprozesse am Beispiel von Handy-Videoprojekten im DaFUnterricht. Dissertation, Universität Leipzig.

Gass, Susan M. / Mackey, Alison (2000). Stimulated recall methodology in second language research. Mahwah, NJ: Erlbaum.

Heine, Lena (2005). Lautes Denken als Forschungsinstrument in der Fremdsprachenforschung. Zeitschrift für Fremdsprachenforschung 16/2: 163-185.

Heine, Lena / Schramm, Karen (2007). Lautes Denken in der Fremdsprachenforschung: Eine Handreichung für die empirische Praxis. In: Vollmer, Helmut J. (Hrsg.): Synergieeffekte in der Fremdsprachenforschung. Frankfurt am Main: Lang. 167-206.

Henderson, Lyn / Tallman, Julie I. (2006). Stimulated recall and mental models: Tools for teaching and learning computer information literacy. Lanham, Md: Scarecrow Press.

Huber, Günter L. / Mandl, Heinz (1994). Verbalisationsmethoden zur Erfassung von Kognitionen im Handlungszusammenhang. In: Huber, Günter L. / Mandl, Heinz (Hrsg.): Verbale Daten. Weinheim: Beltz. 11-42.

Knorr, Petra (in Vorbereitung). Kooperative Unterrichtsplanungsgespräche angehender Englischlehrender. Dissertation, Universität Leipzig.

Knorr, Petra / Schramm, Karen (2012). Datenerhebung durch Lautes Denken und Lautes Erinnern in der fremdsprachendidaktischen Empirie. In: Doff, Sabine (Hrsg.): Empirisch basierte Methoden in der Fremdsprachenforschung. Tübingen: Narr. 184-201.

Mackey, Alison (2006). Epilogue: From introspections, brain scans, and memory tests to the role of social context: Advancing research on interaction and learning. Studies in Second Language Acquisition 28/2: 369-379.

Mackey, Alison / Gass, Susan M. / McDonough, Kim (2000). How do learners perceive interactional feedback? Studies in Second Language Acquisition 22/4: 471-497.

Pressley, Michael / Afflerbach, Peter (1995). Verbal protocols of reading: The nature of constructively responsive reading. Hillsdale, NJ: Erlbaum.

Raith, Thomas (2011): Kompetenzen für aufgabenorientiertes Fremdsprachenunterrichten. Eine qualitative Untersuchung zur Ausbildung von Fremdsprachenlehrkräften. Tübingen: Narr.

Rehbein, Jochen / Schmidt, Thomas / Meyer, Bernd / Watzke, Franziska / Herkenrath, Annette (2004). Handbuch für das computergestützte Transkribieren nach HIAT. Universität Hamburg: Sonderforschungsbereich Mehrsprachigkeit. (Arbeiten zur Mehrsprachigkeit (Folge B, 56) 
Reitano, Paul / Sim, Cheryl (2010). The value of video in professional development to promote teacher reflective practices. International Journal of Multiple Research Approaches 4: 214-224.

Sasaki, Tomomi (2008). Concurrent think-aloud protocol as a socially situated construct. IRAL - International Review of Applied Linguistics in Language Teaching 46/4: 349-374.

Schepens, Annemie / Aelterman, Antonia / van Keer, Hilde (2007). Studying learning processes of student teachers with stimulated recall interviews through changes in interactive cognitions. Teaching and Teacher Education 23: 457-472.

Schön, Donald A. (1983). The reflective practitioner: How professionals think in action. New York: Basic Books.

Smagorinsky, Peter (1998). Thinking and speech and protocol analysis. Mind, Culture and Activity 5: 157-177.

Smagorinsky, Peter (2001). Rethinking protocol analysis from a cultural perspective. Anпиal Review of Applied Linguistics 21: 233-245.

Swain, Merrill (2006). Verbal protocols: what does it mean for research to use speaking as a data collection tool? In: Chalhoub-Deville, Micheline / Chapelle, Carol A. / Duff, Patricia A. (Hrsg.): Inference and generalizability in applied linguistics: multiple perspectives. Amsterdam: John Benjamins. 97-113.

Vygotsky, Lev S. (1987). Thinking and speech. In: Rieber, R. W. / Carton, A. S. (Hrsg.): The collected works of L. S. Vygotsky: Vol. 1. Problems of general psychology. New York: Plenum Press. 39-285.

Weidle, Renate / Wagner, Angelika C. (1994). Die Methode des Lauten Denkens. In: Huber, Günter L. / Mandl, Heinz (Hrsg.): Verbale Daten. Weinheim: Beltz. 81-103.

Würffel, Nicola (2001). Protokolle Lauten Denkens als Grundlage für die Erforschung von hypertextgeleiteten Lernprozessen im Fremdsprachenunterricht. In: Müller-Hartmann, Andreas / Schocker-von Ditfurth, Marita (Hrsg.): Qualitative Forschung im Bereich Fremdsprachen lehren und lernen. Tübingen: Narr. 163-186.

Wyss, Corinne (2008). Zur Reflexionsfähigkeit und -praxis der Lehrperson. Bildungsforschung $5 / 2$ : 1-5. Online: http://www.bildungsforschung.org/Archiv/2008-02/lehrperson/ (letzter Aufruf [23.08.2011]). 


\title{
„Sehen Sie sich Ines an.“ \\ Zur sozialen Situiertheit des Videobasierten Lauten \\ Erinnerns
}

\section{Diana Feick}

\begin{abstract}
Within the cognitive paradigm of second language research, verbal protocols in the form of think-alouds as well as retrospective reports are regarded as direct representations of cognitive processes. From this perspective, data collection measurements that aim at neutralizing the research context are necessary to obtain valid, non-reactive data. Prior training, absence of the researcher and standardized instruction amongst others are seen as helpful to avoid phenomena like addressivity that might alter cognitive processes while being verbalized. Recent studies in second language research using introspective methods have revealed interactive features in their data which point to the fact that protocols are socially situated constructs. A rationalistic approach towards data collection is therefore to be questioned. Examples from a study on collaborative decision-making processes within a mobile language learning project using videostimulated recall are presented to illustrate nonverbal as well as verbal forms of addressivity in the data. A sociocultural perspective, which is taken in the second part of the article, explains the observed interdependence between data collection, the nature of data and data analysis from its social context. Reactivity as a possible consequence of retrospective verbalization processes can thus be confirmed.
\end{abstract}

\section{Einführung}

Innerhalb der Fremdsprachenforschung erweisen sich retrospektive Datenerhebungsverfahren, insbesondere das (videobasierte) Laute Erinnern (V)LE bzw. (video) stimulated recall, als geeignete Methode, um Kognitionen zu erfassen, auf die mittels simultaner introspektiver Verfahren wie dem Lauten Denken (LD) in bestimmten Untersuchungssettings nicht zugreifbar wäre. $\mathrm{Zu}$ solchen Settings zählen Studien zur Unterrichtskommunikation oder zu fremdsprachenerwerbsspezifischen Interaktionssituationen und -konstellationen, in denen eine parallel zur Handlung stattfindende Verbalisierung von Gedanken kaum oder nicht realisierbar ist und daher nur in Form einer nachträglichen Äußerung erinnerter Gedanken während der Handlung erfolgen kann. In Abgrenzung zum LD stellt (V)LE „die aus dem Langzeitgedächtnis erfolgende nachträgliche, ungefilterte Verbalisierung einer Person von Gedanken während einer (mentalen, interaktionalen oder aktionalen) Handlung" dar (Knorr/Schramm 2012), wobei diese Erinnerungen aus Kognitionen, Emotionen und Motiven gespeist sind (Dörnyei 2007).

Je nach zugrundeliegender Kognitions- und Wissenschaftstheorie bestehen differierende Überzeugungen zu Generierungsbedingungen, Beschaffenheit und Funktion von LD- bzw. VLE-Daten, wobei meist nicht ausdifferenziert wird, 
inwiefern diese Annahmen jeweils einschränkungslos von einer auf die andere Datenerhebungsmethode und die daraus resultierenden Datentypen übertragbar sind.

Hinsichtlich der Generierungsbedingungen bilden den Auslöser für die folgenden Ausführungen Überlegungen bezüglich des Mehrwerts der Datenerhebungsvorbereitung und des Trainings des VLE. Beide erhebungsvorgelagerten Maßnahmen gelten neben standardisierter Instruktion, Zurückhaltung bzw. Abwesenheit der Forschungsperson während des Verbalisierens, der freien Wahl der Verbalisierungssprache sowie einer kürzestmöglichen Zeitspanne zwischen Impulshandlung und Impulsdarbietung als förderlich zur Sicherstellung einer höchstmöglichen Datenqualität (u. a. Gass/Mackey 2000 für das (V)LE, Knorr/ Schramm 2012). Besonders ein vorgelagertes Training soll dazu beitragen, die mögliche Fremdadressiertheit der Äußerungen, also eine reflexive, deskriptive oder explizierende Ausrichtung, so gering wie möglich zu halten, bestenfalls zu vermeiden (Ericsson/Simon 1998, Heine/Schramm 2007). Diese Empfehlungen treffen jedoch vornehmlich auf das LD zu, sodass zum Mehrwert des Trainings für das (V)LE im Allgemeinen und bezüglich der Adressiertheitsreduktion im Speziellen nur wenige Aussagen zu finden sind. Meist wird verallgemeinernd das vorherige Üben als nicht zwingend nötig erachtet (Gass/Mackey 2000, Henderson/Tallmann 2006).

Im Rahmen eines laufenden Dissertationsprojektes, welches zur Vorbereitung der Datenerhebung mittels VLE alle oben genannten Maßnahmen umsetzte, zeigten sich in den Daten Phänomene, die vermuten lassen, dass beim VLE Merkmale der Adressiertheit wiederzufinden sind. Daraus ergibt sich die Frage, ob die eingesetzten Datenqualitätsmaßnahmen nicht zielführend waren oder die beobachtete Adressiertheit möglicherweise ein den Introspektions- und Retrospektionsdaten inhärentes Charakteristikum darstellt.

Im Folgenden soll nach einem kurzen Überblick über kognitionstheoretische Vorannahmen zu Verbalprotokollen (Abschnitt 1) an Datenbeispielen die soziale Situiertheit von VLE-Daten veranschaulicht (Abschnitt 2) und unter Rückgriff auf die soziokulturelle Theorie des Lernens begründet werden (Abschnitt 3). Dies ermöglicht es, Rückschlüsse für Potenziale und Grenzen der Datenerhebungsvorbereitung zu ziehen und die Berücksichtigung dieser Faktoren bei der Datenanalyse aufzuzeigen (Abschnitt 4). Der Beitrag schließt mit einem Ausblick auf die sich aus den Überlegungen ableitenden forschungsmethodologischen Konsequenzen.

\section{Kognitionstheoretische Grundlagen}

Die klassische, kognitivistische Sicht auf Verbalprotokolle geht auf die Psychologen Ericsson/Simon (1993) zurück, nach deren Auffassung Kognitionen im 
Gehirn als Informationsverarbeitungsprozesse ablaufen, wobei sie aus dem Arbeitsspeicher abrufbar sind und durch ihre Versprachlichung erkennbar werden können. Innerhalb des Kognitivismus gilt Sprache als bloßes Kommunikationswerkzeug und stellt somit die direkte Wiedergabeform von Gedanken dar. Versprachlichte Repräsentationen von Kognitionen können nach diesem Verständnis gleichzeitig oder nachtäglich zu einer Handlung abgerufen werden und dabei nach Ericsson/Simon (1993: 16ff.) drei Arten von Daten erzeugen. Unter level1-Daten verstehen sie Kognitionen, die im Sinne des inneren Sprechens bereits verbal kodiert im Kurzzeitgedächtnis vorliegen (talk alouds) und nicht mehr in Sprache überführt werden müssen. Daten auf level 2 (think alouds) hingegen stellen Kognitionen dar, die nonverbal vorliegen und zur Äußerung erst in einen verbalen Code überführt werden müssen, beispielsweise Gerüche oder Emotionen. level-3-Daten (ebenfalls think alouds) beinhalten schließlich in Abgrenzung zu Daten des zweiten Levels Beschreibungen, Erklärungen, Begründungen, Theoretisierungen oder Interpretationen zu den abgerufenen Kognitionen. Der letzte Datentyp stellt nach Ericsson/Simon (1998) somit keine zuverlässige Datenart zum Erfassen von Kognitionen dar, weil die zusätzlichen Verarbeitungsprozesse, die mit dem an Dritte adressierten Erklären von Gedanken einhergehen, die abgerufen Informationen in ihrer Beschaffenheit beeinflussen und sogar verändern können.

Die Grundannahme für den Erhalt valider, nichtreaktiver, also level-1- und -2Daten, besteht darin, Untersuchungsteilnehmende derart zu unterweisen, dass die Beeinflussung der kognitiven Prozesse durch das gleichzeitige laute Äußern der Gedanken verhindert wird. Für an Ericsson/Simon (1993) angelehnte Forschungsdesigns stellt die Datenerhebungsvorbereitung somit ein kleinschrittig festgelegtes, standardisiertes, idealerweise unter Laborbedingungen durchgeführtes Prozedere dar, mit dem Ziel, die Kontextfaktoren weitgehend auszuschalten oder zumindest zu kontrollieren. So werden Empfehlungen (u. a. Heine 2005, Heine/Schramm 2007, Knorr/Schramm 2012) bezüglich der Instruktion, der Demonstration, des Trainings und der Durchführung gegeben, die das Erheben von Daten, welche die Verbalisierung von (erinnerten) Kognitionen im Sinne von Level 2 darstellen, fördern sollen. Der diesbezügliche Nutzen eines vorgelagerten Trainings zur Optimierung der Datenerhebung beim VLE konnte hierfür bisher nicht empirisch belegt werden.

Diesen Vorannahmen zufolge sind (V)LE-Daten in ihrer Validität LD-Daten immer nachgeordnet, weil die Akkuratheit der abgerufenen Gedanken bzw. Erinnerungen mit zunehmender Zeitspanne zwischen Auftreten des Gedankens und seiner Verbalisierung nachlässt, da Erinnerungen oft mit Hilfe eines erinnerungsfördernden Videoimpulses erst aus dem Langzeitgedächtnis (LZG) abgerufen und $\mathrm{zu}$ ihrer Verbalisierungen ins Kurzzeitgedächtnis transferiert werden müssen. Neu aufgenommene Informationen liegen im LZG jedoch mit bereits existierenden mentalen Modellen, Schemata oder anderen Formen der 
Wissensrepräsentation verknüpft vor (vgl. Henderson/Tallmann 2006: 59), sind also u. U. nicht getrennt voneinander verbalisierbar. Gass/Mackey (2000) gehen dabei mit Bezug auf Bloom (1954) von einer Spanne von 48 Stunden aus, in der Erinnerungen noch mit einer Genauigkeit von $95 \%$ wiedergegeben werden können, wobei dies nur Erinnerungen an die Kognitionen betrifft, die ins LZG aufgenommen wurden, die also nicht (sofort) vergessen wurden.

Der Einbezug des LZG in den Verbalisierungsprozess führt nichtsdestotrotz zu einer Beschaffenheit der Daten, bei der die Unterscheidung in Typ 2 und 3 aus kognitionspsychologischer Perspektive zu kurz greift. Besonders das Phänomen der Adressiertheit, dem sich der folgende Abschnitt widmet, ist für Gedankeninhalte, die durch VLE erhoben wurden, neu zu betrachten.

\section{Elemente der Adressierung in VLE-Daten}

\section{$2.1 \quad$ Forschungsprojekt}

Bevor unter 2.2 und 2.3 detailliert die Adressiertheit als Merkmal von sozialer Situiertheit anhand ausgewählter VLE-Daten dargestellt wird, soll im Folgenden ein kurzer Überblick über das Dissertationsprojekt gegeben werden, in dessen Rahmen die Datenerhebung mittels VLE stattfand.

Den Widerspruch zwischen autonomieförderlichen Lernumgebungen und den realen, oft autonomiehemmenden institutionellen Lernkontexten als Ausgangspunkt nehmend, besteht das Erkenntnisinteresse der Studie darin herauszufinden, wie das projektorientierte Deutschlernen mit mobilen Endgeräten dazu beitragen kann, dass Autonomiepotenziale von Gruppen durch und in Sprachlernprozessen freigesetzt und reflektierbar werden. Innerhalb des Lernkontextes „Unterrichtsprojekt“, das durch seine Offenheit vielfältige Formen der Mitbestimmung zulässt, wird daher anhand von videographisch erhobenen Daten interaktionsanalytisch untersucht, wie sich während eines Handyvideoprojekts im mexikanischen Deutsch-als-Fremdsprache-Unterricht gruppeninterne Aushandlungen zur Entscheidungsfindung gestalten. Zusätzlich soll durch videobasiertes Lautes Erinnern beim Sichten der Videomitschnitte erkundet werden, wie die einzelnen Gruppenmitglieder diese Prozesse hinsichtlich ihrer Mitbestimmung wahrnehmen. Dadurch werden die LE-Daten den Interaktionsdaten im Sinne einer methodenübergreifenden Triangulation validierend gegenübergestellt. Das vorliegende Dissertationsprojekt möchte somit zur empirischen Erfassung des Konstrukts ,soziale Autonomie' beitragen, indem es Autonomiepotenziale in Gruppeninteraktionsprozessen ergründet, diese mittels spezifischer Interaktionsmuster in gruppenbasierten Lernarrangements identifiziert und beschreibt, um daraus Hypothesen für die Anbahnung und den Einsatz autonomie- und damit sprachlernförderlicher Gruppeninteraktionen im Fremdsprachenunterricht abzuleiten. 
Aus diesem Erkenntnisinteresse leitet sich die zentrale Forschungsfrage mit zwei Teilfragen ab:

Welche Potenziale von sozialer Autonomie bietet der Projektunterricht mit mobilen Endgeräten in Deutsch-als-Fremdsprache-Kursen?

- Wie gestalten sich Entscheidungsfindungsprozesse in einem Handyvideoprojekt während der polyadischen Interaktion?

- Wie werden diese von den einzelnen Gruppenmitgliedern (als Zeichen ihrer Mitbestimmung) wahrgenommen?

Die für die Studie zugrundeliegende Datenerhebung (vgl. Feick 2012: 202-217) erfolgte in einem studienbegleitenden Deutsch-als-Fremdsprache-Kurs an der Universität von Guadalajara/Mexiko und in einem Spracheninstitut der gleichen Stadt während eines fünfwöchigen Handyvideoprojekts, im Rahmen dessen Lernende eigene, maximal fünfminütige Videoclips zu einem frei gewählten Thema produzierten.

Die während des Projektes videographisch erhobenen Daten dienten als Videoimpulse für das individuelle Laute Erinnern aller Gruppenmitglieder im Anschluss an Sitzungen mit hoher Entscheidungsdichte, d. h. in der Planungs-, Postproduktions- und Auswertungsphase des Projekts. Die mittels einer ersten Samplingphase gewonnenen Entscheidungssequenzen der Interaktionsdaten wurden nach dem Verfahren der Halbinterpretativen Arbeitstranskription (HIAT) im EXMARaLDA Partitur Editor im Verhältnis 1:25 grob- und nach einem weiteren ereignisgeleiteten Sampling im Verhältnis 1:80 feintranskribiert sowie anschließend im Hinblick auf Muster der Entscheidungsfindung sowie unter Rückgriff auf die Individualdaten des Lauten Erinnerns analysiert und interpretiert.

Um im Sinne der Empfehlungen zum Erhalt möglichst valider, nichtreaktiver Daten des Typs 2 nach Ericsson/Simon (1984, 1993) (siehe Abschnitt 1) beim Einsatz des VLE vorzugehen, wurden diese für die Datenerhebungsvorbereitung und die Durchführung der Erhebung umfassend berücksichtigt (vgl. Feick 2012: 206 ff.). Hierfür fand ein Training der Untersuchungsteilnehmenden während der Erhebungsvorbereitungsphase mittels einer Bild-Text-Zuordnungsaufgabe statt. Sie war jeweils paarweise zu bearbeiten, wobei der Lösungsprozess videographisch festgehalten wurde. Der Mitschnitt diente anschließend als Vorlage für die Erprobung des individuellen LE. Während des auf das VLE folgenden Feedbacks der übrigen Gruppenteilnehmenden und der Forscherin konnte gezielt auf von einigen Lernenden geäußerte unerwünschte Abweichungen in der Verbalisierungsweise wie allgemeine Beschreibungen des gerade Gesehenen oder der Äußerung von ad hoc-Gedanken, die erst das Betrachten des Mitschnittes auslösten, aufmerksam gemacht werden.

Die Instruktion für das VLE erfolgte nach einem standardisierten Verfahren mit dem ausdrücklichen Hinweis, dass nur Gedanken während der im Video 
sichtbaren Handlungen von Interesse seien. Die Durchführung des Lauten Erinnerns war dementsprechend von der freien Wahl der Verbalisierungssprache, der räumlich abgetrennten Positionierung der Untersuchungsleitenden und dem daraus resultierenden selbstinitiierten Stoppen des Mitschnitts geprägt, wobei die Durchführung des VLE binnen 48 Stunden nach Aufzeichnung des Impulsmaterials, meist jedoch direkt im Anschluss an die entsprechende Projektgruppensitzung bzw. an die Arbeitsphase, die als Impulssequenz diente, stattfand.

Nach dieser Beschreibung der Entstehungsbedingungen der VLE-Daten folgt in den kommenden zwei Abschnitten eine eingehende Analyse der Beschaffenheit des Datenmaterials hinsichtlich der Elemente sozialer Situiertheit.

\subsection{Adressierung auf nonverbaler Ebene}

Merkmale der Adressierung sind in den als Beispiel herangezogenen Datensätzen aus dem unter 3.1 beschriebenen Forschungsprojekt sowohl auf verbaler (2.3) als auch auf nonverbaler Ebene festzustellen. Nonverbal äußert sich die Adressierung vor allem im Blickverhalten der Untersuchungsteilnehmenden. Im Rahmen der Datenerhebung waren die Deutsch-als-Fremdsprache-Lernenden dazu aufgefordert, die Impulssequenzen an einem Notebook mit integrierter Webcam anzusehen, welche alle Äußerungen und Handlungen im Aufnahmeradius der Kamera, d. h. die vor dem Gerät sitzende Person im Bildausschnitt Kopf und Brust, aufzeichnete (vgl. Abb. 1-4 ${ }^{1}$ ). Bei der Unterbrechung der Aufnahme wandten sich etwa die Hälfte der Teilnehmenden permanent oder in regelmäßigen Abständen in ihrer Sprechhaltung an die Kamera, so als würden sie sich an ein potenzielles Gegenüber richten oder auch einen (zukünftigen) Empfänger ihrer Äußerungen antizipieren. Dies verdeutlichen der direkte Blickkontakt (Abb. 1-4) sowie die mit den Verbalisierungen einhergehende Gestik (Abb. 2 und 4). Die beschriebenen nonverbalen Phänomene wiederholten sich bei den Teilnehmenden im Laufe beider zur Datenerhebung vorgenommenen VLE-Sitzungen.

Dass ein solcher Augenkontakt vermutlich nicht nur ein unbewusstes, ungesteuertes Umherschweifen des Blickes darstellt, welcher möglicherweise der frontalen Aufnahmekonstellation geschuldet sein könnte, sondern eine bewusste Adressierung eines (abwesenden) Gegenübers, verdeutlichen die Videostandbilder in Abb. 3 und 4. In diesen Fällen kam anstelle der Webcam eine externe Kamera zum Einsatz, die im 45-Grad-Winkel neben dem Computer positioniert wurde und die VLE-Daten aufzeichnete. Während dieser Mitschnitte wandten sich die Untersuchungsteilnehmer in ihrer Sprechhaltung direkt, also bewusst und wiederholt an die Kamera und nutzten somit intentional den Aufnahmemodus des Mediums zur Adressatenansprache bei der Verbalisierung ihrer

1 Der Abdruck erfolgt mit freundlicher Genehmigung der abgebildeten Untersuchungsteilnehmenden. 
erinnerten Gedanken. In Abbildung 4 wird zusätzlich deutlich, dass der Forschungspartner mit einer Handgeste auf eine explizite Stelle des Videos verweist, auf die er sich in seiner an die Kamera gewandten Verbalisierung bezieht.

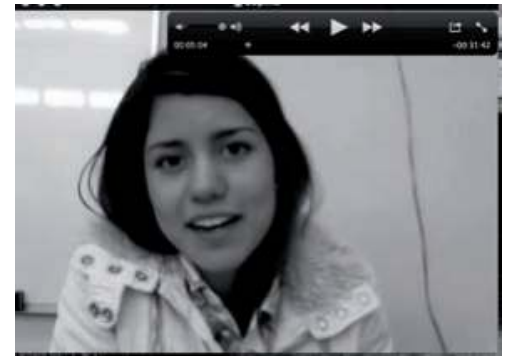

Abbildung 1: Standbild mit direktem Kamerablickkontakt

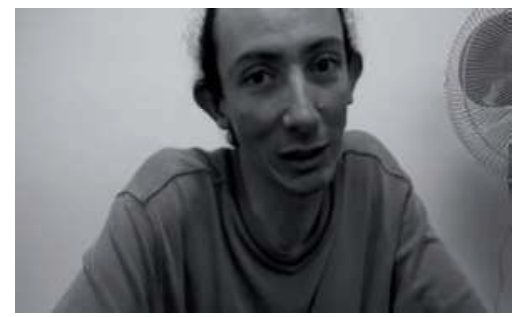

Abbildung 3: Standbild mit direktem Blickkontakt zu externer Kamera

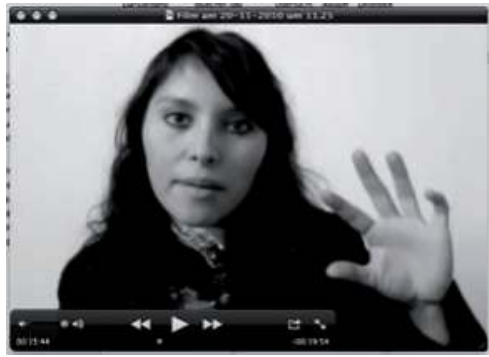

Abbildung 2: Standbild mit direktem Kamerablickkontakt und Handgeste

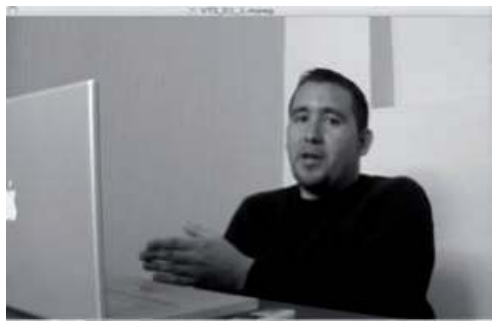

Abbildung 4: Standbild mit direktem Blickkontakt zu externer Kamera und Handgeste

An den aufgeführten Beispielen wird somit ersichtlich, dass auch trotz Abwesenheit der Forschenden, welche den Grad der Fremdsteuerung und Fremdadressierung potenziell verringern sollte, durch wiederholte mimische und gestische Signale wie Blickkontakt, Handzeichen, u. ä. von den Teilnehmenden bewusst (oder unbewusst) imaginäre Empfänger ihrer Botschaften angesprochen bzw. zukünftige Rezipienten ihrer Äußerungen antizipiert werden und somit von ihnen eine Situation mit Gesprächscharakter konstruiert wird.

\subsection{Adressierung auf verbaler Ebene}

Neben den im vergangenen Abschnitt beschriebenen nonverbalen Merkmalen der Adressierung findet sich dieses Phänomen auch auf verbaler Ebene wieder. Idealerweise, also im Sinne des Datentypens 2 nach Ericsson/Simon (1993), gibt die Verbalisierung die bloße Kognition ohne zusätzliche kontextualisierende Redeanteile wieder. Für das VLE hieße dies, dass dabei die Kognitionen in 
Form von Erinnerungen an Gedachtes aus dem LZG geäußert werden, wie das folgende Datenbeispiel verdeutlicht:

Datensatz LARA_G3_0611:

18:30: Pensé: qué bueno, JUAN está de acuerdo conmigo.

Ich dachte: wie gut, JUAN ist mit mir einverstanden ${ }^{2}$

In der Regel bilden ausschließlich auf das Erinnerte beschränkte Äußerungen wie diese jedoch die Ausnahme im vorliegenden Datenmaterial; vielmehr sind die verbalisierten Erinnerungen in kontextualisierende Redeanteile eingebettet.

Das Phänomen der Adressierung ist dabei als soziale Situierung in Form von Kontextualisierungen oder der bereits erwähnten Konstruktion einer Gesprächssituation zu verstehen. Es tritt beispielsweise als direkte Adressatenansprache mittels Imperativsätzen oder Rückversicherungspartikeln sowie als Kommentar auf Text- oder Metaebene auf. Diese verschiedenen Kategorien ${ }^{3}$ werden im Folgenden anhand ausgewählter Beispiele, welche durch Unterstreichungen vorgehoben sind, innerhalb ihres Äußerungskontextes vorgestellt.

\subsubsection{Adressatenansprache}

a) durch Imperativsatz

Datensatz NERO_G1_1911:

16:26: De hecho, nuestro lenguaje corporal es bastante de frustración. Vean a $L U L O$ tocándose el cuello y yo con los brazos cruzados.

Auch zeigt unsere Körpersprache eine ziemliche Frustration. Sehen Sie sich (bzw. seht euch, Anm. D.F.) LULO an, wie er sich an den Hals greift und ich mit verschränkten Armen.

Der Untersuchungsteilnehmer wendet sich bezüglich seiner Beobachtungen zur Körpersprache der Gruppenmitglieder mittels eines Imperativsatzes an den oder die imaginierten Adressaten und fordert diese(n) auf, seine Beobachterposition einzunehmen, indem er auf die Videoaufnahme verweist.

b) durch Rückversicherungspartikel

Datensatz JUAN_G3_0611:

01:20: $\quad$ Y pues bueno siempre hay dificultad ponerse de, de acuerdo en equipo, no?

Und also gut es ist immer schwer, sich in einer Gruppe zu, zu einigen, nicht?

\footnotetext{
${ }^{2}$ Diese und alle folgenden Übersetzungen aus dem Spanischen wurden von der Autorin vorgenommen.

${ }^{3}$ Für den Austausch zur Entwicklung terminologisch präziser Kategorienbezeichnungen gilt mein Dank Dr. Simone Amorocho.
} 
Diese Form des Rückversicherungssignals dient aus gesprächsanalytischer Sicht der Aufrechterhaltung des Dialogs, der Verständnissicherung oder der Versicherung der Zustimmung des Adressaten. Hier wird es eingesetzt, obwohl dem Sprecher bewusst ist, dass er mit keiner verbalen oder nonverbalen Bestätigung eines Gegenübers zu rechnen hat.

\subsubsection{Kommentare}

a) Textkommentar

Datensatz NERO_G1_1911:

16:26: Ahí seguíamos con lo del título, que como vuelvo a mencionar, fue de los procesos más complicados.

Hier machten wir mit dem Titel weiter, was, wie ich bereits erwähnt habe, einer der kompliziertesten Prozesse war.

19:42: $\quad$ Y bueno, yo había propuesto un título, pero como lo digo, LULO no estaba contento con ningún título, es la verdad.

Gut, ich hatte einen Titel vorgeschlagen aber wie ich schon sagte, LULO gefiel keiner der Titel, das ist die Wahrheit.

Der Begriff des Textkommentars geht auf Fandrych/Gräfen (2001) zurück. Diese Art der Kommentare stellen Kohärenz- und Kohäsionsmittel zwischen verschiedenen Redeteilen dar. Sie dienen der Bezugnahme und der Diskursstrukturierung für den Produzenten wie auch für den (intendierten) Rezipienten des mündlichen Textes.

b) Metakommentar

Datensatz LULO_G1_1911:

13:57: $\quad$ Me da vergüenza mi actuación. No hay más que decir.

Mein Schauspiel ist mir peinlich. Mehr gibt es nicht zu sagen.

Datensatz INES_G1_2611:

12:10: Ah, pensé que / es que estábamos hablando / bueno, como se puede ver, estábamos hablando de como quién tenía que evaluarnos, entonces, pues pensé que no era posible que alguien de / que era necesario que cada uno se evaluara y que, que no necesariamente con un número. Ahi creo que estoy describiendo lo que estoy viendo, bueno.

Ah, ich dachte, dass / wir sprachen/gut / wie man sehen kann, sprachen wir darüber wer uns zu evaluieren hatte, so, also dachte ich, dass es nicht ging, dass jemand von / dass es nötig war, dass jeder sich selbst evaluiert und dass, dass nicht notwendigerweise mit einer Zahl. Nun glaube ich, dass ich beschreibe, was ich sehe, gut. 
Datensatz SONJA_G3_0611:

02:56: $\quad$ Estaba pensando hacer algo con Frida Kahlo, pero solo lo pensé rápido.

Ich dachte daran, etwas mit Frida Kahlo zu machen, aber das dachte ich nur schnell.

Die hier aufgeführten Kommentare beziehen sich auf einer Metaebene auf den Vorgang des Lauten Erinnerns, indem die Untersuchungsteilnehmenden ihren Verbalisierungsprozess oder im Beispiel Sonjas ihren Denkprozess reflektieren. Im Beispiel Lulo signalisiert er einem abwesenden Adressaten den aus seiner Sicht ausreichenden Umfang seiner Äußerung. Ines nimmt im ersten Segment direkten Bezug auf die Videoaufnahme, womit sie eine Kenntnis selbiger durch den Adressaten ihrer Verbalisierungen annimmt. Zusätzlich kommentiert sie im zweiten Segment ihre Erkenntnis über eine laut der Instruktion zum VLE unerwünschte Form der nachträglichen Äußerung.

Datensatz JUAN_G3_0611:

01:20: $\quad$ En este momento lo que esta pasando es un poco de äh por qué no podemos movernos un poquito mas rapido. A qué me refiero con que mas rápido? En el sentido de sabemos que / tenemos que hacer un video.

Was in diesem Moment passiert ist äh warum können wir nicht ein bisschen schneller vorankommen. Was meine ich mit schneller? In dem Sinne, dass wir wissen, dass / wir müssen ein Video machen.

In diesem Datenauszug richtet sich der Untersuchungsteilnehmer mit einer direkten Frage an den antizipierten Adressaten und nutzt die Frage dabei als Gliederungsmittel. Die damit gewonnene Strukturierung seiner Äußerungen dient damit zum Einem seinem eigenen Verbalisierungsprozess und zum Anderen der Rezipientenführung.

Die hier dargestellten Metakommentare, Gliederungs- und Hörersignale sowie Gesprächspartikeln belegen beispielhaft linguistisch-diskursiv verankerte Merkmale des Dialogischen (Schwitalla 2001) in ursprünglich eher monologisch geltenden VLE-Daten. Diese unter Gesprächsforschern auch als Kontaktparenthesen zusammengefassten Elemente dienen der „Aufrechterhaltung, Prüfung und Beendigung des physischen und psychischen Kontaktes zwischen den Kommunikationspartnern“ (Bayer 1973: 105, zitiert nach Schwitalla 2001: 899).

Im Falle des VLEs richten sich die Untersuchungsteilnehmenden jedoch an ein (meist abwesendes) Gegenüber. Sie beziehen dieses mit den ihnen zur Verfügung stehenden diskursiven Mitteln in ihren Monolog ein, sodass dieser von interaktiven Elementen durchzogen und von Kontextualisierungen geprägt ist. Dadurch wird deutlich, dass sich die Untersuchungsteilnehmenden der Situation „Datenerhebung“ durchaus bewusst sind und diese in ihrem verbalen und nonverbalen Verhalten widerspiegeln. 
In den Daten lässt sich somit eine Interaktivität beobachten, der in ihrer Anlage mittels des Erhebungsdesigns und eines vorgelagerten Trainings ursprünglich entgegengewirkt werden sollte. Es muss sich also bei VLE-Daten um eine Datenart handeln, der das Phänomen der Adressierung inhärent ist. In diesem Zusammenhang ist zu bedenken, dass beim VLE Kognitionen in Form von Erinnerungen im Gegensatz zum LD immer aus dem LZG stammen, eine Anbindung von neuen mit bereits existierenden Inhalten des LZG, also die Voraussetzung für die Erinnerbarkeit von Kognitionen, darstellt. An vergangene Ereignisse gekoppelte Gedanken werden daher aufgrund dieser Verknüpfung mit älteren Wissensbeständen vermischt wiedergegeben, sodass die mit der Fremdadressierung z. T. einhergehende deskriptive und reflexive Art der Äußerungen nicht vermeidbar ist. In den meisten Fällen ist die Verbalisierung der Erinnerungen begleitet von einer Beschreibung oder Erklärung der Situation (s. Knorr in diesem Band), welche diese nicht zwangsläufig zum Datentyp 3 nach Ericsson und Simon (1993) macht, da die Erinnerung einer natürlichen Gesprächssituation entsprechend zwar für den antizipierten Adressaten kontextualisiert, jedoch nicht immer auch automatisch reflektiert oder interpretiert wird.

Nach einer Kurzvorstellung von zwei Studien, in denen weitere Formen der Rezipientenorientierung aufgezeigt wurden, widmet sich der folgende Abschnitt einer genaueren Auseinandersetzung mit den aufgezeigten Phänomenen aus soziokultureller Perspektive.

\section{Die soziokulturelle Sicht auf Verbalprotokolle}

Hinweise zur Erklärung der in den Daten vorgefundenen Auffälligkeiten liefert die Zuhilfenahme der soziokulturellen Perspektive auf LD- und (V)LE-Daten. Vertreter dieses lerntheoretischen Ansatzes gehen davon aus, dass Verbalprotokolle sozial situierte Konstrukte, also interaktiv konstituiert sind und damit auch immer eine Rezipientenorientierung aufweisen (Deschambault 2011, Sasaki 2008, Smagorinsky 1998, 2001, Swain 2006, Witte/Cherry 1994). Der Problematisierung der sozialen Situiertheitsphänomene widmen sich die im Folgenden beschriebenen Studien, welche sich beide des LD als Erhebungsinstrument bedienen.

Smagorinsky berichtet 1997 erstmalig im Rahmen einer Fallstudie zum Schreiben von Rezipientenorientierung seitens des Untersuchungsteilnehmenden, der neben der persönlichen Ansprache des Forschenden (oder seiner Vorstellung dessen) auch sein Sprachregister entsprechend dem sozialen Hierarchieunterschied zum imaginierten Empfänger der Daten anpasste. Sasaki (2008) beschreibt anhand der Kategorien Adressiertheit, Verhaltenserklärungen, Anredeformen und Sprachwahl Ausprägungen der sozialen Situiertheit innerhalb einer 
Metastudie mittels zum Teil ähnlicher Parameter wie den in Abschnitt 2.3 gewählten. Die Adressiertheit trotz Abwesenheit der Forschenden belegt Sasaki anhand der Namensnennung der Forscherin seitens der Untersuchungsteilnehmenden sowie anhand von Fragen und Entschuldigungen, die an das vorgestellte Gegenüber gerichtet sind. Des Weiteren beobachtete die Forscherin bei ihren ProbandInnen Erklärungen des eigenen Verhaltens während des LD v. a. mittels Metakommentaren oder Interaktionspartikeln, wie sie auch in den Daten des vorliegenden Forschungsprojektes (Abschnitt 2.3) vorzufinden waren. $\mathrm{Zu}$ sätzlich verweist sie auf ein sprachenspezifisches Phänomen der Adressierung anhand der häufigen Wahl einer im Japanischen die Formalität einer Situation kennzeichnenden Höflichkeitsform. Abschließend verdeutlicht Sasaki hinter der Sprachwahl zur Ausführung der Verbalprotokolle eine Entscheidung der Forschungsteilnehmenden in Rücksicht auf die Sprachkompetenzen der Untersuchungsleiterin. Am Beispiel einer Studie von Robinson (1992) zeigt Sasaki die sprachspezifische Rezipientenorientierung auf, indem sich Lernende bei freier Sprachwahl mit dem Wissen um die fehlende L1-Sprachkompetenz seitens der/des Forschenden für ihre L2 zum Verbalisieren entschieden.

Mit Blick auf diese in den LD-Daten entdeckten Phänomene wird Sprechen als soziale Handlung verstanden, für dessen Charakterisierung Smagorinsky (2001: 237ff.) zwei Konzepte Bakhtins heranzieht - die Dialogizität (dialogism) und die Adressiertheit (addressivity). Jede Form des Sprechens ist demnach dialogisch, weil dabei auf vorhergehende (auch eigene) Äußerungen aufgebaut wird und sich diese gleichzeitig auf darauffolgende Äußerungen auswirken. Sprechen ist somit immer auch an einen an- oder abwesenden Adressaten gerichtet, unabhängig davon, ob dieses auf eine spezifische Person abzielt oder unspezifisch bleibt. Die dem Sprechen inhärente soziale Komponente kann demnach zu einer, versteckten Dialogizität' (hidden dialogicalitiy) (Bakhtin 1984, zitiert nach Smagorinsky 2001: 238) führen, indem der Sprecher einen Hörer seiner Äußerungen antizipiert, auch wenn dieser, wie im unter Abschnitt 2 vorgeführten Erhebungssetting, nicht physisch präsent ist.

Zur theoretischen Begründung dieser Phänomene berufen sich Vertreter der soziokulturellen Perspektive auf die Annahme, dass Kognition sozial vermittelt ist und daher nicht von deren sozialen, kulturellen und interpersonalen Einflussfaktoren getrennt untersucht werden kann (Deschambault 2011, Sasaki 2008, Smagorinsky 1998, 2001, Swain 2006, Witte/Cherry 1994). Das soziokulturelle Kognitionsverständnis orientiert sich an den Arbeiten von u. a. Wygotski (1934, 2002), Leont'ev (1981) und Wertsch (1981) und grenzt sich somit von der Annahme der Informationsverarbeitungstheoretiker Ericsson und Simon (1993, 1998), dass Sprache die direkte Repräsentation von Kognitionen darstelle (s. Abschnitt 1), ab. Sprechen ist aus soziokultureller Sicht kein bloßes Abrufen von Gedächtnisinhalten, also das Hörbarmachen von vorliegenden Gedanken, sondern verhilft den Gedanken zu ihrer Existenz und ihrer Form: „Das Sprechen 
dient nicht als Ausdruck des fertigen Gedanken. Indem sich der Gedanke in Sprechen verwandelt, gestaltet er sich um, verändert er sich. Der Gedanke drückt sich im Wort nicht aus, sondern vollzieht sich im Wort.“ (Wygotski 2002: 401). Dabei ist der Weg vom verbalen Denken zum Sprechen u. U. ein dynamischer Prozess, der mehrere Ebenen durchläuft: Ein Motiv bringt einen Gedanken hervor und formt ihn aus, er verwandelt sich in inneres Sprechen, dieses wird mit Wortbedeutungen des äußeren Sprechens (= Sinn eines Wortes in einem spezifischen Kontext) versehen und führt schließlich zum äußeren Sprechen, also der lautlichen Artikulation. Ein Gedanke ist also nie mit der ausgesprochenen Wortbedeutung identisch, sondern stellt eher ein übergeordnetes Ganzes dar, das durch seine Umwandlung in Worte (Artefakte) nur eine Verallgemeinerung erfahren kann. Wygotski (ebd.) benutzt hier die Metapher einer Wolke (Gedanke), aus der es einzelne Tropfen (Wörter) regnet und die durch Wind (Motiv) angetrieben wird. Da Denken und Sprechen in wechselseitiger Beziehung stehen, kann das Sprechen gleichzeitig zur Ausformung bzw. Veränderung von Gedanken führen: „Language is data, and with language we are able to manipulate ideas, re-organize them, reshape them, transform them, and construct new ones" (Swain 2006: 101). Im Sinne von Wygotskis Auffassung der kindlichen Entwicklung von Denken und der dabei ablaufenden Umwandlung des äußeren - sozialen - Sprechens in egozentrisches und ab dem Schulalter in inneres Sprechen wirkt es also auf das Denken zurück, indem sich beim Sprechen Gedanken herauskristallisieren, schärfen und dadurch ggf. Inkonsistenzen offengelegt werden. Verbalisierungen regulieren, organisieren und fokussieren die eigenen mentalen Aktivitäten und können somit Kognitionen verändern und dadurch Lernprozesse (Internalisierungen) auslösen (ebd.). Die beim Sprechen stattfindende laute Verbalisierung von Gedanken oder von Erinnerungen an diese kann daher nicht mit LD bzw. LE gleichgesetzt werden.

Diese Überlegungen führen Vertreter der SKP auf Verbalprotokolle zu der Auffassung, dass diese Form der Datenerhebung nicht wie im Sinne der Informationsverarbeitungsansätze Daten hervorbringt, welche deckungsgleich die Kognitionen repräsentieren, die die Untersuchungsteilnehmenden während ihrer Aufgabenbearbeitung oder Interaktion abrufen. Vielmehr wird von einer Beeinflussung der Kognitionen durch das Verbalisieren ausgegangen:

They [Verbalprotokolle, Anm. D.F.] exert this influence in three ways. First, the process of verbalization itself transforms thought, drawing attention to some aspects of the environment and not others, solidifying meaning, and creating an observable artifact. Secondly, as an observable artifact, it can be reflected upon, questioned, manipulated, and restructured. And thirdly, internalization of the now differently understood externalized artifact may occur. (Swain 2006: 101)

Gleichzeitig oder nachträglich zur (sprachlichen) Handlung erhobene Verbalprotokolle stellen daher keine zuverlässigen Repräsentanten von Bedeutung dar, sondern werden als Mittel zur Bedeutungskonstruktion und als Hinweis auf 
Kognitionen sowie den Verursacher dieser Kognitionen wahrgenommen (Swain 2006: 100). Verbalprotokolle ermöglichen somit kein direktes Abbild von Kognitionen, sondern setzen diese bei gleichzeitiger Generierung neuer Denkprozesse frei. Daher messen Vertreter der Informationsverarbeitungstheorie und der SKP dem Einsatz der Datenerhebungsmethode eine gänzlich konträre Funktion bei. Das Forschungsziel der Ersteren liegt im Erstellen von Kognitionsmodellen, während Letztere beabsichtigen, damit Lernen als sozial, kulturgebunden und durch Sprechen vermittelt zu untersuchen: „Verbal protocols in this view are understood more as an opportunity for participants to become reflective about their cognitive processes by representing them in spoken language" (Deschambault 2011: 8).

Unter dieser Prämisse muss der Anspruch eines sorgfältig strukturierten Forschungsdesigns zur Validitäts- und Reliabilitätssteigerung beim Einsatz von LD und (V)LE und die Analyse der dadurch erhaltenen Daten einer erneuten Prüfung unterzogen werden, welche im folgenden Abschnitt erfolgt.

\section{Auswirkungen der soziokulturellen Perspektive auf die Erhebung und Analyse von LD- und (V)LE-Daten}

\subsection{Datenerhebung und deren Vorbereitung als Situierung}

Einsichten, die die SKP auf Verbalprotokolle gewährt, wirken sich auf die Rolle der Vorbereitung der Datenerhebung (s. Abschnitt 1) als auch auf die Erhebung selbst aus. Situiertheit und die damit verbundene Tendenz zur Adressierung stellt, wie unter Punkt 2 und 3 gezeigt, ein natürliches Phänomen im Rahmen eines jeden LD- und (V)LE-Erhebungssettings dar. Gleichzeitig sind hier die Grenzen eines vorgelagerten Trainings von LD und (V)LE und eines prototypisierten Untersuchungsaufbaus zu verorten. Die aufgeführten Elemente der Adressierung beim VLE belegen, dass sich die Wiederspiegelung der sozialen Situiertheit in den Daten trotz vorherigen Übens der Methode und einer weitgehenden Minimierung jeglicher Einflüsse auf den Denk- und Verbalisierungsprozess nicht vermeiden lässt: „The goal should not be so much to neutralize these effects but to assume that they exist and then to identify and account for them in the data collection and analysis" (Smagorinsky 1998: 169).

Damit erweist sich auch die durch die Instruktion anvisierte strikte Trennung in Datentyp 1, 2 und 3 als hinfällig, da Kognitionen immer einer sowohl externen als auch internen Beeinflussung unterliegen und damit bei ihrer Umformung in Sprache möglichen Änderungen oder situierenden Begleiterscheinungen ausgesetzt sind.

Die Potenziale der Datenerhebungsvorbereitung basieren vielmehr auf der Möglichkeit des Vertrautmachens mit den Rahmenbedingungen der Erhebung, 
mit der Aufgabenbearbeitungsprozedur und mit dem Vorgang des LD oder LE seitens der/des Forschenden und der/des Untersuchungsteilnehmenden. Hinzu kommt die Familiarisierung mit der (ein gewisses Mindestmaß an Medienkompetenz voraussetzenden) meist videobasierten Datenerhebungstechnik zur Optimierung der Erhebungsabläufe.

Des Weiteren verbinden Vertreter der SKP mit den Entscheidungen zum Prozedere der Datenerhebung die Vergegenwärtigung des Umgangs mit der Aufgabe seitens der Lernenden und mit der Erhebungssituation: „It would be important to understand how and why the participant constructs an interpretation of the situation in the way he or she does to understand why this particular account is rendered in this particular context" (Smagorisky 1998: 169). Dies gewährleistet zudem die Berücksichtigung einer Vielzahl an Faktoren, die auf die Erhebungssituation einwirken, wie bspw. des komplexen Beziehungsgefüges zwischen Forschender/m und Beforschter/m und eröffnet den Forschenden nicht nur die etische, sondern auch die emische (teilnehmerbezogene) Perspektive auf die Untersuchungsgegenstände (vgl. Markee/Kasper 2004). Den weiteren Auswirkungen eines solchen Umgangs mit LD- und (V)LE-Daten auf die Datenanalyse widmet sich der nun folgende Abschnitt.

\subsection{Potenziale für die Analyse von LD- und (V)LE-Daten}

Vor dem Hintergrund der bisherigen Ausführungen bleibt zu prüfen, welche Implikationen das soziokulturelle Verständnis auf LD und (V)LE für den Einsatz, die Auswertung und Interpretation von Verbalprotokollen bereithält.

Die bisher noch überschaubare Zahl von Studien, die sich auf soziokulturelle Vorannahmen stützen, verwenden die Erhebungsmethode vornehmlich für die Erforschung von Feedback (Adams 2003, Nabei 2002, Swain/Lapkin 2002, TocalliBeller/Swain 2005), Bedeutungserschließung beim Lesen (Deschambault 2011) oder Strategiennutzung (Holunga 1994). Studien, die sich des LDs bedienten, nehmen dabei eine weniger radikale Neuausrichtung der Funktion von Verbalprotokollen vor als Vertreter, die mit der retrospektiven Variante des (V)LE arbeiten.

Deschambaults Untersuchung von Bedeutungserschließungsprozessen beim Lesen $(2007,2011)$ mittels LD ist in diesem Zusammenhang besonders aufschlussreich, da er zeigen kann, dass die Datenneutranskription und -analyse unter SKP zu neuen Erkenntnissen über das Zustandekommen der Bedeutungserschließung führt. Wurde das Phänomen 2007 von ihm als interner kognitiver Vorgang beschrieben, der allein vom Untersuchungsteilnehmenden geleistet wurde, erkennt Deschambault 2011 bei der erneuten Datenauswertung, dass dabei ein kollaborativer Ko-Konstruktionsprozess zwischen Forscher und Probanden vorliegt, also Einsichten auf Lernendenseite auch durch die Interaktion (z. B. in Form von Rückfragen, steigender Intonation oder selbstinitiierter Selbstreparatur) 
mit dem Forschenden zustande kamen. Hier zeigt sich, dass die Anwesenheit des Forschenden und das Bewusstsein um den Untersuchungskontext zu einer Dialogizität und Adressiertheit in LD-Protokollen geführt haben, die einen wesentlichen Einfluss auf die im Forschungsinteresse stehenden Kognitionen ausübten.

In Untersuchungen, die sich des (V)LE bedienen, wird die Frage nach der Reaktivität, also der potenziellen Beeinflussung der kognitiven Prozesse durch das nachträgliche Verbalisieren der Gedanken neu gewichtet. Gilt es in Studien, die sich am Informationsverarbeitungsparadigma orientieren, mögliche Einflussfaktoren auf die Kognitionen durch ein vorstrukturiertes Datenerhebungsverfahren bestmöglich zu kontrollieren, wird unter soziokultureller Perspektive diese Reaktivität als nicht vermeidbar und dadurch als Teil des treatments sogar als erwünscht betrachtet (Swain 2006). Bemerkenswert erscheint hier, dass die von Swain geleiteten Studien sich dafür ausschließlich des (V)LEs bedienten, also zum Nachweis der positiven Reaktivität von Verbalprotokollen eine nachträgliche besser als eine parallele Verbalisierung geeignet $\mathrm{zu}$ sein scheint. Jüngste Resultate von Metaanalysen zur Reaktivität können dies für einige Fälle bestätigen (Bowles 2010). So gesehen erhält (V)LE in fremdsprachenlerntheoretischen Forschungsdesigns eine gänzlich andere Funktion, wie am Beispiel der folgenden Studien kurz verdeutlicht werden soll.

Swain/Lapkin (2002) setzten video stimulated recall ein, um Gedanken während einer noticing-Aktivität zu untersuchen, bei der Französischlernende zum Vergleich zwischen der Originalversion einer von ihnen selbst geschriebenen Geschichte und der durch Dritte überarbeiteten Variante des Textes aufgefordert wurden. Der Fokus lag dabei auf dem Bemerken von Unterschieden zwischen der eigenen und der reformulierten Textversion. Die von den Lernenden im Anschluss an das noticing verfasste Endversion des Textes legte die Vermutung der Forschenden nahe, dass neben dem durch den überarbeiteten Text bereitgestellten korrektiven Feedback durch Dritte auch die explizite Artikulation der Bedeutungsunterschiede zwischen ursprünglich beabsichtigter und durch die Korrektur abgewandelter Aussage des Textinhalts durch die Lernenden selbst zur Textüberarbeitung beitrugen. Kritisch merkt Swain (2006) an, dass hierbei nicht untersucht werden konnte, inwieweit jeweils Feedback und Verbalisierung zum Lerneffekt beigetragen hatten. Diesen Nachweis erbringt Adams (2003), indem sie bei einer identisch gearteten noticing-Studie die Posttestresultate von drei Gruppen (G1: nur Pre- und Posttest, G2: noticing + Tests, G3: noticing + stimulated recall + Tests) vergleicht und feststellt, dass für die Reformulierungen, die von den Lernenden während ihrer LE-Protokolle aufgegriffen wurden, bessere Testergebnisse erzielt wurden. Auch die Studie von Nabei (2002) verdeutlicht, dass nach einem Feedback durch recasts der Einsatz von VLE zur Verbesserung der Testresultate bezüglich solcher Items führte, zu denen die VersuchsteilnehmerInnen sich zusätzlich durch stimulated recall äußerten. 


\section{$5 \quad$ Zusammenfassung und Fazit}

Nach eingehender Analyse und Diskussion von Phänomenen der sozialen Situiertheit am Beispiel der Adressierung in VLE-Daten und der Begründung ihres Auftretens unter Zuhilfenahme der soziokulturellen Perspektive auf Verbalprotokolle bestärkt sich die Einsicht, dass rein kognitivistisch ausgerichtete, dem Rationalismus verhaftete Forschungsdesigns zur Untersuchung von Fremdsprachenlehr- und -lernprozessen, die sich der Erhebungsmethode des LD oder LE bedienen, bezüglich ihrer Angemessenheit zum Erfassen von Sprache und Denken an ihre Grenzen gestoßen sind. Hinsichtlich der Beschaffenheit von LD- und (V)LE-Daten bleibt festzuhalten, dass eine Unterscheidung in drei Datentypen aus SKP nicht haltbar ist, da die Elemente der sozialen Situiertheit, wie am Beispiel der Adressierung vorgeführt, ein diesen Daten inhärentes Charakteristikum darstellen. Soziokulturelle Faktoren sind Bestandteil und Einflussgröße einer jeden Kommunikations- und damit auch Erhebungssituation verbaler Daten. Daher gilt es diese Faktoren nicht auszublenden, sondern als natürliche Datengenerierungsbedingungen bewusstzumachen und zu reflektieren, um damit aus Erhebungskontext/-situation, erhebungsbegleitenden Interaktionen, sozialen Rollen, kulturellen und historischen Hintergründen wertvolle zusätzliche Einsichten für die Datenauswertung und -interpretation zu gewinnen:

A protocol should not be analyzed only for what it allows the researcher to infer about the individual mind, but should be analyzed as a situated practice with antecedent cultural history. [...] History can only be partially reconstructed, and so the cultural precedents for a protocol could only be sampled and inferred. (Smagorinsky 1998: 174)

Dieser Aushandlungsprozess zwischen emischer und etischer Perspektive in der Erforschung von Fremdsprachenlernen und -gebrauch gestattet es, kultur- und sprachspezifische Dimensionen an den Untersuchungsgegenstand heranzutragen, die neben den Forschenden auch den Untersuchten selbst die Benennung und das Verständnis von Phänomenen ermöglichen.

Im Hinblick auf die funktionale Ausrichtung der Forschungsdesigns bleibt zu betonen, dass sich kognitivistische und soziokulturelle Sichtweisen auf Verbalprotokolle je nach Erkenntnisinteresse nicht gänzlich ausschließen. Reaktivität kann demnach auch als erwünschter Faktor wahrgenommen werden (vgl. Bowles 2010), wobei sich bisher ausschließlich retrospektiv angelegte Erhebungsdesigns die positive Reaktivität für die Untersuchungsziele zunutze machten, bei denen eine nachträgliche Verbalisierung zu Bewusstmachungs- und damit Lernprozessen führt.

Im Rahmen der Fremdsprachenforschung erscheint die SKP auf Verbalprotokolle besonders für Studien zur Untersuchung von Interaktionen oder sozialen Lernprozessen (z. B. Feick in Vorbereitung, Fujii/Mackey 2009, Lam 2008, Schepens et al. 2006) in natürlichen Forschungskontexten vielversprechend. Hierbei ermöglicht eine transparente Darstellung von Erwartungen bzw. Voran- 
nahmen und Einflussfaktoren seitens der Forschenden und der Untersuchungsteilnehmenden bezüglich der Erhebungssituation eine kontextadäquate Einordnung und Auswertung der Daten.

\section{Literatur}

Adams, Rebecca (2003). L2 output, reformulation and noticing: Implications for IL development. Language Teaching Research 7: 347-376.

Bakhtin, M. Mikhail (1984): Problems of Dostoevsky's Poetics. (C. Emerson, Ed. \& Trans.) Minneapolis: University of Minnesota Press.

Bayer, Claus (1973): Verteilung und Funktion der sogenannten Parenthese in Texten gesprochener Sprache. Deutsche Sprache 1: 64-115.

Bloom, Benjamin S. (1954): The thought processes of students in discussion. In: French, Sydney J. (ed.): Accent on teaching: Experiments in general education. New York: Harper. 23-46.

Bowles, Melissa A. (2010). The think-aloud controversy in second language research. New York: Routledge.

Deschambault, Ryan (2007): A descriptive study of middle school ESL students 'reading moves and uses of visual inscriptions when inferring the meaning of unknown words in a science passage. Unpublished master's thesis, University of Victoria, British Columbia, Canada.

Deschambault, Ryan (2011). Thinking aloud as talking-in-interaction: reinterpreting how L2 lexical inferencing gets done. Language Learning 62/1: 266-301.

Dörnyei, Zoltán (2007). Research methods in applied linguistics. Oxford: University Press.

Ericsson, K. Anders / Simon, Herbert A. (1984): Protocol analysis: verbal reports as data. Cambridge u.a.: The MIT Press.

Ericsson, K. Anders / Simon, Herbert A. (1993). Protocol analysis: verbal reports as data. $2^{\text {nd }}$, revised ed. Cambridge: The MIT Press.

Ericsson, K. Anders / Simon, Herbert A. (1998). How to study thinking in everyday life: contrasting think-aloud protocols with descriptions and explanations of thinking. Mind, Culture and Activity 5/3: 178-186.

Fandrych, Christian / Graefen, Gabriele (2001). Text commenting devices in German and English academic articles. Multilingua 21/1: 17-43.

Feick, Diana (2012). Videobasiertes Lautes Erinnern als Instrument zur Untersuchung fremdsprachlicher Gruppenaushandlungsprozesse. In: Doff, Sabine (Hrsg.): Fremdsprachenunterricht empirisch erforschen. Grundlagen - Methoden - Anwendung. Tübingen: Narr. 202-217.

Feick, Diana (in Vorbereitung). Autonomes Lernen als Sozialer Prozess. Autonomiepotenziale gruppeninterner Aushandlungsprozesse am Beispiel von Handy-Videoprojekten im DaFUnterricht. (Arbeitstitel eines laufenden Dissertationsprojekts, Universität Leipzig.) 
Fujii, Akiko / Mackey, Alison (2009). Interactional feedback in learner-learner interactions in a task-based EFL classroom. IRAL: International Review of Applied Linguistics in Language Teaching 47/3-4: 267-301.

Gass, Susan M. / Mackey, Alison (2000). Stimulated recall methodology in second language research. Mahwah: Erlbaum.

Heine, Lena (2005). Lautes Denken als Forschungsinstrument in der Fremdsprachenforschung. Zeitschrift für Fremdsprachenforschung 16/2: 163-185.

Heine, Lena / Schramm, Karen (2007). Lautes Denken in der Fremdsprachenforschung: Eine Handreichung für die empirische Praxis. In: Vollmer, Helmut J. (Hrsg.): Synergieeffekte in der Fremdsprachenforschung. Frankfurt am Main: Lang. 167-206.

Henderson, Lyn / Tallman, Julie I. (2006). Stimulated recall and mental models: Tools for teaching and learning computer information literacy. Lanham: Scarecrow Press.

Holunga, Susanne (1994). The effect of metacognitive strategy training with verbalization on the oral accuracy of adult second language learners. Unpublished doctoral dissertation, University of Toronto (OISE), Toronto.

Knorr, Petra / Schramm, Karen (2012). Datenerhebung durch Lautes Denken und Lautes Erinnern in der fremdsprachendidaktischen Empirie. In: Doff, Sabine (Hrsg.): Fremdsprachenunterricht empirisch erforschen. Grundlagen - Methoden - Anwendung. Tübingen: Narr, 184-201.

Lam, Wendy Y. K. (2008). Metacognitive strategy use: accessing ESL learners' inner voices via stimulated recall. Innovation in Language Learning and Teaching 2/3: 207-223.

Leont'ev, Aleksei N. (1981). Problems in the development of the mind. Moscow: Progress Publishers.

Markee, Numa / Kasper, Gabriele (2004). Classroom talks: an introduction. The Modern Language Journal 88/4: 491-500.

Nabei, Toshiyo (2002). Recasts in classroom interaction: a teacher's intention, learners awareness and second language learning. Unpublished doctoral dissertation, University of Toronto (OISE).

Robinson, Mary A. (1992). Introspective methodology in interlanguage pragmatics research. In: Kasper, G. (ed.): Pragmatics of Japanese as a native and target language. Honolulu: University of Hawai'i Press. 27-82.

Sasaki, Tomomi (2008). Concurrent think-aloud protocol as a socially situated construct. IRAL: International Review of Applied Linguistics in Language Teaching 46/4: 349-374.

Schepens, Annemie / Aelterman, Antonia / van Keer, Hilde (2007). Studying learning processes of student teachers with stimulated recall interviews through changes in interactive cognitions. Teaching and Teacher Education 23/4: 457-472.

Schwitalla, Johannes (2001). Gesprochene-Sprache-Forschung und ihre Entwicklung zu einer Gesprächsanalyse. In: Brinker, Klaus / Antos, Gert / Heinemann, Wolfgang / Sager, Sven Frederik (Hrsg.): Text- und Gesprächslinguistik. Berlin: Walter de Gruyter. 896-903.

Smagorinsky, Peter (1998). Thinking and speech and protocol analysis. Mind, Culture, and Activity 5/3: 157-177. 
Smagorinsky, Peter (2001). Rethinking protocol analysis from a cultural perspective. Annual Review of Applied Linguistics 21: 233-245.

Swain, Merrill (2006). Verbal protocols: what does it mean for research to use speaking as a data collection tool? In: Chalhoub-Deville, Michelene / Chapelle, Carol A. / Duff, Patricia A. (eds.): Inference and generalizability in applied linguistics: Multiple perspectives. Amsterdam: John Benjamins. 97-113.

Swain, Merrill / Lapkin, Sharon (2002). Talking it through: two French immersion learners' response to reformulation. International Journal of Educational Research 37/3-4: 285-304.

Tocalli-Beller, Agustina / Swain, Merrill (2005). Reformulation: the cognitive conflict and L2 learning it generates. International Journal of Applied Linguistics 15/1: 5-28.

Wertsch, James V. (1981). The concept of activity in soviet psychology. In: Wertsch, James V. (ed.): The concept of activity in Soviet psychology. Armonk: Sharpe. 3-36.

Wygotski, Lev S. (1934, 2002). Denken und Sprechen. Weinheim: Beltz.

Witte, Stephen / Cherry, Roger (1994). Think-aloud protocols, protocol analysis, and research design: An exploration of the influence of writing tasks on writing processes. In: Smagorinsky, Peter (ed.): Speaking about writing. Thousand Oaks: Sage. 20-54. 


\section{Introspektive Verfahren in der Sprachtestforschung}

\section{Ulrike Arras}

Traditionally, research in language testing is conducted using quantitative methods, such as psychometrics and statistics. Since the 1980s, however, an increasing number of qualitative research methods, such as introspection and verbal protocols, have been implemented. This is based on the concern that quantitative methods are unable to discover and explain cognitive processes and factors which influence testing and assessment satisfactorily. By and large, two areas have since been investigated using introspective methods. Firstly, the field of test-taking strategies: Are the tests we develop able to elicit those language behaviours which we aim to measure? Secondly, the field of rating test products such as oral and written texts: What kind of strategies and cognitive processes can be observed in raters? On which aspects of the text do raters base their assessment and why do they do so? Thus, introspective methods in the context of language test quality have two benefits: We learn about how tests function and whether we can measure that which we sought to. Additionally, the findings might be used for our language behavior as learners and our assessment behaviour as teachers and raters. Both aspects are crucial for test validity. This article aims to give an overview of the state of the art in language testing with regard to introspective methods and discusses its potential and limitations.

\section{Begründungszusammenhang}

Tests und Sprachtests sind traditionell vor allem die Spielwiese quantitativer Forschungsmethoden. Die Reklamation von Testgütekriterien wie z. B. die Reliabilität, die mit Hilfe statistischer Verfahren kontrolliert werden kann, gehört entsprechend $\mathrm{zu}$ den Errungenschaften der Qualitätssicherung auf dem Gebiet der Sprachtestforschung. Einen Überblick über forschungsmethodologische Entwicklungen auf dem Gebiet des Sprachtestens der letzten Dekaden verdanken wir Banerjee/Luoma (1997), Bachman (2000) sowie Lumley/Brown (2005). Demnach ist die Sprachtestforschung bis in die 1970er Jahre geprägt von quantitativen Methoden.

Nichtsdestotrotz bleibt das Unbehagen, allein mit quantitativen Verfahren ein so zentrales Problem des Fremdsprachenunterrichts wie die Leistungsmessung zu erforschen. Daher sind Entwicklungen erfreulich, die sich mittlerweile in der Sprachtestforschung abzeichnen und die mit Hilfe qualitativer Methoden spezifische Strategien und Prozesse im Kontext Sprachtest sowie die Produkte von Leistungsmessung (d. h. schriftliche und mündliche Prüfungsleistungen) bzw. deren Merkmale zu erforschen suchen. Lumley/Brown (2005: 833) fassen diese Entwicklung in ihrem Überblick zu Methoden in der Sprachtestforschung folgendermaßen zusammen: 
There has been a move from positivistic research focusing on properties of tests and scores toward a broader and more critical examination of a wide range of validity issues embracing construct definition as well as language testing practice and policy.

Seit den 1990er Jahren lässt sich somit eine Hinwendung zur Erforschung von Faktoren beobachten, die die Performanz beeinflussen. Bachman (2000) zählt folgende Forschungsgebiete auf:

- Merkmale, die den Prozess des Testens determinieren, unter Einschluss von Aspekten wie der Bewertung von Prüfungsleistungen durch BeurteilerInnen;

- Prozesse und Strategien der Prüflinge bei der Bewältigung der Testaufgaben;

- Merkmale der Prüflinge selbst.

Der Einbezug von Faktoren wie etwa Persönlichkeitsmerkmalen der Prüflinge sowie impact-Faktoren in die Erforschung von Sprachtests macht qualitative Forschungsmethoden erforderlich. Hierbei bieten sich introspektive Verfahren an, die Einblicke in kognitive Prozesse erlauben. Exemplarisch genannt seien die auf introspektiven Daten bzw. verbal protocols beruhenden Arbeiten zur Analyse der test-taking processes bei Hörverstehenstests etwa von Buck (1991) oder jüngst von Rossa (2012), die Analysen der Beurteilungsprozesse und -strategien von Lumley (2005) und Arras (2007) zu schriftlichen Prüfungsleistungen sowie jüngst die Arbeiten von Ducasse (2010) und May (2011) zu den Prozessen bei mündlichen Prüfungen.

Interessant ist hierbei vor allem die Entwicklung, Daten außerhalb des eigentlichen Tests in die Interpretation einzubeziehen (Lumley/Brown 2005: 849). Denn damit stellen sich der Sprachtestforschung interdisziplinäre Fragen etwa aus angrenzenden Gebieten wie den Sozialwissenschaften und der Psychologie. Dies hängt auch mit dem veränderten bzw. erweiterten Validitätsbegriff (s. insb. Messick 1989) zusammen.

As in other areas of educational measurement, validity is no longer viewed as a set of measurable phenomena (based on traditional categories of construct, content, predictive and concurrent validities) but as an integrated and holistic judgment that is based on the collection of evidence from a number of different areas [...]. In this revised conceptuallization, the main concern is the validity of the inferences made about test-takers on the basis of their scores, that is, the meaning of scores. (Lumley/Brown 2005: 840f.)

Konsequenterweise geht es darum, sowohl das Produkt von Leistungsmessung, also konkrete schriftliche oder mündliche Leistungen, als auch den Leistungsprozess, etwa die kognitiven und strategischen Handlungen, die diesen Leistungen zugrunde liegen, zu verstehen. Und hierbei sind qualitative Daten erforderlich (ebd.). Folgende Entwicklungen sind erkennbar: 
- Mehr und mehr werden heute auch externe Merkmale in die Sprachtestanalyse einbezogen, indem etwa der soziale und (bildungs)politische Kontext sowie die Instrumentalisierung und der Impact von Sprachtests in den Fokus geraten (Shohamy 2001).

- Bei den qualitativen Methoden zur Erforschung von Sprachtests wird zum einen die Diskursanalyse eingesetzt, etwa zur Analyse mündlicher Leistungen (McNamara/Hill/May 2002). Zum anderen werden mittels Introspektion erhobene Daten, in erster Linie Lautes Denken in Form von verbal protocols, hinsichtlich kognitiver Prozesse analysiert. ${ }^{1}$

Einen Überblick zum Einsatz qualitativer Methoden in der Sprachtestforschung legen Banerjee und Luoma (1997) vor. Sie führen an, dass bereits in den 1990er Jahren zunehmend qualitative Ansätze in der Sprachtestforschung berücksichtigt werden. Die Arbeiten zeigen nach Bachman (2000: 7) zudem, dass verschiedene Ansätze Anwendung finden und miteinander kombiniert werden, etwa „expert judgments, introspective and restrospective verbal reports, observations, questionnaires and interviews, as well as text analysis, conversational analysis and discourse analysis."

Introspektive Verfahren im Kontext Sprachtestforschung konzentrieren sich im Wesentlichen auf zwei Problembereiche, erstens auf die Eruierung von Teststrategien seitens der Prüflinge und zweitens auf die Analyse der Strategien zur Beurteilung von Prüfungsleistungen seitens der BewerterInnen. Der erste Aspekt erfasst also den Umgang mit Testaufgaben, also die kognitiven Prozesse, die bei der Bearbeitung von Testaufgaben $\mathrm{zu}$ beobachten sind, der zweite die Interpretation der Ergebnisse, also die Beurteilung der Prüfungsleistungen. Beides sind zentrale Anliegen jedweden Testens, denn zum einen wird fokussiert, ob das, was man mit bestimmten Testaufgaben elizitieren will, etwa bestimmte Lesestrategien, diese auch tatsächlich hervorruft. Zum anderen wird betrachtet, wie die Produkte eines Tests, also z. B. ein schriftlich fixierter Text, der aufgrund einer bestimmten Aufgabenstellung verfasst wird, rezipiert und beurteilt werden. Beide Fragestellungen sollen im Folgenden genauer skizziert werden.

\section{Introspektion zur Erforschung von Teststrategien seitens der Prüflinge}

Die Leitfragen zur Erforschung von Teststrategien lauten etwa: Wie gehen Prüflinge mit Testaufgaben um? Wie lösen sie die Aufgaben, wie gehen sie konkret vor? Welche Strategien setzen sie ein? Und sind diese Strategien zielführend im Sinne des Testkonstrukts? Zugrunde gelegt ist dabei die Frage

\footnotetext{
${ }^{1}$ Nützliche Literatur hierzu s. insbesondere Ericsson/Simon (1993) sowie speziell für die Belange der Sprachtestforschung Green (1998).
} 
danach, ob die Aufgabe das elizitiert, was auch tatsächlich gemessen werden soll, ein nachgerade zentrales Anliegen von Sprachtests.

Ein Überblick über Studien auf diesem Gebiet stammt von Cohen (1998). Er fasst Untersuchungen zum Problem Teststrategien zusammen, die sich introspektiver Verfahren (verbal report techniques, qualitative methodologies, ebd.: 107) bedienen. Das entscheidende Moment sieht Cohen (ebd.: 92) in der Bewusstmachung. Eine Handlung kann demnach erst dann als Strategie bezeichnet werden, wenn sie bewusst ausgewählt wurde (s. auch Grotjahn 1997). Dies gilt gerade auch für Teststrategien:

Language use strategies are mental operations or processes that learners consciously select when accomplishing language tasks. These strategies also constitute test-taking strategies when they are applied to tasks in language tests. [...] Test-taking strategies will be viewed as those test-taking processes that the respondents have selected and of which they are conscious, at least to some degree. In other words, the notion of strategy implies an element of selection. Otherwise the processes would not be considered strategies. (Cohen 1998: 92, Hervorhebungen i. O.)

Während sich frühere Studien mittels introspektiver Verfahren vor allem dem Effekt von Itemtypen auf die Teststrategie widmen, ${ }^{2}$ konzentrieren sich jüngere Arbeiten auf spezifische Teststrategien je nach Fertigkeitsbereich. $\mathrm{Zu}$ nennen sind etwa Buck (1991) und Rossa (2012) zum Hörverstehen sowie Grupa (1999) zum Seh-Hörverstehen (zit. nach Grotjahn 2005). Cohen (1998) untersucht integrative Testaufgaben, die Lesen und Schreiben (summarizing), also kognitiv hoch komplexe Handlungen, erfassen. Ebenfalls zum Leseverstehen, aber anhand von Multiple Choice-Items liegt eine entsprechende Studie von Dollerup, Glahn und Hansen (1994) vor. Auch Wus (1998) Arbeit untersucht Teststrategien anhand von Multiple Choice-Items, allerdings zum Hörverstehen. Cohen/ Upton (2006) verwenden ebenfalls introspektive Verfahren (Laut-DenkenProtokolle) zur Überprüfung des revidierten Leseverstehenstests beim TOEFL. ${ }^{3}$

Zusammenfassend kann festgehalten werden, dass introspektive Verfahren Einblicke in die Funktionsweise von Aufgaben gewähren: Was löst ein bestimmtes Item-Format aus? Welche (beispielsweise Lese-)Strategie wird durch ein bestimmtes Aufgabenformat initiiert? Diese Einsicht erweist sich als zentral für die Sicherstellung der Testqualität. Denn nur wenn Aufgaben das elizitieren, was der Test vorgibt zu messen, ist der Test valide. Wird hingegen eine Aufgabe allem Anschein nach korrekt gelöst (d. h. entspricht die Lösung dem vorgegebenen Lösungsschlüssel), beruht die Lösung jedoch auf Strategien, die mit dem Test nicht beabsichtigt werden und die somit nicht Teil des Testkonstrukts

\footnotetext{
${ }^{2}$ Eine frühe empirische Arbeit auf diesem Gebiet verdanken wir Stemmer (1991) speziell zum Format C-Test.

3 Eine Übersicht über die Forschungsliteratur zu Teststrategien unter Verwendung von Verbaldaten s. Cohen/Upton (2006: 11).
} 
sind, so liegt eingeschränkte Validität vor. Bei bestimmten Aufgabenformaten, etwa geschlossenen Item-Formaten wie Multiple Choice, kann die Bewältigung der Aufgabe nicht direkt beobachtet werden, vielmehr liegt mit der Lösung lediglich ein Indiz vor. Wir schließen beispielsweise bei einer Leseverstehensaufgabe mit Multiple Choice-Aufgaben von der korrekten Lösung auf die angemessene Rezeption und Verarbeitung des Lesetextes, also auf die intendierte kognitive Operation. Welche konkreten Leseverstehensstrategien aber tatsächlich angewendet wurden oder ob der Text überhaupt gelesen wurde, können wir nicht mit Sicherheit sagen. Cohen (1998: 107) schlussfolgert in diesem Zusammenhang: „Verbal report can help us see what items are actually testing, aiding us in making decisions about which items to keep and which to throw out." Er schlägt somit vor, Verbaldaten in die Erprobung von Testaufgaben einzubeziehen, denn ,,if a respondent has legitimate reasons for marking an item wrong, then the item needs to be rewritten". ${ }^{4}$ Dem entgegen stehen allerdings sicherlich Praktikabilitätserwägungen. Denn die Erhebung introspektiver Daten bei der Erprobung neuer Testaufgaben stellt einen erheblichen Aufwand dar. Zumindest sind jedoch solche Verfahren bei der Konzipierung neuer Sprachtests oder neuer Testformate dringend anzuraten.

\section{Introspektion zur Erforschung von Bewertungsstrategien seitens der BeurteilerInnen sprachlicher Prüfungs- leistungen}

Gleichermaßen zentral für die Testvalidität ist die Frage danach, wie Prüfungsleistungen beurteilt werden. Welche Prozesse sind $\mathrm{zu}$ beobachten? Worauf achten die BeurteilerInnen? Wovon lassen sich die BeurteilerInnen bei ihren Wahrnehmungen und Urteilen leiten? Inwiefern beziehen sie bereitgestellte Beurteilungsinstrumente, etwa Bewertungskriterien, ein? Und welche subjektiven Theorien liegen den Entscheidungen zugrunde? Introspektion vermag auch hier Aufschluss zu geben, inwiefern die Beurteilung im Sinne des Testkonstrukts erfolgt. Erst wenn, etwa durch Bewusstmachung und Reflexion, klar wird, worauf die BeurteilerInnen ihr Urteil gründen, kann sichergestellt werden, dass jene Aspekte beurteilt werden, die mit der Sprachprüfung erfasst werden sollen. Eine schriftliche Deutschprüfung beispielsweise, die vorgibt, kommunikative Kompetenzen zu messen, muss sicherstellen, dass bei der Beurteilung das Augenmerk nicht allein auf grammatische Korrektheit gerichtet wird.

Auf dem genannten Gebiet entstanden in den letzten Jahren einige empirische Arbeiten, die mittels Introspektion versuchten, den Beurteilungsprozess und die

\footnotetext{
${ }^{4}$ S. exemplarisch die Verwendung introspektiver Daten zur Validitätssicherung des neuen TOEFL-Lesetests (d. h. der neue internetbasierte iBT-TOEFL) bei Cohen/Upton (2007) und Cohen/Upton (2006).
} 
Strategien bei der Beurteilung schriftlicher Prüfungsleistungen zu eruieren (Überblick bei Arras 2007: 121ff.). Zu nennen ist insbesondere die Studie von Lumley (2005), die auch meine eigene Arbeit (Arras 2007) beeinflusst hat. ${ }^{5}$ In beiden empirischen Studien werden die BeurteilerInnen schriftlicher Prüfungsleistungen in den Blick genommen. Lumleys Untersuchung liegt ein Englischtest für EinwanderInnen in Australien zugrunde, die Studie von Arras entstand im Kontext Test Deutsch als Fremdsprache (TestDaF), einer Sprachprüfung für den Zugang zu Hochschulen in Deutschland. Insgesamt zeigen solche introspektiven Untersuchungen, wie komplex das Beurteilungsverhalten tatsächlich ist. Etliche Strategien lassen sich differenzieren. Die Beurteilungsarbeit selbst erweist sich als stark prozesshaft, gesteuert sowohl durch die Beurteilungsinstrumente, etwa Bewertungskriterien, aber insbesondere auch durch Persönlichkeitsmerkmale und subjektive Theorien, individuelle Erfahrungen und Erwartungen an die Prüfungsleistungen. Dies wiederum wirft Fragen auf zu speziellem Schulungsbedarf für BewerterInnen (s. dazu genauer Abschnitt 6).

Mittlerweile sind auch Arbeiten entstanden, die die Beurteilung mündlicher Leistungen in der Fremdsprache mittels introspektiver Verfahren bzw. anhand von verbal protocols untersuchen. Dies ist methodisch ungleich schwieriger, denn das Untersuchungsdesign muss dem Problem Rechnung tragen, dass kaum gleichzeitig gehört und gesprochen werden kann, d. h. die mündliche Leistung muss auditiv wahrgenommen werden und die eigenen Gedanken und Emotionen sollen laut verbalisiert werden. Hierzu haben nun Ducasse (2010) sowie May (2011) empirische, auf verbalen Daten beruhende Arbeiten vorgelegt. Sie untersuchen, wie mündliche Leistungen beurteilt werden, und zwar anhand von Daten aus Prüfungssituationen, wie sie oft, auch im universitären Kontext, durchgeführt werden: die Paarprüfung in einer face-to-face-Situation. Im Gegensatz zu Einzelprüfungen, bei denen eine durchaus problematische kommunikative Situation vorliegt, die ein hierarchisches Gefälle zwischen PrüferIn und Prüfling aufweist, kommt in einer Paarprüfung hinzu, dass die beiden Prüflinge gehalten sind $\mathrm{zu}$ interagieren.

\section{Einige methodologische Überlegungen}

Introspektive Verfahren (Lautes Denken, Retrospektion) liefern qualitative verbale Daten, die Aufschluss geben sollen über kognitive Prozesse, Strategien, aber auch emotionale Aspekte, Einstellungen, Motivation, Befindlichkeiten etc. seitens der ProbandInnen (vgl. hierzu insb. Schnell in diesem Band). Die Stärke

\footnotetext{
${ }^{5}$ Eine der ersten Arbeiten auf diesem Gebiet stammt von Huot (1988, 1993); allerdings untersucht er die Beurteilung muttersprachlicher (Englisch-)Prüfungsleistungen. Die Übertragbarkeit auf den Kontext Beurteilung fremdsprachlicher Leistungen ist nicht unproblematisch, s. auch Arras (2007: 125).
} 
introspektiver Daten liegt gerade darin, Einblick zu gewähren in Befindlichkeiten und bewusstseinsfähige Daten, die strategischen Entscheidungen zugrunde liegen. Introspektive Verfahren ermöglichen diesen Zugriff. Sie bringen jedoch auch einige gravierende Probleme und Einschränkungen mit sich, die bei der Entwicklung des Forschungsdesigns sowie bei der Interpretation der Daten und nicht zuletzt bei der Generalisierbarkeit der Ergebnisse berücksichtigt werden müssen. ${ }^{6}$ Sie seien im Folgenden kurz skizziert:

Zum einen ist zu beachten, dass das Datenerhebungsverfahren die Daten beeinflusst: Wir müssen davon ausgehen, dass introspektive Verfahren spezifische Verhaltensweisen (Strategien, Prozesse) initiieren, die auf das Verfahren selbst zurückzuführen sind und damit den Verhaltensweisen unter realen Bedingungen nicht entsprechen. So erfordert beispielsweise die Beurteilung einer schriftlichen Prüfungsleistung unter Laut-Denk-Bedingungen das Vorlesen des Textes. Da diese interimsprachlichen Texte fehlerhaft sind, werden diese fehlerhaften Textstellen entsprechend verbalisiert. Vermutlich führt die doppelte Präsentation der sprachlichen Fehler (visuell über das Lesen und auditiv über das Hören) zu erheblicher Irritation, wenn nicht (wie in der Studie von Arras 2007 beobachtet) offensichtlich eine Vermeidungsstrategie eingesetzt wird: Die ProbandInnen verbalisieren fehlerhafte Textstellen korrigiert; $d$. h. sprachlich fehlerhafte Textstellen werden offensichtlich automatisiert korrigiert, es handelt sich sozusagen um ein „Sich-zurecht-Lesen“, vermutlich eine kooperative Verstehensstrategie den interimssprachlichen, teils stark fehlerhaften Texten gegenüber. ${ }^{7}$

Daneben stellt sich folgende Frage: Sind die Versuchspersonen überhaupt in der Lage zu verbalisieren, was mental präsent ist? Prinzipiell ist zu berücksichtigen, dass Persönlichkeitsfaktoren, etwa Extroversion vs. Introversion, akute Stimmungen, Erfahrung, Motivation, vor allem auch das Ausmaß an Bewusstheit für das eigene Handeln sowie die Fähigkeit zur Selbstreflexion etc. die Qualität und auch den Umfang des Datenmaterials bestimmen. ${ }^{8}$

\footnotetext{
${ }^{6}$ Hilfreich in diesem Zusammenhang sind vor allem Greens (1998) kritische Einschätzungen introspektiver Verfahren bei der Erforschung von Beurteilungsstrategien (s. insb. Lumley 2005: 67ff. sowie Arras 2007: 140ff.). Rossa (2012) schlägt in einem ähnlichen Zusammenhang (Rekonstruktion von Teststrategien in Laut-Denken-Versuchssituationen) den Begriff ,Konfabulation' vor, ein aus der Medizin bzw. Psychologie entlehnter Begriff, der ein Verhalten beschreibt, bei dem Informationen verbalisiert werden, die - bewusst oder unbewusst nicht der Realität entsprechen, mit dem Ziel, gegenüber einer anderen Person oder auch sich selbst gegenüber Gedächtnislücken zu kaschieren bzw. antizipierten Verhaltenserwartungen zu entsprechen.

${ }^{7}$ Ausführlich hierzu Arras (2007: 270ff. und 469ff.). S. auch Lumley (2005: 283ff.).

${ }^{8}$ Eine Klassifizierung von Lernertypen in diesem Kontext nimmt Grotjahn (2007) vor. $\mathrm{Zu}$ Persönlichkeitsfaktoren bei BeurteilerInnen s. Arras (2007: 445ff. und 467ff.).
} 
Zudem stellen das bei Ericsson/Simon (1993) diskutierte Phänomen der heeded information oder heeded thoughts sowie der latente Erinnerungsfehler ein Problem dar. So sind verbalisierte erinnerte Informationen nicht zwangsläufig zuverlässig, denn die Erinnerung kann trügen. Die Autoren gehen von error in recall from LTM (long term memory) aus (Ericsson/Simon 1993: 258).

Dieses Phänomen ist besonders kritisch, wenn die ProbandInnen nicht in ihrer Erstsprache verbalisieren, beispielsweise wenn man eine mehrsprachige internationale LernerInnen-Gruppe hinsichtlich ihrer Test- oder Lernstrategien untersucht. Grundsätzlich sind Verbaldaten in der Erstsprache zu erheben, um zu verhindern, dass Informationen (Überlegungen, Befindlichkeiten etc.) nicht verbalisiert werden, allein weil sie nicht in der Zielsprache formuliert werden können und um den ohnehin problematischen Umstand zu relativieren, dass die Verbalisierung selbst Schwierigkeiten verursacht. Für die ProbandInnen sollten die Bedingungen der Untersuchungsdurchführung, also auch die Verbalisierung selbst, so einfach wie möglich gestaltet werden.

Dieser Punkt wirft Fragen bei Untersuchungen heterogener Gruppen auf, etwa der Erfassung von test-taking strategies in Gruppen von Prüflingen unterschiedlicher Herkunft, wie sie oftmals in Kursverbänden, auch zur Vorbereitung einer Sprachprüfung, zusammengesetzt sind. Wenn die ProbandInnen in ihrer Erstsprache introspektive Daten liefern, dann sollte sichergestellt werden, dass die Auswertung von native speakers vorgenommen wird. Oder aber die Versuchsgruppe sollte hinsichtlich der Herkunftssprache homogen organisiert sein, etwa indem nur eine bestimmte Zielgruppe untersucht wird, z. B. nur chinesische Lernende oder nur Lernende mit Französisch als Ausgangssprache.

Problematisch ist darüber hinaus die Frage nach der antizipierten sozialen Erwünschtheit. Das bedeutet, inwiefern filtern die Versuchspersonen bei der Introspektion, so dass nur solche Informationen preisgegeben, also verbalisiert werden, die als erwünscht oder seitens der Forscherin/des Forschers als erwartet antizipiert werden? Inwiefern möchten die ProbandInnen damit auch einem bestimmten Bild entsprechen, insbesondere wenn ein soziales Gefälle oder gar eine Abhängigkeit vorliegt (etwa Lernende gegenüber ihren forschenden LehrerInnen)? Diese Fragen beeinträchtigen direkt die Validität der Daten. Das bedeutet, introspektiv ermittelte qualitative Daten sind ggf. nur eingeschränkt valide. (Diskussion zur Nutzung von verbal protocol analysis im Kontext Sprachtestforschung insbesondere bei Green 1998).

Ein weiteres Problem bei der Verwendung von Verbaldaten (introspektive Daten, Lautdenkenprotokolle) ist die Repräsentanz von Informationen im Gedächtnis. Wird beispielsweise untersucht, wie BeurteilerInnen mündliche Leistungen beurteilen, so sind wir auf retrospektive Daten angewiesen, denn die mündlichen Leistungen müssen zunächst gehört (im Falle von Videomitschnitt auch visuell aufgenommen) werden und können erst retrospektiv, etwa nach Ablauf 
der Prüfungssequenz, von BeurteilerInnen kommentiert werden (methodische Diskussion dieses Sachverhalts s. bei Ericsson/Simon 1987, Green 1998, s. auch May 2011: 51f.).

Auf alle Fälle erscheint es notwendig, ProbandInnen gut in die Erhebungsweise introspektiver Daten einzuführen. Sie sollten Gelegenheit erhalten, das Verfahren zuvor zu üben. Doch auch ein vorhergehendes Training garantiert keine zuverlässigen Daten. ${ }^{9}$

Aufwendig bei introspektiven Daten ist nicht allein deren Erhebung, sondern vor allem auch ihre Auswertung. Meist werden alle Daten transkribiert, wobei sich die Komplexität der Transkription an der Fragestellung orientiert. Gleiches gilt für die Aufbereitung der Daten etwa mittels Segmentierung, Kodierung etc. ${ }^{10}$

Darüber hinaus sei auf methodologische Unterschiede aufmerksam gemacht, etwa die Differenzierung von introspektiven und retrospektiven Daten. ${ }^{11}$ Arras (2007: 143ff.) unterscheidet zudem selbstinitiierte von fremdinitiierter Retrospektion. So sind im Rahmen von Lautdenkprotokollen selbstinitiierte retrospektive Daten zu beobachten, bei denen die Äußerungen der Versuchspersonen ohne äußeren Stimulus erfolgen. Als Beispiel sei eine Stelle aus einem Protokoll angeführt, an der die Versuchsperson Schwierigkeiten bei der Einschätzung eines Aspekts der Aufgabenumsetzung erläutert, indem sie Bezug nimmt auf eine zuvor beurteilte Leistung: „Also für mich ist es immer so ein bisschen schwierig, wie begründen die jetzt ihre selektive Auswahl, ne? Also das hatte ich vorhin schon mal bei einem Kandidaten, ich weiß nicht mehr, welche Nummer das war, 3 oder 4“ (Arras 2007: 144). Hingegen liegen fremdinitiierte Daten vor, wenn die Versuchspersonen etwa im Anschluss an die Beurteilungsarbeit bzw. die Bearbeitung einer Testaufgabe aufgefordert wird, ihre Handlung zu kommentieren.

\section{Diskussion und Perspektiven für die weitere Forschung}

Trotz der skizzierten Einschränkungen sollte auf introspektive Verfahren in der Sprachtestforschung nicht verzichtet werden. Im Folgenden seien deshalb einige Überlegungen und Forschungsfragen angeführt.

- Inwiefern lassen sich introspektive Verfahren durch neue Technologien optimieren bzw. ergänzen? Können etwa bildgebende Verfahren die Interpretation der verbalen Daten unterstützen? Wie kann beispielsweise die Beobachtung von Augenbewegungen die Erfassung der kognitiven Prozesse bei der Bearbeitung eines Leseverstehenstests unterstützen? Oder können Video-

\footnotetext{
${ }^{9}$ Diskussion hierzu insb. bei Ericsson/Simon (1993), s. auch Lumley (2005).

${ }^{10}$ Praktisch orientierte Ausführungen hierzu s. insb. Green (1998).

${ }^{11}$ Hinweise hierzu etwa bei Banerjee/Luoma (1997: 277) sowie bei Cohen (1987).
} 
und Audioaufnahmen von mündlichen Prüfungssituationen die Beurteilung retrospektiv genauer nachvollziehbar machen?

- Inwiefern lassen sich introspektive Verfahren systematisch in die Qualitätssicherung von Sprachtests einbeziehen, etwa bei der Erprobung neuer Testaufgaben, wie Cohen (1998) vorschlägt, oder wenigstens bei der Konzipierung neuer Sprachtests und neuer Testformate, wie oben bereits angedeutet? Welche Praktikabilitätserwägungen limitieren dieses Vorgehen?

- Inwiefern lassen sich introspektive Verfahren zu Schulungszwecken oder im Rahmen von Aktionsforschung nutzen? Denn die Verfahren haben über das eigentliche Anliegen, qualitative Daten zu wissenschaftlichen Zwecken zu erheben, hinaus Nutzen, nämlich als Initiation für Reflexion. Hierbei eröffnen sich die in den folgenden beiden Unterkapiteln beleuchteten Perspektiven.

\subsection{Reflexion von Teststrategien}

Introspektion/Lautes Denken wird eingesetzt zur Initiation von Reflexion eigener Teststrategien (oder allgemeiner Sprachstrategien, etwa Lesestrategien, Hörverstehensstrategien, Strategien zur Organisation und Realisierung eines Redebeitrags oder eines schriftlichen Textes) mit dem Ziel, hilfreiche Strategien $\mathrm{zu}$ identifizieren und zu trainieren bzw. weniger hilfreiche Strategien zu verwerfen oder zu optimieren. Wie also lassen sich introspektive Verfahren bei Prüflingen bzw. Lernenden nutzen, um eine Reflexion der eigenen Teststrategien (etwa im Rahmen der Vorbereitung auf eine Sprachprüfung) in Gang zu setzen? Zugrunde liegt die Annahme, dass die Externalisierung innerer Rede mittels Introspektion/Retrospektion den Grad an Metakognition erhöht und damit Reflexionsfähigkeit initiiert wird, was wiederum zur Optimierung von Teststrategien führen soll. Hierbei kann einerseits differenziert werden zwischen spezifischen Lösungsstrategien und -techniken, die für die Bewältigung einer Aufgabe im Rahmen einer Testsituation eingesetzt werden, und allgemeinen sprachverarbeitenden Strategien und Techniken andererseits. So sind beispielsweise bestimmte Techniken im Umgang mit (fremdsprachlichen) Lesetexten, etwa die Visualisierung bzw. das Markieren von Schlüsselwörtern, das Exzerpieren und Paraphrasieren zentraler Aussagen eines Textes, relevant für die Bearbeitung eines Leseverstehenstests, aber ebenso relevant für das Lesen und die Verarbeitung von Literatur allgemein, also Teil allgemeiner Lesestrategien. Für den Lernprozess bedeutet dies wiederum, dass im Unterricht erworbene und bewusstgemachte Textverarbeitungsstrategien ebenso für die Anforderungen in einer Testsituation nutzbar sind. Und umgekehrt: Die in einer Testsituation nützlichen Strategien und Techniken können u. U. auch für die Text- bzw. Sprachverarbeitung außerhalb einer Testsituation dienlich sein (s. auch den Begriff strategic competence bei Bachman/Palmer 1996). Der Unterschied liegt 
im Wesentlichen in der Aufmerksamkeitssteuerung. Sie wird im Falle eines Leseverstehenstests gemeinhin durch Items geregelt: Die Informationssuche im Lesetext erfolgt dann anhand des im Item formulierten Problems, etwa wenn ein Multiple Choice-Item eine bestimmte Detailinformation aus dem Text fokussiert. Ähnlich verhält es sich mit Lernstrategien, die für die Lösung von Testaufgaben nutzbar gemacht werden können bzw. auf Testsituationen übertragen werden. So können im Unterricht eingesetzte Übungen zur Erweiterung des Wortschatzes (Sammeln von Synonymen, Paraphrasierung von Texten und Textpassagen) auch für die Bearbeitung von Leseverstehenstests dienlich sein. Dies zeigt sich auch in diversen Übungsmaterialien zur Testvorbereitung, die systematisch Synonyme, Umformulierung, Paraphrasierung etc. beispielsweise zur Prüfungsvorbereitung Leseverstehen einsetzen. ${ }^{12}$

Als reine test-taking strategies hingegen können solche Strategien (oder auch Techniken) bezeichnet werden, die dem Zeitmanagement oder der Selbstorganisation dienen. Als Beispiel soll eine Technik angeführt werden, die gelegentlich in Testvorbereitungsmaterial genannt wird, nämlich solche Items visuell markieren, die bereits beantwortet bzw. gelöst sind (etwa durchstreichen) oder die später noch einmal überprüft werden müssen (etwa mit einem Fragezeichen versehen), mit dem Ziel, den Überblick zu bewahren und zeitökonomisch vorzugehen (Lodewick 2010: 45f.).

Was den Einsatz introspektiver Daten zu Lernzwecken anbelangt, so ist mittlerweile auch gefordert worden, Introspektion einzusetzen, um Lernenden ihre eigenen Lernstrategien bewusst zu machen und dadurch ggf. eine Optimierung der Lernstrategien zu initiieren. Hierzu führt Beyer (2005) einige Studien aus dem Kontext Spracherwerb an, die den Einsatz von Introspektion als positiv für den Lernprozess bzw. für die Entwicklung und Bewusstmachung von Lernstrategien nachweisen. ${ }^{13}$ Den gewinnbringenden Effekt von Introspektion im Fremdsprachenunterricht fasst sie folgendermaßen zusammen:

- „Erhebung und Thematisierung von Aufgabenlösungsstrategien;

- Aufmerksamkeitsrichtung und Bewusstmachung gezielter fremdsprachlicher Strukturen;

- Erhebung von metasprachlichem Lernerwissen;

- Validierung von Tests;

- Erstellung von Lernerprofilen zur besseren Orientierung des Unterrichts an den individuellen Lernerbedürfnissen“ (Beyer 2005: 20)

${ }^{12}$ Exemplarisch für den Subtest Leseverstehen der Prüfung Test Deutsch als Fremdsprache (TestDaF) s. Roche (2005: 42ff.), Kniffka/Gutzat (2003: 19, 23), Lodewick (2010: 68f.), ebenso die „Hinweise und Tipps“ zur Prüfungsvorbereitung auf den Internetseiten des TestDaF-Instituts: http://www.testdaf.de/teilnehmer/tn-vorbereitung_tipp.php.

${ }^{13}$ Zur Relevanz der Bewusstmachung individueller Lernstrategien für den Lernprozess s. auch die Beiträge in Rampillon/Zimmermann (1997). 
Insbesondere für den Kontext Testen ermöglichen uns Introspektion bzw. Selbstreport herauszufinden, welche Teststrategien ungeeignet sind, etwa weil der Prüfling falsche Annahmen macht in Bezug auf das, was von ihm gefordert ist und wie seine Leistungen schließlich beurteilt werden. So berichten etwa Kleppin/Reich (2009: 96) von der test-taking strategy eines arabischen Studenten, der bei einem Hörverstehenstest, bei dem gefordert ist, Kurzantworten zu notieren, bestrebt ist, als Antwort möglichst alles aufzuschreiben, was er hört. Denn er geht davon aus, dass der Beurteiler bzw. die Beurteilerin sich die korrekte Antwort aus dem Material aussucht, das er als Antwort notiert: „Wenn ich viel schreibe, dann gibt es kein Problem. Der Prüfer wird die zusätzlichen Antworten löschen“ (,löschen“ meint hier vermutlich ,nicht berücksichtigen“. Und „,zusätzlich“ bedeutet sicher überflüssig im Sinne der Fragestellung). Der Prüfling geht also davon aus, dass sich die BeurteilerInnen sozusagen die richtige Antwort aus den Aufzeichnungen zusammensuchen. Erst die Selbstbeobachtung und Bewusstmachung sowie schließlich die Verständigung über die Testziele, das Anliegen der Sprachprüfung und schließlich auch über das entsprechende Vorgehen bei der Leistungsbewertung vermögen dem besagten Lerner zu verdeutlichen, dass diese Strategie keinen Nachweis über die zu messende Sprachkompetenz erbringt und somit auch in einem validen Test sinnlos ist.

\subsection{Reflexion von Beurteilungsstrategien}

Introspektion/Verbalisierung der inner speech bzw. Lautes Denken bietet sich auch für die Schulung von BeurteilerInnen (fremd-)sprachlicher (Prüfungs-) Leistungen an. Denn in einer empirischen Studie zum Beurteilungsverhalten konnte festgestellt werden, dass die Versuchspersonen (es handelte sich um erfahrene Beurteilerinnen, die wiederholt geschult und mit dem Beurteilungsinstrumentarium bestens vertraut waren) durch die Methode des Lauten Denkens angeregt wurden, ihr eigenes Beurteilungsverhalten zu reflektieren, zu hinterfragen, zu begründen. Dieses Verhalten, das für die Validität ihrer Urteile erforderlich ist, erfolgt unter normalen Arbeitsbedingungen, also das Beurteilen ,für sich' ohne Verbalisierung und ohne die als sozial erwünscht wahrgenommene ,Rechtfertigung' der Urteile bzw. der Urteilsschritte, vermutlich nicht (s. hierzu die Ausführungen und Vorschläge in Arras 2007: 462f.). Auch hierbei ist jedoch zu berücksichtigen, dass introspektive Verfahren sehr aufwendig sind, so dass sie sich für reguläre Schulungen ggf. als nicht praktikabel erweisen. Allerdings können diese Verfahren als Anregung bzw. als Projekt zur Integration in Schulungskonzepte dienen: 
- Schulung von BeurteilerInnen im Kontext von Sprachprüfungen, sowohl standardisiert als auch weitgehend nicht standardisiert mit dem Ziel der Kalibrierung, d. h. um auf der Basis von Bewusstmachung einheitliche Beurteilungsmaßstäbe innerhalb der Gruppe der BeurteilerInnen zu erzielen, wobei diese Maßstäbe in Einklang mit dem Testkonstrukt stehen müssen.

- Aus- und Weiterbildung von Fremdsprachen-Lehrkräften, also Einbindung introspektiver Verfahren in grundständige Studiengänge, in Lehramtsstudiengänge, in Aufbaustudiengänge mit dem Ziel, typische Verzerrungen bei der Beurteilung, etwa Zentraltendenz oder Positionseffekt, bewusst zu machen.

\section{Introspektive Verfahren im Kontext Schulung und Training}

Wie also können introspektive Verfahren über reine Forschungszwecke hinaus auch in Schulungen eingesetzt werden? Im Folgenden sollen praktische Überlegungen zu möglichen Szenarien im Kontext Schulung skizziert werden:

- In Schulungen von Lehrkräften bzw. BeurteilerInnen sollen schriftliche oder mündliche (Prüfungs-) Leistungen alleine oder in der Arbeitsgruppe beurteilt werden, dabei sollen die Beurteilungsstrategien und möglicherweise subjektiv geprägten Maßstäbe reflektiert werden.

- Beim Training von Lernenden, die sich auf eine Sprachprüfung vorbereiten, sind für die anstehende Prüfung relevante Testaufgaben alleine oder in der Arbeitsgruppe zu bearbeiten bzw. zu lösen. Dabei sollen unterschiedliche Vorgehensweisen wahrgenommen und kritisch überprüft werden. ${ }^{14}$

Introspektive Verfahren im Rahmen von Schulungen können als Paararbeit oder als Arbeit in der Kleingruppe in folgenden Szenarien konzipiert werden: ${ }^{15}$ Eine Person im Paar oder in der Kleingruppe löst die entsprechende Aufgabe unter Lautdenk-Bedingungen. Die Verbaldaten werden aufgezeichnet (Video- und/ oder Audioaufnahme). Die PartnerInnen hören zu, ohne das Wort zu ergreifen oder Rückfragen zu stellen, sie machen sich aber ggf. Notizen zu Vorgehen, Prozesshaftigkeit, Problemen, Widersprüchen etc. (am besten in einem dazu entwickelten Arbeitsblatt). Sodann werden die aufgezeichneten Verbaldaten gemeinsam gehört und reflektiert. Es besteht die Möglichkeit zu Rückfragen, zu retrospektiver Begründung bzw. Rechtfertigung für bestimmte Strategien, Arbeitsschritte, Entscheidungen. Am Ende sollte ein schriftlich (ggf. anhand eines Arbeitsblattes) fixiertes Arbeitsergebnis stehen, das gemeinsam im Paar oder in der Kleingruppe erarbeitet wird. Folgende Punkte können dabei relevant sein:

\footnotetext{
${ }^{14}$ S. auch die Vorschläge zu Reflexion und peer-Beobachtung bei Kleppin/Reich (2009: 109f.).

$15 \mathrm{~S}$. auch das in einer Pilotstudie verwendete Verfahren, dokumentiert in Arras/Marks/ Zimmermann (2009).
} 
- Welche Schwierigkeiten traten bei der Lösung der Aufgabe oder bei der Observation auf?

- Worauf gründen bestimmte Strategien? Etwa: Warum wurde eine Testaufgabe auf eine bestimmte Weise gelöst? Warum wurde eine bestimmte sprachliche Leistung so und nicht anders beurteilt? Welche Annahmen und subjektiven Theorien liegen zugrunde? Worauf sind diese zurückzuführen? (s. auch Arras 2009b).

- Wie haben sich die Beteiligten während der verschiedenen Phasen (Lösen der Aufgabe, Besprechung im Paar/in der Kleingruppe) gefühlt?

\section{Schlussfolgerungen}

Um die Validität eines Sprachtests sicherzustellen, ist es empfehlenswert, Daten zu erheben, die nachvollziehbar machen, wie die Prüflinge die Aufgaben bewältigen und die BeurteilerInnen schließlich Leistungen bewerten. Denn erst wenn die Testaufgaben die erwünschten kognitiven Prozesse, etwa bestimmte Verstehensstrategien bei einem Lese- oder einem Hörverstehenstest elizitieren, und BeurteilerInnen Prüfungsleistungen in Bezug auf das Testziel einschätzen, kann von einer validen Testaufgabe im Sinne des zugrundeliegenden Testkonstrukts ausgegangen werden. Die Beantwortung der folgenden Fragen trägt daher wesentlich zur Konstruktvalidität bei:

Inwiefern korrespondieren die von den Prüflingen in der Auseinandersetzung mit der Testaufgabe aktivierten mentalen Prozesse und Strategien mit den im Testkonstrukt theoretisch angenommenen mentalen Operationen und Teilkompetenzen? Und inwiefern führen unterschiedlich ausgeprägte Prozesse zu unterschiedlichen Testleistungen, erkennbar in unterschiedlichen Messergebnissen (s. Rossa 2012)? Schließlich: Inwiefern spiegeln die Beurteilungsstrategien und kriterien sowie die schlussendlichen Einschätzungen der Testleistung seitens der BeurteilerInnen das Testkonstrukt wider?

Um nun Einblicke in die genannten Prozesse zu gewinnen, erscheint Introspektion sinnvoll, denn sie bildet die kognitiven Prozesse seitens des Prüflings bei der Bewältigung von Testaufgaben und seitens der Beurteilerin oder des Beurteilers bei der Bewertungsarbeit ab. Trotz des nicht unerheblichen Aufwands, den introspektive Forschungsmethoden mit sich bringen, und der nach wie vor begrenzten Aussagekraft dieser Daten sollten entsprechende Begleituntersuchungen Bestandteil der Qualitätssicherung eines Sprachtests sein. Darüber hinaus geben introspektive Daten ggf. Aufschluss über erfolgreiche und weniger erfolgreiche Teststrategien, was wiederum für didaktische und curriculare Zwecke sowie für Beurteilungstrainings verwendet werden kann. 
Introspektive Verfahren erlauben also Einblicke, die über das primäre Anliegen eines Sprachtests hinausgehen: Wir erfahren, wie Sprache verarbeitet wird, welche Strategien dabei nützlich oder aber weniger nützlich sind, was wiederum didaktische Konsequenzen nach sich ziehen kann. $\mathrm{Zu}$ unterscheiden ist hierbei zwischen Teststrategien, die dem Testkonstrukt angemessen sind, und jenen, die lediglich zu einem korrekten Testergebnis führen, jedoch den intendierten kognitiven Prozessen und Verstehensstrategien nicht entsprechen. So sollten in einem Leseverstehenstest, der vorgibt, akademische Sprachverwendung zu messen, tatsächlich adäquate Lesestrategien zu beobachten sein, etwa kognitive Fähigkeiten, wie Orientierung im Text, Ausnutzung von Schlüsselwörtern etc. Der Einsatz von - evtl. sogar erfolgreichen, weil zum korrekten Testergebnis führenden - test wiseness tricks (Cohen/Upton 2006: 117) untergräbt hingegen die Validität eines Sprachtests. So führt etwa lucky guessing, beispielsweise ein zufällig korrekt gelöstes multiple-choice-Item, zwar zu einem guten Testergebnis, der Nachweis über die diesem Item zugrundeliegende Fähigkeit ist jedoch nicht erbracht worden.

Ähnlich verhält es sich mit der Frage nach der validen Beurteilung von Prüfungsleistungen. Introspektive Daten erlauben Einblick in die Strategien, Prozesse und begründeten Einschätzungen sprachlicher Leistungen. Die Bewusstmachung und Reflexion der subjektiv geprägten Beurteilungsfaktoren scheint nicht zuletzt für die Professionalisierung der BeurteilerInnen zentral, denn dies bildet die Grundlage für eine begründete Veränderung und Optimierung des Beurteilungsverhaltens, was wiederum von zentraler Bedeutung für die Testqualität ist.

Grundsätzlich sollte unser Ziel sein, zu Selbstreflexion zu animieren. Die Ausbildung von Reflexionsfähigkeit wird gemeinhin als grundlegende Fähigkeit betrachtet, um selbstständig zu lernen, zu arbeiten, Handlungen und Strategien $\mathrm{zu}$ überprüfen und begründet zu verwerfen oder beizubehalten. ${ }^{16}$ Dies gilt sowohl für Lernende und potenzielle Prüflinge als auch (und insbesondere!) für Lehrende und somit potenzielle BeurteilerInnen sprachlicher Leistungen.

Insgesamt betrachtet nehmen introspektive Verfahren heute einen wichtigen Platz in der Sprachtestforschung ein, insbesondere bei der Erforschung der Validität von Testaufgaben und Beurteilungsverfahren. Sie ergänzen quantitativ erhobene psychometrische Daten. Damit kann das von Bachman formulierte Plädoyer, ,to utilize quantitative and qualitative approaches in a complementary fashion", (Bachman 2000: 7) für eine ganzheitliche Sicht auf Sprachtests und ein besseres Verständnis der damit verbundenen Prozesse realisiert werden.

\footnotetext{
${ }^{16}$ Für den Bereich Fremdsprachenlernen s. die Beiträge in Rampillon/Zimmermann (1997), dort insbesondere Grotjahn. Für die Aus- und Weiterbildung s. Konzepte wie die Aktionsforschung (exemplarisch Altrichter/Posch 1998 sowie Reason/Bradbury 2002).
} 
Für beide Bereiche - Erforschung der Beurteilungsstrategien und Erforschung von test-taking strategies - gilt, dass noch etliche Aspekte einer genaueren Untersuchung und Systematisierung bedürfen, nicht zuletzt, um Konsequenzen für die Praxis abzuleiten, sei es für Unterricht, für Aus- und Weiterbildung von Lehrkräften oder für die Testkonzeption. Der vorliegende Beitrag hat versucht hierzu Anregungen zu geben. ${ }^{17}$

\section{Literatur}

Altrichter, Herbert / Posch, Peter (1998). Lehrer erforschen ihren Unterricht: Eine Einführung in die Methoden der Aktionsforschung. Bad Heilbrunn: Klinkhardt. 3. durchgesehene und erweiterte Auflage.

Arras, Ulrike (2007). Wie beurteilen wir Leistung in der Fremdsprache? Strategien und Prozesse bei der Beurteilung schriftlicher Leistungen in der Fremdsprache am Beispiel Test Deutsch als Fremdsprache (TestDaF). Tübingen: Narr.

Arras, Ulrike (2009a). What's on a rater's mind? Die Erforschung von Beurteilungsstrategien und ihre Bewusstmachung durch Schulungsmaßnahmen als Voraussetzungen für die Testvalidität. Zeitschrift für Angewandte Linguistik (ZfAL) 50: 33-45.

Arras, Ulrike (2009b). Subjektive Theorien als Faktor bei der Beurteilung von Prüfungsleistungen. In: Berndt, Annette / Kleppin, Karin (Hrsg.): Sprachlehrforschung: Theorie und Empirie: Festschrift für Rüdiger Grotjahn. Frankfurt am Main: Peter Lang. 169-179.

Arras, Ulrike / Marks, Daniela / Zimmermann, Sonja (2009). BeurteilerInnen in den Kopf geschaut. Wie das Verfahren des Lauten Denkens im Rahmen von Beurteilungsschulungen eingesetzt werden kann. DaF-Brücke. Zeitschrift für Deutschlehrerinnen und Deutschlehrer Lateinamerikas 11: 5-9.

Bachman, Lyle F. (2000). Modern language testing at the turn of the century: Assuring that what we count counts. Language Testing 17: 1-42.

Bachman, Lyle F. / Palmer, Adrian S. (1996). Language testing in practice. Oxford: Oxford University Press.

Banerjee, Jayanti / Luoma, Sari (1997). Qualitative approaches to test validation. In: Clapham, Caroline / Corson, David (eds.): Encyclopedia of Language and Education. Dordrecht: Kluwer. 275-287.

Beyer, Sabine (2005). Introspektive Verfahren im fremdsprachlichen Unterricht. Deutsch als Fremdsprache 42: 18-22.

Buck, Gary (1991). The testing of listening comprehension: An introspective study. Language Testing 8: 76-91.

$17 \mathrm{Zu}$ Forschungsdesiderata auf dem Gebiet Beurteilungsstrategien s. Arras 2007. Zu Forschungsdesiderata auf dem Gebiet test-taking strategies s. jüngst Kleppin/Reich 2009. Dort auch Versuche, test-taking strategies zielgruppengerecht zu kategorisieren. 
Cohen, Andrew D. (1987). Using verbal reports in research on language learning. In Færch, Claus / Kasper, Gabriele (eds.): Introspection in second language research. Clevedon: Multilingual Matters. 82-95.

Cohen, Andrew D. (1998). Strategies and processes in test-taking and SLA. In: Bachman, Lyle / Cohen, Andrew (eds.): Interfaces between second language acquisition and language testing research. Cambridge: Cambridge University Press. 90-111.

Cohen, Andrew D. / Upton, Thomas A. (2006). Strategies in responding to the New TOEFL reading tasks (=TOEFL Monograph Series Report No. 33). Princeton, NJ: Educational Testing Service. Online: http://www.ets.org/Media/Research/pdf/RR-06-06.pdf. (letzter Aufruf [29.07.2012])

Cohen, Andrew D. / Upton, Thomas, A. (2007). 'I want to go back to the text': Response strategies on the reading subtest of the new TOEFL. Language Testing 24: 209-250.

Dollerup, Cay / Glahn, Ester / Hansen, Carsten R. (1994). 'Sprogtest': A smart test (or how to develop a reliable and anonymous EFL reading test). Language Testing, 11/1: 65-81.

Ducasse, Ana Maria (2010). Interaction in Paired Oral Proficiency Assessment in Spanish: Rater and Candidate Input into Evidence Based Scale Development and Construct Definition. Frankfurt am Main: Peter Lang.

Ericsson, K. Anders / Simon, Herbert A. (1987). Verbal reports on thinking. In: Færch, Claus/ Kasper, Gabriele (eds.). Introspection in second language research. Clevedon: Multilingual Matters. 24-53.

Ericsson, K. Anders / Simon, Herbert A. (1993). Protocol analysis: Verbal reports as data. Cambridge: Bradford. $2^{\text {nd }}$, revised ed.

Green, Alison (1998). Verbal protocol analysis in language testing research: A handbook. Cambridge: Cambridge University Press.

Grotjahn, Rüdiger (1997). Strategiewissen und Strategiegebrauch: Das Informationsverarbeitungsparadigma als Metatheorie der L2-Strategieforschung. In: Rampillon, Ute / Zimmermann, Günter (Hrsg.): Strategien und Techniken beim Erwerb fremder Sprachen. Ismaning: Hueber. 33-76.

Grotjahn, Rüdiger. (2005). Testen und Bewerten des Hörverstehens. In: Ó Dúill, Micheál / Zahn, Rosemary / Höppner, Kristina D. C. (Hrsg.): Zusammenarbeiten: Eine Festschrift für Bernd Voss. Bochum: AKS-Verlag. 115-144.

Grotjahn, Rüdiger (2007). Lernstile/Lernertypen. In: Bausch, Karl-Richard u. a. (Hrsg.): Handbuch Fremdsprachenunterricht. Tübingen: Francke. 326-331. 5. Auflage.

Grupa Paul A. (1999). The role of digital video media in second language listening comprehension. Ph.D. thesis, University of Melbourne.

Huot, Brian (1988). The validity of holistic scoring: a comparison of the talk-aloud protocols of expert and novice holistic raters. Unpublished dissertation. Indiana: University of Pennsylvania.

Huot, Brian (1993). The influence of holistic scoring procedures on reading and rating student essays. In: Williamson, Michael M. / Huot, Brian (eds.). Validating holistic scoring for writing assessment: Theoretical and empirical foundations. Cresskill, NJ: Hampton Press. 206-236. 
Kleppin, Karin / Reich, Astrid (2009). Test-Taking Strategien. In: Berndt, Annette / Kleppin, Karin (Hrsg.): Sprachlehrforschung: Theorie und Empirie: Festschrift für Rüdiger Grotjahn. Frankfurt am Main: Peter Lang. 95-112.

Kniffka, Gabriele / Gutzat, Bärbel (2003). Training TestDaF. Material zur Prüfungsvorbereitung. Berlin: Langenscheidt.

Lodewick, Klaus (2010). TestDaF-Training 20.15. Vorbereitung auf den Test Deutsch als Fremdsprache. Göttingen: Fabouda.

Lumley, Tom (2005). Assessing second language writing: The rater's perspective. Frankfurt am Main: Peter Lang.

Lumley, Tom / Brown, Annie (2005). Research methods in language testing. In: Hinkel, Eli (ed.): Handbook of research in second language teaching and learning. Mahwah, N.J.: Lawrence Erlbaum. 833-855.

May, Lyn (2011). Interaction in a paired speaking test: The rater's perspective. Frankfurt am Main: Peter Lang.

McNamara, Tim / Hill, Kathryn / May, Lynette May (2002): Discourse and Assessment. In: Annual Review of Applied Linguistics, Volume 22 / March 2002, pp 221-242, Cambridge University Press.

Messick, Samuel (1989). Validity. In: Linn, Robert L. (ed.): Educational measurement. New York: Macmillan. 13-103. 3rd ed.

Rampillon, Ute / Zimmermann, Günther (Hrsg.) (1997). Strategien und Techniken beim Erwerb fremder Sprachen. Ismaning: Hueber.

Reason, Peter W. / Bradbury, Hilary (eds.) (2002). Handbook of action research. London: Sage Publications.

Roche, Jörg-Matthias (Hrsg.) (2005). Fit für den TestDaF: Tipps und Übungen. Ismaning: Hueber.

Rossa, Henning (2012). Mentale Prozesse beim Hörverstehen in der Fremdsprache. Eine Studie zur Validität der Messung sprachlicher Kompetenzen. Frankfurt am Main: Peter Lang.

Shohamy, Elana (2001). The power of tests: A critical perspective on the use of language tests. New York: Pearson.

Stemmer, Brigitte (1991). What's on a C-test taker's mind? Mental processes in C-test taking. Bochum: Universitätsverlag Dr. N. Brockmeyer.

TestDaF-Institut: Hinweise und Tipps zur Vorbereitung. Online: http://www.testdaf.de/teil nehmer/tn-vorbereitung_tipp.php (letzter Aufruf [29.07.2012]).

Wu, Yi'an (1998). What do tests of listening comprehension test? A retrospection study of EFL test-takers performing a multiple-choice task. Language Testing 15: 21-44. 


\title{
Lautes Denken im Licht der Embodied Cognition: neue Grenzen, neue Möglichkeiten in der Schreibprozessforschung?
}

\section{Anna Katharina Schnell}

\begin{abstract}
In the last three decades, think-aloud has become a popular method to collect data in several research fields. Until recently the basic issues of mental information processing during the process of thinking aloud seemed to be fairly clear. Due to the latest paradigm shift in cognitive psychology, however, some fundamental methodological questions have to be revisited. Specifically the emerging research disciplines 'Embodied Cognition' and 'Distributed Cognition' propose a substantially new perspective on human information processing. Utilising think-aloud data from a study on second language writing, I will attempt to evaluate whether these new research perspectives can make a contribution to the discussion of think-aloud methodology.
\end{abstract}

\section{Einleitung}

Obwohl es zum Verfahren des Lauten Denkens ${ }^{1}$ in den vergangenen rund 30 Jahren einige kontroverse methodologische Diskussionen gab, hat sich das Laute Denken mittlerweile u. a. in der (fremdsprachlichen) Schreibprozessforschung, in der Übersetzungsprozessforschung, in einigen Bereichen der kognitiven Psychologie sowie in der Sprachlehr- und -lernforschung als anerkannte Erhebungsmethode etablieren können. Der Einsatz des Lauten Denkens eröffnet je nach Erhebungssituation, untersuchten Informantengruppen, Forschungsfragen und Bezugsmodellen unterschiedlichste Einblicksmöglichkeiten in menschliche Denkund Verhaltensweisen. ForscherInnen, die mit der Methode des Lauten Denkens arbeiten, sind deshalb u. a. mit einigen grundsätzlichen Fragen zu menschlichen Repräsentations- und Verarbeitungsmechanismen konfrontiert. In den letzten drei Jahrzehnten wurde bei dieser methodologischen Diskussion vor allem auf verhältnismäßig einfache kognitionspsychologische Informationsverarbeitungsmodelle rekurriert. Ausgelöst durch einen Paradigmenwechsel in der Kognitiven Psychologie müssen nun allerdings grundlegende Fragen zu den inneren Verarbeitungsmechanismen beim Lauten Denken neu diskutiert werden. Insbesondere die Einbeziehung emotionaler, situationaler und sozialer Prozesse in die Diskussion zur menschlichen Informationsverarbeitung scheint in Anbetracht

\footnotetext{
${ }^{1}$ Unter Lautem Denken verstehe ich ,eine spezielle Form der Introspektion, die sich [...] dadurch auszeichnet, dass die Versuchspersonen alles, was ihnen bei der Ausführung einer konkreten Aufgabe durch den Kopf geht [...], periaktional [...], spontan und unselektiert äußern [...], wobei der Versuchsleiter möglichst wenig [bzw. gar nicht] eingreifen sollte [...]) “(Göpferich 2008: 22).
} 
neuer kognitionspsychologischer und neuropsychologischer Forschungserkenntnisse unausweichlich. Eine erste Annäherung an dieses Thema soll im Rahmen dieses Beitrags unternommen werden.

Im Folgenden gehe ich zunächst kurz auf die methodologischen Grundlagen ein, die Mitte der 1980er Jahre von Ericsson/Simon zum Verfahren des Lauten Denkens erarbeitet wurden, da sie bis heute für viele ForscherInnen, die mit dem Verfahren des Lauten Denkens arbeiten, als Grundlage dienen. Anschließend stelle ich kurz neuere kognitionspsychologische Ansätze vor - insbesondere die Embodied und Distributed Cognition - und prüfe ihre Bedeutung für die Bewertung von Lautdenkdaten. Diskussionsgrundlage sind Ausschnitte aus Lautdenkdaten, die im Rahmen einer Untersuchung zum fremdsprachlichen Schreiben elizitiert wurden.

\section{Methodologische Grundlagen des Lauten Denkens}

Die methodologische Diskussion zum Verfahren des Lauten Denkens bewegt sich seit nun rund 30 Jahren um mehrere wichtige Fragen (siehe dazu auch Arras in diesem Band). Die erste - und m. E. wichtigste - Frage ist, was prinzipiell durch das Laute Denken erhoben werden kann und - daraus resultierend - wie relevant und umfassend diese Einblicke in Anbetracht unterschiedlicher Forschungsfragen sind. Ein zweites bedeutsames Thema ist zudem, ob und wenn ja, wie die Verbalisierungsaufgabe die normale Bearbeitung der Primäraufgabe beeinflusst (Reaktivität). Darüber hinaus stellte sich in vorangegangenen Untersuchungen heraus, dass sich Menschen hinsichtlich ihrer Verbalisierungsfähigkeit unterscheiden, wobei die Einblicksmöglichkeiten sogar innerhalb derselben Aufgabenbearbeitung intraindividuell schwankend sind. Auch dieses Phänomen muss bei einer methodologischen Diskussion zum Lauten Denken bedacht werden. Wenn Lautes Denken in der Fremdsprachenforschung eingesetzt wird, kommt darüber hinaus noch die Frage hinzu, ob in der L1 oder in der L2 laut gedacht werden sollte (vgl. dazu z. B. Ericsson/Simon 1993: 249f., Heine 2010: 90f., Heine/Schramm 2007) und - im Fall, dass dieses den InformantInnen freigestellt wird - wie die im Vorgespräch verwendete Sprache (L1 oder L2) die Lautdenksprache (L1 oder L2) der InformantInnen bei der Aufgabenbearbeitung beeinflusst (vgl. Heine 2005, 2010). Die Diskussion zu den Fragen nach den Interferenzen $^{2}$ zwischen der Verbalisierungsaufgabe und der Primäraufgabe, zur

\footnotetext{
${ }^{2}$ Erwähnt sei, dass in jüngster Zeit eine statistische Metaanalyse zu Studien, die im Bereich der Zweit- und Fremdsprachenforschung mit dem Verfahren des Lauten Denkens gearbeitet haben, vorgelegt wurde (Bowles 2010). Die Forscherin konnte in ihrer Untersuchung u. a. nachweisen, dass die Leistungen von InformantInnen, die gebeten wurden, ihre Gedanken bei einer sprachlichen Aufgabe zu verbalisieren, nicht signifikant besser oder schlechter abschnitten als die Kontrollgruppen, welche die Aufgabe ohne Lautes Denken bearbeiteten.
} 
inter- und intraindividuell unterschiedlichen Verbalisierungsfähigkeit sowie zum Einfluss der Rahmenbedingungen auf eine Lautdenkuntersuchung kann aus Platzgründen hier nicht im Einzelnen nachgezeichnet werden (ausführliche methodologische Diskussionen zu den o. g. Themen finden sich z. B. bei Bowles 2010, Ericsson/Simon 1993, Heine 2005, 2010, Heine/Schramm 2007, Krings 1986, 1994). Im Folgenden werde ich mich zunächst vor allem mit der Frage beschäftigen, welche Einblicke prinzipiell durch das Laute Denken ermöglicht werden. Dazu stelle ich kurz die kognitionspsychologischen Annahmen vor, auf welche in den letzten Jahrzehnten im Rahmen der Diskussion zum Lauten Denken am häufigsten rekurriert wurde, um diese anschließend im Rahmen neuerer kognitionspsychologischer Entwürfe zur menschlichen Informationsverarbeitung zu diskutieren.

\section{Ein Klassifikationsschema zur Validität von Lautdenkdaten}

Die Frage, was grundsätzlich durch das Verfahren des Lauten Denkens erhoben werden kann, ist nicht banal. Bis heute rekurrieren viele ForscherInnen, die sich mit den methodologischen Grundlagen des Verfahrens des Lauten Denkens auseinandersetzen, auf die theoretische Basis, welche die amerikanischen Psychologen K. A. Ericsson und H. A. Simon 1984 (in einer überarbeiteten Version 1993) zum Lauten Denken, aber auch zu verbalen Daten insgesamt vorgelegt haben (vgl. z. B. Bowles 2010, Beare/Bourdages 2007, Chi 1997, Heine 2005, 2010, Heine/Schramm 2007, Krings 1986, 1994). Ericsson/Simon (1993) blicken bei Veröffentlichung ihrer Monografie auf eine rund 20-jährige Forschungspraxis mit verbalen Daten zurück, beschäftigen sich mit Metaanalysen zur Validität verbaler Daten und setzen sich zudem ausführlich mit den Positionen der Gegner verbaler Daten auseinander. Darauf aufbauend entwickeln sie unter Rückgriff auf ein (bewusst einfach gewähltes) kognitionspsychologisches Informationsverarbeitungsmodell ein Klassifikationsschema zur Güteeinschätzung verschiedener Arten verbaler Daten im Allgemeinen und zu Lautdenkdaten im Besonderen: Nach Einschätzung der Forscher können kognitive Prozesse, die im Kurzzeitgedächtnis in nicht automatisierter, sprachlich kodierter Form ablaufen, ohne bedeutende Verzögerung und Verzerrung verbalisiert werden. Ericsson und Simon bezeichnen diese Äußerungen als level1 -Verbalisierungen und erwarten ein hohes $\mathrm{Ma}$ an Validität für diese Verbalisierungsart (Ericsson/Simon 1993: 79). ${ }^{3}$ Wenn Informationen im Kurz-

Siehe hierzu auch den Forschungsüberblick speziell in Bezug auf Interferenzen beim Übersetzen in Göpferich (2008: 27f.)

${ }^{3}$ Gängige Sprachproduktionsmodelle (z. B. Levelt et al. 1999) widersprechen allerdings diesem Befund, da empirische Evidenz besteht, dass Enkodieren und Dekodieren unabhängige Prozesse darstellen. Der eigene Output wird zum neuen Input, wenn er laut ausgesprochen bzw. subvokalisiert wird. Es wird jedoch angenommen, dass SchreiberInnen diesen Rück- 
zeitgedächtnis nicht in sprachlicher Form, sondern z. B. bildhaft, emotional, olfaktorisch etc. gespeichert sind, aber kurz nach dem Auftreten versprachlicht (rekodiert) werden, sprechen die beiden Forscher von level-2-Verbalisierungen. Durch die zeitliche Nähe zu der entsprechenden Aktivität können diese Auskünfte der InformantInnen ebenfalls als recht valide betrachtet werden. Allerdings muss durch den Rekodierungsaufwand damit gerechnet werden, dass diese Verbalisierungen weniger vollständig auftreten als die level-1-Verbalisierungen (vgl. dazu auch Krings 1994). Die Reihenfolge der Prozesse wird jedoch nach Ansicht von Ericsson/Simon (1993) bei der Rekodierung nicht verändert. Es soll vor allem die Zeit, die für die Rekodierung benötigt wird, sein, die dazu führt, dass InformantInnen, die Aufgaben unter Lautdenk-Bedingungen bearbeiten, wesentlich länger brauchen als Kontrollgruppen, die dieselben Aufgaben ohne Verbalisierungsaufgabe bearbeiten (vgl. dazu auch Bowles 2010, eine leicht kritische Einschätzung bieten auch Krings 1994: 213 und Cohen 1998). Wenn von InformantInnen im Rahmen der Verbalisierung zusätzlich Abstraktionen, Filterungen, Selektionen etc. verlangt werden, sprechen die Forscher von level3-Verbalisierungen. Bei diesem Verbalisierungstyp gibt es größere Validitätsprobleme. Wenn dieser Verbalisierungstyp im Rahmen von periaktionalem Verbalisieren auftritt, können erhebliche Einflüsse auf die Bearbeitung der Primäraufgabe nicht ausgeschlossen werden (vgl. dazu auch Heine/Schramm 2007). Nachdrücklich betonen die Forscher, dass keiner der Verbalisierungstypen als direkte Widerspiegelung der mentalen Verarbeitung gesehen werden sollte, sondern es liege an dem/der ForscherIn, aus den teilweise sehr fragmentarischen Äußerungen der InformantInnen den Zusammenhang zu mentalen Aktivitäten zu rekonstruieren. Da sich Lautdenkdaten - wenn sie richtig erhoben werden - vor allem aus level-1- und level-2-Verbalisierungen zusammensetzen, prognostizieren die Forscher für diese Daten ein hohes Maß an Validität.

Will man nun mithilfe dieser grundsätzlichen methodologischen Einordnungen von Ericsson und Simon (1993) eine Einschätzung zu Lautdenkdaten vornehmen, muss man zunächst einen Blick darauf werfen, welche Anteile der menschlichen Kognition bei der Bearbeitung unterschiedlicher Aufgaben grundsätzlich potentiell nicht automatisiert ablaufen könnten. Bedingt durch einen Paradigmenwechsel in der Kognitiven Psychologie lohnt es sich, diese Frage grundsätzlich neu zu beleuchten.

kopplungseffekt - insbesondere beim Formulieren - auch ohne Aufforderung zum Lauten Denken - sehr häufig nutzen (vgl. dazu auch Krings 1994). Sasaki (2008), Smagorinsky (2001) und Swain (2006) haben mit Bezug auf soziokulturelle sprachtheoretische Überlegungen ebenfalls darauf hingewiesen, dass Sprechen immer eine bedeutungskonstruierende Funktion hat - eine Tatsache, die ebenfalls stärker in die methodologische Diskussion zum Lauten Denken einbezogen werden muss (siehe dazu auch Knorr in diesem Band, Feick in diesem Band). 


\section{Paradigmenwechsel in der Kognitiven Psychologie}

In der Zeit, in der Ericsson und Simon ihre Monografie vorlegten, herrschte in der Kognitionsforschung noch ein stark durch die Computerwissenschaften beeinflusstes Subjektmodell vor. Menschliches Denken wurde in diesem Zuge als serielle, amodale und abstrakte Symbolmanipulation modelliert. Erste Konkurrenz zu dieser Konzeption kam bereits Mitte der 1980er Jahre durch Modelle auf, die von einer parallelen Verarbeitung verschiedener Informationen ausgingen. Diese sogenannten konnektionistischen Modelle waren jedoch häufig derartig vielschichtig und unübersichtlich, dass sie schnell an forschungspraktische Grenzen stießen. Aus diesem Grund sind sie auch bis heute wenig verbreitet (vgl. Betsch et al. 2011: 20). Seit kurzem zeichnet sich nun jedoch ein tiefgreifender Paradigmenwechsel hinsichtlich der subjekttheoretischen Annahmen der Kognitionspsychologie ab. Unter Namen wie z. B. Grounded Cognition, Embodied Cognition (auch Embodiment) und Distributed Cognition werden Ansätze dominant, die stärker emotionale, soziale und situationale Aspekte der menschlichen Informationsverarbeitung in den Blick nehmen (vgl. z. B. Barsalou 2008, Gendron et al. 2012, Shapiro 2011; gute Einführungen in das Thema bieten z. B. Rickheit et al. 2010, Schwarz 2008). Im Rahmen dieser second generation cognitive science (Sinha 2010) wird auch zunehmend grundsätzliche Kritik an den traditionellen Informationsverarbeitungsmodellen der Kognitiven Psychologie geübt:

Standard cognitive scientists see the brain as the complete organ of cognition because they take cognition to consist in the algorithmic processes that mediate between a symbolically encoded stimulus and a symbolically encoded response. But the possibility that an organism's interactions with the environment can suffice to extract invariants from stimuli suggests an alternative conception of the locus of cognition. The assumption that the brain functions as an inference engine begins to look misguided. Still correct is the idea that cognition involves "intelligent" responses to stimuli, but because the detection of stimuli requires active exploration of the environment, the organs and processes of cognition extend beyond the brain in which information about the environment is collected. The brain, in this view, is better conceived as a controller and organizer of activities that result in the extraction of information than as a computer for processing this information. (Shapiro 2011: 49)

Auch im Rahmen dieser neuen Ansätze wird von parallelen und vielschichtigen Verarbeitungs- und Repräsentationsformen ausgegangen:

Im Gegensatz zu den klassischen Ansätzen der Kognitionswissenschaft beruht die Annahme modaler Kognitionstheorien darauf, dass Sprache in multiplen, verteilten Repräsentationen verankert ist und auf kognitiven Fähigkeiten, wie Wahrnehmung, Handlung und Introspektion (innere mentale- und Gefühlszustände) beruht [...]. (Hervorhebungen i. O.; Rickheit et al. 2010: 105)

Die Hauptthese ist dabei, dass dem gesamten kognitiven Erleben sogenannte ,multimodale mentale Simulationen’ zugrunde liegen, die im Laufe der Ontoge- 
nese erworben wurden und in aktuellen Situationen reaktiviert werden. Dabei können je nach Kontext in unterschiedlichem Maß sensorische Perzeptionen, körperliche, introspektive und affektive Zustände sowie situierte Handlungen, Weltwissen und mentale Zustände anderer Personen eine Rolle spielen. Rickheit et al. (2010) erläutern am Beispiel der Verarbeitung des Begriffs ,Katze' wie man sich multimodale mentale Simulationen vorstellen kann:
Alle bisherigen Erfahrungen mit einem bestimmten Begriff, beispielsweise dem wahr- genommenen Wort ,Katze', bewirken, dass im Gehirn multimodale Repräsentationen dieses Begriffes aufgebaut werden: etwa das Streicheln einer Katze, ihr Abbild, das Spiel mit ihr, das Fühlen der Krallen, ihr Geruch, das Hören des Schnurrens usw. Wenn nun das betreffende Konzept während der Sprachverarbeitung umgesetzt werden muss, werden diese multimodalen Erfahrungen automatisch reaktiviert und ermöglichen dem Gehirn dadurch eine Simulation der relevanten Informationen. Dabei ist zu beachten, dass Simulationen aus vielen dynamischen Komponenten bestehen und keine holisti- sche Abbildung eines Objekts oder einer Situation liefern [...]. Sie werden eher als multimodale schematische Spuren gesehen [...] und müssen uns nicht zwingend immer bewusst werden [...]. (Rickheit et al. 2010: 107)

In der Kognitiven Linguistik wurden im Rahmen dieser veränderten Annahmen zur menschlichen Informationsverarbeitung in den letzten Jahren etliche interessante Priming-Experimente durchgeführt. Die Ergebnisse dieser Experimente untermauern die These der starken Beteiligung von u. a. emotionalen, situationalen und sozialen Komponenten an der menschlichen Sprachverarbeitung (einen guten Forschungsüberblick geben ebenfalls Rickheit et al. ebd.: 108f.; interessante theoretische Überlegungen zu Sprache und Emotion finden sich auch in Schwarz-Friesel 2007). Erwähnt sei in diesem Zusammenhang zudem, dass auch viele NeurowissenschaftlerInnen seit einigen Jahren nachdrücklich darauf hinweisen, wie sehr kognitive Leistungen und rationale Handlungen von emotionalen Prozessen beeinflusst werden (vgl. z. B. Damasio 2006, Damasio/Kober 2007, 2009, Panksepp 2004, Roth 2004, 2009).

Was bedeutet nun aber eine derart andere Konzeption der menschlichen Informationsverarbeitung sowohl für die grundsätzliche methodologische Diskussion zum Lauten Denken als auch für die Auswertung von Lautdenkdaten? Diese Frage kann natürlich erst dann abschließend geklärt werden, wenn vollständige, empirisch geprüfte Modelle von der Kognitionspsychologie vorgelegt und/oder speziell auf diesen Aspekt ausgerichtete methodenbezogene Studien durchgeführt werden. Zudem betreffen diese Umbrüche nicht nur das Laute Denken an sich, sondern auch immer das Gegenstandsmodell und/oder die Beschreibungsebene, die einer Untersuchung zugrunde gelegt werden (siehe auch Abschnitt 3.4). Dennoch möchte ich den Versuch wagen, diese neuen subjekttheoretischen Vorstellungen im Hinblick auf die Einblicksmöglichkeiten des Lauten Denkens bei der Herstellung eines fremdsprachlichen Textes zu diskutieren: Hält man weiter an der Annahme von Ericsson/Simon (1993) fest, dass über Lautes Denken (nicht sprachliche) nicht automatisierte Prozesse über Rekodierung und 
nicht automatisierte sprachliche Kurzzeitgedächtnisinhalte direkt verbalisiert werden können (siehe Abschnitt 2), muss im Rahmen der o. g. neuen subjekttheoretischen Annahmen davon ausgegangen werden, dass zusätzlich zu den über die Verbalisierungen freigelegten, nicht automatisierten Kurzzeitgedächtnisinhalten noch etliche weitere innere Verarbeitungsmechanismen vermutet werden können. Grundsätzlich lässt die Modellierung von Ericsson und Simon zwar auch zu, dass nichtsprachliche Prozesse, die ins Kurzzeitgedächtnis gelangen, über Rekodierung versprachlicht werden können, es muss allerdings vermutet werden, dass nur ein sehr geringer Teil dieser inneren Vorgänge bewusst abläuft. Man bedenke, dass (erwachsene) Menschen eine jahrzehntelange Gefühls-, Wahrnehmungs-, Situations- und Sprachsozialisation durchlaufen haben. Hinzu kommt, dass sich im Rahmen der neuen Modelle auch die Annahmen hinsichtlich der Natur der menschlichen Gedanken ändern. Wo früher von seriellen Gedankenketten ausgegangen wurde, geht man heute davon aus, dass das Denken sprunghaft, multimodal und synkretistisch (vgl. Boeckx 2010, Ortner 2003b) verläuft und nicht unwesentlich durch die Aufgabenstellung beeinflusst wird (vgl. Molitor-Lübbert 2003, Ortner 2000, 2003a, 2003b, 2007). Jedenfalls ist relativ sicher, dass „das Denken [...] im Normalfall schneller [verläuft] als das Sprechen“" (Aguado 2004: 28). Es kann also vermutet werden, dass Verarbeitungsmechanismen, wie z. B. emotionale, bildliche, sensomotorische Simulationen, die ins Bewusstsein von InformantInnen gelangen, wahrscheinlich eher wie ein schnelles Aufblitzen wahrgenommen werden.

Die erste Frage, die in diesem Zusammenhang im Folgenden beantwortet werden soll, ist, ob sich Spuren von multimodalen Simulationen in Lautdenkdaten finden lassen und ob es Hinweise darauf gibt, dass diese Auswirkungen auf die Aufgabenbearbeitung haben (Abschnitte 3.1; 3.2; 3.3). Diese Diskussion findet auf Grundlage von Beispielen aus Lautdenkdaten statt, die ich im Rahmen meines Promotionsprojekts zur Entwicklung des fremdsprachlichen Schreibverhaltens von Studierenden erhoben habe (vgl. Schnell in Vorbereitung). ${ }^{4} \mathrm{Im}$ Anschluss daran wird kurz dargestellt, welche Phänomene durch das Laute Denken in Verbindung mit der Herstellung eines fremdsprachlichen Textes freigelegt werden können und welche weiteren Grenzen dabei auftreten.

\footnotetext{
${ }^{4}$ Es handelt sich um Lautdenkdaten von Studierenden (Frankoromanistik, Bachelor), die zu der Fragestellung La vie d'un étudiant est-elle préferable à celle d'un lycéen? dt. „Ist das Studentenleben dem Schülerdasein vorzuziehen?" (Übersetzung AKS) einen Text mit mindestens 150 Wörtern schreiben sollten. Die Studierenden waren während der gesamten Schreibzeit allein in einem Raum und durften ein- und zweisprachige Print- und OnlineWörterbücher sowie eine Grammatik benutzen. Zusätzlich zu den Lautdenkdaten wurden Bildschirmvideos des gesamten Schreibprozesses aufgezeichnet. Die in diesem Rahmen erfassten Schreibhandlungen wurden in den Lautdenkprotokollen in eckigen Klammern eingetragen (siehe Beispiele). Vor und nach der Erhebung der Lautdenkdaten wurden die Studierenden ausführlich zu ihren Lese-, Schreib- und Sprachlernerfahrungen befragt.
} 


\subsection{Sensorische Perzeptionen in Lautdenkprotokollen}

Sensorische mentale Simulationen lassen sich auf den ersten Blick nur in sehr seltenen Fällen in den Verbalisierungen erkennen. Folgend sind einige der wenigen Beispiele aufgeführt, die ich in meinem Korpus finden konnte:

1. [...] la vie (singt) lala* ((lachend)) da gibts es doch auch so $\mathrm{n}$ Lied* $((1,1 \mathrm{~s}))$ oder nee das fängt mit l'amour an na egal la vie [...] (InformantIn A ft2).

2. [...] ensourceler ach ne da hab ich doch gestern noch $\mathrm{n}$ Lied gehört [...] (InformantIn $\mathrm{B} \mathrm{ft} 1)$.

3. [...] wir hatte sogar mal so $\mathrm{n}$ ekligen Lehrer $((1,1 \mathrm{~s}))$ toll jetz is mir schlecht $[\ldots]$ (InformantIn $\mathrm{C} \mathrm{ft1)}$.

Im ersten Beispiel kam es zu einer inneren Simulation eines Liedes; im zweiten Beispiel vermutlich ebenso. Im dritten Beispiel hat der Gedanke an einen Lehrer sogar eine körperlich-emotionale Reaktion bei einem Informanten ausgelöst. Auf den ersten Blick erscheinen diese Verbalisierungen allerdings wie nachgelagerte Assoziationsmomente, über die man kaum etwas über die inneren Generierungsprinzipien erfahren kann. Zudem sind sie - wie bereits erwähnt - sehr selten bzw. bei vielen InformantInnen gar nicht in den Lautdenkprotokollen zu finden. Man könnte damit vermuten, dass Lautdenkdaten gar keine Chance bieten, derartige innere Verarbeitungsmechanismen zu untersuchen. M. E. gibt es neben der direkten Beobachtung aber noch einen anderen Weg, über welchen man sich derartigen Phänomenen in Lautdenkdaten annähern kann: Es ist zu bedenken, dass Lautdenkdaten aus Sprache bestehen. Sprache an sich ist ihrerseits ebenfalls ein kognitives Produkt, in dem bereits eine Reihe emotionaler, situationaler und sozialer Prinzipien eingeschrieben sind. Unter Rückgriff auf sprachtheoretische bzw. diskursanalytische Überlegungen kann damit u. U. doch ein indirekter Zugriff auf $u$. a. auch unbewusste Verarbeitungsmechanismen ermöglicht werden. Beispielsweise ist in Beispiel 3 auffällig, dass die Informantin von „wir“ spricht. Es kann vermutet werden, dass sie sich gerade in der Schule sieht, umgeben von ihren KlassenkameradInnen, konfrontiert mit einem „ekligen Lehrer“. Auch im folgenden vierten Beispiel - einer inhaltlichen Planungssequenz - spricht die Informantin interessanterweise davon, ihre „Schwester [zu] sehe[n]“. Dieses wirft die Hypothese auf, dass die Informantin wirklich für einen kurzen Moment ihre stetig lernende Schwester visualisiert:

4. [...] joa was noch? $((2,0 \mathrm{~s}))$ keine Ahnung $((1,1 \mathrm{~s}))$ ich war immer total gestresst auf jeden Fall $((1,7 \mathrm{~s}))$ mhh ((leichtes Kauen beim Reden $))$ wenn ich meine Schwester sehe was die alles lernen muss worauf sie überhaupt keine Lust hat $((2,0 \mathrm{~s})) \mathrm{mhh}((2,4 \mathrm{~s}))$ joa $((2,4 \mathrm{~s}))^{*}$ ja wirklich dieser Stress einfach ne' [le stress] [...] (InformantIn B ft1).

Tenbrink (2010) argumentiert, dass gerade Lautes Denken eine besonders geeignete Sprachproduktionssituation darstellt, um über sprachliche Eigenschaften innere Repräsentations- und Verarbeitungsmechanismen zu untersuchen: 
$\mathrm{Da}$ insbesondere Think-Aloud-Daten (und in eingeschränkter Form auch retrospektive Berichte) ausdrücklich nicht zum Zwecke der Kommunikation (zur Erreichung bestimmter Diskursziele) ${ }^{5}$, sondern zur Repräsentation interner Vorgänge produziert werden, liegt nahe, dass prominente Aspekte in den Sprachdaten prominente Aspekte in den internen Repräsentationen widerspiegeln. (Hervorhebung i. O.; Tenbrink 2010: 6)

Insbesondere die inhaltlichen Planungssequenzen, die man in Lautdenkprotokollen zum (fremdsprachlichen) Schreiben finden kann (siehe auch Beispiel 15), bieten eine große Anzahl sprachlich interessanter Phänomene und würden sich damit m. E. gut für unterschiedlichste sprachbezogene Analysen eignen. Es ist deshalb erstaunlich, dass bislang nur selten versucht wurde, in Lautdenkprotokollen sprachliche Eigenschaften zu identifizieren, die sich als Indikatoren für innere Verarbeitungsmechanismen eignen:

Sprache wird bei der Elizitierung und Analyse von Think-Aloud-Daten und retrospektiven Berichten für gewöhnlich ausschließlich als Vehikel zur Vermittlung von Inhalten betrachtet. Die sprachliche Struktur bzw. die Form, in der diese Inhalte transportiert werden, und die systematischen Eigenschaften der frei produzierten Sprache werden dabei typischerweise nicht berücksichtigt. (Tenbrink 2010: 6)

Über die stärkere Einbeziehung linguistischer und/oder diskurstheoretischer Analysemethoden würde sich also ein völlig neuer Weg eröffnen, sowohl aufgabenbezogene Erkenntnisse zu erzielen als auch die Diskussion zu grundsätzlichen inneren Verarbeitungsmechanismen zu bereichern. Es wäre wünschenswert, wenn in diesem Rahmen nicht nur kognitionspsychologische, sondern verstärkt auch soziokulturelle sprachtheoretische Überlegungen berücksichtigt werden (siehe dazu auch Sasaki 2008, Smagorinsky 2001, Swain 2006, Feick in diesem Band, Knorr in diesem Band). Das einzige mir bekannte Projekt, das derzeit einen linguistisch-diskursanalytischen Ansatz in Kombination mit Lautdenkdaten verfolgt, ist im Bremer Sonderforschungsbereich Spatial Cognition beheimatet (siehe ebenfalls Tenbrink 2010; erstmals vorgeschlagen wurde dieses Vorgehen m. W. übrigens von Caron/Caron-Pargue 1986). Solange derartige Analysen und die dazugehörigen theoretischen Überlegungen für das (fremdsprachliche) Schreiben noch ausstehen, kann an dieser Stelle natürlich die Frage nach den Auswirkungen unterschiedlicher sensorisch-perzeptueller Verarbeitungsmechanismen auf den Ablauf der Aufgabenbearbeitung an sich nicht geklärt werden. Festgehalten werden kann jedoch, dass in den von mir erhobenen Lautdenkprotokollen allein durch eine verhaltensbasierte Analyse kaum Erkenntnisse zu sensorisch-perzeptuellen Verarbeitungsmechanismen gewonnen werden können (siehe auch nächster Abschnitt).

\footnotetext{
${ }^{5}$ Sasaki (2008) und Smagorinsky (2001) plädieren allerdings für eine andere Sichtweise. Mit Bezug auf soziokulturelle sprachtheoretische Überlegungen, gehen die ForscherInnen davon aus, dass jegliche Form der Versprachlichung eine selbst- oder fremdadressierte dialogische Handlung darstellt (vgl. auch Feick in diesem Band, Knorr in diesem Band).
} 


\subsection{Emotionale Prozesse in Lautdenkprotokollen}

Auch wenn emotionale Prozesse m. E. teilweise zu den sensorisch-perzeptuellen mentalen Simulationen gerechnet werden können (siehe unten), ${ }^{6}$ werden sie an dieser Stelle gesondert diskutiert, da sie m. E. eine zentrale Rolle für das Handlungsgeschehen spielen, wie an folgenden Beispielen verdeutlicht werden soll:

5. [...] aller au bout de ses forces $((1,1 \mathrm{~s}))$ klingt doch gar nicht so schlecht $((2,1))$ joa das nehm ich mal [...] (InformantIn D ft1).

6. [...] Verantwortlichkeit Eigenverantwortlichkeit responsabilité personnelle ja ja das gefällt mir [...] (InformantIn B ft1).

7. [...] la vie étudiante geht das? mh:, kenn ich das' la vie étudiante $((1,1 \mathrm{~s}))$ la vie étudiante hab ich irgendwie n ungutes Gefühl $((1,2 \mathrm{~s}))$ étudiante $((1,4 \mathrm{~s}))$ gucken wa das doch lieber nach [...] (InformantIn E ft1).

8. [...] premier pas öh für die Zukunft pour in Richtung Zukunft ähm en direction keine Ahnung d'avenir [on direction $\mathrm{d}$ ] nee das sacht $\mathrm{k} /$ das hört sich komisch an $\mathrm{mh}((1,4 \mathrm{~s}))$ en direction $((1,2 \mathrm{~s})) \mathrm{mh}, \mathrm{mh}, \mathrm{mh},[\ldots]$ (InformantIn B ft3).

In den Beispielen 5 und 6 schlagen die beiden InformantInnen einen muttersprachlichen Ausdruck in einem Wörterbuch nach und bewerten jeweils die gefundenen L2-Äquivalente. Die Bewertungen „klingt gut“, „gefällt mir“ u. Ä. lassen sich in den von mir erhobenen Lautdenkprotokollen zum fremdsprachlichen Schreiben vergleichsweise häufig finden. Wie allerdings genau diese Gefühls- bzw. Klangprobe innerlich vollzogen wird, ob sie sich interindividuell oder sogar von Fall zu Fall unterscheidet, aus welchen Informationen sie gespeist wird, wie häufig sie z. B. auch während des Formulierens und Lesens vorkommt etc., kann über das Laute Denken nicht ermittelt werden. Auch Aguado (2004) stellt die Frage,

inwiefern die [beim Lauten Denken, AKS] zur Verfügung stehenden sprachlichen Mittel ausreichen, die differenzierten, veränderlichen und flüchtigen mentalen bzw. emotionalen Vorgänge und Zustände zutreffend, adäquat oder intersubjektiv nachvollziehbar zu beschreiben. (Aguado 2004: 28)

An diesem Punkt stoßen Lautdenkdaten bei der Rekonstruktion der an der Aufgabe beteiligten emotionalen Prozesse an eine empfindliche Grenze. Es besteht jedoch m. E. kein Zweifel daran, dass diese emotionalen Bewertungsprozesse maßgeblich das Handlungsgeschehen steuern. Anhand der Klangprobe entscheidet sich beispielsweise sehr häufig, ob Ausdrücke, phraseologische Verbindungen, Konstruktionen etc. als akzeptabel eingestuft und verwendet werden oder ob weitere Suchen eingeleitet werden, wie an den Beispielen 7 und 8 deutlich

${ }^{6}$ Es ist selbstverständlich grob vereinfacht, von „emotionalen Prozessen“ zu sprechen. Eigentlich müsste wenigstens zwischen Empfindungen, Gefühlen, Affekten, Zuständen und ggf. Trieben unterschieden werden, die vermutlich ihrerseits ebenfalls ein komplexes System bilden. Für eine erste Annäherung sollte meine Verallgemeinerung allerdings dienlich sein. Ein sehr interessantes ganzheitliches Modell zu inneren Verarbeitungsmechanismen wurde übrigens von dem Traumaforscher Peter Levine entwickelt (siehe Levine/Petersen 2011). 
sichtbar wird. Emotionale Prozesse scheinen allerdings auch bei der Gesamtgestaltung eines Textes eine wichtige Rolle zu spielen:

9. [...] moment irgendwas stimmt hier nicht c'est moi qui décide ce que je veux faire' $((1,2 \mathrm{~s}))$ j'ai beaucoup de l'autonomie dans ma vie'/ das passt doch irgendwie nich $((2,2 \mathrm{~s}))$ ähm $((1,5 \mathrm{~s}))$ das ist irgendwie doppelt $((2,1 \mathrm{~s}))$ oder ich brauch einen Übergang [...] (InformantIn D ft2).

10. [...] oh Gott das is jetz irgendwie komisch $((1,2 \mathrm{~s}))$ ich weiß gar nicht mehr was die Frage war $((4,2 \mathrm{~s}))$ ach préférable $((1,6 \mathrm{~s}))$ dann stimmt das nich moment $[\ldots]$ (InformantIn B ft1).

11. [..] A:H: wenn ich das jetzt noch reinbringe ist dann oben wieder alles Chaos $[\ldots]$ (InformantIn F ft2).

Besonders in Beispiel 9 wird deutlich, dass zunächst ein Gefühl da ist, dass „irgendwas nicht stimmt“, und erst im nächsten Schritt analysiert der Informant das Problem genauer. Aber auch die Beispiele 10 und 11 legen die Vermutung nahe, dass Emotionen nicht nur (bewussten) Gedanken nachgelagerte Prozesse sind, sondern SchreiberInnen wichtige Hinweise zu Problemen liefern, die sie bewusst (analytisch) noch gar nicht durchdrungen haben. Einer der wenigen Schreibforscher, der sich im Rahmen einer Untersuchung zu Schreibstrategien von SchriftstellerInnen ebenfalls mit der Rolle von Gefühlen und Empfindungen beim Schreiben beschäftigt hat, ist Ortner (2000):

Jeder Schreiber, fast jeder, weiß irgendwann, daß er mit seinem Text zu Rande gekommen ist (oder eben noch nicht). Warum weiß er das? Wie denn anders als gefühlsmäßig sollte er wissen, ob er sein ihm mögliches Äquilibre erreicht hat? Er spürt es; er weiß es intuitiv, aber er kann es rational kaum begründen, und er kann es - es analysierend kaum in Worte fassen.

Die gefühlsmäßige Bewertung verfestigt sich langfristig in Motiven, Einstellungen und Dispositionen. Diese werden in ihrer Gesamtheit zu einer Art innerem Kompaß, der bei der Aktualgenese als Leitinstanz immer dabei ist. (Ortner 2000: 161)

Es ist m. E. äußerst plausibel, dass unterschiedlichste sprach- und schreibbezogene Erfahrungen, die ein(e) SchreiberIn im Laufe der Schreib- und Lesesozialisation gesammelt hat, in großen Teilen nicht bewusst vorhanden sind, sondern in Form von Gefühlen und Empfindungen gespeichert wurden. Diese werden dann beim Schreiben als ,irgendetwas stimmt noch nicht“, „,da passt was nicht“, „das hört sich nicht gut an“, aber auch „genau das ist es, was ich sagen wollte" wahrgenommen. Aufgrund welcher Erfahrungen und Kenntnisse ein/e SchreiberIn genau zu diesen Bewertungen kommt, kann ad hoc wahrscheinlich in den wenigsten Fällen ermittelt werden. Levine/Petersen (2010) gehen davon aus, dass unterschiedliche, in der Ontogenese erworbene Erfahrungen in subtilen, meist nur unterschwellig wahrgenommenen Körperempfindungen gespeichert werden. Es sind diese vielschichtigen körperlichen Signale, die (gesunden) 
Menschen dazu verhelfen, sich stetig in der Welt zu orientieren und angemessene Entscheidungen zu fällen - ohne stetig alles bewusst zu analysieren: ${ }^{7}$

Gedanken und Gefühle sind keine neuen und selbstständigen Prozesse, die unabhängig von viszeralen Aktivitäten verlaufen; wir fühlen und denken aus dem Bauch heraus. [...] Weniger schmeichelhaft für unsere Ichbezogenheit, konzentriert sich diese (r)evolutionäre , von unten nach oben'-Sicht auf eine archaische, homöostatische Überlebensfunktion, welche die Vorlage für die neuronale Organisation und das Bewusstsein liefert. Unsere sogenannten höheren Gedankenabläufe, in die wir inzwischen so vernarrt sind, sind also eher Diener als Herren. (Levine/Petersen 2010: 311)

Man kann daher vermuten, dass Körperempfindungen auch beim (fremdsprachlichen) Schreiben wichtige Orientierungs- und Bewertungsgrundlagen liefern. Bei schreibbezogenen Problemen sind zum Beispiel ein ungutes Gefühl im Magen oder leichte Spannung in der Brust oder im Kopf denkbar. Das Auffinden einer passenden Formulierung könnte z. B. durch generelle (angenehme) muskuläre und viszerale Entspannung angezeigt werden. Der große Vorteil eines solchen felt sense (vgl. Gendlin 2008) ist, dass nicht alles kleinschrittig bewusst analysiert werden muss. Aus informellen Gesprächen mit (dafür sensibilisierten) SchreiberInnen weiß ich, dass die o.g. Körperempfindungen durchaus eine alltägliche Erfahrung beim Schreiben sind. Diese emotionalen und empfindungsbezogenen Verarbeitungsmechanismen sowie der potentiell bewusste $\mathrm{Zu}$ gang zu ihnen sind übrigens m. E. zwei wichtige Unterschiede zwischen Mensch und Maschine (interessante biologisch-psychologische Überlegungen zum Aufbau, zur Funktion und zur Entwicklung des menschlichen Bewusstseins finden sich ebenfalls bei Levine/Petersen 2010). In den von mir erhobenen Lautdenkprotokollen ließen sich jedoch keine Verbalisierungen finden, die über eine Steuerungsfunktion von Körperempfindungen beim Schreiben Auskunft geben. Es ist deshalb davon auszugehen, dass auch diese inneren Verarbeitungsmechanismen zum größten Teil automatisiert - und u. U. parallel zu Gedanken verlaufen und deshalb nicht verbalisiert werden. Trotz der Schwierigkeit, diese emotionalen und emotional-körperlichen Verarbeitungsmechanismen zu untersuchen, halte ich es für äußerst wichtig, derartige Überlegungen in die Diskussion zum (fremdsprachlichen) Schreiben mit einzubeziehen. Es kann vermutet werden, dass sich SchreiberInnen deutlich hinsichtlich ihrer schreibbezogenen emotionalen und empfindungsbezogenen Wahrnehmungs-, Aneignungs-, Steuerungs- und Regulationsmechanismen differenzieren und diese große Auswirkungen auf das schreibstrategische Vorgehen haben. Zudem ist anzunehmen, dass auch (fremd-) sprachliche Konzepte in Verbindung mit subtilen Körpersignalen verarbeitet und reaktiviert werden und dass sich auch FremdsprachenlernerInnen nicht unwesentlich dadurch unterscheiden, wie sehr sie Zugang zu ihrem empfindungsbezogenen Sprachgefühl haben. Erstaunlich ist deshalb,

\footnotetext{
${ }^{7}$ Levines Theorie stützt sich übrigens explizit auf das bereits 1981 von dem Psychologen Gendlin entwickelte Konzept des felt sense (vgl. z. B. Gendlin 1981, 1998).
} 
dass sich im Rahmen der Schreibforschung nur sehr wenige Forschende mit emotionalen Prozessen beschäftigt haben (einige der wenigen Ausnahmen sind neben Ortner 2000 z. B. Hayes/Nash 1996, Zimmerman/Kitsantas 2007). In gängigen Schreibprozessmodellen (z. B. de Beaugrande 1984, Bereiter/Scardamalia 1987, Hayes/Flower 1980, Kellog 1994, Molitor 1987, Oerter/Montada 1982, Wrobel 2003) sowie Modellen zum fremdsprachlichen Schreiben (vgl. Börner 1987, Chenoweth/Hayes 2001, Grabe 2001, Krings 1992, PortmannTselikas 1991) spielen emotionale und empfindungsbezogene Prozesse keine oder nur eine äußerst marginale Rolle. Ausführliche Kritik an diesem zweckrationalen Menschenbild der Schreibprozessforschung wurde übrigens ebenfalls von Ortner (2000) geäußert. Deutliche Einwände gegen das mentalistische Sprachverständnis der Fremdsprachenforschung hat zudem Schwerdtfeger (2000) erhoben (siehe auch Küster 2004).

Hinsichtlich emotionaler Verarbeitungsmechanismen kann also festgehalten werden, dass ein großer Einfluss auf die Abläufe beim (fremdsprachlichen) Schreiben vermutet werden kann. Obwohl in Lautdenkprotokollen zum fremdsprachlichen Schreiben vereinzelt Spuren zu emotionalen Verarbeitungsmechanismen erkannt werden können - teilweise übrigens deutlich auch über paralinguale Merkmale - bleibt doch die genaue Beschaffenheit der emotionalen Verarbeitung weitestgehend verborgen.

\subsection{Sozial-kommunikative Prozesse beim (fremdsprachlichen) Schreiben}

Im Gegensatz dazu, dass es den meisten Schreibmodellen an einer Modellierung der emotionalen Einflüsse auf das Schreiben fehlt, ist die große Bedeutung der sozial-kommunikativen und handlungsorientierten Aspekte des Schreibens häufiger diskutiert worden (vgl. z. B. Augst/Faigel 1986, Bereiter/Scardamalia 1987, Feilke/Augst 1989, Kruse 2006, Pohl 2007, Steinhoff 2007, Wrobel 1995) In Lautdenkprotokollen sind allerdings auf direktem Weg kaum Hinweise auf sozial-interaktive und kommunikative Prozesse zu finden. Einige der wenigen Beispiele, die ich in meinem Korpus finden konnte, sind im Folgenden dargestellt (das , $X^{\prime}$ steht anstelle des Namens eines/r der DozentInnen der sprachpraktischen Veranstaltungen, an denen die Studierenden zum Untersuchungszeitpunkt teilnahmen):

12. [...] ok das stimmt jetz so grammatisch aber versteht natürlich kein Schwein was ich damit sagen will deshalb jetzt noch mal ein SCHÖ:NES Beispiel nech wahr X [...] (InformantIn A ft2)

13. [...] genau das hat doch $\mathrm{X}$ gestern gezeigt dass da immer ein Komma hinkommt nach den ähm den Dingens $((1,2 \mathrm{~s}))$ articulateurs logiques $[\ldots]$ (InformantIn B ft2) 
14. [...] oh da würde $\mathrm{X}$ jetzt aber meckern mit dem ähm/ $((1,8 \mathrm{~s}))$ [löscht: la question pourquoi] weg damit [...] (InformantIn C ft2)

In Beispiel 12 realisiert die Informantin, dass ein/e LeserIn ihren soeben verschriftlichten Gedanken nicht verstehen kann. Sie kommt auch gleich auf die Lösung des Problems, nämlich zur Verdeutlichung ein Beispiel zu geben: „,nech wahr X“. Es kann vermutet werden, dass sich die Informantin an die Dozentin erinnert, die einige Tage zuvor im Rahmen der Schreibkurse die Herstellung einer guten Argumentationsstruktur in einem Text erklärt hat. In Beispiel 13 geht es zwar lediglich um eine Interpunktionsregel, aber auch an dieser Stelle ist eine mentale Simulation der Unterrichtssituation zu vermuten. In Beispiel 14 übernimmt die Informantin ganz offensichtlich die LeserInnen-Perspektive der Dozentin, wodurch eine Korrektur einer Formulierung erfolgt. Derartige Passagen sind allerdings - wie bereits erwähnt - in den von mir erhobenen Lautdenkprotokollen äußerst selten. Auch an dieser Stelle stoßen die Einblicksmöglichkeiten des Lauten Denkens an eine empfindliche Grenze, da nach allem, was insbesondere aus der Schreibentwicklungsforschung bekannt ist, die Perspektivübernahme eines/einer potentiellen Lesers/Leserin ein steter Bestandteil des (erwachsenen) Schreibens sein dürfte, in den Lautdenkprotokollen jedoch kaum zu finden ist. Ein sprach- bzw. diskursanalytischer Zugang könnte m. E. auch hinsichtlich dieser Phänomene eine interessante Alternative sein.

\subsection{Schreibspezifische Einblicksmöglichkeiten}

Nachdem nun einige vermutlich vorhandene innere Verarbeitungsmechanismen genannt wurden, die sich in Lautdenkdaten nicht direkt bzw. nur sehr selten rekonstruieren lassen, soll am Ende dieses Abschnitts noch einmal anhand eines Beispiels darauf eingegangen werden, welche schreibspezifischen Einblicke durch das Laute Denken im Rahmen einer fremdsprachlichen Textproduktion ermöglicht werden. Dabei kann jedoch lediglich eine exemplarisch-deskriptive Betrachtung vorgenommen werden. Welche Phänomene untersucht werden und welche Analyseebene in einer Untersuchung zum fremdsprachlichen Schreiben angestrebt wird, hängt von den Forschungsfragen sowie von den Bezugsmodellen und -theorien $a b$. Diese müssen sehr genau beschrieben und diskutiert werden und können potentiell ganz unterschiedlicher Natur sein (vgl. dazu auch z. B. Chi 1997, Heine/Schramm 2007, Kussmaul/Trikkonen-Condit 1995, Yang 2003). Zudem muss in einer zusammenhängenden Forschungsarbeit detailliert beschrieben und diskutiert werden, anhand welcher Phänomene in den Lautdenkprotokollen auf welche inneren Verarbeitungsweisen geschlossen wird. Dieses kann im Rahmen des im Folgenden präsentierten und diskutierten Beispiels nicht geleistet werden.

Zahlreiche Einblicke erhält man beim fremdsprachlichen Schreiben in Formulierungstätigkeiten sowie in das Verhalten beim Lösen von Problemen, die durch 
fremdsprachliche Kompetenzdefizite ausgelöst werden. Einige, allerdings weniger gute Einsichten werden im Bereich des Planungs- und Leseverhaltens sowie hinsichtlich der Evaluations- und Entscheidungstätigkeiten ermöglicht:

15. [...] genau dann schreib ich jetzt noch was zu den Eltern dass die halt immer da sind $((1,1 \mathrm{~s}))$ obwohl die sind ja vielleicht auch nicht bei allen da aber so im Allgemeinen halt en général $((1,1 \mathrm{~s}))$ man kann ja auch nicht auf jeden Einzelfall jetzt so ähm hä’ nee so $((1,1 \mathrm{~s}))$ äh eingehen $((1,2 \mathrm{~s}))$ les parents sont en général présents $((1,2 \mathrm{~s})) \mathrm{mh}$ na ja also so für die Wäsche und ja wie ähm ja so Haushaltsdinge insgesamt halt $((1,3 \mathrm{~s}))$ les choses ménagères vielleicht? $((1,1 \mathrm{~s}))$ oder ja ja les parents s'occupent des choses menagères das is gut $((1,1 \mathrm{~s}))$ les parents $((2,3 \mathrm{~s}))$ s'occupent $((1,2 \mathrm{~s}))$ mit $E N T$ ? ja is ja les parents ähm en général [Les parents s'occupent en généale $(\mathrm{Tk})]((1,2 \mathrm{~s}))$ aber das sind ja auch nich nur die choses ménagères sondern so das Administrative auch $((2,1 \mathrm{~s}))$ les choses administratives $((1,1 \mathrm{~s}))$ aber da ist dann ja der Haushalt wieder nicht drin $((2,5 \mathrm{~s}))$ Lebensdings Lebens/ $((1,3 \mathrm{~s}))$ ((gereizte Stimme)) wie heißt denn das?* Lebensbelange Lebenshaltungsbelange? $((2,3 \mathrm{~s}))$ nja vielleicht ähm Lebens/ $((3,1 \mathrm{~s}))$ oder genau Bedürfnisse kann man auch sagen $((3,4 \mathrm{~s}))\left[\mathrm{HSW}^{8}\right.$ : Bedürfnisse] ja genau les besoins das war Bedürfnisse $((1,1 \mathrm{~s}))$ nach Zuwendung ((unverständlich))* zu (?speziell) hier habm was doch ((laut und freudig)) les besoins essentiels Bedürfnisse des täglichen Lebens ha!* parfait $((1,3 \mathrm{~s}))$ dödödöm dependent de leurs professeurs ich hab den articulateur logique ganz vergessen $((1,2 \mathrm{~s}))$ ähm en plus vielleicht $((1,1 \mathrm{~s}))$ en plus [En plus] und dann mit il y a? ähm $((3,1 \mathrm{~s}))$ nee hatt ich nicht $((1,2 \mathrm{~s}))$ en plus il y a $((1,1 \mathrm{~s}))$ [il $y$ a $]$ und dann $((2,1 \mathrm{~s}))[L(e s)=>l(e s)]$ qui $((1,1 \mathrm{~s}))$ [qui] s'occupent en général oder ähm en plus en général les parents nee lass ich so des besoins essentiels $((1,9 \mathrm{~s}))$ oder de tous besoins? oder/ is das denn weiblich? toutes besoins' $((1,1 \mathrm{~s}))$ ich lass das hört sich so oder so komisch an des leurs mit s weils ja wieder zweimal Plural ist und dann enfants $((1,8 \mathrm{~s}))$ [des besoins essentiels de leurs enfants.] [...] (InformantIn B ft3)

Anhand dieses längeren Ausschnitts aus einem Lautdenkprotokoll kann man gut nachvollziehen, in welchen Schritten der Satz De plus il y a les parents qui $s$ 'occupent des besoins essentiels de leurs enfants entstanden ist. In einem ersten Schritt bringt die Informantin die Idee hervor, ,jetzt noch was zu [der Rolle] der Eltern [im Hinblick auf das Schülerleben]" zu schreiben. Das inhaltliche Problem, dass man die Aussage ,Eltern sind für ihre Kinder immer da' eigentlich nicht verallgemeinern sollte, löst die Informantin relativ schnell durch die textsortenbezogene Regel: „Ich schreibe im Allgemeinen“. Dafür findet sie auch gleich den passenden französischen Ausdruck en général. Die Informantin versucht diesen Gedanken auch gleich in einem ersten Formulierungsversuch umzusetzen. Dabei merkt sie jedoch, dass ihr Gedanke noch gar nicht ganz ausgereift ist, und entwickelt ihre Idee zunächst in der L1 weiter. Interessant dabei ist, dass das erste in diesem Rahmen aufkommende Problem gar kein fremdsprachenspezifisches ist, sondern in einem gewissen Sinne ein onomasiologisches Problem darstellt, da die Informantin weder in der Mutter- noch in der Fremdsprache für das, was sie sagen möchte, einen Ausdruck findet. Diese Suche nach dem passenden Wort kann man bei der Informantin relativ häufig

\footnotetext{
${ }^{8} \mathrm{HSW}$ ist die Abkürzung für Hilfsmittel-Such-Wort.
} 
beobachten. Einige andere von mir untersuchte SchreiberInnen geben sich bei ähnlichen Problemen wesentlich weniger Mühe und begnügen sich an derartigen Stellen z. B. mit Aufzählungen oder weniger präzisen Ausdrücken. Die drei Pausen in dieser Passage werfen allerdings die Frage auf, ob nicht noch weitere Ausdrücke von der Informantin in Betracht gezogen, aber nicht verbalisiert werden. Eine weitere interessante Beobachtung lässt sich in dem Beispiel zudem im Anschluss an die Wörterbuchbenutzung machen. Der Informantin fällt ein, dass sie „den articulateur logique [Konnektor] ganz vergessen“ hat. Diese Aussage löst die Vermutung aus, dass die Informantin das Kohäsions-Prinzip ,Benutze immer Bindewörter' verfolgt - eine Vermutung, die übrigens anhand anderer Passagen ihres Produktionsprozess noch untermauert werden kann. Eine Auffälligkeit, die sich ebenfalls auf die Textgrammatik bezieht, findet sich in dem Ausschnitt gleich im Anschluss. Die Informantin zieht in Erwägung, den Satz En plus, il y a des parents qui [...] zu schreiben, überprüft allerdings in der kurzen Pause, ob sie il y a nicht bereits in einem vorangegangenen Satz verwendet hat. Dieses lässt sich zum einen an der Verbalisierung „nee hatt ich nich“, zum anderen mithilfe des Bildschirmvideos erkennen, in welchem sichtbar wird, dass die Informantin während der Pause mit dem Cursor über die vorhergehenden Sätze fährt. Weitere aufschlussreiche Beobachtungen können zudem hinsichtlich fremdsprachbezogener Phänomene gemacht werden. Dabei sind die kurze Konjugationsunsicherheit hinsichtlich des Verbs occuper, die Frage nach dem Genus von besoins sowie die Angleichung von leur zu nennen. Ein weiteres fremdsprachenbezogenes Problem, bei welchem ein Wörterbuch konsultiert wird, ist die Frage nach dem Übersetzungsäquivalent zu „Bedürfnisse“. Dieses kann allerdings sehr schnell dank des Hilfsmittels gelöst werden. Durch die Verbalisierung, ,ja genau les besoins das war Bedürfnisse“ kann jedoch vermutet werden, dass die Informantin den Ausdruck bereits kannte. Bemerkenswert ist hinsichtlich der Wörterbuchbenutzung zudem, dass sich die Informantin den Wörterbucheintrag trotz des gefundenen Ausdrucks noch genauer anschaut. Dabei findet sie sehr schnell die Wendung „besoins essentiels - Bedürfnisse des täglichen Lebens", welche genau zu ihrem Ausdrucksbedürfnis passt. Die Strategie, sich bei der Wörterbuchbenutzung nicht mit dem ersten Übersetzungsäquivalent zu begnügen, sondern gewissenhaft den gesamten Eintrag auf feste Wendungen zu untersuchen, lässt sich übrigens ebenfalls in dem gesamten Lautdenkprotokoll der Informantin nachweisen. Dieses unterscheidet sie deutlich von einigen anderen von mir untersuchten studentischen SchreiberInnen, welche die Wörterbucheinträge nicht oder nur selten genauer anschauen.

Bereits anhand dieser kurzen Beschreibung wird deutlich, dass Lautdenkprotokolle reichhaltige Einblicke in das Verhalten eines Schreibers/einer Schreiberin ermöglichen. Die wichtigsten Fragen, die in diesem Rahmen beant- 
wortet werden können, sind u. a.: In welchen Teilschritten entstand der Text, ${ }^{9}$ welche Probleme traten dabei auf und wie wurden diese gelöst? Einer reinen Produktanalyse bleiben derartige Einblicke vollkommen verschlossen. Darüber hinaus können - in dem präsentierten Beispiel vergleichsweise gut - das einigen Bewertungen zugrundeliegende Konzeptionswissen bzw. das linguistische Makrostrukturwissen (Textgrammatik- und Kommunikationsnormen im weitesten Sinne) rekonstruiert werden. Zudem kommt auch in einigen Passagen das linguistische Mikrostrukturwissen sehr gut zur Geltung. Allerdings sind derartig gute Einblicke vergleichsweise selten. Bei einigen InformantInnen lassen sich z. B. ausschließlich Formulierungs- und Lesetätigkeiten rekonstruieren. Insbesondere hinsichtlich der textgrammatischen Planung besteht der begründete Verdacht, dass alle von mir untersuchten InformantInnen stetig abstrahieren und perspektivieren, ${ }^{10}$ diese Vorgänge allerdings nur dann rekonstruiert werden können, wenn es dabei zu größeren Schwierigkeiten kommt. Diese Vermutung resultiert zum einen daraus, dass in einigen Lautdenkprotokollen gar keine Verbalisierungen zu textgrammatischer Planung zu finden sind, obwohl die entstandenen Texte teilweise eine sehr gute Struktur aufweisen. Zum anderen lassen sich in meinem Korpus auch einige (retrospektive) Metakommentare finden, die ebenfalls diese Annahme untermauern:

16. [...] so noch mal die Aufgabenstellung $((1,1 \mathrm{~s}))$ wo ist sie denn ach $((2,3 \mathrm{~s}))$ genau d'un étudiant $((2,1 s))$ [étudiant] manchmal hab ich das Gefühl ich denke zwar ähm/ oder ich denke so schnell dass ichs gar nich so schnell aussprechen kann $((1,3 \mathrm{~s}))$ weil ich dann schon längst fertig gedacht habe und schon wieder was nächstes mache irgendwie naja $((2,1 \mathrm{~s}))$ das ist so ein Denken ohne Worte [...] (InformantIn B ft1)

17. [...] dölöm dölöm ähm $((5,1 \mathrm{~s}))$ nee kann so bleiben $((1,2 s))$ [professeurs.] gut sorry das ging gerade so schnell das konnte ich jetz nich alles sagen das waren ehr so Fetzen ob ich das doch noch anders anordne wegen $((1,4 \mathrm{~s}))$ ähm der Aufgabenstellung $((2,1 \mathrm{~s}))$ oder ob das noch passt so das Argument war aber irgendwie noch komplizierter glaub ich $((1,3 \mathrm{~s}))$ aber gut ich soll ja nichts erklären $((1,1 \mathrm{~s}))$ dann mal weiter $[\ldots]$ (InformantIn $\mathrm{G} \mathrm{ft1}$ )

18. [...] c'est pour cette raison que je vais discuter les côtés positives ja/ nur mal zur Info ich hab gerad überlegt ob ich da noch einerseits anderseits reinbringen sollte konnte ich aber nicht so schnell sagen passt aber auch nicht [...] (InformantIn F ft2)

Bei der textgrammatischen Planung scheint es sich also ebenfalls um Prozesse $\mathrm{zu}$ handeln, die bei den von mir untersuchten InformantInnen in großen Teilen vollkommen automatisiert verlaufen. Dieses ist nicht verwunderlich, da die

\footnotetext{
${ }^{9}$ Von Krings (1989) wurden übrigens drei interessante Datenanalysediagramme vorgeschlagen, mit deren Hilfe der Ablauf des (fremdsprachlichen) Schreibens systematisch dargestellt werden kann. Ausführlich werden sie in Krings (1989: 403f.) dargestellt.

${ }^{10}$ Interessante theoretische Überlegungen zum Prozess der Materialisierung von Gedanken bei der Sprachproduktion im Allgemeinen sowie für das Schreiben im Besonderen haben z. B. Chafe 1977, Molitor-Lübbert 2003, Ortner 1995, 2000, 2006 vorgelegt.
} 
jungen erwachsenen SchreiberInnen eine lange Schreibsozialisation durchlaufen haben. Damit bleibt jedoch in den meisten Fällen noch ein weiterer, für die Textproduktion konstitutiver innerer Vorgang verborgen - ein Umstand, der $\mathrm{m}$. E. in allen Untersuchungen zum (fremdsprachlichen) Schreiben dringend beachtet werden muss. Bei Profi-SchreiberInnen, bei denen aufgrund der hohen Automatisierung vieler Abläufe über Lautes Denken kaum Einblicke erwartet werden können, hat es sich übrigens bewährt, statt mit dem Verfahren des Lauten Denkens mit stimulated-recall-Interviews Daten zu elizitieren. Zwar erheben die ForscherInnen, die mit diesem Verfahren arbeiten, nicht den Anspruch, direkt mentale Aktivitäten der InformantInnen erfassen zu können, aber „es wird [immerhin] offen gelegt, was ein Autor oder eine Autorin sich prinzipiell überlegt haben könnte" (Hervorhebung i. O. Perrin 2001: 35). Wenn Erkenntnisse zum Schreibprozess von weit fortgeschrittenen SchreiberInnen erarbeitet werden sollen, ist dieses Vorgehen m. E. zu empfehlen (zu stimulatedrecall-Interviews bzw. videobasiertem Lauten Erinnern siehe auch Feick in diesem Band und Knorr in diesem Band).

An den Beispielen 16, 17 und 18 lässt sich zudem noch eine weitere interessante Überlegung, die $\mathrm{m}$. W. bisher nicht in der Fachliteratur diskutiert wurde, besprechen. Wenn InformantInnen gerade lesen, formulieren etc. - in der Terminologie von Ericsson/Simon 1993 ausgedrückt: level-1-Verbalisierungen produzieren (siehe Abschnitt 2) - könnte es u. U. zu Interferenzen mit level-2Verbalisierungen kommen. ${ }^{11}$ Insbesondere in Beispiel 18 liegt die Vermutung nahe, dass der Informant den retrospektiv geäußerten Gedanken nicht parallel aussprechen konnte, da sich seine Verbalisierungen gerade auf das Lesen bezogen. Aber auch in den anderen beiden Beispielen $(16,17)$ äußern die Informanten immer nach sprachbezogenen Tätigkeiten (Lesen und Schreiben), dass sie ihre Gedanken nicht verbalisieren konnten. Auch hinsichtlich empfindungsbezogener Prozesse wurde in Abschnitt 3.2 bereits erwähnt, dass diese wahrscheinlich nicht häufiger verbalisiert werden, da sie vermutlich zeitgleich mit anderen Prozessen auftreten. Es wäre sehr wünschenswert, dass auch hinsichtlich dieser Phänomene methodenbezogene Untersuchungen durchgeführt werden.

Auch wenn aus Platzgründen an dieser Stelle nicht ausführlich auf die Auswertung von Lautdenkprotokollen eingegangen werden kann, möchte ich dennoch mit Blick auf die dargebotenen Beispiele eine kurze grundsätzliche Einschätzung zur Analyse von Lautdenkdaten vornehmen. Die präsentierten Ausschnitte aus den Lautdenkprotokollen und die in diesem Rahmen getätigten Interpretationen zeigen m. E. deutlich, dass bei der Auswertung von Lautdenkprotokollen im hohen Maße auch Kontextwissen einbezogen werden sollte. Insbesondere in Beispiel 15 konnten wiederkehrende (schreib)strategische Verhaltensweisen der Informantin nur über die Einbeziehung des Wissens aus der

${ }^{11}$ Für diesen Hinweis danke ich Dr. Thora Tenbrink (Universität Bremen). 
Gesamtanalyse des Textproduktionsprozesses erkannt werden. Aber auch im Rahmen der anderen, in diesem Aufsatz getätigten Analysen der Lautdenkdaten war es nötig, dass ich mich so gut wie möglich in die InformantInnen hineinversetzte, um das Verhalten im Rahmen der Gesamtsituation zu interpretieren. Eine solche Analysehaltung widerspricht teilweise der von Ericsson/Simon (1993) vorgeschlagenen - und bis heute häufig eingesetzten - Auswertungsstrategie, zunächst die Lautdenkprotokolle zu segmentieren und anschließend im Rahmen einer Zufallsauswahl den Segmenten eine Kategorie zuzuweisen. Durch die hier präsentierten Beispiele konnte $m$. E. jedoch gezeigt werden, dass es die besonders fragmentarische Qualität von Lautdenkdaten zwar nötig macht, fundierte Kriterien für die Kodierung von Lautdenkdaten zu entwickeln, aber dass es ebenso wichtig ist, dass sich der/die AnalysatorIn mit viel Kontextwissen und Intuition in die Handlungen, Gefühle und Gedanken der InformantInnen hineinversetzt ${ }^{12}$ (vgl. dazu auch Yang 2003 sowie Krings 1994: 296).

\section{Zusammenfassung und Perspektiven}

Es konnte verdeutlicht werden, dass sich über Lautdenkdaten, die im Rahmen einer fremdsprachlichen Textproduktion entstanden sind, zahlreiche grundlegende sowie sprach- und schreibspezifische innere Verarbeitungsmechanismen vermutlich nicht oder nur sehr bedingt freilegen lassen. Diese Grenzen sollten bei jeder Forschungsarbeit bedacht, ausführlich diskutiert und - soweit dieses möglich ist - durch den Einsatz komplementärer Erhebungsverfahren kompensiert werden. Weitere methodenbezogene Studien wären in Anbetracht des Paradigmenwechsels in der Kognitiven Psychologie zudem sehr wünschenswert. Außerdem sollte m. E. in nächster Zeit auch die Rolle der analysierenden Person von Lautdenkprotokollen erneut diskutiert und definiert werden (siehe dazu auch Smagorinsky 2001, Yang 2003) - insbesondere dann, wenn in Zukunft auch emotionale, empfindungsbezogene, situationale und soziale Prozesse stärker in die Datenanalyse mit einbezogen werden. Wenig genutzt wurde zudem bisher die Möglichkeit, sprach- bzw. diskursanalytische Verfahren in Verbindung mit dem Lauten Denken einzusetzen - eine Herangehensweise, die ebenfalls vielversprechende Untersuchungsperspektiven eröffnen könnte. Trotz der Tatsache, dass durch Lautes Denken vermutlich nur begrenzte Einblicke in innere Repräsentations- und Verarbeitungsmechanismen ermöglicht werden, halte ich den Einsatz des Verfahrens, ebenso wie Aguado (2004), dennoch für gewinnbringend:

Es ist unbestritten, dass ein Großteil der kognitiven Verarbeitung und des emotionalen

Erlebens unbewusst verläuft; dies darf jedoch kein Argument dafür sein, dass diejenigen

\footnotetext{
${ }^{12}$ Weitere Hinweise zur Auswertung von Lautdenkdaten finden sich u. a. bei Chi 1997, Heine 2010, Heine/Schramm 2007, Krings 1986, 1994, Kussmaul 1992 und Yang 2003.
} 
Zustände und Prozesse, die bewusstseinsfähig und somit verbalisierbar sind [...] nicht mittels introspektiver Verfahren untersucht werden sollten. (Aguado 2004: 32)

Abschließend möchte ich erwähnen, dass es insgesamt wünschenswert wäre, wenn den emotionalen, empfindungsbezogenen, situationalen und sozialen Prozessen sowohl in der (fremdsprachlichen) Schreibprozessforschung als auch in der Sprachlehr- und -lernforschung deutlich mehr Aufmerksamkeit geschenkt würde. Wie in diesem Aufsatz gezeigt werden konnte, wirken sie wahrscheinlich stärker auf das gesamte menschliche Erleben ein, als bislang angenommen bzw. in vielen Untersuchungen berücksichtigt wurde.

Ich danke Prof. Dr. Hans Peter Krings, Prof. Dr. Lena Heine, Prof. Dr. Susanne Göpferich, Prof. Dr. Karen Schramm, Dr. Thora Tenbrink, Linn Gralla und Evelyn Bergman herzlich für die vielen hilfreichen Kommentare und Anregungen zu meinem Aufsatz.

\section{Literatur}

Aguado, Karin (2004). Introspektive Verfahren in der empirischen Fremdsprachenerwerbsforschung. Methodisch-methodologische Überlegungen. Fremdsprachen und Hochschule 71: 24-38.

Augst, Gerhard / Faigel, Peter / Müller, Karin / Feilke, Helmuth (1986). Von der Reihung zur Gestaltung. Untersuchungen zur Ontogenese der schriftsprachlichen Fähigkeiten von 1323 Jahren. Frankfurt am Main: Lang.

Barsalou, Lawrence W. (2008). Grounded Cognition. Annual Review of Psychology 59: 617645.

Beare, Sophie / Bourdages, Johanne S. (2007). Skilled writers' generating strategies in L1 and L2. In: Torrance, Mark et al. (eds.): Writing and cognition. Research and applications. Amsterdam. Elsevier. 1. ed. 151-162.

Beaugrande, Robert de (1984). Text production. Toward a science of composition. Norwood, N.J.: Ablex Publ. Corp.

Bereiter, Carl / Scardamalia, Marlene (1987). The psychology of written composition. Hillsdale, NJ: Erlbaum.

Betsch, Tilmann / Funke, Joachim / Plessner, Henning (2011). Denken - Urteilen, Entscheiden, Problemlösen. Berlin: Springer-Verlag.

Boeckx, Cedric (2010). Language in cognition. Uncovering mental structures and the rules behind them. Malden, Mass: Wiley-Blackwell.

Börner, Wolfgang (1987). Schreiben im Fremdsprachenunterricht: Überlegungen zu einem Modell. In: Lörscher, Wolfgang / Hüllen, Werner (eds.): Perspectives on language in performance. Studies in linguistics, literary criticism and language teaching and learning; to honour Werner Hüllen on the occasion of his 60. birthday. Tübingen: Narr. 1336-1349. 
Bowles, Melissa A. (2010). The think-aloud controversy in second language research. New York: Routledge.

Caron, Jean / Caron-Prague, Josiane (1986). Towards a psycholinguistic approach of argumentative operators: The 'thinking aloud' procedure. In: Van Eemeren, Frans H. / Grootendorst, Rob / Blair, J. Anthony / Willard, Charles A. (eds.): Argumentation: Perspectives and approaches: Proceedings of the Conference on Argumentation. Dordrecht, Holland: Foris Publications. 170-178.

Chafe, Wallace L. (1977). Creativity in verbalization and its implications for the nature of stored knowledge. In R.O. Freedle (ed.): Discourse production and comprehension. Norwood, NJ. Ablex Publ. Corp. 41-55.

Chenoweth N. Ann / Hayes, John R. (2001): Fluency in writing. Generating text in L1 and L2. In: Written Communication 18/1. 80-98.

Chi, Michelene T. H. (1997). Quantifying qualitative analyses of verbal data: A practical guide. Journal of the Learning Sciences 6/3: 271-315.

Cohen, Andrew D. (1998). Strategies in learning and using a second language. Harlow, Essex:Longman..

Damasio, Antonio R. (2006). Descartes' Irrtum. Fühlen, Denken und das menschliche Gehirn. München: List. 4. Aufl.

Damasio, Antonio R. / Kober, Hainer (2007). Der Spinoza-Effekt. Wie Gefühle unser Leben bestimmen. Berlin: List. 4. Aufl.

Damasio, Antonio R. / Kober, Hainer (2009). Ich fühle, also bin ich. Die Entschlüsselung des Bewusstseins. München: List. 8. Aufl.

Ericsson, K. / Simon, H. (1993). Protocol analysis. Verbal reports as data. Cambridge, MA.: MIT Press.

Feilke, Helmuth / Augst, Gerjard (1989). Zur Ontogenese der Schreibkompetenz. In: Antos, Gerd / Krings, Hans P. (Hrsg.): Textproduktion. Ein interdisziplinärer Forschungsüberblick. Tübingen: Niemeyer. 297-327.

Gendlin, Eugene T. (1981). Focusing. Technik der Selbsthilfe bei der Lösung persönlicher Probleme. Salzburg: Müller.

Gendlin, Eugene T. (1998): Focusing. Selbsthilfe bei der Lösung persönlicher Probleme. Reinbek bei Hamburg: Rowohlt-Taschenbuch-Verl.

Gendron, Maria / Lindquist, Kristen A. / Barsalou, Lawrence / Barrett, Lisa F. (2012). Emotion words shape emotion percepts. Emotion 12/2: 314-325.

Göpferich, Susanne (2008). Translationsprozessforschung. Stand, Methoden, Perspektiven. Tübingen: Narr.

Grabe, William (2001): Notes toward a theory of second language writing. In: Silva, Tony J. / Matsuda, Paul K. (Hrsg.): On second language writing. Mahwah NJ. L. Erlbaum Associates. 39-57.

Hayes, John R / Flower, Linda S. (1980). Identifying the organization of writing processes. In: Gregg, Lee W. (ed): Cognitive processes in writing. Hillsdale, NJ. Erlbaum. 3-30. 
Hayes, John .R. / Nash. Jane G (1996). On the nature of planning in writing. In: Levy, M. C. / Ransdell S. (eds.): The science of writing. theories, methods, individual differences, and applications New York: Erlbaum. 29-56.

Heine, Lena (2005). Lautes Denken als Forschungsinstrument in der Fremdsprachenforschung. Zeitschrift für Fremdsprachenforschung 16/2: 163-185.

Heine, Lena (2010). Problem solving in a foreign language. Berlin: De Gruyter Mouton.

Heine, Lena / Schramm, Karen (2007). Lautes Denken in der Fremdsprachenforschung: Eine Handreichung für die empirische Praxis. In: Vollmer, Helmut Johannes (Hrsg.): Synergieeffekte in der Fremdsprachenforschung. Empirische Zugänge, Probleme, Ergebnisse. Frankfurt am Main: Peter Lang. 167-206.

Kellogg, Ronald T. (1994). The psychology of writing. New York: Oxford University Press.

Krings, Hans P. (1986). Was in den Köpfen von Übersetzern vorgeht. Eine empirische Untersuchung zur Struktur des Übersetzungsprozesses an fortgeschrittenen Französischlernern. Tübingen: Narr.

Krings, Hans P. (1989). Schreiben in der Fremdsprache - Prozeßanalyse zum ,vierten skill'. In: Antos, Gerd / Krings, Hans P. (Hrsg.): Textproduktion. Ein interdisziplinärer Forschungsüberblick. Tübingen. Niemeyer. 377-436.

Krings, Hans P. (1992). Schwarze Spuren auf weißem Grund- Fragen, Methoden und Ergebnisse der Schreibprozeßforschung im Überblick. In: Krings, Hans P. / Antos, Gerd (Hrsg.): Textproduktion. Neue Wege der Forschung. Trier: WVT Wissenschaftlicher Verlag. 45-110.

Krings, Hans P. (1994). Texte reparieren. Empirische Untersuchungen zum Prozeß der Nachredaktion von Maschinenübersetzungen. Habilitationsschrift. Universität Hildesheim

Kruse, Otto / Berger, Katja / Ulmi, Marianne (Hrsg.) (2006). Prozessorientierte Schreibdidaktik. Schreibtraining für Schule, Studium und Beruf. Bern: Haupt Verlag.

Kussmaul, Paul / Trikkonen-Condit, Sonja (1995). Think-aloud protocol analysis in translation studies. TTR-Traduction Terminologie Redaction 8/1: 177-199.

Küster, Lutz (2004). Emotion und Kognition im Bildungsgedanken. Erziehungswissenschaftlich-soziologische Aspekte eines interkulturellen Fremdsprachenunterrichts. Fremdsprachen und Hochschule 71/1: 7-23.

Levelt, Willem J. M. / Roelofs, Ardi / Meyer, Antje S. (1999). A theory of lexical access in speech production. Behavioural and Brain Science 22: 1-75.

Levine, Peter A. / Petersen, Karin (2011). Sprache ohne Worte. Wie unser Körper Trauma verarbeitet und uns in die innere Balance zurückführt. München: Kösel. 2. Aufl.

Levy, C. M. (ed.) (1996). The science of writing. Theories, methods, individual differences, and application. Mahwah, NJ: Lawrence Erlbaum.

Molitor, Sylvie (1987). Weiterentwicklung eines Textproduktionsmodells durch Fallstudien. Unterrichtswissenschaft 15/4. 396-409.

Molitor-Lübbert, Sylvie (2003). Schreiben und Denken. Kognitive Grundlagen des Schreibens. In: Perrin, Daniel (Hrsg.): Schreiben. Von intuitiven zu professionellen Schreibstrategien. Wiesbaden: Westdeutscher Verlag, 2. Aufl. 33-47. 
Oerter, Rolf / Montada, Leo (1982). Entwicklungspsychologie. Ein Lehrbuch. München: Urban \& Schwarzenberg.

Ortner, Hanspeter (1995). Die Sprache als Produktivkraft. Das (epistemisch-heuristische) Schreiben aus der Sicht der Piagetschen Kognitionspsychologie. In: Baurmann, Jürgen / Weingarten, Rüdiger (Hrsg.): Schreiben. Prozesse, Prozeduren und Produkte. Opladen: Westdeutscher Verlag. 320-364.

Ortner, Hanspeter (2000). Schreiben und Denken. Tübingen: Niemeyer.

Ortner, Hanspeter (2003a). Schreiben und Wissen. Einfälle fördern und Aufmerksamkeit staffeln. In: Perrin, Daniel (Hrsg.): Schreiben. Von intuitiven zu professionellen Schreibstrategien. Wiesbaden. Westdeutscher Verlag. 2. Aufl. 63-81.

Ortner, Hanspeter (2003b). Synkretismus statt Gestaltung - ein Problem beim wissenschaftlichen Schreiben. In: Ehlich, Konrad / Steets, Angelika (Hrsg.): Wissenschaftlich schreiben - lehren und lernen. Berlin: de Gruyter. 186-210.

Ortner, Hanspeter (2006). Spontanschreiben und elaboriertes Schreiben - wenn die ursprüngliche Lösung zu einem Teil des (neuen) Problems wird. In: Berning, Johannes / Keßler, Nicola / Koch, Helmut H. (Hrsg.): Schreiben im Kontext von Schule, Universität, Beruf und Lebensalltag. Berlin. Lit-Verlag. 77-101.

Ortner, Hanspeter (2007). Die (schriftliche) Darstellung von Sachverhalten als Stimulus für die Denk-, Sprach- und Schreibentwicklung. In: Schmölzer-Eibinger, Sabine u. a. (Hrsg.): Textkompetenz. Eine Schlüsselkompetenz und ihre Vermittlung. Tübingen: Narr. 113-139.

Panksepp, Jaak (2004). Basic Affects and the Instinctual Emotional Systems of the Brain. In: Manstead, Antony S. R. (eds.): Feelings and emotions. The Amsterdam Symposium. Cambridge Univ. Press. 174-193.

Perrin, Daniel (2001). Wie Journalisten schreiben. Ergebnisse angewandter Schreibprozessforschung; inklusive CD-ROM mit Fallstudien aus Presse, Radio, TV und Internet. Konstanz: UVK-Verlag.-Ges.

Pohl, Thorsten (2007). Studien zur Ontogenese wissenschaftlichen Schreibens. Tübingen: Niemeyer.

Portmann-Tselikas, Paul R. (1991). Schreiben und Lernen. Grundlagen der fremdsprachlichen Schreibdidaktik. Tübingen: Niemeyer.

Rickheit, Gert / Weiss, Sabine / Eikmeyer, Hans-Jürgen (2010). Kognitive Linguistik. Theorien, Modelle, Methoden. Tübingen: Francke.

Roth, Gerhard (2004). Aus Sicht des Gehirns. Frankfurt am Main: Suhrkamp.

Roth, Gerhard (2009). Fühlen, Denken, Handeln. Wie das Gehirn unser Verhalten steuert. Frankfurt am Main: Suhrkamp.

Sasaki, Tomomi (2008). Concurrent think aloud protocol as a socially situated construct. IRAL: International Review of Applied Linguistics in Language Teaching 46/4: 349-374.

Schnell, Anna K. (in Vorbereitung). Arbeitstitel: Untersuchung zur Entwicklung des fremdsprachlichen Schreibverhaltens von Studierenden. Dissertationsprojekt. Universität Bremen.

Schwarz, Monika (2008). Einführung in die kognitive Linguistik. Tübingen: Francke. 3. Aufl.

Schwarz-Friesel, Monika (2007). Sprache und Emotion. Tübingen: Francke. 
Schwerdtfeger, Inge C. (2000). Leiblichkeit und Grammatik. In: Düwell, Henning (Hrsg.): Dimensionen der didaktischen Grammatik. Festschrift für Günther Zimmermann zum 65. Geburtstag. Bochum: AKS-Verlag. 281-303.

Shapiro, Lawrence A. (2011). Embodied cognition. London: Routledge.

Sinha, Chris (2010). Cognitive Linguistics, Psychology and Cognitive Science. In: Geeraerts, Dirk (ed.): The Oxford handbook of cognitive linguistics. Oxford Univ. Press. 1266-1294.

Smagorinsky, Peter (2001). Rethinking protocol analysis from a cultural perspective. Annual Review of Applied Linguistics 21: 233-245.

Steinhoff, Torsten (2007). Wissenschaftliche Textkompetenz. Sprachgebrauch und Schreibentwicklung in wissenschaftlichen Texten von Studenten und Experten. Tübingen: Niemeyer.

Swain, Merrill (2006). Verbal protocols: what does it mean for research to use speaking as a data collection tool? In: Chalhoub-Deville, Micheline et al. (eds.): Inference and generalizability in applied linguistics. Multiple perspectives. Amsterdam: Benjamins. 97-113.

Tenbrink, Thora (2010): Kognitive Diskursanalyse. In: Stolz, Thomas / Ruigendijk, Esther / Trabant, Jürgen (Hrsg.): Linguistik im Nordwesten: Beiträge zum 1. Nordwestdeutschen Linguistischen Kolloquium. Bochum: Brockmeyer. 117-133.

Wrobel, Arne (1995). Schreiben als Handlung. Überlegungen und Untersuchungen zur Theorie der Textproduktion. Tübingen: Niemeyer.

Wrobel, Arne (2003). Schreiben und Formulieren. Prätext als Problemindikator und Lösung. In: Perrin, Daniel u. a. (Hrsg.): Schreiben. Von intuitiven zu professionellen Schreibstrategien. Wiesbaden: Westdeutscher Verlag. 2. Aufl. 83-96.

Yang, Ching S. (2003). Reconceptualising think-aloud methodology: Refining the encoding and categorizing techniques via contextualized perspectives. Computers in Human Behavior 19/1: 95-115.

Zimmerman, Barry J. / Kitsantas, Anastasia (2007). A writer's disciplin: The development of self-regulatory skill. In: Hidi, Suzanne / Boscolo, Pietro (eds.): Writing and motivation. Amsterdam: Elsevier. 51-69. 



\section{Themenbereich Qualitative Inhaltsanalyse}





\title{
Die Qualitative Inhaltsanalyse in der empirischen Fremdsprachenforschung: Grenzen, Potentiale, Desiderata
}

\section{Karin Aguado}

\begin{abstract}
The aims of the present article are the following: first of all, I will give an overview over the typical features of Qualitative Content Analysis (QIA). After a brief discussion of the possible reasons why the QIA is becoming increasingly attractive to German SLA researchers, I will describe recent methodological developments and illustrate them with a number of selected studies. Then, I will present some preliminary ideas for a revised model of QIA which integrates deductive and inductive steps of category formation in order to improve the analysis process. Finally, I will propose a few topics to be addressed in future methodological discussions of the QIA, especially in terms of empirical SLA studies.
\end{abstract}

\section{Einleitung}

Insgesamt ist festzustellen, dass die deutschsprachige empirische Fremdsprachenforschung bevorzugt qualitativ arbeitet - ein Trend, der trotz anhaltender Polemik hinsichtlich der (Un-)Wissenschaftlichkeit, der vermeintlichen Subjektivität oder der mangelnden Generalisierbarkeit qualitativer Studien seit geraumer Zeit ungebrochen ist. Ein möglicher Grund für diese Entwicklung könnte sein, dass mit dem steigenden Interesse der Fremdsprachenlehr- und -lernforschung und der Fremdsprachendidaktik an der Innensicht der an Lehr- und Lernprozessen Beteiligten seit den 1990er Jahren immer häufiger Untersuchungen dieser Prozesse und/oder der darin involvierten Faktoren wie z. B. Erfahrungen, Einstellungen, Emotionen oder (Meta-)Kognitionen durchgeführt werden. Zu den am häufigsten verwendeten Verfahren zur Erhebung solcher subjektiven Daten zählen qualitative, vorwiegend halbstrukturierte Interviews, Gruppendiskussionen, (halb-)offene Fragebögen, Tagebuch- oder Logbuchaufzeichnungen, Sprachlernbiographien, Lautdenkprotokolle oder auch retrospektive Gespräche zu konkreten Lehr-Lern-Ereignissen. Damit sollen in erster Linie nicht-beobachtbare affektive oder kognitive Zustände oder Vorgänge ermittelt, nachvollzogen und im Idealfall auch erklärt werden, u. a. um dann auf einer anwendungsbezogenen Ebene angemessene Empfehlungen sowie Maßnahmen zur Optimierung von fremdsprachlichen Lehr- und Lernprozessen zu entwickeln.

Für die Auswertung von qualitativen Daten bieten sich - je nach Forschungsgegenstand und Erkenntnisinteresse - unterschiedliche Methoden an wie z. B. verschiedene diskurs- und konversationsanalytische Ansätze, die Objektive Hermeneutik (Oevermann et al. 1979), die Grounded Theory (Glaser/Strauss 1967/ 1998), das Forschungsprogramm Subjektive Theorien (Groeben et al. 1988) 
oder die im Folgenden näher betrachtete QIA (vgl. dazu auch die Beiträge in Flick et al. 2000). Sämtliche Verfahren weisen Vor- und Nachteile auf, die bei der Auswahl und ggf. Kombination hinsichtlich ihrer Gegenstandsangemessenheit zu reflektieren und gegeneinander abzuwägen sind.

Während die bei der Erhebung von qualitativen Daten zu beachtenden Kriterien und Vorgaben in der forschungsmethodischen Literatur insgesamt gut beschrieben sind (vgl. z. B. Bortz/Döring 2006 oder Lamnek 2005), stehen genaue Darstellungen zur Vorgehensweise bei der qualitativen Analyse von Daten noch weitgehend aus. So wundert es nicht, dass in Bezug auf diese Phase des Forschungsprozesses große methodische Unsicherheiten bestehen und dass insbesondere mit der qualitativen Auswertung noch nicht vertraute Studierende oder Nachwuchswissenschaftler/innen, aber auch erfahrenere L2-Forscher/innen nicht ohne Weiteres einschätzen können, welches Verfahren für welche Zwecke am besten geeignet, d. h. gegenstandsangemessen ist, wie die genaue Vorgehensweise zu sein hat und was dabei zu beachten ist. Die unbefriedigende Literaturlage in Bezug auf die Analysepraxis beginnt sich jedoch allmählich zu bessern. So liegt seit kurzem mit dem von Kuckartz (2012) verfassten Lehrbuch Qualitative Inhaltsanalyse. Methoden, Praxis, Computerunterstützung eine erste praktische Anleitung zur systematischen und methodisch kontrollierten Anwendung der QIA vor, die gewiss von großem Nutzen für Forscherinnen und Forscher verschiedenster Disziplinen und Qualifikationsstufen ist.

Mit der Bezeichnung, Qualitative Inhaltsanalyse verbindet man in der deutschsprachigen Methodendiskussion in erster Linie die von Philipp Mayring im Jahr 1982 erstmals im Detail beschriebene und seither kontinuierlich weiterentwickelte Variante der klassischen, d. h. quantitativ orientierten Variante der Inhaltsanalyse. Der Quantifizierung sind im Laufe der Zeit unterschiedliche Stellenwerte beigemessen worden. So schreibt Mayring im Jahr 2000: „Dabei will sich die qualitative Inhaltsanalyse quantitativen Analyseschritten nicht verschließen, sondern versucht sie in den Analyseablauf begründet einzubeziehen“" (Mayring 2000: 471). In der aktuellen (elften!) Auflage seines Grundlagenwerks formuliert er seine Auffassung demgegenüber wie folgt: „Der grundlegende Ansatz der qualitativen Inhaltsanalyse ist nun, die Stärken der quantitativen Inhaltsanalyse beizubehalten und auf ihrem Hintergrund Verfahren systematischer qualitativ orientierter Textanalyse zu entwickeln" (Mayring 2010: 48). Da die QIA zunehmend auch im Rahmen von explorativ-interpretativ angelegten Forschungsprojekten eine Rolle spielt, soll im Anschluss an die Skizzierung ihrer Entstehungsgeschichte sowie ihrer wesentlichen Merkmale auf die in der einschlägigen Literatur formulierte Kritik eingegangen werden, bevor das Potential der QIA für die qualitative Fremdsprachenforschung diskutiert wird. 


\section{Zur Entwicklung der QIA: ein kurzer Abriss ${ }^{1}$}

Die Entwicklung der QIA in ihrer heutigen Version begann in den 1920er Jahren in den USA, als große massenmediale Textdatenmengen systematisch quantitativ - d. h. hinsichtlich Häufigkeit, Intensität und Kontingenz ausgewählter Items - ausgewertet werden sollten. Durch die Allerton House Conference, die im Jahr 1959 an der University of Illinois stattfand, erhielt sie einen wichtigen methodischen Schub, und zwar v. a. dadurch, dass neben der Zusammenfassung des Inhalts auf der deskriptiven Ebene fortan auch das Ableiten von Schlussfolgerungen für wichtig erachtet wurde, und zwar sowohl hinsichtlich der Entstehung als auch hinsichtlich der Wirkung der untersuchten Inhalte. Zusätzlich zu den o. g. quantitativen Parametern rückten im Laufe der Zeit zunehmend qualitative Bedeutungen in den Fokus der methodisch-methodologischen Diskussion. Die im Jahr 1966 an der University of Pennsylvania durchgeführte Annenberg School Conference fokussierte schließlich vorwiegend praktische Aspekte der Inhaltsanalyse bzw. des Analyseprozesses. Außerdem kam es zur überfälligen - Forderung nach der Explizierung des einer Auswertung jeweils zugrundeliegenden Kommunikationsmodells, was einen weiteren Schritt in Richtung einer qualitativen Fundierung bedeutete (vgl. dazu v. a. Gerbner et al. 1969 sowie Krippendorf 1969). Zusammenfassend ist festzustellen, dass es im Laufe der Zeit insgesamt zu einer allmählichen Annäherung zwischen qualitativen und quantitativen Herangehensweisen kam. In Bezug auf die Anregung Kracauers (1952) - nämlich nicht nur vordergründige, an der sprachlichen Oberfläche manifeste, sondern auch latente Sinnstrukturen zu ermitteln und zu analysieren - bis zu Mayrings (1982) Abfassung des ersten systematisch und methodisch begründeten Lehrbuchs zum Zweck der praktischen Analysearbeit sollten jedoch noch etwa 30 Jahre vergehen.

Bei den drei Grundformen der QIA - nämlich Zusammenfassung, Explizierung und (inhaltliche, skalierende sowie typisierende) Strukturierung - handelt es sich laut Mayring um „drei voneinander unabhängige Analysetechniken, die nicht als nacheinander zu durchlaufende Schritte verstanden werden sollen. Vielmehr gilt es, je nach Forschungsfrage und Material die geeignete Analysetechnik auszuwählen“ (Mayring 2010: 65). ${ }^{2}$

Eine klassische quantitative Inhaltsanalyse verläuft strikt linear und umfasst die folgenden Schritte: 1. Formulierung des Erkenntnisinteresses, Aufstellung von Hypothesen, Definition einer Grundgesamtheit der zu analysierenden Texte,

\footnotetext{
${ }^{1}$ Einen guten historischen Überblick über die Entstehungs- und Entwicklungsgeschichte der QIA bietet z. B. Mayring (2010: 26-47).

${ }^{2}$ Für eine ausführliche Darstellung der hier knapp skizzierten Formen und ihrer Spezifika siehe Mayring (2010: 48-109); vgl. dazu auch die Beiträge von Eberhardt, Gläser-Zikuda und Rogge in diesem Band.
} 
2. Entwicklung bzw. Definition eines Kategoriensystems, Formulierung von Kodierregeln, 3. Durchführung eines Kodiertrainings, 4. Durchführung der Kodierung und 5. statistische Auswertung. Ein zentraler Unterschied zu einer qualitativen Inhaltsanalyse, der bereits an dieser Stelle offenkundig wird, besteht darin, dass es sich bei letzterer um einen iterativen und damit vertieften, aber entsprechend aufwendigen und langsamen Prozess handelt. Typisch für qualitative Forschungen ist überdies, dass die einzelnen Forschungsphasen nicht so strikt voneinander getrennt sind wie in quantitativen Studien. Während z. B. nachträgliche Modifizierungen in quantitativ angelegten Untersuchungen als Zeichen unzureichender Planung und Kontrolle gewertet werden, sind Ergänzungen, Differenzierungen und Verfeinerungen im qualitativen Forschungsprozess (vgl. dazu auch Kuckartz 2012: 51f.) nichts Unerwünschtes, sondern werden eher als Ausdruck des Primats der Daten gewertet.

Welches der genannten Analyseverfahren zu wählen ist, ist also von seiner Eignung abhängig, die jeweilige(n) Forschungsfrage(n) beantworten zu können. Das folgende von Mayring angestellte Gedankenexperiment hinsichtlich der grundsätzlichen Möglichkeiten, unbekanntes Material zu analysieren, soll die Vorgehensweise veranschaulichen:

Man stelle sich vor, auf einer Wanderung plötzlich vor einem gigantischen Felsbrocken [...] zu stehen. Ich möchte wissen, was ich da vor mir habe. Wie kann ich dabei vorgehen? Zunächst würde ich zurücktreten, auf eine nahe Anhöhe steigen, von wo ich einen Überblick über den Felsbrocken bekomme. Aus der Entfernung sehe ich nicht mehr die Details, aber ich habe das „Ding“ als Ganzes in groben Umrissen im Blickfeld, praktisch in einer verkleinerten Form (Zusammenfassung). Dann würde ich wieder herantreten und mir bestimmte besonders interessant erscheinende Stücke genauer ansehen. Ich würde mir einzelne Teile herausbrechen und untersuchen (Explikation). Schließlich würde ich versuchen, den Felsbrocken aufzubrechen, um einen Eindruck von seiner inneren Struktur zu bekommen. Ich würde versuchen, einzelne Bestandteile zu erkennen, den Brocken zu vermessen, seine Größe, seine Härte, sein Gewicht durch verschiedene Messoperationen festzustellen (Strukturierung). (Mayring 2010: 56)

Anhand dieser Beschreibung wird deutlich, dass die Reihenfolge, mit der die einzelnen Formen der QIA angewendet werden können, nicht beliebig ist. Im Gegenteil: Zwar müssen sie nicht zwingend miteinander kombiniert werden, aber falls eine Kombination beabsichtigt ist, sollte die Zusammenfassung sinnvollerweise zu Beginn des Analyseprozesses durchgeführt werden. Hinsichtlich der Explikation besteht theoretisch die Möglichkeit, sie vor oder nach der Strukturierung einzusetzen - mit unterschiedlichen Auswirkungen auf die Analyse und die damit erzielbaren Erkenntnisse. Das von Mayring (2010) aufgestellte allgemeine Ablaufmodell der QIA sieht folgendermaßen aus: 


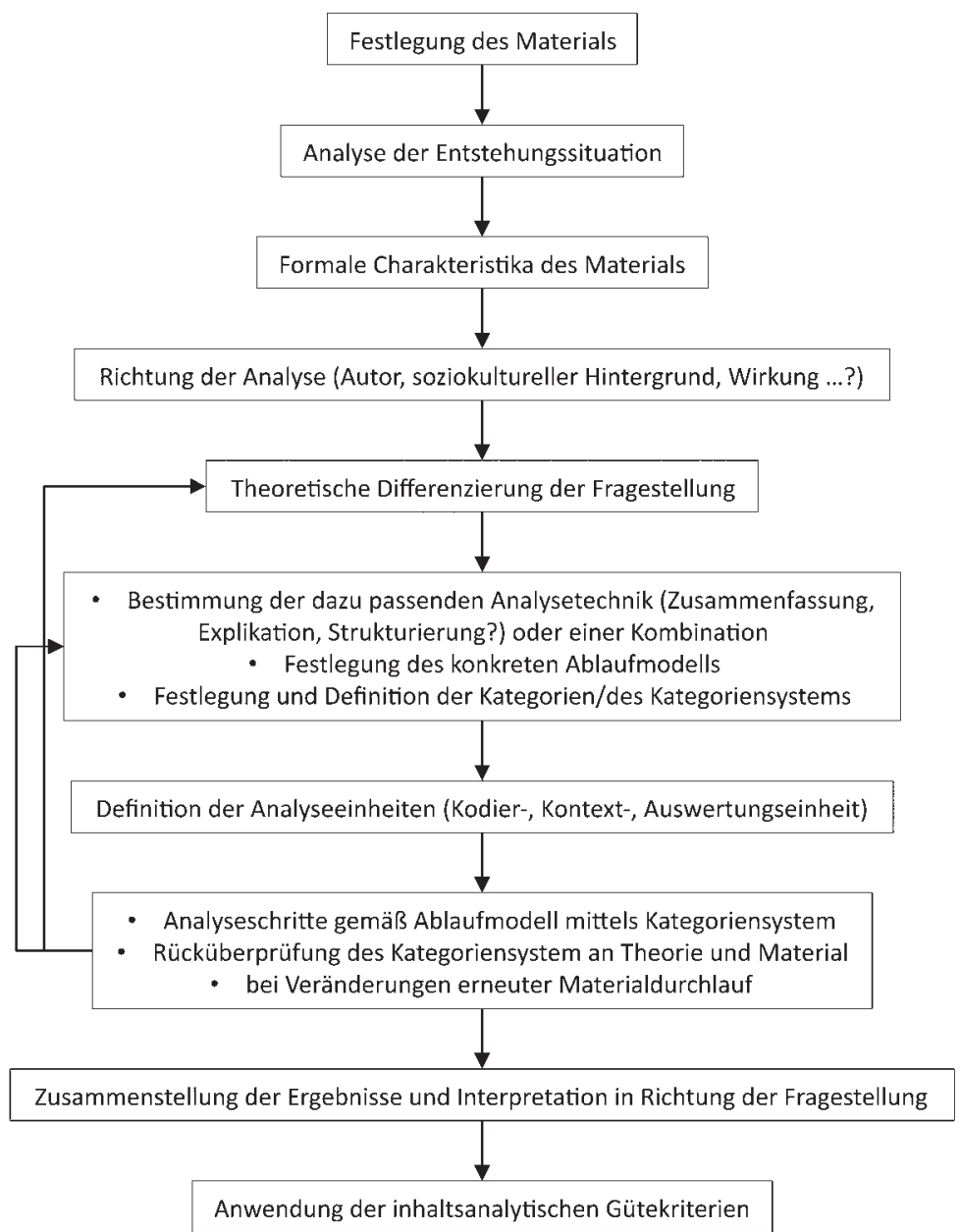

Abbildung 1: Allgemeines inhaltsanalytisches Ablaufmodell (nach Mayring 2010: 60)

Im Vorgriff auf den nun folgenden Abschnitt sei darauf hingewiesen, dass die QIA den für die qualitative Forschung typischen Kriterien wie Transparenz, Offenheit oder Flexibilität prinzipiell gerecht wird. Neben einer gewissen Dynamik, wie sie in den Teilschritten „Rücküberprüfung des Kategoriensystems an Theorie und Material“ und „bei Veränderungen erneuter Materialdurchlauf“ (s. o.) sichtbar wird, ist die Möglichkeit einer eher theorie- oder einer eher empiriegeleiteten Vorgehensweise oder einer Kombination von beidem im allgemeinen inhaltsanalytischen Ablaufmodell (siehe die Schritte „Bestimmung der dazu passenden Analysetechnik [Zusammenfassung, Explikation, Struktu- 
rierung?] oder einer Kombination...“ und „Analyse gemäß Ablaufmodell mittels Kategoriensystem...") grundsätzlich angelegt.

\section{Merkmale verstehender Inhaltsanalysen und zentrale Prinzipien qualitativer Forschung}

Für die Auswertung von qualitativen Daten bieten sich - je nach Forschungsgegenstand und Erkenntnisinteresse - unterschiedliche Methoden an. Aus den Grundbedingungen hermeneutischer Verfahren (vgl. dazu Klafki 1971) leitet Kuckartz (2012: 33) eine Reihe von Handlungsregeln ab, die sich mit den im Folgenden skizzierten zentralen Gütekriterien der qualitativen Forschung in Einklang bringen lassen. Die Einhaltung solcher Kriterien ist ein grundlegendes Prinzip eines jeden Forschungsprozesses - sei er analytisch-nomologisch oder explorativ-interpretativ angelegt. Gleichzeitig besteht weitgehend Konsens darüber, dass die sogenannten klassischen, im Rahmen naturwissenschaftlicher bzw. quantitativer Forschung entwickelten Gütekriterien Objektivität, Reliabilität, Validität und Generalisierbarkeit nicht oder nur bedingt auf qualitative Forschungen anwendbar sind. Aufgrund der generell begrenzten Standardisierung qualitativer Forschungen und der z. T. eigens entwickelten methodischen Instrumentarien und der daraus resultierenden eingeschränkten Replizierbarkeit und Überprüfbarkeit qualitativer empirischer Studien sowie aufgrund grundsätzlich kleinerer Fallzahlen mussten für die qualitative Forschung notwendigerweise andere Kriterien entwickelt werden. Dies hat in den vergangenen 15-20 Jahren zur Aufstellung einer Reihe von qualitativen Kriterienkatalogen geführt. Da jedoch je nach disziplinspezifischer Kultur unterschiedliche Schwerpunkte gesetzt werden, wird es möglicherweise nie einen allgemeingültigen Konsens (vgl. z. B. Lamnek 2005, Miles/Huberman 1994 oder Steinke 2000) geben. Die im Folgenden skizzierten Kriterien scheinen mir jedoch den kleinsten gemeinsamen Nenner zu bilden.

Die von Glaser \& Strauss (1967/1998) zu Beginn der methodisch-methodologischen Diskussion in den empirischen Sozialwissenschaften geforderte Unvoreingenommenheit einer Forscherin/eines Forschers ihrem/seinem Gegenstand gegenüber ist angesichts des heutigen Diskussionstands schlichtweg undenkbar: Spätestens seitdem konstruktivistische Überlegungen auch in die Methodendiskussion Einzug gehalten haben, wissen wir, dass jede Wahrnehmung zwingend vom jeweiligen Vorwissen und den daraus resultierenden Erwartungen geprägt ist bzw. konstruiert wird. Bei qualitativen Analysen ist das Verstehen sowohl die Voraussetzung als auch das Ziel der Interpretation von Texten - insbesondere wenn es um die (Re-)Konstruktion von latenten Sinnstrukturen und kommunikativen Absichten geht. Je mehr Vorwissen vorhanden ist, desto mehr und besser können wir verstehen bzw. begründete Hypothesen über die Bedeutung und Funktion von Texten aufstellen. Die im Rahmen empirischer Forschungen 
daraus zu ziehende methodische Konsequenz ist die maximale Offenlegung und Reflexion dieses den gesamten Forschungsprozess steuernden Vorwissens. Ebenso unverzichtbar ist die Benennung der Bedingungen, unter denen die zu analysierenden Daten entstanden sind. Für jegliche Weiterverarbeitung unabdingbar ist ferner eine genaue Beschreibung der Daten sowie die Entwicklung eines Gesamtverständnisses des zu analysierenden Inhalts. Im Falle von verbalen Daten sollte dies idealerweise vor der vollständigen Verschriftlichung vorhanden sein, weil Entscheidungen hinsichtlich des Feinheitsgrades der Transkription davon abhängig sind, inwiefern die erhobenen Daten Antworten auf die Forschungsfragen erwarten lassen. In dieser Phase geht es also um die Schärfung des Blicks für die sich anschließende Analyse der Daten. Zur angemessenen Analyse und Interpretation von verbalen Daten bedarf es einer systematischen, nachvollziehbaren Vorgehensweise. Die von Kuckartz (2012: 23) geforderte „Notwendigkeit methodischer Strenge“ ist unverzichtbar, wenn die erzielten Ergebnisse über die eigene peer group hinaus anerkannt und akzeptiert werden sollen.

Zusammenfassend lässt sich also festhalten, dass im qualitativen Forschungsprozess jede Phase (Vorbereitung, Durchführung, Aufbereitung, Auswertung) zwecks Transparenz und intersubjektiver Nachvollziehbarkeit genau und lückenlos zu dokumentieren ist, um der ,für jede qualitative Studie einmaligen Dynamik zwischen Gegenstand, Fragestellung und methodischem Konzept Rechnung" (Steinke 2000: 324) zu tragen. Ein weiteres, hiermit unmittelbar verknüpftes Kriterium ist das der Offenheit bzw. der Flexibilität sowohl dem Gegenstand als auch dem methodischen Vorgehen gegenüber. Dabei geht es darum, „den Wahrnehmungstrichter" [...] so weit wie möglich offen zu halten, um auch unerwartete und dadurch instruktive Informationen zu erhalten" (Lamnek 2005: 21). Im Unterschied zu den aufgrund standardisierter Erhebungs-, Aufbereitungs- und Analyseverfahren vergleichsweise leichter auszuwertenden quantitativen Daten sind qualitative Daten insgesamt weniger vorstrukturiert. Daher verlangen sie auf Seiten der Forschenden grundsätzlich einen höheren Grad an Offenheit und Flexibilität sowohl in Bezug auf deren Erhebung als auch hinsichtlich ihrer Auswertung. Von besonderer Bedeutung ist daher das Kriterium der Gegenstandsangemessenheit der zu wählenden methodischen Vorgehensweise.

Abschließend sei festgestellt, dass die QIA für Untersuchungen im Kontext des Lehrens und Lernens von Fremdsprachen geeignet erscheint, da sie hinsichtlich ihrer Vorgehensweise - insbesondere im Vergleich zur klassischen Inhaltsanalyse - prinzipiell auch für eher explorative, induktive, kategorienbildende Verfahren bzw. für eine Kombination verschiedener methodischer Herangehensweisen offen ist. 


\section{Zur Kritik an der Qualitativen Inhaltsanalyse}

Ein wesentliches Ziel der QIA liegt in der systematischen Reduktion von Komplexität. $\mathrm{Zu}$ diesem Zweck werden Texte an der sprachlichen Oberfläche nach theoretisch abgeleiteten, deduktiven Kategorien durchsucht, klassifizierend bearbeitet und schließlich quantitativ ausgewertet. Eine zentrale Kritik an der QIA betrifft gerade den Aspekt der Quantifizierung und die damit verbundene analytisch-segmentierende Vorgehensweise und deren Konsequenzen für die Interpretation von Texten. In Bezug auf die Faktoren ,Bedeutung ' und ,Kontext' gehen wir spätestens seit Wittgenstein (1953/1977: PU §43) davon aus, dass ein Wort an sich keine Bedeutung hat, sondern diese erst durch seinen Gebrauch erhält. Dasselbe gilt für einen Text: Auch er steht immer in einem sprachlichen und situativen Kontext, der miterfasst und gedeutet werden muss, will man die Bedeutung eines Textes angemessen verstehen. Angesichts des "schrittweisen, den Text in einzelne Analyseeinheiten zergliedernden, an Kategorien(-systemen) orientierten Vorgehen“ (Mayring 2000: 471), wie es die QIA vorsieht, lässt sich fragen, ob in einer den Kontext im Wesentlichen außer Acht lassenden Schrittfür-Schritt-Analyse überhaupt latente Sinnstrukturen entdeckt werden können. Auf den Punkt gebracht lautet die Kritik hier: Ein Kategoriensystem an das Datenmaterial heranzutragen, wie Mayring (2010: 92) es vorschlägt, ist keine qualitative, sondern eine ausschließlich überprüfende und somit quantitative Vorgehensweise, mittels derer nur das gefunden werden kann, was gesucht wird bzw. was zuvor bereits bekannt ist.

Aus qualitativer Sicht besonders problematisch ist hierbei die Vernachlässigung der Ganzheitlichkeit und der Spezifik des Einzelfalls. So meint auch Lamnek (2005: 518): „Der Einzelfall wird eben doch zu einer Sammlung von Merkmalsausprägungen“, d. h. zu einer quantitativ auswertbaren Menge. Hierbei ist zu beachten, dass zum einen Frequenz nicht mit Relevanz gleichzusetzen ist und dass zum anderen zwischen den Zeilen stehende latente Sinnstrukturen nicht durch wortbasierte quantifizierende Verfahren erfasst werden können, sondern dass dafür der sprachliche und meist auch der nicht-sprachliche Kontext einzubeziehen ist. Es wird also kritisiert, dass das ganzheitliche, tiefe Verstehen eines Textes durch die zu frühe Anwendung von zuvor festgelegten Kategorien erschwert wird und eine voreilige Entfernung vom Text mit sich bringt.

Eine spezifische Kritik an der zusammenfassenden Inhaltsanalyse (vgl. Mayring 2010: 69, siehe Abbildung 2) lautet, dass der Hauptfokus auf den propositionalen Gehalt des Textes gelegt wird. Insbesondere bei mündlichen Daten sind jedoch nicht-propositionale Kontextualisierungshinweise wie Pausen, Intonation, Betonung, aber auch Modalpartikeln oder andere einstellungsbezogene Ausdrücke wie z. B. „eigentlich“, „etwa“, „,vielleicht“ für ein angemessenes Verstehen und Nachvollziehen von Inhalten höchst relevant. Durch die Ausblendung solcher konnotativen Bedeutungen entsteht $\mathrm{u}$. U. ein verzerrtes Bild, 
da es gerade diese Ausdrucksmittel sind, die Hinweise auf latente Sinnstrukturen liefern können und steuernd auf die Interpretation durch den Rezipienten wirken.

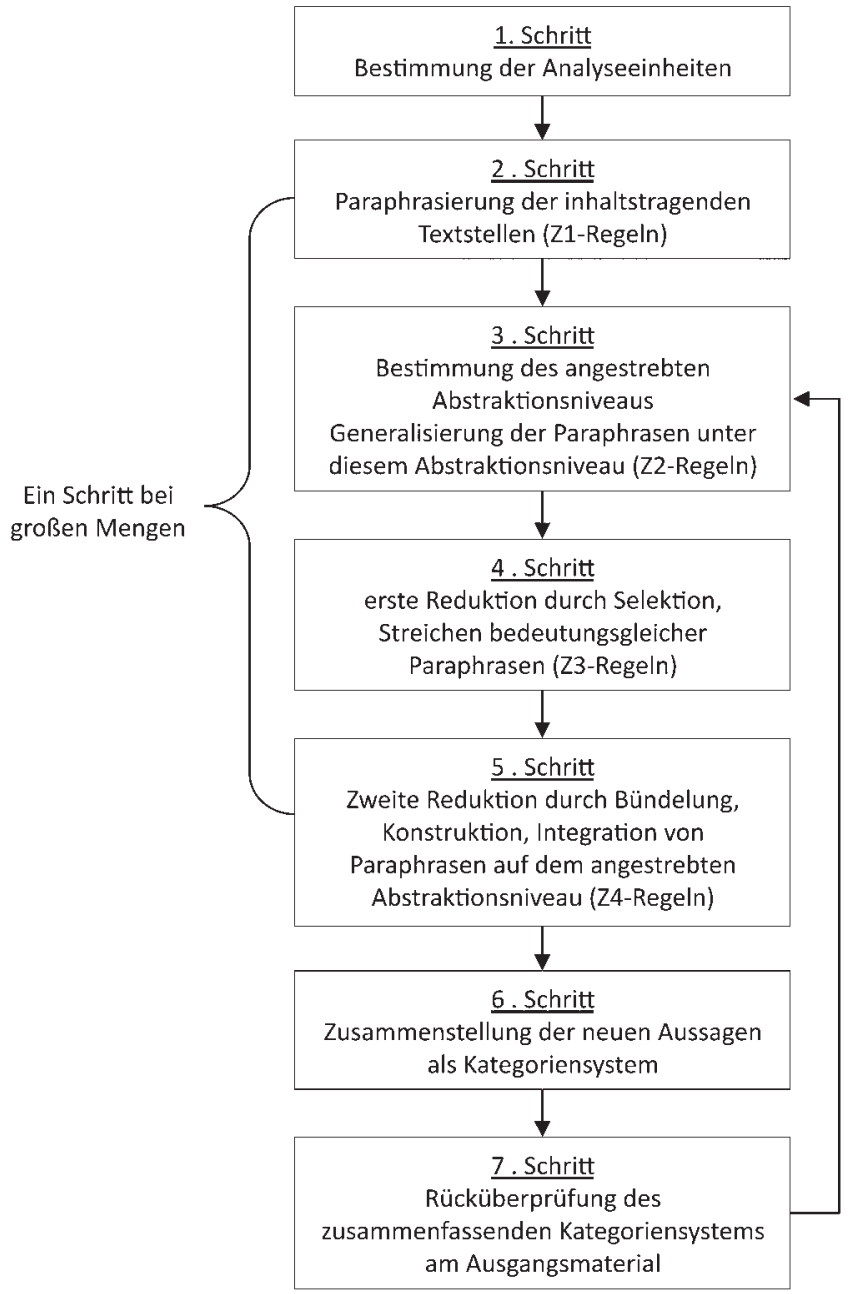

Abbildung 2: Ablaufmodell zusammenfassender Inhaltsanalyse (nach Mayring 2010: 68)

Ein weiterer - eher auf einer Meta-Ebene angesiedelter - Kritikpunkt an der strukturierenden qualitativen Inhaltsanalyse, der sehr ausführlich von Steigleder (2008) formuliert wurde, betrifft den Schritt 7 des folgenden Ablaufmodells von Mayring (2010). 


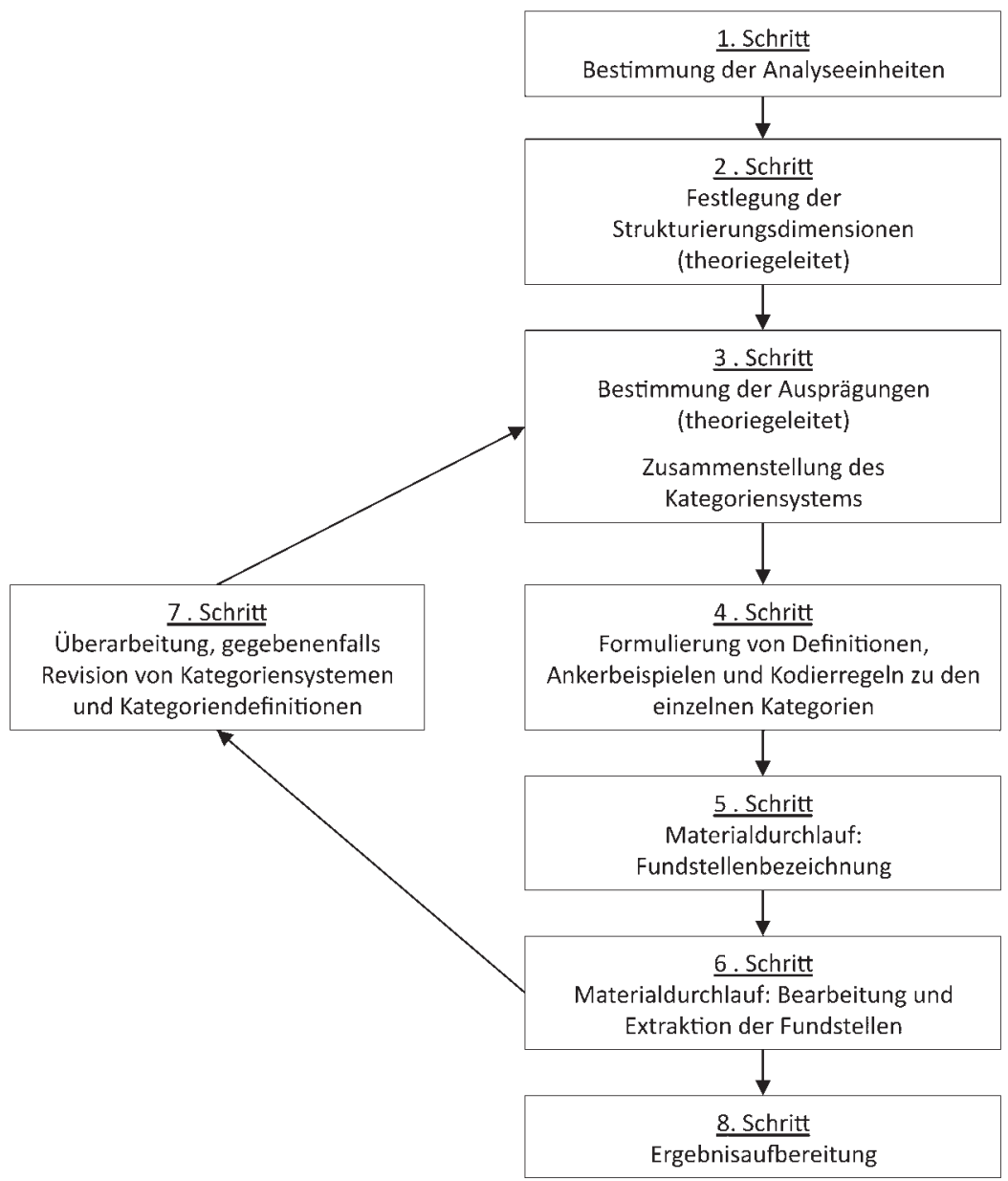

Abbildung 3: Ablaufmodell strukturierender Inhaltsanalyse (allgemein) (nach Mayring 2010: 93)

Der Vorwurf lautet, dass die „sehr offen gehaltene Revisionsoption im Auswertungsprozess eine folgenschwere Fehlerquelle für die Auswertung des gesamten Datenmaterials“ (Steigleder 2008: 180) darstelle. Was den Ausführungen von Steigleder zufolge bei diesem Analyseschritt fehlt, sind Informationen zu den möglichen Gründen, die eine Überarbeitung erforderlich machen. So bleibe ungeklärt, was die Kriterien für unangemessen gewählte oder falsch definierte Kategorien seien. Des Weiteren fehlten konkrete Handlungsanweisungen dazu, wie eine Überarbeitung zu erfolgen habe. Steigleder kritisiert die Uneindeutigkeit der von Mayring formulierten Revisionsanweisung, die insbesondere 
bei „Erstanwendern“ (Steigleder 2008: 181) zu Verunsicherungen oder Missverständnissen führen könne. Die Autorin fordert, dass die Prinzipien Systematik, Regelgeleitetheit und intersubjektive Nachprüfbarkeit nicht nur für den Forschungsprozess, sondern auch für die Beschreibung der Auswertungsmethodik und ihre Übertragung in die Forschungspraxis Gültigkeit haben sollten.

Zusammenfassend ist festzustellen, dass sich die von verschiedenen Seiten formulierte Kritik an der QIA letztlich auf wenige Punkte reduzieren lässt, denen aufgrund der prinzipiellen Offenheit dieser Analysemethode konstruktiv begegnet werden kann, wie im folgenden Abschnitt anhand von einigen Beispielen gezeigt werden soll.

\section{Zum Potential der QIA für die empirische Fremdsprachen- forschung}

Was ist an der QIA trotz der vielfach vorgebrachten und zuvor knapp skizzierten Kritik nun so attraktiv, dass sie in jüngster Zeit zunehmend insbesondere vom wissenschaftlichen Nachwuchs in der empirischen Fremdsprachenforschung angewendet wird, und wie sehen diese Anwendungen im Einzelnen aus?

Das besondere Potential der QIA für die empirische Fremdsprachenforschung liegt zunächst einmal darin, dass mit ihrer Hilfe komplexe Fragestellungen bearbeitet werden können. Das Lehren und Lernen von fremden Sprachen ist solch ein komplexer Prozess, der eines differenzierten und reflektierten methodischen Instrumentariums bedarf - insbesondere, wenn ein noch unerforschtes Themengebiet bearbeitet wird oder wenn vertiefte Einsichten in Deutungsprozesse individuell oder sozial geprägter Wirklichkeitskonstruktion gewonnen werden sollen. Zur Verarbeitung und Interpretation solcher Daten werden systematische, komplexitätsbewältigende und intersubjektiv überprüfbare Auswertungsverfahren benötigt.

Da es bei der qualitativen Auswertung von Daten um nachvollziehendes Verstehen und Rekonstruieren geht, für das Erfahrung, gesunder Menschenverstand, Empathie, die Fähigkeit zur Einnahme einer Fremdperspektive sowie eine hohe soziale und kommunikative Kompetenz erforderlich sind, kann es insbesondere bei Forschungsanfängern, deren Kompetenzen sich in den genannten Bereichen noch in der Entwickelung befinden - zu großen Unsicherheiten und Zweifeln kommen. D. h. jegliche Werkzeuge oder Handlungsanweisungen, die diesen Prozess unterstützen oder der Kompensation von nicht ausreichend vorhandener Erfahrung dienen, sind willkommen und werden gern angenommen. Was an der QIA dabei besonders geschätzt wird, ist ihre systematische, strukturierte und damit prinzipiell erlernbare Vorgehensweise. Zudem mag es an ihrer intuitiven Plausibilität sowie an ihrer Orientierung am alltäglichen Umgang mit sprachlichem Material und den damit verbundenen Pro- 
zessen des Verstehens und Interpretierens, des Zusammenfassens, des Explizierens und des Strukturierens sowie an der ihr inhärenten Flexibilität liegen, dass die QIA eine solche Anziehungskraft ausübt.

Die Ermittlung und Analyse impliziter Bedeutungen erfordert die Betrachtung des sprachlichen und situativen Kontextes. In diesem Zusammenhang stellt Eberhardt (in diesem Band) zu Recht fest, dass Kritiker der QIA übersehen,

\begin{abstract}
dass die indikatorbasierte Interpretation der qualitativen Inhaltsanalyse jedem Forscher an vielen Stellen des Ablaufmodells einen großen Spielraum lässt. Insbesondere die stets individuell zu treffenden Entscheidungen über die Größe der Kodier- und Kontexteinheiten sind dafür verantwortlich, wie weit sich die Dateninterpretation von der Denotation einzelner Wörter und der Textoberfläche entfernt und auch konnotative Wortbedeutungen und pragmalinguistische Aspekte in der Interpretation verbaler Aussagen miteinbezieht, inwiefern sie einzelne Sätze und Textpassagen nicht isoliert, sondern in ihrem Kontext betrachtet, und damit auch die Rekonstruktion latenter Sinneinheiten ermöglicht. (Eberhardt, in diesem Band)
\end{abstract}

Am Einsatz der QIA in der empirischen Fremdsprachenforschung ist besonders auffällig, dass es kaum eine Arbeit gibt, die das ursprünglich vorgeschlagene methodische Vorgehen unverändert übernimmt. Stattdessen werden in nahezu jeder Studie spezifische Anpassungen vorgenommen. ${ }^{3}$ Dazu zählt v. a. die Kombination von theorie- und empiriegeleiteter Kategorienbildung, die ,je nach Thematik bzw. Forschungsgegenstand sinnvoll sein [kann], um bestehende Theorien zu überprüfen, zu erweitern oder auch neue Theorien zu generieren“ (Steigleder 2008: 170; vgl. dazu auch Gläser/Laudel 2009: 199). Insbesondere für die Erfassung subjektiver Wahrnehmungen und Deutungsprozesse sowie für die Aufdeckung latenter Sinnstrukturen erscheint die Integration von deduktiver und induktiver Kategorienbildung attraktiv.

Jüngere empirische Studien zeichnen sich ferner dadurch aus, dass sie zwecks Validierung ihrer Ergebnisse oder zwecks Erweiterung von Erkenntnismöglichkeiten die QIA mit anderen Daten oder Methoden kombinieren. Ein Beispiel für die Anwendung einer solchen Triangulierungsstrategie liefert die Studie von Beier (im vorliegenden Band), die zwecks Erhöhung der Validität ihrer Studie die Prozesse der Rezeption von Bildern und Texten sowohl mittels (semi-) visueller Leseprotokolle als auch durch retrospektive Gespräche erfasst und miteinander abgleicht. Auch Eberhardt und Rogge (beide im vorliegenden Band) kombinieren verschiedene Vorgehensweisen und Verfahren miteinander, um auf diese Weise ihren Erkenntnisgewinn zu maximieren. Eberhardt trianguliert die von ihm erhobenen schriftlichen und mündlichen Daten (Beurteilung kulturell

\footnotetext{
${ }^{3}$ Die sich hier stellende Frage, ob und inwiefern solche Modifikationen legitim sind - also: darf eine Einzelforscherin/ein Einzelforscher eine Analysemethode modifizieren, ohne sie zu verfälschen und damit ihren ursprünglichen Zweck verändern? - kann aus Platzgründen an dieser Stelle nicht im Detail behandelt werden, wäre jedoch ganz sicher ein interessanter Gegenstand für eine transdisziplinäre methodologische Diskussion und somit ein Desiderat.
} 
aufgeladener Fotos und Kommentierung von Filmausschnitten mit interkulturellen Begegnungssituationen) von Französischlernenden der Klasse 10 zur Ausdifferenzierung und Weiterentwicklung eines Strukturmodells interkultureller Kompetenz. Dabei kombiniert er außerdem eine deduktive Kategoriendefinition zu den Teillernzielen interkultureller Kompetenz nach Byram (1997) mit induktiv aus seinen Daten abgeleiteten zusätzlichen Kategorien. Das Forschungsziel von Rogge besteht darin, einen vertieften Einblick in inter- bzw. transkulturelle Lernprozesse im Rahmen von fremdsprachlichen schulischen Begegnungsprojekten $\mathrm{zu}$ erhalten, um zu ermitteln, inwieweit solche Projekte zur Wahrnehmung interkultureller Differenzen in der Begegnung dienen und ob es durch die Begegnungssituation zugleich zu einer transkulturellen Bereicherung und zum Fremdverstehen kommen kann. Rogge nutzt für sein exploratives Vorgehen das von Steigleder (2008) entworfene alternative Modell einer theorie- und empiriegeleiteten Kategorienbildung (ebd.: 189), deren Vorteil er darin sieht, dass auf diese Weise nicht nur deduktive (d. h. aus der Theorie zum inter- und transkulturellen Lernen abgeleitete Kriterien) und induktive (d. h. konkret aus dem Datenmaterial extrapolierte Kriterien) Kategorien kombiniert werden können, sondern zugleich wesentliche Verfahrensschritte schon während des Auswertungsprozesses kontrolliert werden können. So angewendet ist die QIA weniger ein linearer als vielmehr ein iterativer und kommunikativer Dialog des Forschers mit dem eigenen Theorieverständnis und den eigenen Vorannahmen sowie mit dem konkret aus den Daten ermittelten empirischen Befund, der darüber hinaus mittels kommunikativer Validierungen mit den Interpretationen der Forschungsteilnehmer/innen abgeglichen werden kann.

Auch Haudeck (2005) arbeitet sowohl integriert deduktiv-induktiv als auch triangulierend, indem sie die schriftlich erhobenen Hauptdaten von Lernenden einer achten Realschulklasse, die ihre Vorkabellernstrategien beim Englischlernen in einem Lerntagebuch beschreiben, mit weiteren Daten wie Klassenarbeiten, Tests oder Konzeptionen von Abschlussprüfungen flankiert. Abschließend empfiehlt sie zusätzliche Introspektionen (wie z. B. Videoaufnahmen, gleichzeitiges Lautes Denken oder problemzentrierte Interviews), die u. a. den Vorteil mit sich brächten, „die zeitliche Distanz zwischen Lernaktivität und dem Sich-Erinnern“ (Haudeck 2005: 102) zu minimieren, als auch die Möglichkeit, dass Lernende sich mündlich äußern - was ihnen erfahrungsgemäß leichter fällt als sich schriftlich zu äußern - „und der Forscher die Möglichkeit hat, ad-hocFragen zu stellen, wenn es die Situation erfordert, z. B. wenn sich der Lernende ungenau ausdrückt oder wenn der Forscher Rückfragen zur subjektiven Perspektive der Lernenden hat" (ebd.).

Auch bezüglich des folgenden Aspekts ist festzustellen, dass empirische L2Forscher/innen in methodisch-methodologischer Hinsicht außerordentlich produktiv sind: Da sich die QIA in erster Linie auf ausgewählte, mittels inhaltlicher Kategorien analysierte, synchrone Produkte konzentriert und dabei die ins- 
besondere in Bezug auf den Bereich ,Lehren und Lernen' zentrale Perspektive der Prozesshaftigkeit praktisch nicht berücksichtigt, unternimmt Wilden (2006) einen ersten Versuch, mittels der QIA auch diachrone Entwicklungen zu erfassen. In ihrer einen Zeitraum von vier Monaten umfassenden Studie untersucht sie die Selbst- und Fremdwahrnehmungen von deutschen und britischen Fremdsprachenlehrer/innen im Rahmen eines interkulturellen Onlineaustauschs. Ziel der Studie ist neben der Ermittlung der Selbst- und Fremdbilder die Untersuchung der Frage, ob es Indizien für die Entstehung einer sogenannten ,Dritten Perspektive" gibt. Die Autorin erfasst die Prozesshaftigkeit dieser Perspektiventwicklung durch die induktive Analyse der Diskursverläufe der einzelnen Lehrer/innenpaare, rekonstruiert sie und untersucht sie hinsichtlich typischer oder vergleichbarer Verläufe.

Wie eine mögliche Modifikation des von Mayring entwickelten Verfahrens der QIA mit dem Ziel einer gleichberechtigten Berücksichtigung sowohl theoriegeleiteter als auch empirisch aus dem Datenmaterial entwickelter Kategorien aussehen könnte und wie zugleich die Balance zwischen größtmöglicher Offenheit gegenüber dem Forschungsgegenstand und der grundsätzlichen Regelgeleitetheit des Verfahrens realisiert werden könnte, kann - mit Bezug auf die Überlegungen von Haudeck (2005), Wilden (2006, 2008), Steigleder (2008), Gläser-Zikuda, Eberhardt, Rogge und Beier (alle in diesem Band) - aus Platzgründen hier nur angedeutet werden: Theoriegeleitet und auf der Basis des eigenen Vorverständnisses sowie gemäß des jeweiligen Erkenntnisinteresses gebildete Kategorien werden zunächst einmal auf ihr Vorkommen im Datenmaterial überprüft und ggf. verifiziert oder falsifiziert. Sie werden dann um die im Verlauf des weiteren Analyseprozesses zusätzlich aus dem Material empirisch abgeleiteten Kategorien ergänzt, mit ihnen abgeglichen und ggf. miteinander integriert. Anschließend werden sie im Rahmen eines weiteren Analysedurchlaufs überprüft und ggf. revidiert. D. h. es handelt sich bei dieser integrierten Analyse um einen dynamischen, iterativen Prozess zur Bildung von theorie- und empiriegeleitet ermittelten und rekursiv am Datenmaterial überprüften Kategorien zur optimalen Beschreibung und Erklärung eines Gegenstands bzw. zur Beantwortung der eingangs aus dem Erkenntnisinteresse abgeleiteten Forschungsfragen. Neu im Vergleich zu der von Mayring vorgeschlagenen Vorgehensweise ist die Tatsache, dass hier die Integration von deduktivem und induktivem Verfahren methodisch-methodologisch konzeptionell angelegt ist und keine Entweder-Oder-Entscheidung für die eine oder andere Vorgehensweise zu treffen ist. 


\section{Fazit und Desiderata für die künftige Methodologie- Diskussion}

Mir scheint, dass der aktuell wichtigste methodisch-methodologische (Fort-) Schritt in der QIA in der Kombination von deduktiven und induktiven Vorgehensweisen liegt. Daher möchte ich aus der Sicht der Fremdsprachenforschung dafür plädieren, diesen Schritt weiter kritisch zu diskutieren und für die Entwicklung eines integrierten Prozessmodells einer aufsteigenden, induktiven, textgeleiteten, bottom up-Analyse mit einer absteigenden deduktiven, schemageleiteten top down-Analyse zu nutzen. In diesem Zusammenhang bedarf es einer reflektierten Diskussion des Kriteriums der Gegenstandsangemessenheit, d. h. es sollte darüber nachgedacht werden, für welche Gegenstände, Erkenntnisinteressen oder Fragestellungen eine solche Vorgehensweise indiziert ist und für welche eher nicht.

Von besonderer Relevanz für die Erforschung von Lehr- und Lernprozessen erscheint mir ferner die von Rogge (in diesem Band) aufgeworfene Idee, systematisch kommunikative Validierungen durchzuführen. So wäre zu diskutieren, ob und inwiefern die theoriegeleitete Forscherperspektive und die empiriegeleitete Textperspektive zwecks Erhöhung der Validität von Ergebnissen und Interpretationen um die dialogbasierte Forschungsteilnehmer/innenperspektive erweitert werden sollte. Eine Validierung durch Forschungsteilnehmer/innen erscheint insbesondere bei der der Exploration von solchen Gegenständen sinnvoll, über die entweder nur wenig bekannt ist oder die ausschließlich mittels Erhebung, Analyse und Interpretation ,subjektiver' Daten erforschbar sind. Da die Exploration jedoch ursprünglich kein Ziel der QIA war, sehe ich auch hier reichlich methodisch-methodologischen Diskussionsbedarf.

Die Kategorienbildung - sei sie deduktiv oder induktiv - ist ein elementarer Prozess für jede geistige Tätigkeit (vgl. dazu auch Kuckartz 2012: 41) und damit auch für inhaltsanalytische Untersuchung von Texten. Wissenschaftlich nützliche Kategorien müssen zugleich genau, disjunkt und exhaustiv sein (vgl. dazu z. B. Bortz/Döring 2006: 140). Sie zu ermitteln, ist nicht trivial und bedarf eines systematischen Vorgehens. Für die künftige Analysearbeit notwendig und wünschenswert wäre hier eine vertiefte systematische methodologisch-methodische Diskussion von empirisch arbeitenden Fremdsprachendidaktikerinnen und -didaktikern hinsichtlich der Frage, wie bei der Bildung von Kategorien im Einzelnen vorzugehen ist - dem laut Beier (in diesem Band) „entscheidendste[n] und problematischste[n] Analyseschritt". 


\section{Literatur}

Bortz, Jürgen / Döring, Nicola (2006). Forschungsmethoden und Evaluation für Human- und Sozialwissenschaftler. Heidelberg: Springer, 4. überarbeitete Auflage.

Byram, Michael (1997). Teaching and assessing intercultural communicative competence. Clevedon: Multilingual Matters.

Flick, Uwe / von Kardoff, Ernst / Steinke, Ines (Hrsg.) (2000). Qualitative Forschung: Ein Handbuch. Reinbek bei Hamburg: rowohlt.

Gerbner, George / Holsti, Ole R./ Krippendorf, Klaus / Paisley, William J. / Stone, Philip J. (eds.) (1969). The analysis of communication content. New York: Wiley.

Glaser, Barney, G. / Strauss, Anselm L. (1967). The discovery of the Grounded Theory: Strategies for qualitative research. New York: Aldine de Gruyter.

Glaser, Barney G. / Strauss, Anselm L. (1998). Grounded Theory. Strategien qualitativer Forschung. Bern: Huber.

Gläser, Jochen / Laudel, Grit (2009). Experteninterviews und qualitative Inhaltsanalyse als Instrumente rekonstruierender Untersuchungen. Wiesbaden: VS Verlag für Sozialwissenschaften, 3. überarbeitete Auflage.

Groeben, Norbert / Wahl, Diethelm / Schlee, Jörg/ Scheele, Brigitte (1988). Forschungsprogramm Subjektive Theorien. Eine Einführung in die Psychologie des reflexiven Subjekts. Tübingen: Francke.

Haudeck, Helga (2005). Wie ,,pauken“ Schüler und Schülerinnen Vokabeln für den Fremdsprachenunterricht wirklich? Ein Anwendungsbeispiel der Qualitativen Inhaltsanalyse aus der Lernstrategienforschung. In: Mayring, Philipp \& Gläser-Zikuda, Michaela (Hrsg.): Die Praxis der Qualitativen Inhaltsanalyse. Weinheim \& Basel: Beltz. 84-104.

Klafki, Wolfgang (1971). Hermeneutische Verfahren in der Erziehungswissenschaft. In: Klafki, Wolfgang et al. (Hrsg.) Erziehungswissenschaft. Band 3. Frankfurt am Main: Fischer. 126-153.

Kracauer, Siegfried (1952). The challenge of qualitative content analysis. In: Public Opinion Quarterly 16: 631-642.

Krippendorf, Klaus (1969). Models of messages. In: Gerbner, George et al. (eds.): The analysis of communication content. New York: Wiley. 69-106.

Kuckartz, Udo (2012). Qualitative Inhaltsanalyse. Methoden, Praxis, Computerunterstützung. Weinheim \& Basel: Beltz Juventa.

Lamnek, Siegfried (2005). Qualitative Sozialforschung. Lehrbuch. Weinheim u. a.: Beltz.

Mayring, Philipp (1983). Qualitative Inhaltsanalyse. Grundlagen und Techniken. Beltz Verlag: Weinheim.

Mayring, Philipp (2000). Qualitative Inhaltsanalyse. In: Flick, Uwe et al. (Hrsg.): Qualitative Forschung: Ein Handbuch. Reinbek bei Hamburg: rowohlt. 468-475.

Mayring, Philipp (2010). Qualitative Inhaltsanalyse. Grundlagen und Techniken. Weinheim \& Basel: Beltz, 11. aktualisierte und überarbeitete Auflage (1. Auflage 1982). 
Miles, Matthew B. / Huberman, A. Michael (1994). Qualitative data analysis: An expanded sourcebook. London u. a.: Sage Publications Ltd.

Oevermann, Ulrich et al. (1979). Die Methodologie einer ,objektiven Hermeneutik' und ihre allgemeine forschungslogische Bedeutung in den Sozialwissenschaften. In: Soeffner, Hans-Georg (Hrsg.): Interpretative Verfahren in den Sozial- und Textwissenschaften. Stuttgart: Metzeler. 352-434.

Steigleder, Sandra (2008). Die strukturierende qualitative Inhaltsanalyse im Praxistest. Eine konstruktiv kritische Studie zur Auswertungsmethodik von Philipp Mayring. Marburg: Tectum.

Steinke, Ines (2000). Gütekriterien qualitativer Forschung. In: Flick et al. (Hrsg.): Qualitative Forschung: Ein Handbuch. Reinbek bei Hamburg: rowohlt. 319-331.

Wilden, Eva (2006). The ABC's Online. Using voice chats in a transnational foreign language teacher exchange. In Schmidt, P. Ruggiano / Finkbeiner, Claudia (Hrsg.): The ABC's of cultural understanding and communication. National and international adaptations. Greenwich, CT: Information Age Publishing. 189-212.

Wilden, Eva (2008). Selbst- und Fremdwahrnehmung in der interkulturellen Onlinekommunikation. Das Modell der ABC's of Cultural Understanding and Communication Online. Eine qualitative Studie. Frankfurt am Main: Peter Lang.

Wittgenstein, Ludwig (1953/1977). Philosophische Untersuchungen. Frankfurt am Main: Suhrkamp, 1. Auflage. 


\title{
Qualitative Inhaltsanalyse in der Bildungsforschung - Beispiele aus diversen Studien
}

\section{Michaela Gläser-Zikuda}

\begin{abstract}
The aim of this contribution is to present Qualitative Content Analysis as an analytical method for empirical studies in the context of educational research and to discuss the investigative potential of this method. First, theoretical background and main analytical techniques of Qualitative Content Analysis are explained and illustrated based on examples from different empirical studies in educational research. Furthermore, Qualitative Content Analysis is discussed with respect to the combination of qualitative and quantitative research methods in the sense of triangulation and mixed methods approaches. Finally, content analytical criteria of scientific quality and software for qualitative analysis will be addressed.
\end{abstract}

\section{Einleitung}

In der Bildungsforschung haben sich qualitative Forschungsmethoden seit einigen Jahren etabliert. Sie finden eine breite Anwendung in der erziehungswissenschaftlich, fachdidaktiktisch sowie soziologisch orientierten Bildungsforschung, und zunehmend auch aus psychologischer Perspektive. Zahlreiche bekannte und gleichsam bewährte, aber auch neue Forschungsmethoden zur Erhebung und Analyse qualitativer Daten sowie spezielle Forschungsprogramme kommen hierbei in verschiedenen Studien zur Anwendung (Breidenstein 2008, Combe et al. 1999, Helsper/Böhme 2008, Reinders et al. 2010). Zudem sind methodologische Entwicklungen innerhalb des qualitativen Paradigmas erkennbar, die sich insbesondere durch eine Ausweitung des forschungsmethodischen Repertoires kennzeichnen lassen. So gewinnen neben verschiedenen Interviewformen vor allem videogestützte Beobachtungen (z. B. v. Aufschnaiter/Welzel 2001, Seidel 2011) sowie inhaltsanalytisch basierte Auswertungen (z. B. Mayring/ Gläser-Zikuda 2008) zunehmend an Bedeutung. Darüber hinaus ist eine Zunahme an Studien zu beobachten, die sich durch eine Kombination qualitativer und quantitativer Verfahren auszeichnen (z. B. Flick 2004, Gläser-Zikuda et al. 2012, Hofmann et al. 2008).

\section{Charakteristika qualitativer Sozialforschung}

Qualitative Sozialforschung ist am geistes- und kulturwissenschaftlichen Forschungsverständnis orientiert, betrachtet das Subjekt als Konstrukteur seiner Wirklichkeit, nimmt menschliches Erleben und Verhalten in den Blick und nähert sich dem Forschungsgegenstand durch Verstehen und Interpretation 
(Flick et al. 2008, Friebertshäuser et al. 2010, Mayring 2002). Ferner ist sie um Praxisverbesserung bemüht.

Qualitative Sozialforschung ist durch Offenheit und Breite, aber auch durch Detailorientierung und Betroffenheit gekennzeichnet. Offenheit in der qualitativen Sozialforschung zeigt eine starke Abgrenzung gegenüber der quantitativen Vorgehensweise. Der Wahrnehmungsfilter soll so weit als möglich offen bleiben, damit auch nicht erwartete Informationen aufgenommen werden können. Offenheit soll gegenüber den Untersuchungspersonen, der Untersuchungssituation und den Untersuchungsmethoden herrschen. Dies führt eine Betonung der Explorationsfunktion und einen Verzicht auf eine „Hypothesenbildung ex ante“ mit sich (Lamnek 1993).

Des Weiteren macht sie die von der Forschungsfrage betroffenen Menschen zum Ausgangspunkt der Untersuchung (Subjektbezogenheit) und strebt eine möglichst genaue, vollständige und facettenreiche Darstellung des Gegenstandsbereichs an (Ganzheitlichkeit, Historizität).

Schließlich untersucht qualitative Forschung ihre Gegenstände immer möglichst in ihrem natürlichen, alltäglichen Umfeld, achtet bei der Wahl von Untersuchungsdesign und -methode auf Gegenstandsangemessenheit, setzt am einzelnen Fall sowie an praktischen Problemstellungen an und bezieht ihre Ergebnisse auf die Praxis (Friebertshäuser et al. 2010, Lamnek 1993, Mayring 2002).

Das Individuelle, Einmalige, Besondere wird beschrieben und interpretiert. Somit können wir festhalten, dass qualitative Sozialforschung insofern idiographisch ist, als sie versucht, soziale Erscheinungen in ihrem Kontext, in ihrer Komplexität und in ihrer Individualität zu erfassen, zu beschreiben und zu verstehen. (Lamnek 1993: 223)

Soziale Phänomene werden nicht kausal erklärt (wie im quantitativen Paradigma), sondern teleologisch verstanden. Dabei ist die Reflexivität des Gegenstands eine Grundannahme des qualitativ-interpretativen Paradigmas. Jede Bedeutung ist kontextgebunden, und damit verweist jede Bedeutung reflexiv auf das Ganze und wird nur durch den Rekurs auf den Kontext seiner Erscheinung verständlich. Dem entspricht die Reflexivität oder die Zirkularität der Analyse (im Sinne des ,hermeneutischen Zirkels'), der Verstehensleistung.

Die qualitative Forschung ist theorieentwickelnd oder hypothesengenerierend, d. h. die Theorie wird erst im Laufe des Forschungsprozesses gebildet. Damit ist es möglich, „den theoretischen Bezugsrahmen während des Forschungsablaufs stets zu novellieren oder sogar erst zu entwickeln" (Lamnek 1993: 225). Die qualitative Sozialforschung sieht die Hypothesenentwicklung als wesentliches Ziel an: „Hypothesen werden aus dem zu untersuchenden sozialen Feld gewonnen, d. $h$. induktiv von den Beobachtungen hin zur Theorie" (Lamnek 1993: 225).

Qualitative Sozialforschung erachtet Kommunikation zwischen Forschenden und Beforschten als konstitutives, aber auch reflexionsbedürftiges Element des 
Verstehensprozesses und geht von einem gleichberechtigten Verhältnis zwischen ForscherInnen und Beforschten aus. Da es sich einerseits um subjektive Sichtweisen sowie andererseits um Deutungen und Interpretationen handelt, wird Forschung als Interaktionsprozess aufgefasst, in dem sich Forschende und Forschungsgegenstand ändern (Forscher-Gegenstands-Interaktion) (Mayring 2002). Aber nicht nur die Forschung ist ein Prozess, sondern auch der Forschungsgegenstand, also die sozialen Phänomene unterliegen einer Prozesshaftigkeit. Soziale Phänomene sollen dokumentiert, analytisch rekonstruiert und schließlich durch das verstehende Nachvollziehen erklärt werden. Der Entstehungszusammenhang sozialer Phänomene soll also mit erfasst werden.

Vielfältige Erhebungsmethoden lassen sich in der qualitativen Sozialforschung unterscheiden. Typisch für einen fallorientierten und ganzheitlich orientierten Forschungsprozess sind z B. offene oder leitfadengestützte Einzelinterviews, Gruppendiskussion sowie teilnehmende Beobachtung (Friebertshäuser et al. 2010, Mayring 2002).

Um Daten aus Interviews, Dokumenten oder Beobachtungen auszuwerten, kann man zwischen sehr verschiedenen Ansätzen wählen: Objektive Hermeneutik, Sozialwissenschaftlich-hermeneutische Paraphrase, Psychoanalytische Textinterpretation, Biografische Methode, diskursanalytische Ansätze, Grounded Theory, Forschungsprogramm Subjektive Theorie, Typologische Analyse oder Qualitative Inhaltsanalyse (vgl. Flick et al. 2008, Friebertshäuser et al. 2010, Mey/Mruck 2010).

\section{Techniken und Analyseschritte der Qualitativen Inhaltsanalyse}

Die Qualitative Inhaltsanalyse (Mayring 2008) findet insbesondere in der Bildungsforschung sehr häufig Anwendung. Sie versteht sich als kommunikationswissenschaftliche Methode, die ursprünglich zur quantitativen Analyse von Massenmedien entwickelt wurde. Qualitative Inhaltsanalyse zielt darauf ab, manifeste und latente Sinnstrukturen interpretativ zu erschließen; sie zeichnet sich dadurch aus, dass sie das Material in den konkreten Kommunikationszusammenhang einbettet, systematisch und regelgeleitet vorgeht, die Kategorienkonstruktion und -begründung in den Mittelpunkt stellt, Verfahrensentscheidungen theoriegeleitet begründet, auch quantitative Analyseschritte als sinnvolle Ergänzung zur Deutung des Gegenstandes vorsieht und Wert auf die Überprüfung von Gütekriterien legt (Mayring 2002).

Ein regelgeleiteter Ablauf ermöglicht, dass die Analyse nachvollzogen und auf Gütekriterien hin überprüft werden kann. Das systematische Vorgehen zeigt sich außerdem darin, dass das Datenmaterial auf eine theoretische Fragestellung hin ausgewertet wird. Qualitative Inhaltsanalyse zielt darauf ab, Kategorien 
durch die Analyse des Materials zu entwickeln bzw. am Material zu überprüfen. Sowohl ein induktives als auch ein deduktives Vorgehen sind bei der Analyse möglich.

Der Ablauf der Qualitativen Inhaltsanalyse ist folgendermaßen strukturiert: Qualitative Inhaltsanalyse umfasst die Techniken der Zusammenfassung, Explikation und Strukturierung, die in sich nochmals in Analyseformen unterteilt sind. Dies heißt nicht, dass immer alle Techniken anzuwenden sind, sondern vielmehr hängt dies von der Forschungsfrage sowie einem eher induktiven oder deduktiven Vorgehen ab.

Die Qualitative Inhaltsanalyse basiert auf drei zentralen Techniken: Zusammenfassung, Explikation und Strukturierung.

\subsection{Die inhaltsanalytische Technik der Zusammenfassung}

Das Ziel der zusammenfassenden Inhaltsanalyse besteht nun darin, das Datenmaterial so zu reduzieren, dass durch Abstraktionsvorgänge ein Korpus herauskristallisiert wird, das als Abbild der Gesamtheit der Daten gelten kann. Sie folgt insofern einer induktiven Auswertungslogik. Die jeweilige Abstraktionsebene der Zusammenfassung, auf die das Material durch Einsatz der Makrooperatoren transformiert wird, muss im Vorfeld jedoch genau festgelegt werden. Sie kann dann schrittweise verallgemeinert werden, sodass die Zusammenfassung immer abstrakter wird. Grundvoraussetzung aller Analyseschritte ist selbstverständlich, dass durch die Fragestellung eine inhaltliche Eingrenzung dahingehend erfolgt, was überhaupt zusammengefasst werden soll. Das heißt, ein Selektionskriterium ist zu definieren.

Die zusammenfassende Qualitative Inhaltsanalyse eignet sich für eine Reduktion des Grundmaterials bei großen Fallzahlen oder Datenmengen, das Anfertigen kurzer Fallbeschreibungen, die induktive Kategorienbildung sowie für eine reduktive Bearbeitung von Fundstellen bei der strukturierenden Qualitativen Inhaltsanalyse.

Zunächst erfolgt die Bestimmung der Analyseeinheit, die verdeutlichen soll, welches der minimale Textteil ist, der unter eine inhaltliche Kategorie fallen kann (,Kodiereinheit'), welches der größte unter eine Kategorie fallende Textteil sein kann (,Kontexteinheit') und welche Textteile (,Auswertungseinheit') jeweils nacheinander ausgewertet werden.

Das Material wird dann zeilenweise durchgearbeitet. Alle Fundstellen werden unterstrichen bzw. markiert, wenn mit einer Auswertungssoftware wie z. B. AQUAD oder ATLAS.ti (siehe Unterkapitel 4 dieses Beitrags) gearbeitet wird und tabellarisch unter fortlaufender Nummer zusammengestellt. 
Die einzelnen Kodiereinheiten werden paraphrasiert, d. h. in eigenen Worten unter der Verwendung sprachlicher Kurzformeln und der Streichung ausschmückender Redewendungen. Keine oder wenig inhaltstragende Textbestandteile (dies hängt davon $\mathrm{ab}$, unter welcher Zielsetzung und inhaltlichen Perspektive das Material analysiert wird) werden gestrichen und die inhaltstragenden Textstellen auf eine grammatikalische Kurzform transformiert. Das angestrebte Abstraktionsniveau der ersten Reduktion wird bestimmt. Alle Paraphrasen, die unter dem Niveau liegen, werden verallgemeinert (,Makrooperator Generalisation"). Inhaltsgleiche Paraphrasen werden gestrichen, unwichtige und nichtssagende Paraphrasen werden weggelassen (,Makrooperatoren Auslassen und Selektion").

In einem zweiten Reduktionsschritt werden sich aufeinander beziehende Paraphrasen zusammengefasst (,Makrooperator Bündelung') und durch eine neue Aussage wiedergegeben (,Makrooperatoren Konstruktion und Integration'). Als Ergebnis dieser Reduktionsphasen ergibt sich eine Zusammenstellung der neuen Aussagen als Kategoriensystem, das jedoch dahingehend rücküberprüft werden muss, ob es das Ausgangsmaterial noch repräsentiert. Praktisch ist dieser Anspruch erfüllt, wenn alle ursprünglichen Paraphrasen des ersten Materialdurchganges im Kategoriensystem aufgehen.

Aus dem Kategoriensystem lassen sich in der weiteren Analyse Hauptkategorien bilden. Dies geschieht bei der zusammenfassenden Inhaltsanalyse in einem induktiven Sinne. Sofern für die Fragestellung von Bedeutung, können auch quantitative Analysen, z. B. Häufigkeitsauszählungen, vorgenommen werden. Alle Hauptkategorien, ihre Definitionen, zugehörige Ankerbeispiele und Kodierregeln können in einem Kodierleitfaden zusammengestellt werden. Tabelle 1 verdeutlicht das Vorgehen bei einer zusammenfassenden Inhaltsanalyse.

Das Beispiel stammt aus einem Forschungsprojekt, im Rahmen dessen u. a. die Lernstrategien von Schülerinnen und Schülern auf der Grundlage von Leitfadeninterviews analysiert wurden. In der Abbildung wird dargestellt, wie unter den auswertungsleitenden Aspekten der kognitiven, metakognitiven und ressourcenbezogenen Lernstrategien Fundstellen in den Interviews identifiziert und über die Auswertungsschritte der Paraphrasierung, Reduktion und Generalisierung in eine induktiv orientierte Kategorienbildung münden. Auf diese Weise gelingt eine eng an den konkreten und bewusst reflektierten Lernhandlungen orientierte Analyse von Lernstrategien. Genauere Informationen zur Auswertung sowie zum Forschungsprojekt finden sich bei Gläser-Zikuda/Lindacher/Fuß (2006) und Lindacher (2005). 
Tabelle 1: Beispiel für eine zusammenfassende Inhaltsanalyse (Lindacher 2005)

\begin{tabular}{|c|c|c|c|c|c|c|}
\hline Fall & Seite & Zeile & Nr. & Paraphrase & Generalisierung & $\begin{array}{c}\text { Kategorien der Fälle } \\
\text { A-D }\end{array}$ \\
\hline A & 66 & $\begin{array}{l}1664- \\
1665\end{array}$ & 1 & $\begin{array}{l}\text { Überblick ver- } \\
\text { schaffen über } \\
\text { Informationen im } \\
\text { Internet }\end{array}$ & $\begin{array}{l}\text { Vorbereitung der } \\
\text { Lernhandlung }\end{array}$ & $\begin{array}{l}\text { K 1: Vorbereitung und } \\
\text { Planung der } \\
\text { Lernhandlung: }\end{array}$ \\
\hline A & 66 & $\begin{array}{l}1673- \\
1675\end{array}$ & 2 & $\begin{array}{l}\text { Herausfiltern } \\
\text { wichtiger } \\
\text { Informationen }\end{array}$ & nsauswahl & \multirow{2}{*}{$\begin{array}{l}\text {-Überblick über } \\
\text { Informationen } \\
\text {-Festlegen von } \\
\text { Lernzielen } \\
\text {-Auswahl von } \\
\text { Lernstrategien } \\
\text {-Aufgabenverteilung in } \\
\text { der Gruppe }\end{array}$} \\
\hline A & 66 & $\begin{array}{l}1680- \\
1682\end{array}$ & 3 & $\begin{array}{l}\text { Lesen der Über- } \\
\text { schriften im } \\
\text { Internet, dadurch } \\
\text { Orientierung bei } \\
\text { Auswahl der } \\
\text { Inhalte }\end{array}$ & Informationsauswahl & \\
\hline A & 66 & 1684 & 4 & $\begin{array}{l}\text { Herausfiltern von } \\
\text { Informationen } \\
\text { mit Hilfe z. B. der } \\
\text { Jahreszahlen als } \\
\text { Auswahlkriterium }\end{array}$ & Informationsauswahl & \multirow{4}{*}{$\begin{array}{l}\text { K 2: Verarbeitung der } \\
\text { Informationen: } \\
\text {-Differenzierung } \\
\text { zwischen wichtigen } \\
\text { und unwichtigen } \\
\text { Inhalten (Selektion) }\end{array}$} \\
\hline A & 66 & $\begin{array}{l}1691- \\
1693\end{array}$ & 5 & $\begin{array}{l}\text { Nochmaliges } \\
\text { Aufrufen inhalt- } \\
\text { lich komplexer } \\
\text { Seiten, sobald } \\
\text { mehr Hinter- } \\
\text { grundwissen } \\
\text { vorhanden }\end{array}$ & $\begin{array}{l}\text { Assimilation neuer } \\
\text { Strukturen an } \\
\text { vorhandene } \\
\text { Strukturen }\end{array}$ & \\
\hline A & 67 & $\begin{array}{l}1698- \\
1700\end{array}$ & 6 & $\begin{array}{l}\text { Aufbauen auf } \\
\text { Vorwissen, z. B. } \\
\text { durch } \\
\text { Erzählungen der } \\
\text { Großeltern }\end{array}$ & $\begin{array}{l}\text { Assimilation neuer } \\
\text { Strukturen an } \\
\text { vorhandene } \\
\text { Strukturen }\end{array}$ & \\
\hline A & 68 & $\begin{array}{l}1763- \\
1764\end{array}$ & 7 & $\begin{array}{l}\text { Ziel vorge- } \\
\text { nommen für } \\
\text { einen Tag }\end{array}$ & $\begin{array}{l}\text { Formulierung von } \\
\text { Lernzielen }\end{array}$ & \\
\hline
\end{tabular}

Qualitative Inhaltsanalyse ermöglicht es zudem, Quantifizierungen vorzunehmen, wenn es für die Forschungsfrage relevant erscheint. Im Fall der zusammenfassenden Inhaltsanalyse lassen sich Häufigkeitsangaben für die induktiv gebildeten Kategorien bzw. Hauptkategorien angeben, um z. B. eine Einschätzung besonders häufig betonter Aspekte eines Gesprächsthemas vornehmen zu können. In einem Forschungsprojekt aus dem Bereich Englischdidaktik verfolgte Haudeck (2008) die Frage, wie Schülerinnen und Schüler Vokabeln lernen. 
Tabelle 2: Beispiel für die Ergebnisdarstellung der zusammenfassenden Inhaltsanalyse mit Häufigkeitsangaben (Haudeck 2008, vgl. Mayring/Gläser-Zikuda 2008: 91)

\begin{tabular}{|l|l|c|c|}
\hline \multicolumn{1}{|c|}{ Die Kategorien der am häufigsten genannten Lernaktivitäten/Lerntechniken (n=142) } \\
\hline \multicolumn{1}{|c|}{ Kategorien } & Nennungen & \% \\
\hline K9 & $\begin{array}{l}\text { zudecken, abdecken, zuheben, Seiten abwechselnd } \\
\text { verdecken, dt. bzw. engl. Bedeutung verdecken }\end{array}$ & 69 & 48,6 \\
\hline K3 & $\begin{array}{l}\text { 1-2x, 2-3x, 4x, öfters, paarmal, mehrmals durchlesen } \\
\text { wissen }\end{array}$ & 47 & 33,1 \\
\hline K5 & durchlesen (anschauen, durchgucken) & 42 & 29,6 \\
\hline K16 & sich (oft) abfragen lassen (mündlich) & 40 & 28,2 \\
\hline K8 & Vokabeln (auf-)schreiben, schriftlich lernen & 19 & 14,1 \\
\hline
\end{tabular}

Hierzu ließ sie die Schülerinnen und Schüler über mehrere Wochen ein Lerntagebuch zu schulischen und insbesondere häuslichen Lernsituationen in schriftlicher Form oder als Audioversion führen. Die Notizen bzw. transkribierten Aufnahmen wurden induktiv mittels einer zusammenfassenden Inhaltsanalyse ausgewertet. Um einen Eindruck von der Frequenz der von den Lernenden bewusst genutzten Lernstrategien zum Vokabellernen zu erhalten, zählte die Forscherin die Anzahl der ermittelten Kategorien über alle Lerntagebücher der Schülerinnen und Schüler hinweg aus (vgl. Tabelle 2). Auf diese Weise lassen sich hinsichtlich der Forschungsfrage auch Untergruppenvergleiche vornehmen, so z. B. die Gegenüberstellung von Angaben von Mädchen und Jungen oder von verschiedenen Schülergruppen (Schülerinnen und Schüler mit unterschiedlichen Lernleistungen oder mit bzw. ohne Migrationshintergrund etc.).

\subsection{Die inhaltsanalytische Technik der Explikation}

Die explizierende Inhaltsanalyse kann sich an die oben erläuterten reduktiven Prozeduren anschließen, wenn fragliche Textteile übrig bleiben, deren Verständnis durch die Analyse zusätzlichen Materials erhellt wird. Sie verhält sich zum Ziel der zusammenfassenden Inhaltsanalyse insofern genau umgekehrt, als sie nicht die Reduktion, sondern die Erweiterung der Textstellen anstrebt, um sie zu erklären. Das Wesentliche dieser Technik besteht darin, aufgrund definierter ,Quellen' zu einer plausiblen Aufschlüsselung zunächst unklarer Aussagen zu gelangen. Die Grundlage jeder Explikation ist die lexikalisch-grammatikalische Definition. Meist findet man in Interviews jedoch Aussagen, die von diesen verbindlichen Standards abweichen, denn jedes Individuum gibt seiner Sprache eine spezifisch eigene Bedeutung. Es gilt dann, auf den Kontext der Äußerungen zurückzugreifen. Je nachdem, ob dabei nur andere Textstellen aus demselben Material oder auch über den eigentlichen Text hinausgehende Informationen zugelassen werden, wird zwischen der engen und der weiten Kontextanalyse unterschieden. 


\subsection{Die inhaltsanalytische Technik der Strukturierung}

Einer eher deduktiven Logik folgend verfährt die strukturierende Inhaltsanalyse. Das Kernstück jeder strukturierenden Inhaltsanalyse ist dabei die Zusammenstellung eines Kategoriensystems mit Definitionen, Ankerbeispielen, Kodierregeln und Fundstellenbezeichnungen. Grundsätzlich müssen die Strukturierungsdimensionen aus der Fragestellung abgeleitet und theoretisch begründet werden. Eventuell lassen sie sich auch weiter in einzelne Ausprägungen differenzieren. Wann ein Materialbestandteil unter eine Kategorie fällt, bedarf präziser - wiederum theoriegeleiteter - Kategoriendefinitionen. Zusätzlich werden als Beispiele Textstellen angeführt, die eine Kategorie bestmöglich repräsentieren, sogenannte ,Ankerbeispiele‘. Ein Regelwerk zur Kodierung stellt sicher, dass Materialzuordnungen eindeutig erfolgen können und Abgrenzungsprobleme zwischen den Kategorien vermieden werden.

Der idealtypische Ablauf einer strukturierenden Qualitativen Inhaltsanalyse gestaltet sich folgendermaßen (vgl. Mayring 2008):

1. Bestimmung des Ausgangsmaterials,

2. Fragestellung der Analyse,

3. Bestimmung der Analyseeinheit,

4. Zusammenstellung des Kategoriensystems,

5. Materialdurchlauf,

6. Fundstellenbezeichnung,

7. Bearbeitung und Extraktion der Fundstellen,

8. Ergebnisaufbereitung.

Folgende Varianten der strukturierenden Inhaltsanalyse bieten sich an:

(1) Ein Text wird nach syntaktischen, thematischen, semantischen oder dialogischen Kriterien analysiert (,formale Strukturierung").

In einem Forschungsprojekt zu Bildungsstandards für das Abitur im Fach Deutsch wurden Interpretationsleistungen in Abiturprüfungsarbeiten inhaltsanalytisch ausgewertet. Es wurde davon ausgegangen, dass jede schriftliche Interpretationsäußerung entweder beschreibend, deutend oder wertend und keine Äußerung das eine und das andere zugleich ist. Um zu veranschaulichen, wo in den schriftlichen Abiturarbeiten beschrieben, gedeutet und wo gewertet wurde, führte Steinmetz (2011) eine formale Strukturierung von Prüfungsarbeiten auf der Grundlage einer Stichprobe von $\mathrm{N}=50$ Abiturarbeiten eines Jahrgangs durch (vgl. Abbildung 1). Vorab formulierte er deduktiv-theoriegeleitet drei Kategorien, die zur Analyse herangezogen wurden. 


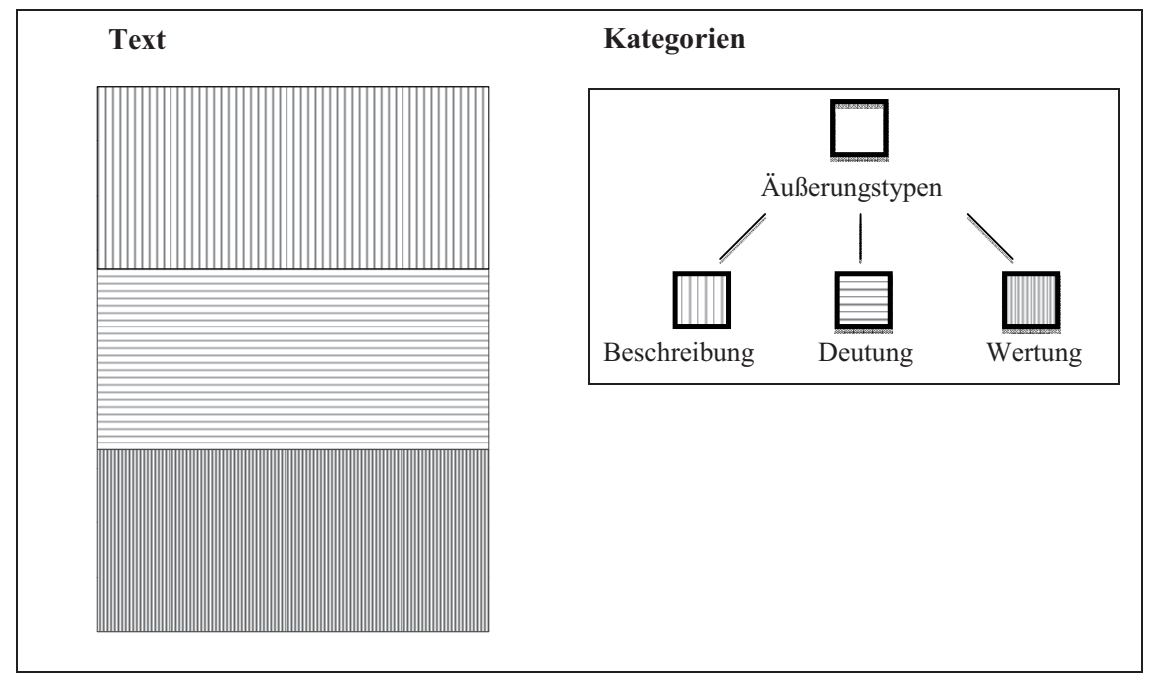

Abbildung 1: Formale Strukturierung eines Textes („Textportrait“; Steinmetz 2011: 77)

Die Unterscheidung der Äußerungen eines Textes nach ihrer Funktion lassen sich relativ abstrakt mittels der Kategorien ,Beschreibung', ,Deutung ' und ,Wertung' realisieren. Die beschreibenden Äußerungen wurden weiter in formund inhaltsbeschreibende Äußerungen ausdifferenziert. Formbeschreibende Äußerungen wiederum ließen sich nach narratologischen, stilistischen und grammatischen Aspekten unterscheiden. Auf diese Weise ließen sich verschiedene Interpretationsmuster ermitteln.

(2) Die typisierende Strukturierung befasst sich mit markanten Ausprägungen auf einer Typisierungsdimension, z. B. mit häufig auftretenden oder beobachteten bzw. theoretisch interessanten Aspekten. So lassen sich auf der Grundlage von Schülerinterviews und lernprozessbezogenen Beobachtungen beispielsweise spezifische Lerntypen herausarbeiten (Gläser-Zikuda 2001). In einem ersten Schritt wurden aus Leitfadeninterviews und Lerntagebüchern induktiv zusammenfassend für insgesamt 24 Schülerinnen und Schüler der Sekundarstufe I Lernstrategien und Emotionen im Kontext von Schule und Unterricht analysiert. Für jeden Einzelfall wurde dann in einem zweiten Auswertungsschritt eine Gesamtcharakterisierung vorgenommen. Schließlich wurde das Material nach besonders häufigen bzw. seltenen Fällen gesichtet. Der im Datenmaterial der genannten Studie am häufigsten ermittelte Lerntyp ließ sich als sogenannter ,Notenorientierter Lust-/Unlust-Typus' charakterisieren.

Bei dieser Schülergruppe fiel auf, dass sie im Vergleich zu einer weiteren, nicht so zahlreich vertretenen Gruppe (sogenannter ,Lernfreude-Typus') insgesamt weniger positiv bzgl. schulischer Belange eingestellt war. Positive, aber 
auch negative Gefühle wurden in den Interviews und Lerntagebüchern überwiegend mit Situationen verknüpft, die durch Leistungserfolg oder -versagen gekennzeichnet waren. Auf den Punkt gebracht verdeutlichen die Aussagen dieser Schülergruppe Folgendes: „Schule gefällt mir, wenn ich gute Noten bekomme." Das Darstellungsmuster für diesen Typus stellt sich folgendermaßen dar:

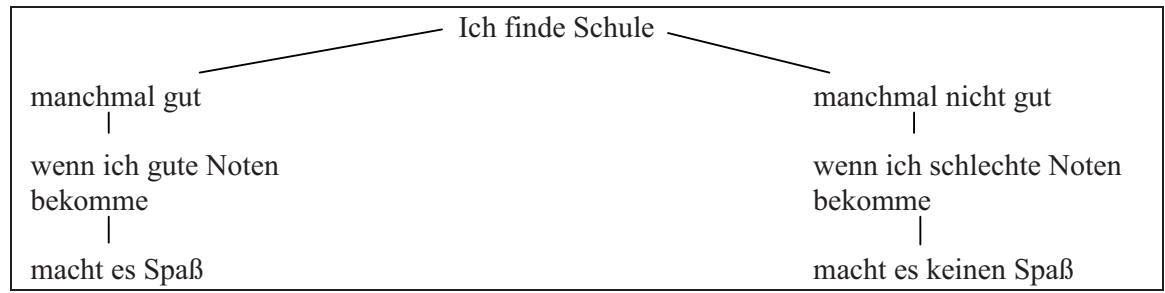

Abbildung 2: Typisierende Strukturierung (Gläser-Zikuda 2001: 245)

Folgende Interview-Ausschnitte beschreiben das im obigen Darstellungsmuster Aufgezeigte.

Weil ich so gut bin, macht es mir Spaß. Sonst würde es mir wahrscheinlich weniger Spaß machen. (Fall 113)

Die Fächer machen halt Spaß und da bin ich halt gut. (...) Rechtschreiben ist nicht so toll, da bin ich halt nicht gut. Da mache ich viele Leichtsinnsfehler. (Fall 540)

Diese Schülergruppe verbindet Freude am Lernen und an der Schule mit guten Noten. Schlechte Noten bewirken dann entsprechend weniger Spaß bzw. erzeugen Langweile. Angst wird von dieser Schülergruppe nicht oder nur sehr selten genannt. Diese Schülerinnen und Schüler sind insgesamt eher leistungsstark und setzen überwiegend elaborative und zum Teil auch metakognitive Strategien ein. Sie wenden aber auch oberflächliche Strategien an, und zwar vor allem dann, wenn sie nicht besonders motiviert sind, sich auf eine Klassenarbeit vorzubereiten. Mittels typisierender Strukturierung ließen sich in der vorliegenden Studie mehrere Typen ermitteln und darüber hinaus wichtige Informationen und Annahmen für weiterführende Studien generieren.

(3) Der skalierenden Strukturierung schließlich geht es darum, das Material auf einer Skala einzuschätzen; in der Regel wird das Ordinalskalenniveau zu Grunde gelegt. So können beispielsweise die Kompetenzen von Lehrpersonen aus Sicht von Schülern aus Interviewdaten hinsichtlich ihrer Ausprägung interpretiert und eingeschätzt werden. Damit eröffnen sich auch Möglichkeiten der Verknüpfung mit quantitativen Daten (vgl. Beispiel in Tabelle 3; siehe auch Tabelle 5). 
Tabelle 3: Beispiel für eine skalierende Strukturierung (Gläser-Zikuda/Fuß 2008: 122)

\begin{tabular}{|c|c|c|}
\hline $\mathrm{Ni}$ & Definition & Ankerbeispiele \\
\hline $\begin{array}{l}\text { Hohes } \\
\text { Niveau } \\
\text { an } \\
\text { Klarheit } \\
\text { (C1) }\end{array}$ & $\begin{array}{l}\text { Der Lehrer (1) ist stets akustisch } \\
\text { und visuell klar strukturiert und } \\
\text { verständlich, (2) setzt mehrere } \\
\text { Male advance organizers ein, (3) } \\
\text { erläutert mehrere Male die } \\
\text { Lernziele und (4) gibt mehrere } \\
\text { Male inhaltliche Zusammen- } \\
\text { fassungen. }\end{array}$ & $\begin{array}{l}\text { (1) „Wir können immer gut sehen, was er an } \\
\text { die Tafel schreibt und man versteht ihn auch } \\
\text { gut.” (2) "Der Lehrer kommt rein und sagt } \\
\text { uns immer zu Beginn einer Stunde, was wir } \\
\text { dann machen werden.” (3) „Er sagt uns auch } \\
\text { oft, warum wir das und das lernen sollen.” (4) } \\
\text { „Nach den Experimenten oder so sagt er uns } \\
\text { oft, das habt ihr jetzt gelernt.” }\end{array}$ \\
\hline $\begin{array}{l}\text { Mittleres } \\
\text { Niveau } \\
\text { an } \\
\text { Klarheit } \\
\text { (C2) }\end{array}$ & $\begin{array}{l}\text { Ein mittleres Niveau ist angezeigt, } \\
\text { wenn die oben definierten } \\
\text { Merkmale alle oder zum Teil nur } \\
\text { vereinzelt auftreten. }\end{array}$ & $\begin{array}{l}\text { (1) „Manchmal erklärt er zu viel und dann } \\
\text { wissen wir nicht, wie wir die Aufgabe nun } \\
\text { bearbeiten sollen.“ (2) „Ich weiß nicht, was } \\
\text { ich auf seine Frage antworten soll. Die sind } \\
\text { zu komplex.“ (3) „Manchmal gibt's einen } \\
\text { kleinen Tipp, wie wir eine Aufgabe angehen } \\
\text { sollen.“ }\end{array}$ \\
\hline \begin{tabular}{l|} 
Niedriges \\
Niveau \\
an \\
Klarheit \\
(C3)
\end{tabular} & $\begin{array}{l}\text { Der Lehrer (1) ist akustisch und } \\
\text { visuell nicht klar strukturiert und } \\
\text { verständlich, (2) setzt nie advance } \\
\text { organizers ein, (3) erläutert nie die } \\
\text { Lernziele und (4) gibt nie } \\
\text { inhaltliche Zusammenfassungen. }\end{array}$ & $\begin{array}{l}\text { (1) „Seine Erklärungen sind zu schwierig und } \\
\text { er spricht über tausend andere Dinge.” (2), „In } \\
\text { seinem Unterricht weiß ich nie, was auf mich } \\
\text { zukommt.” (3),WWir wissen nie, worauf es } \\
\text { ankommt. Er sagt es uns einfach nicht.” (4) } \\
\text { „Es geht immer weiter und er erklärt nicht, so } \\
\text { das ist mal das, was wir jetzt gelernt haben.“" }\end{array}$ \\
\hline \multicolumn{3}{|c|}{$\begin{array}{l}\text { Kodierregeln: (1) Zur Zuordnung auf Niveau C1 müssen alle vier Aspekte auf einem hohen } \\
\text { Niveau ausgeprägt sein. Ansonsten erfolgt die Zuordnung zum mittleren Niveau. (2) C2 soll } \\
\text { kodiert werden, wenn nur einige der Aspekte einzustufen sind bzw. nicht alle auf einem } \\
\text { hohen Niveau angesiedelt sind. (3) C3 wird kodiert, wenn alle vier Aspekte eindeutig auf } \\
\text { niedrigem Niveau ausgeprägt sind. }\end{array}$} \\
\hline
\end{tabular}

Im qualitativen Teil der Studie zum Zusammenhang zwischen Lehrerkompetenzen und Schüleremotionen (Gläser-Zikuda/Fuß 2008) wurden halbstrukturierte Interviews mit Schülerinnen und Schülern zur Wahrnehmung ihrer Physiklehrperson geführt. Die transkribierten Daten wurden mit Hilfe einer skalierenden Strukturierung ausgewertet. Eine Expertin und ein Experte aus dem pädagogischen Bereich schätzten die Schüleräußerungen in den Interviews in Orientierung an den theoretisch begründeten Lehrerkompetenzen mittels skalierender Strukturierung ein. Theoriegeleitet wurden klare Kriterien definiert und Kodierregeln vorgegeben. Mit Hilfe sogenannter Ankerbeispiele aus dem Originalmaterial konnte die Zuordnung zu den einzelnen rangskalierten Einstufungen objektiv vorgenommen werden. So ließen sich für jede Lehrperson aus der Sicht von je acht Schülerinnen und Schülern Einschätzungen vornehmen, die in einem zweiten Schritt mit quantitativen Daten aus Emotions- 
fragebögen korreliert werden konnten. Des Weiteren erlaubte dieses Vorgehen eine gegenseitige Validierung der qualitativen und quantitativen Daten (siehe Kapitel 3.5 und Tabelle 5 in diesem Beitrag).

\section{Gütekriterien Qualitativer Inhaltsanalyse}

Um die Qualität, also die Güte des Forschungsprozesses einschätzen zu können und damit das wissenschaftliche Vorgehen abzusichern, sind gewisse Maßstäbe notwendig. Da klassische Kriterien der quantitativen Sozialforschung (Objektivität, Reliabilität und Validität) für qualitative Zielsetzungen nicht geeignet sind, wurden spezifisch qualitative Gütekriterien entwickelt, die in der Qualitativen Inhaltsanalyse berücksichtigt werden.

Tabelle 4: Regelgeleitetes Vorgehen bei der strukturierenden Inhaltsanalyse (Gläser-Zikuda 2001: 128)

\begin{tabular}{|c|c|c|c|}
\hline Variable & Definition & Ankerbeispiele & Kodierregeln \\
\hline $\begin{array}{l}\text { starkes } \\
\text { Interesse }\end{array}$ & $\begin{array}{l}\text { starke Beziehung } \\
\text { zwischen Lerner und } \\
\text { Lerngegenstand } \\
\text { schließt mit ein: } \\
\text { - } \quad \text { hohe positive } \\
\quad \text { Bewertung (Wert } \\
\quad \text { - Komponente) } \\
\text { - hohe Neugier } \\
\quad \text { (kognitive } \\
\text { Komponente) }\end{array}$ & $\begin{array}{l}\text { "Ich möchte mehr über } \\
\text { Elektrizität erfahren.” } \\
\text { „Es ist wichtig für den } \\
\text { Beruf.” } \\
\text { „Ich war überrascht } \\
\text { über den Magnetismus } \\
\text { der Tafel.” } \\
\text { „Du brauchst } \\
\text { Physikwissen im } \\
\text { Alltagsleben.” }\end{array}$ & $\begin{array}{l}\text { Gesamteindruck der Inter- } \\
\text { viewdaten und zusätzlich mehr } \\
\text { als } 80 \% \text { der Eintragungen im } \\
\text { Tagebuch zeigen hohes } \\
\text { Interesse. }\end{array}$ \\
\hline $\begin{array}{l}\text { etwas } \\
\text { Interesse }\end{array}$ & $\begin{array}{l}\text { einige Aspekte des } \\
\text { Lerngegenstandes } \\
\text { bewirken Interesse, } \\
\text { positive und negative } \\
\text { Bewertung oder nur } \\
\text { eine schwach } \\
\text { positive Bewertung } \\
\text { des Gegenstandes }\end{array}$ & $\begin{array}{l}\text { "Ich mag Experimente, } \\
\text { sie sind interessant.” } \\
\text { „Wir können bei den } \\
\text { Experimenten mit dem } \\
\text { Gerät arbeiten.” } \\
\text { „Neue Themen sind } \\
\text { interessant.” }\end{array}$ & $\begin{array}{l}\text { Gesamteindruck aus den } \\
\text { Interviews und weniger als } 80 \\
\% \text { der Tagebucheintragungen } \\
\text { deuten auf etwas Interesse hin. }\end{array}$ \\
\hline $\begin{array}{l}\text { kein } \\
\text { Interesse }\end{array}$ & $\begin{array}{l}\text { keine Beziehung zum } \\
\text { Lerngegenstand, } \\
\text { negative Bewertung } \\
\text { des Gegenstandes als } \\
\text { Ganzes, kein Inte- } \\
\text { resse am Lerngegen- } \\
\text { stand erkennbar }\end{array}$ & $\begin{array}{l}\text { „Physik ist überhaupt } \\
\text { nicht interessant, weil } \\
\text { wir immer wieder über } \\
\text { dasselbe Experiment } \\
\text { reden.“ „Wozu brauche } \\
\text { ich Physik?“" }\end{array}$ & $\begin{array}{l}\text { insgesamt negativer Eindruck } \\
\text { und maximal } 20 \% \text { der } \\
\text { Tagebucheintragungen }\end{array}$ \\
\hline
\end{tabular}

Spezifisch qualitative Gütekriterien können den Ansprüchen qualitativer Forschung eher gerecht werden, und sie gewährleisten die Grundprinzipien der 
Wissenschaftlichkeit. Dies sind: Verfahrensdokumentation, Regelgeleitetheit, Intercoder-Reliabilität, Kommunikative Validierung und Kombination qualitativer und quantitativer Analyseschritte.

\subsection{Verfahrensdokumentation}

Da nicht wie in quantitativer Sozialforschung geprüfte, standardisierte Erhebungsverfahren eingesetzt, sondern angepasst an den Forschungsgegenstand meist neu entwickelt werden, ist es für qualitative Sozialforschung besonders wichtig, das Vorgehen im Forschungsprozess genau festzuhalten. Je genauer dies geschieht, desto besser wird der gesamte Prozess für Dritte nachvollziehbar. Dazu gehören die Explikation des Vorverständnisses des Forschenden, die Überlegungen, die zur Auswahl der Forschungsteilnehmerinnen und -teilnehmer führten, die Entwicklung und Zusammenstellung der Erhebungsmethoden, die Durchführung und Aufbereitung der Erhebung sowie die Wahl und das Vorgehen bei der Auswertung der Daten.

\subsection{Regelgeleitetheit}

Qualitative Forschung beabsichtigt, offen gegenüber dem Forschungsgegenstand zu sein, und schließt die Änderung von Verfahrensschritten und methodischen Vorgehens mit ein (vgl. Beispiel in Tabelle 4). Allerdings darf dies nicht in einer völlig unsystematischen und nicht mehr nachvollziehbaren Art und Weise geschehen. Auch qualitative Forschung muss sich an bestimmte Verfahrensregeln halten, so z. B. ein schrittweises, sequentielles Vorgehen, die Festlegung von sinnvollen Materialeinheiten innerhalb der Daten und die Formulierung von Auswertungsregeln. Hierdurch gelingt es, ein systematisches und dadurch nachvollziehbares Vorgehen im Forschungsprozess zu gewährleisten, ohne Gefahr zu laufen, durch zu enge Vorgaben die prinzipielle Offenheit des Forschungsansatzes einzuschränken.

\subsection{Intercoder-Reliabilität}

Um Aussagen über die Zuverlässigkeit der Interpretationen innerhalb der in einer Studie angewandten Verfahren $\mathrm{zu}$ treffen, lässt sich die sogenannte Intercoder-Reliabilität ermitteln, ein $\mathrm{Maß}$ zur Prüfung der Übereinstimmung zwischen verschiedenen Auswerterinnen und Auswertern (vgl. Mayring 2008). Insbesondere spielen Stabilität (die nochmalige Anwendung des Analyseinstruments), Reproduzierbarkeit (der Grad, zu dem die Analyse bei anderen Analytikern zu denselben Ergebnissen führt) sowie Exaktheit (der Grad, zu dem das Vorgehen einem bestimmten funktionellen Standard entspricht) eine wichtige Rolle. Handelt es sich um zwei Beobachter, die gleichzeitig mehrere Beob- 
achtungsobjekte (also Fälle bzw. Probanden) kategorial einschätzen, so lässt sich die Interrater-Reliabilität mittels Cohens Kappa ermitteln. Dabei wird das Ausmaß an Übereinstimmung durch Einbezug und Vergleich zu dem durch ,zufälliges Einschätzen“ typischerweise erreichbaren Ausmaß an Übereinstimmung. Es wird davon ausgegangen, dass die einzelnen Einschätzungen eines Raters vollkommen unabhängig voneinander getroffen werden. Kappa nimmt Variablen auf Nominalskalenniveau an und kann Werte zwischen +1 und 0 annehmen (Wirtz/Caspar 2002).

Prinzipiell empfehlenswert ist es, die Intercoder-Reliabilität nach Krippendorffs Alpha (2004) zu ermitteln. Krippendorffs Alpha (für Nominaldaten) vergleicht die Kodierungen auf Übereinstimmung mit Berücksichtigung der Reihenfolge und Korrektur nach den zufällig zu erwartenden Übereinstimmungen. Reliabilitätstests haben prinzipiell einen schätzenden Charakter, d. h. demzufolge sind Reliabilitätswerte also Schätzwerte für die ,wahre', aber unbekannte Reliabilität. Die Präzision von Schätzwerten hängt u. a. von der Stichprobengröße bzw. Datenmenge ab. Die Intercoder-Reliabilität kann demzufolge mit diesem Koeffizienten berechnet werden, der nicht nur die Quote der übereinstimmenden Kodierungen verschiedener Auswerter berücksichtigt, sondern das Ergebnis um die Zahl der zufällig zu erwartenden Übereinstimmungen bereinigt (vgl. Abbildung 3).

\subsection{Kommunikative Validierung}

Die Gültigkeit der Ergebnisse sowie der Interpretation lassen sich im Rahmen qualitativer Forschung überprüfen, indem man sie den Forschungsteilnehmerinnen und -teilnehmern, den Beforschten, nochmals vorlegt und mit ihnen diskutiert. Wenn sich die Beforschten mit ihren Ansichten, Meinungen oder Haltungen in der vom Forschenden dargelegten Interpretation der Ergebnisse wiederfinden, kann dies als wichtiger Beitrag zur Absicherung der Forschungsergebnisse betrachtet werden. Neben dem Konsens zwischen Forschenden und Beforschten (kommunikative Validierung) sollte ein Konsens zwischen Interpretierenden in einer Auswertungsgruppe (konsensuelle Validierung) sowie ein Konsens zwischen Forschenden und außenstehenden Personen (argumentative Validierung) in Betracht gezogen werden. Darüber hinaus ist zu bedenken, dass sich die Interpretation selbstverständlich auch an bereits bestehenden theoretischen Modellen und Ansätzen orientieren bzw. zur Entwicklung neuer Theorien herangezogen werden sollte. Auch ist der Vergleich mit ähnlichen Studien im qualitativen und quantitativen Bereich sinnvoll, um die aktuelle Studie einordnen zu können. 
Reliabilitätsprüfung für die induktive Inhaltsanalyse: In einer ersten Reliabilitätsprüfung (,Formative Reliabilitätsprüfung“) wurden vier zufällig ausgewählte Lern- und Emotionstagebücher der Studie herangezogen und durch zwei Auswerter analysiert. Es fanden sich insgesamt 120 Einträge, die kodiert wurden. Dabei wurden 95 Übereinstimmungen und 25 Nicht-Übereinstimmungen festgestellt. Daraus ergibt sich eine Übereinstimmung von $79 \%$. Die zufällig zu erwartende prozentuale Übereinstimmung wird bei vier Kodiermöglichkeiten auf $25 \%$ festgelegt.

$\mathrm{K}=\frac{79 \%-25 \%}{1-25 \%}=.72$

Der Koeffizient von .72 kann als akzeptabel beurteilt werden. Allerdings zeigte sich bei näherer Betrachtung der Nicht-Übereinstimmungen, dass es sich bei 22 Kodierungen um Folgefehler einer einzigen Kategorie handelte, die sich auf eine als zunächst oberflächliche Lernaktivität bezog. Nach theoretischen Überlegungen wurde diese Kategorie der Hauptkategorie „Elaboration“ zugeordnet. Nach dieser Korrektur des Kategoriensystems wurde nochmals eine Reliabilitätsprüfung vorgenommen (vgl. „Summative Intercoderreliabilität"). Nun ergaben sich bei den 120 Einträgen derselben vier Tagebücher nur 3 Nicht-Übereinstimmungen. Somit liegt die Übereinstimmung zwischen beiden Kodierern bei $98 \%$.

$\mathrm{K}=\frac{98 \%-25 \%}{1-25 \%}=.97$

Die Intercoder-Reliabilität ist mit .97 als sehr gut zu bezeichnen. Es ist davon auszugehen, dass das in dieser Studie angewandte Verfahren sowie der Ablauf der induktiven Inhaltsanalyse als hoch reliabel angesehen werden kann.

Abbildung 3: Beispiel für die Berechnung der Intercoder-Reliabilität (Gläser-Zikuda 2001: 218f.

\subsection{Kombination qualitativer und quantitativer Analyseschritte}

Eine Kombination qualitativer und quantitativer Analyseschritte, d. h. ein multimethodisches bzw. triangulatorisches Vorgehen ist einem einseitig methodisch ausgerichteten Ansatz vorzuziehen (Flick 2004). Im Sinne eines gemeinsamen Forschungsmodells handelt es sich bei qualitativen und quantitativen Methoden um einzelne Forschungsschritte, die miteinander verbunden werden können (Mayring 2001). Gerade eine Kombination erweitert den Erkenntnishorizont, Schwächen der jeweiligen Einzelmethode können kompensiert werden. Im angloamerikanischen Raum wird dieses Thema unter dem Schlagwort mixed methodology bzw. mixed-model designs diskutiert (Tashakkori/Teddlie 1998, 2003). Mittlerweile wird die Kombination qualitativer und quantitativer Forschungsmethoden auch in der Bildungsforschung intensiv diskutiert (z. B. Gläser-Zikuda et al. 2012).

Neben der Art der Untersuchung (explorativ oder konfirmatorisch; qualitativ und quantitativ) sowie der Art der Datenerfassung und -sammlung (qualitativ und quantitativ) ist die Art der Analyse (qualitativ und quantitativ) gerade für die Qualitative Inhaltsanalyse eine wichtige Frage. Durch die Techniken der Zusammenfassung und Strukturierung ergeben sich vielfältige Möglichkeiten. 
So können beispielsweise Häufigkeitsanalysen auf der Grundlage der zusammenfassenden Inhaltsanalyse in Form von Kategorien erfolgen.

Im Fall der strukturierenden Inhaltsanalyse (speziell der skalierenden Strukturierung) ist eine direkte Verknüpfung mit quantitativ erhobenen Daten (z. B. aus einem Fragebogen) möglich. Der gemeinsame Einsatz von qualitativen und quantitativen Methoden bedeutet nicht nur, ein und dasselbe Phänomen von unterschiedlichen Perspektiven her zu betrachten, sondern zugleich ein besseres Verstehen und Erklären, indem man tiefer in die Materie eindringen und neue Dimensionen entdecken kann.

In der bereits erwähnten Studie von Gläser-Zikuda/Fuß (2008) wurden Lehrerkompetenzen sowohl per Schülerinterview als auch mittels standardisiertem Fragebogen aus jeweils zwei Schulklassen, die von derselben Lehrperson unterrichtet wurden, erfasst. Es interessierte zum einen, wie die verschiedenen Schulklassenmittelwerte sich zu einer Auswahl von je vier Schülereinschätzungen pro Klasse verhielten. Zum anderen sollte geklärt werden, ob die auf der Grundlage der Schülerinterviews mittels skalierender Strukturierung ausgewerteten Experteninterpretationen (siehe Tabelle 3, Kapitel 2.1.3 dieses Beitrags) mit den quantitativen Daten der Schulklassenmittelwerte sowie den Teilstichproben in ihrer Tendenz vergleichbar sein würden. Zunächst ließ sich durch den rein quantitativen Vergleich der beiden Schulklassenkennwerte der drei Lehrpersonen mit den Kennwerten der drei Interviewstichproben zeigen, dass die Teilstichproben der Interviewstudie weitgehend als repräsentativ für die Beschreibung und Einschätzung der Lehrerkompetenzen angesehen werden können. Durch diesen ersten Vergleich konnten in dieser Studie grobe Stichprobenfehler, die bei der Ziehung kleiner Stichproben auftreten können, ausgeschlossen werden.

In einem zweiten Schritt erfolgte eine inhaltliche Validierung der quantitativen Daten auf der Grundlage der Ergebnisse aus den Interviews. Für die Lehrerkompetenzen ,Klarheit der Instruktion', ,Motivierungsqualität' und ,Lehrerfürsorglichkeit‘ ließen sich kongruente Ergebnisse zwischen den auf den Schülerinterviews basierenden Expertenratings und den quantitativen Daten der beiden Schulklassen einer Lehrperson ermitteln. In Tabelle 5 sind für die drei Lehrpersonen Nr. 4, Nr. 6 und Nr. 8 die Ergebnisse der Kreuzvalidierung von quantitativen und qualitativen Daten wiedergegeben. Für alle Variablen der Lehrerkompetenzen sind die Mittelwerte und Standardabweichungen für jeweils beide Klassen der Lehrpersonen (T), für die Teilstichprobe (Q; besteht aus bis zu 8 Schülerinnen und Schülern aus den beiden Klassen) und der Rang des Expertenratings (E/I) als Teil der qualitativen Analyse angegeben. Vergleicht man qualitative (Expertenratings aus den Schülerinterviews) und quantitative Daten (aus den Fragebögen) miteinander, so sind die Resultate für die Kompetenzbereiche Klarheit der Instruktion, Motivierungsqualität und Fürsorg- 
lichkeit der Lehrkraft konsistent. Es liegen lediglich für den Kompetenzbereich ,Diagnostik im Leistungsbereich“ im Fall 4 Differenzen vor.

Tabelle 5: Beispiel für die Kombination qualitativer und quantitativer Analyseschritte auf der Grundlage der skalierenden Strukturierung (Gläser-Zikuda/Fuß 2008: 129)

\begin{tabular}{|c|c|c|c|c|c|c|c|c|c|}
\hline \multirow[b]{2}{*}{ Kompetenzen } & \multicolumn{3}{|c|}{ Lehrperson 4} & \multicolumn{3}{|c|}{ Lehrperson 6 (O) } & \multicolumn{3}{|c|}{ Lehrperson 8 (X) } \\
\hline & $\begin{array}{c}M(S D) \\
T \\
n=50\end{array}$ & $\begin{array}{c}M(S D) \\
Q \\
n=7\end{array}$ & $\begin{array}{c}\text { Level } \\
\mathrm{E} / \mathrm{I} \\
\mathrm{n}=8\end{array}$ & $\begin{array}{c}M(S D) \\
T \\
n=47\end{array}$ & $\begin{array}{c}M(S D) \\
Q \\
n=8\end{array}$ & $\begin{array}{c}\text { Level } \\
E / / \\
n=8\end{array}$ & $\begin{array}{c}M(S D) \\
T \\
n=55\end{array}$ & $\begin{array}{c}M(S D) \\
Q \\
n=7\end{array}$ & $\begin{array}{l}\text { Level } \\
\mathrm{E} / \mathrm{I} \\
\mathrm{n}=8\end{array}$ \\
\hline Klarhe it & $\begin{array}{l}1.63 \\
(.61)\end{array}$ & $\begin{array}{l}1.68 \\
(.66)\end{array}$ & 1 & $\begin{array}{l}4.10 \\
(.60)\end{array}$ & $\begin{array}{l}4.28 \\
(.59)\end{array}$ & 3 & $\begin{array}{l}3.33 \\
(.91)\end{array}$ & $\begin{array}{l}3.57 \\
(.93)\end{array}$ & 2 \\
\hline Motivierungsqualität & $\begin{array}{l}1.53 \\
(.72)\end{array}$ & $\begin{array}{l}1.43 \\
(.53)\end{array}$ & 1 & $\begin{array}{l}3.78 \\
(.87)\end{array}$ & $\begin{array}{l}4.13 \\
(1.05)\end{array}$ & 3 & $\begin{array}{l}2.74 \\
(.79)\end{array}$ & $\begin{array}{l}3.14 \\
(.72)\end{array}$ & 2 \\
\hline $\begin{array}{l}\text { Diagnostik im } \\
\text { Leistungsbereich }\end{array}$ & $\begin{array}{l}2.01 \\
(.96)\end{array}$ & $\begin{array}{l}2.26 \\
(.81)\end{array}$ & 2 & $\begin{array}{l}3.62 \\
(.76)\end{array}$ & $\begin{array}{l}3.63 \\
(.95)\end{array}$ & 2 & $\begin{array}{l}3.18 \\
(.80)\end{array}$ & $\begin{array}{l}3.37 \\
(.84)\end{array}$ & 2 \\
\hline $\begin{array}{l}\text { Individuelle Bezugs- } \\
\text { normorientierung }\end{array}$ & $\begin{array}{l}1.76 \\
(.83)\end{array}$ & $\begin{array}{l}1.93 \\
(.85)\end{array}$ & 1 & $\begin{array}{l}3.48 \\
(.70)\end{array}$ & $\begin{array}{l}3.67 \\
(.99)\end{array}$ & $\begin{array}{l}2 \\
--\end{array}$ & $\begin{array}{l}3.35 \\
(.82)\end{array}$ & $\begin{array}{l}3.86 \\
(.81)\end{array}$ & $\begin{array}{l}1 \\
--\end{array}$ \\
\hline $\begin{array}{l}\text { Diagnostik im } \\
\text { sozialen Bereich }\end{array}$ & $\begin{array}{l}1.57 \\
(.76)\end{array}$ & $\begin{array}{l}1.43 \\
(.57)\end{array}$ & 1 & $\begin{array}{l}3.17 \\
(1.05)\end{array}$ & $\begin{array}{l}3.17 \\
(1.07)\end{array}$ & $\begin{array}{l}3 \\
\cdots\end{array}$ & $\begin{array}{l}2.89 \\
(.84)\end{array}$ & $\begin{array}{l}3.00 \\
(1.07)\end{array}$ & 3 \\
\hline Fürsorglichke it & $\begin{array}{l}1.63 \\
(.46)\end{array}$ & $\begin{array}{l}1.46 \\
(.43)\end{array}$ & 1 & $\begin{array}{l}3.64 \\
(.72)\end{array}$ & $\begin{array}{l}3.84 \\
(.72)\end{array}$ & 3 & $\begin{array}{l}3.01 \\
(.62)\end{array}$ & $\begin{array}{l}3.45 \\
(.68)\end{array}$ & 2 \\
\hline $\begin{array}{l}A M D \\
m \text { in } / \mathrm{m} \text { ax }\end{array}$ & $\begin{array}{r}0.15( \\
0.05(+) /\end{array}$ & $\begin{array}{l}(+/-) \\
0.25\end{array}$ & & $\begin{array}{r}0.16 \\
0.00 / 0\end{array}$ & $\begin{array}{l}(+) \\
35(+)\end{array}$ & & $0.11 \stackrel{0.33}{(+) /}$ & $\begin{array}{l}3(+) \\
/ 0.51(+)\end{array}$ & \\
\hline \multicolumn{10}{|c|}{ 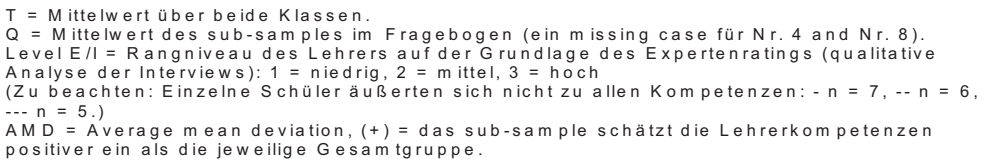 } \\
\hline
\end{tabular}

Um die Zusammenhänge zwischen Lehrerkompetenzen und Schüleremotionen auf der Ebene der Fallanalysen deskriptiv zu erfassen, wurden die drei Lehrkräfte anhand der Interviewaussagen charakterisiert (vgl. Tabelle 6).

Lehrperson 4 wurde beispielsweise in Bezug auf ihre Kompetenzen insgesamt auf einem niedrigen Niveau eingeschätzt. Insbesondere die Klarheit der Instruktion, die Motivierungsqualität sowie die Diagnostik im Leistungsbereich sind aus Sicht der Schülerinnen und Schüler niedrig ausgeprägt. Folgende Interviewaussagen (2) und (5) stützen die Annahme, dass Lehrperson 4 eher nicht daran interessiert ist, sich um einen guten Unterricht zu bemühen. Die zum Teil schon mit einem zynischen Unterton gefärbten Schüleraussagen (1) und (4) mit der expliziten Hassäußerung zeigen auf intra-individueller Ebene sehr deutlich, wie sich solche wahrgenommenen Defizite emotional auf Schülerinnen und Schüler auswirken können.

(1) „Ich denke, es ist die Hauptaufgabe einer Lehrperson, den Schülern alles zu erklären. Lehrperson Nr.4 macht es aber nie. Zudem verstehen wir nichts, was Nr. 4 erklärt. Manchmal sagt Nr. 4 auch, dass es jetzt besser für uns wäre, wir würden es verstehen."

(2) „Ich habe den Eindruck, dass Nr. 4 ihren Job nicht sehr ernst nimmt.”

(3) „Nr. 4 ist genau die Art von Lehrperson, die nicht mit Schülern reden. Ich hasse Nr. 4.” 
(4) „Nr. 4 lobt uns nie. Wenn Nr. 4 denkt, wir haben es verstanden, macht Nr. 4 sofort mit dem Stoff weiter."

(5) „Uns motivieren? Ich glaube, Nr. 4 will gar nicht, dass uns etwas interessiert und wir Spaß haben.” (Gläser-Zikuda/Fuß 2008: 129).

Tabelle 6: Interviewaussagen von Schülerinnen und Schülern zu den Kompetenzen der drei ausgewählten Lehrpersonen. Die Zitate wurden zum Teil so abgeändert, dass das Geschlecht der Lehrpersonen nicht erkennbar ist. (Gläser-Zikuda/Fuß 2008: 131)

\begin{tabular}{|c|c|c|}
\hline Lehrperson Nr. 4 (O) & Lehrperson Nr. 6 (๑) & Lehrperson Nr. 8 (X) \\
\hline $\begin{array}{l}\text { (1) „Ich denke, es ist die } \\
\text { Hauptaufgabe einer } \\
\text { Lehrperson, den Schülern } \\
\text { alles zu erklären. Nr. } 4 \\
\text { macht es aber nie. Zudem } \\
\text { verstehen wir nichts, was } \\
\text { Nr. } 4 \text { erklärt. Manchmal } \\
\text { sagt Nr. } 4 \text { auch, dass es } \\
\text { jetzt besser für uns wäre, } \\
\text { wir würden es verstehen.“ } \\
\text { (2) „Ich habe den } \\
\text { Eindruck, dass Nr. } 4 \text { ihren } \\
\text { Job nicht sehr ernst } \\
\text { nimmt.“ } \\
\text { (3), Nr. } 4 \text { ist genau die } \\
\text { Art von Lehrperson, die } \\
\text { nicht mit Schülern redet } \\
\text { Ich hasse Nr. } 4 . “ \\
\text { (4) „Nr. } 4 \text { lobt uns nie. } \\
\text { Wenn Nr. } 4 \text { denkt, wir } \\
\text { haben es verstanden, } \\
\text { macht Nr. } 4 \text { sofort mit } \\
\text { dem Stoff weiter.“ } \\
\text { (5) „Uns motivieren? Ich } \\
\text { glaube, Nr. } 4 \text { will gar } \\
\text { nicht, dass uns etwas } \\
\text { interessiert und wir Spaß } \\
\text { haben.“ }\end{array}$ & $\begin{array}{l}\text { (1) „Wir mögen Nr. } 6 \\
\text { total. Nr. } 6 \text { hat echt eine } \\
\text { gute Beziehung zu uns } \\
\text { allen.“" } \\
\text { (2) „Nr. } 6 \text { ist wirklich } \\
\text { Schülers } \\
\text { Lieblingslehrperson.“ } \\
\text { (3) „Nr. } 6 \text { macht oft } \\
\text { Witze und erzählt uns } \\
\text { sogar, wie sie selbst } \\
\text { als Schüler Lehrer } \\
\text { geärgert hat.“ } \\
\text { (4) „Nr. } 6 \text { erklärt einen } \\
\text { Inhalt sofort und gut, } \\
\text { wenn ein Schüler } \\
\text { Probleme hat, es zu } \\
\text { verstehen.“ } \\
\text { (5) „Nr. } 6 \text { kommt ins } \\
\text { Klassenzimmer und das } \\
\text { Erste, was sie macht, ist } \\
\text { uns darüber zu } \\
\text { informieren, was wir } \\
\text { heute lernen werden.“ }\end{array}$ & $\begin{array}{l}\text { (1) „Normalerweise ist } \\
\text { der Unterricht bei Nr. } 8 \\
\text { interessant und manchmal } \\
\text { sogar spannend.“" } \\
\text { (2) „Nr. } 8 \text { ist zwar schon } \\
\text { XX Jahre alt, aber Nr. } 8 \\
\text { ist sportlich und } \\
\text { unterrichtet wie eine } \\
\text { junge Lehrperson.“" } \\
\text { (3) „Nr. } 8 \text { hilft schon, } \\
\text { wenn ein Schüler bei } \\
\text { einer Aufgabe nicht } \\
\text { weiter weiß.“ } \\
\text { (4) „Nr. } 8 \text { fragt eigentlich } \\
\text { nicht, ob wir die Stunde } \\
\text { jetzt gut fanden.““ }\end{array}$ \\
\hline
\end{tabular}

Die Nützlichkeit der Verbindung quantitativer und qualitativer Methoden konnte in dieser Studie durch Kreuzvalidierungen demonstriert werden. Zunächst konnte durch den rein quantitativen Vergleich der beiden Schulklassenkennwerte der drei Lehrpersonen mit den Kennwerten der drei Interviewstichproben im Sinne des theoretical sampling gezeigt werden, dass die Interviewstichproben weitgehend als repräsentativ für die Beschreibung und Einschätzung der Lehrerkompetenzen angesehen werden können. Die Kombination der qualitativen und 
quantitativen Forschungsmethoden zu Validierungszwecken erwies sich als sehr günstig. Darüber hinaus war es auch möglich, mehrere Perspektiven auf den Untersuchungsgegenstand richten zu können. Die Schüler-, die Lehrerperspektive sowie die auf den Schülerinterviews basierende Expertensicht wurden berücksichtigt. Alle drei Zugänge stützen die Annahme, dass Lehrerkompetenzen eine wichtige Rolle für das emotionale Erleben der Schülerinnen und Schüler spielen und damit auch einen Einfluss auf deren Lernprozess haben.

Im heutigen Forschungsalltag ist es durchaus üblich, sowohl qualitative als auch quantitative Methoden kombiniert einzusetzen (Hofmann et al. 2008). Ob ein Forschender quantitativ, qualitativ vorgeht oder beide Forschungsmethoden kombinierend einsetzt, ist letztendlich vom Forschungsgegenstand abhängig und nicht von einer dogmatischen Ausrichtung im Hinblick auf eines der beiden Paradigmen. Wolf (1995) argumentiert, dass sich die beiden Paradigmen im Forschungsalltag ohnehin nicht trennen lassen. So hat eine quantitative Forschung immer einen qualitativen Start und ein ebensolches Ende:

Vor einer quantitativen Datensammlung muss der Forscher, will er nicht Beliebiges produzieren, in einem Interpretationsprozess Sinn und Reichweite seiner Untersuchungsfragestellung begründen, und am Ende einer quantitativen Datenaufbereitung stehen immer

Zahlen in Form statistischer Parameter, die es zu interpretieren gilt. (Wolf 1995: 319-320)

Qualitative Untersuchungen sind häufig deskriptiv und interpretierend, aber sie schließen auch beispielsweise mit einer Typenbildung ab, was quantitativ gesehen bedeutet, dass eine Variable mit nominalem Messniveau gewonnen wurde (Wolf 1995).

\section{Computereinsatz bei qualitativen Auswertungsverfahren}

Abschließend soll kurz auch auf computerunterstützte Möglichkeiten der Datenanalyse eingegangen werden, durch die in den letzten 15 Jahren die Durchführung qualitativer Forschungsprojekte sehr erleichtert worden ist. Das heißt nicht, dass ein Computerprogramm qualitativ Forschenden die kreative Arbeit des Sinnverstehens und der Textinterpretation abnehmen könnte, wohl aber kann entsprechende Software eine große Hilfe bei der Dokumentation und Speicherung von Kodierungen, beim Ordnen der Daten sowie beim Visualisieren von analytischen Strukturen sein.

Hauptziel qualitativer Analyse ist es, die normalerweise wörtlichen Beschreibungen, Erklärungen, Beobachtungsprotokolle, Interviews, Videoaufzeichnungen usw. so zu reduzieren, dass die ursprünglichen Daten zu systematischen Aussagen über ihre Bedeutung zusammengefasst werden können. Die Art und Weise der Analyse hängt letztlich von der Fragestellung einer bestimmten Studie ab. In fast allen qualitativen Analysen spielt die Klassifikation oder Kategorisierung von Daten eine besondere Rolle. Entweder werden die Daten 
deduktiv nach einem vorgegebenen, in der Regel theoretisch abgeleiteten Kategoriensystem zugeordnet. Oder aber die Kategorien werden auf der Grundlage zentraler Forschungsfragen, Hypothesen oder wichtiger inhaltlicher Aspekte induktiv entwickelt.

Qualitatives Analysieren und Kodieren soll im Folgenden an zwei Beispielen näher erläutert werden, und zwar zunächst am Beispiel der Software AQUAD und dann am Beispiel von ATLAS.ti.

\subsection{Die Auswertungssoftware AQUAD}

Diese Software zur Auswertung qualitativer Daten AQUAD (Huber 1992, Huber/ Gürtler 2003) wurde dafür entwickelt, die Rekonstruktion von Bedeutungszusammenhängen im Datenmaterial zu unterstützen, angelehnt beispielsweise an den methodologischen Ansatz der Grounded Theory (Glaser/Strauss 1965).

AQUAD unterstützt sowohl den deduktiven wie den induktiven Analyseprozess sowie eine Kombination aus beiden. Im Unterschied zu vielen anderen Programmen, die zur Unterstützung qualitativer Analyse entwickelt wurden, ist AQUAD bei der Inhaltsanalyse von Transkripten, Beobachtungsprotokollen oder anderen Texten hilfreich. Es kann zum einen nach Worten und Sätzen in den Daten gesucht und es können Häufigkeiten ausgezählt werden. Zum anderen lassen sich auch Wörter zusammen mit dem jeweiligen Kontext heraussuchen. Weiterhin ermöglicht es AQUAD, mit Hilfe von Memos Kommentare mit Kodes oder beliebigen Datensegmenten zu verknüpfen. Mit Hilfe von Memos (Glaser/Strauss 1965) kann der Forschende festhalten, was ihm/ihr zu den Kodes und zum Kodieren, über Zusammenhänge, Widersprüche, Ausnahmen usw. einfällt. Diese Notizen lassen sich mit der Nummer der Datei, der Positionsnummer eines relevanten Segments sowie dem Kode verknüpfen (Huber/Gürtler 2003).

Die neueren Versionen von AQUAD ermöglichen qualitative Analysen ohne aufwändige Transkriptionen. Neben transkribierten Interviews können auch Tonaufzeichnungen als Audiodateien, Videoaufzeichnungen sowie Bilddateien direkt in AQUAD analysiert werden.

Das Programm ist insbesondere für die Generierung von Theorien auf der Basis qualitativer Daten entwickelt worden und legt besonderen Wert auf die Gütekriterien der Objektivität, Reliabilität und Validität (ebd.).

\subsection{Die Auswertungssoftware ATLAS.ti}

Ein Prototyp von ATLAS.ti wurde von Thomas Muhr an der Technischen Universität Berlin von 1989 bis 1992 entwickelt. Die erste kommerzielle Version von ATLAS.ti kam 1993 auf den Markt (vgl. http://www.atlasti.com/de/). Vor- 
aussetzung für die computerunterstützte qualitative Datenanalyse mit ATLAS.ti ist, dass die Ausgangsdaten oder Primärdokumente (Interviews oder andere Texte, aber auch Bildmaterial und Multimedia) auf einer Festplatte als Dateien gespeichert sind. Zu Beginn werden alle für eine Forschungsfrage bedeutsamen Dokumente in einer sogenannten ,hermeneutischen Einheit" (hermeneutic unit $=$ HU) zusammengefasst (vgl. Friese 2011, Strübing 1997). Auf der Ebene der Primärdokumente (textuelle Ebene) werden bedeutsame Text- oder Bildausschnitte markiert und mit Kodes oder Kommentaren versehen. Die Kodierung erfolgt entweder aufgrund der interpretierenden Verstehensleistung des Forschers oder halbautomatisch (d.h. die Suche nach bestimmten vorab theoretisch festgelegten Wörtern oder Wortkombinationen im Text - ähnlich wie bei der strukturierenden Inhaltsanalyse). Auf der konzeptuellen Ebene stehen Werkzeuge zur Verfügung, um die Kodes (Schlagwörter, Konzepte) miteinander zu vernetzen, die erzeugten Relationen zu benennen und Begriffsnetze bzw. Modelle zu visualisieren. Die so erzeugten Modelle erlauben es, von zusammenfassenden und abstrahierenden Konzepten aus bei Bedarf auf die ihnen zugrunde liegenden Textpassagen zurückzugreifen und sie in ihrem Kontext zu vergleichen. Die Arbeit mit ATLAS.ti trägt nicht zuletzt erheblich zur Qualitätssteigerung im Umgang mit den Daten bei, weil es den gesamten Prozess der Kodierung und das Erstellen von Beziehungen zwischen Sinneinheiten dokumentiert und der Prozess dadurch einer Rekonstruktion zugänglich wird.

\section{$5 \quad$ Fazit}

Wie deutlich geworden sein dürfte, müssen bei qualitativen Auswertungsverfahren, wie das Beispiel der Qualitativen Inhaltsanalyse zeigt, zahlreiche Schritte bedacht werden. Beginnend mit der Forschungsfrage müssen Forschende über die Wahl geeigneter Erhebungsmethoden, die Aufbereitung der Daten, über die Auswahl der Analysetechniken, die Entwicklung eines Kodierleitfadens bis hin zur Überprüfung von Gütekriterien alle einzelnen Schritte gründlich überlegen und begründet entscheiden. Nur aus der Fragestellung, dem eigentlichen Ziel der Analyse, lässt sich letztlich ableiten, wie das Datenmaterial konkret ausgewertet werden soll.

Wie anhand der zahlreichen Beispiele illustriert wurde, bietet die Qualitative Inhaltsanalyse hierfür verschiedene Techniken an: induktive Kategorienbildung bzw. zusammenfassende Inhaltsanalyse, Explikation (enge und weite Kontextanalyse) sowie deduktive Kategorienanwendung bzw. Strukturierung (formale, inhaltliche, typisierende und skalierende Strukturierung).

Je nach Forschungsfrage und Teilaspekten des Forschungsgegenstandes ist auch eine Kombination der verschiedenen Techniken möglich. In diesen Auswertungstechniken spielen qualitative und quantitative Analyseschritte jeweils 
eine spezifische Rolle. Hierin zeigt sich die Nähe der Qualitativen Inhaltsanalyse zu Mixed Methods-Ansätzen. Schlussendlich kann damit aber auch verdeutlicht werden, wie überholt eine Dichotomisierung quantitativer versus qualitativer Forschungsmethodik ist. Die Qualitative Inhaltsanalyse als weit verbreitete und etablierte Auswertungsmethode für qualitative Daten bietet jedenfalls vielfältige Analysemöglichkeiten für die Bildungsforschung.

\section{Literatur}

ATLASti. Qualitative data analysis.Online: http://www.atlasti.com/de/ (letzter Aufruf [22.02.12])

Aufschnaiter, Stefan v. / Welzel, Manuela (2001). Nutzung von Videodaten zur Untersuchung von Lehr-Lern-Prozessen. Münster: Waxmann.

Breidenstein, Georg (2008). Allgemeine Didaktik und praxeologische Unterrichtsforschung. Meyer, Meinert A. / Prenzel, Manfred / Hellekamps, Stephanie (Hrsg.): Perspektiven der Didaktik. Zeitschrift für Erziehungswissenschaft. Sonderheft 9: 201-215.

Combe, Arno / Helsper, Werner / Stelmaszyk, Bernd (Hrsg.) (1999). Forum qualitative Schulforschung. Band I. Weinheim: Deutscher Studien Verlag.

Flick, Uwe (2004). Triangulation. Eine Einführung. Wiesbaden: VS Verlag für Sozialwissenschaften.

Flick, Uwe / Kardorff, Ernst v. / Steinke, Ines (Hrsg.) (2008). Qualitative Forschung - Ein Handbuch. Reinbek: Rowohlt, 6. Auflage.

Friebertshäuser, Barbara / Langer, Antje / Prengel, Annedore (Hrsg.) (2010). Handbuch qualitative Forschungsmethoden in der Erziehungswissenschaft. Weinheim: Juventa, 3. Auflage.

Friese, Susanne (2011). Using ATLAS.ti for analyzing the financial crisis data [67 paragraphs]. In: Forum Qualitative Sozialforschung / Forum: Qualitative Social Research, 12(1), Art. 39. Online: nbn-resolving.de/urn:nbn:de:0114-fqs1101397 (letzter Aufruf [23.02.12]).

Gläser-Zikuda, Michaela (2001). Emotionen und Lernstrategien in der Schule. Eine empirische Studie mit Qualitativer Inhaltsanalyse. Weinheim: Beltz/DSV.

Gläser-Zikuda, Michaela / Fuß, Stefan (2008). Lehrerkompetenzen und Schüleremotionen. Wie nehmen Lernende ihre Lehrkräfte emotional wahr? In: Gläser-Zikuda, Michaela/ Seifried, Jürgen (Hrsg.): Lehrerexpertise - Analyse und Bedeutung unterrichtlichen Handelns. Münster: Waxmann. 113-144.

Gläser-Zikuda, Michaela / Lindacher, Tanja / Fuß, Stefan (2006). Wirksamkeit eines Portfolios zur Förderung von Lernleistungen und Lernstrategien - eine quasi-experimentelle Studie. Empirische Pädagogik 20/3: 229-244.

Gläser-Zikuda, Michaela / Seidel, Tina / Rohlfs, Carsten / Gröschner, Alexander / Ziegelbauer, Sascha (Hrsg.) (2012). Mixed Methods in der empirischen Bildungsforschung. Münster: Waxmann

Glaser, Bernard G. / Strauss, Anselm L. (1965). The discovery of grounded theory. Strategies for qualitative research. Chicago: Aldine Publishing Company. 
Haudeck, Helga (2008). Wie pauken Schülerinnen und Schüler Vokabeln für den Fremdsprachenunterricht wirklich? In: Mayring, Philipp / Gläser-Zikuda, Michaela (Hrsg.): Die Praxis der Qualitativen Inhaltsanalyse. Weinheim: Beltz. 84-104. 2. Auflage.

Helsper, Werner / Böhme, Jeanette (Hrsg.) (2008). Handbuch der Schulforschung. Wiesbaden: VS Verlag für Sozialwissenschaften.

Hofmann, Franz / Schreiner, Claudia / Thonhauser, Josef (Hrsg.) (2008). Qualitative und quantitative Aspekte. Zu ihrer Komplementarität in der erziehungswissenschaftlichen Forschung. Münster: Waxmann.

Huber, Günter L. (1992). Qualitative Analyse mit Computerunterstützung. In: Huber, Günter L. (Hrsg.): Qualitative Analyse. Computereinsatz in der Sozialforschung. München: Oldenbourg. 115-176.

Huber, Günter L. / Gürtler, Leo (2003). AQUAD 6. Manual zur Software AQUAD 6. Tübingen: Huber.

Krippendorff, Klaus (2004). Content analysis: An introduction to its methodology. 2nd edition. Thousand Oaks, CA: Sage.

Lamnek, Stefan (1993). Qualitative Sozialforschung. Weinheim: PVU.

Lindacher, Tanja (2005). Das Portfolio im Unterricht. Eine Interventionsstudie. Unveröffentlichte Diplomarbeit. Pädagogische Hochschule Ludwigsburg.

Mayring, Philipp (2001). Kombination und Integration qualitativer und quantitativer Analyse [Combination and integration of qualitative and quantitative analysis]. Forum Qualitative Sozialforschung / Forum: Qualitative Social Research, 2 (1). Online: http://www.qualitativeresearch.net/fqs (letzter Aufruf [23.02.12])

Mayring, Philipp (2002). Einführung in die qualitative Sozialforschung. Weinheim: Beltz Studium, 5. Auflage.

Mayring, Philipp (2008). Qualitative Inhaltsanalyse. Grundlagen und Techniken. Weinheim: Beltz, 10. Auflage.

Mayring, Philipp / Gläser-Zikuda, Michaela (Hrsg.) (2008). Die Praxis der Qualitativen Inhaltsanalyse. Weinheim: Beltz, 2. Auflage.

Mey, Günter / Mruck, Katja (2010). Handbuch qualitative Forschung in der Psychologie. Wiesbaden: VS Verlag für Sozialwissenschaften.

Reinders, Heinz / Ditton, Hartmut / Gräsel, Cornelia / Gniewosz, Bernd (Hrsg.) (2010). Empirische Bildungsforschung. Strukturen und Methoden. Wiesbaden: VS Verlag für Sozialwissenschaft.

Seidel, Tina (2011). Lehrerhandeln im Unterricht. In: Terhart, Ewald / Bennewitz, Hedda / Rothland, Martin (Hrsg.): Handbuch der Forschung zum Lehrerberuf. Münster: Waxmann, 605-629.

Steinmetz, Michael (2011). Realisierbarkeit als Merkmal guter Bildungsstandards für das Abitur. Eine qualitativ-empirische Studie. Dissertationsschrift. Friedrich-Schiller-Universität Jena.

Strübing, Jörg (1997). ATLAS/ti-Kurs. Einführung in das Arbeiten mit dem Programm ATLAS/ti für Windows 95 Versionen 4.0 und 4.1. Mitteilungen aus dem Schwerpunktbereich Methodenlehre, Heft 48. Institut für Soziologie, Freie Universität Berlin. 
Tashakkori, Abbas / Teddlie, Charles (1998). Mixed methodology: combining qualitative and quantitative approaches. Thousand Oaks, CA: Sage.

Tashakkori, Abbas / Teddlie, Charles (eds.) (2003). Handbook of mixed methods in social and behavioral research. Thousand Oaks, CA: Sage.

Wirtz, Markus / Caspar, Franz (2002). Beurteilerübereinstimmung und Beurteilerreliabilität. München: Hogrefe.

Wolf, Willi (1995). Qualitative vs. Quantitative Forschung. In: König, Eckard / Zedler, Peter (Hrsg.): Bilanz qualitativer Forschung. Grundlagen qualitativer Forschung. Weinheim: DSV. Bd. 1. 309-329. 


\title{
Die qualitative Inhaltsanalyse als Interpretationsverfahren zur Generierung eines empirischen Modells inter- kultureller Kompetenz
}

\author{
Jan-Oliver Eberhardt
}

Intercultural competence is seen as an important objective of foreign language learning. However, there is a lack of theoretically and empirically sound models which contain adequate intercultural educational objectives with regard to students in secondary schools. In this article, I will report on a qualitative study with German tenth-graders learning French as a foreign language in which the pupils had to assess critical incidents. While the focus of the article is on the data analysis based on the standard procedures and techniques of Qualitative Content Analysis (QCA), the methodology and design of the study will also be presented. The paper concludes with a short critical discussion of the QCA.

\section{Einleitung}

Bereits in der Expertise zur Entwicklung nationaler Bildungsstandards wurde deutlich, dass ein outputorientierter Schulunterricht die curriculare Implementierung domänenspezifischer Kompetenzmodelle voraussetzt:

Bildungsstandards (...) stützen sich auf Kompetenzmodelle, die in Zusammenarbeit von Pädagogik, Psychologie und Fachdidaktik entwickelt werden müssen. Ein solches Kompetenzmodell unterscheidet Teildimensionen innerhalb einer Domäne (...), und es beschreibt jeweils unterschiedliche Niveaustufen auf solchen Dimensionen. (...). Zum Bildungsstandard gehört, dass für einzelne Jahrgänge festgelegt wird, welche Stufen die Schülerinnen und Schüler erreichen sollen. (Klieme 2003: 22)

Präzise Kompetenzdeskriptoren sind in erster Linie für die Konstruktion von Lern- und Testaufgaben, welche die Förderung und Überprüfung der zu erreichenden fachspezifischen Kompetenzen zum Ziel haben, unabdingbar. Die Feststellung, dass die für die Domäne fremdsprachlichen Lernens konzipierten Bildungsstandards für die erste Fremdsprache für den Mittleren Schulabschluss (Englisch/Französisch) (KMK 2003) ausschließlich im Bereich der kommunikativen Fertigkeiten sowie der Verfügung sprachlicher Mittel differenzierte Kompetenzformulierungen aufweisen, muss deshalb äußerst kritisch betrachtet werden. Nicht wenige mit der Erforschung des Lehrens und Lernens fremder Sprachen befasste Wissenschaftler ${ }^{1}$ sehen ganz besonders im Fehlen einer Dimensionierung und Graduierung interkultureller Kompetenzen die Gefahr, einen in Anbetracht des fortschreitenden Globalisierungsprozesses sowie der

\footnotetext{
${ }^{1}$ Im Sinne der Lesbarkeit verwende ich im gesamten Text die maskuline Form von Personenbezeichnungen, in denen jedoch auch weibliche Personen mit inbegriffen sind.
} 
migrationsbedingten Zunahme sprachlich-kulturell heterogener Gesellschaften unverzichtbaren Kernbereich des Fremdsprachenunterrichts zu vernachlässigen (Bausch et al. 2005, Eberhardt 2008, 2009, 2010, Hu 2008). Als ein erster Beleg für diese Befürchtung kann zweifelsohne die Tatsache gewertet werden, dass bis zum heutigen Tag sowohl in den länderspezifischen Vergleichsarbeiten als auch in den vom IQB (Institut zur Qualitätsentwicklung im Bildungswesen) entwickelten Testaufgaben ausschließlich die funktional-kommunikativen Kompetenzen Berücksichtigung finden (Tesch et al. 2008). Als Ursache für die nicht erfolgte Operationalisierung interkultureller Fähig- und Fertigkeiten wird unisono die Komplexität dieses Kompetenzbereichs angeführt. Weit verbreitet innerhalb der Fremdsprachendidaktik sind zudem standardkritische Diskurse, welche interkulturelle, reflexive, ethische und ästhetische Aspekte sprachlichen Lernens per se als unvereinbar mit den Prinzipien der Kompetenzorientierung erachten (Bredella/Christ 2007, Hu 2008). Um zu verhindern, dass interkulturelle Kompetenzen dauerhaft ein Schattendasein im standardbasierten Fremdsprachenunterricht führen, wird es allerdings nicht genügen, lediglich auf die gebührende Berücksichtigung dieses Kompetenzbereichs seitens der Schulen und Fachlehrer zu hoffen. Erst die Bereitstellung eines auf horizontaler wie vertikaler Ebene ausdifferenzierten Modells interkultureller Kompetenz für die Bildungsstandards - und damit auch für die Kerncurricula der einzelnen Bundesländer - schafft die hierfür erforderliche Verbindlichkeit und ermöglicht zugleich den Einsatz bisher fehlender Lern- und Testaufgaben. Ohne Frage hält dieses Szenario massive konzeptuelle wie empirische Herausforderungen bereit, denen die fremdsprachendidaktische Forschung nur in Zusammenarbeit mit ihren Bezugsdisziplinen begegnen können wird (Hu/Byram 2009, Zydatiß 2006).

Der vorliegende Beitrag gewährt Einblick in ein empirisches Forschungsprojekt, das das Ziel der Konzipierung eines interkulturellen Kompetenzmodells für den Fremdsprachenunterricht der Sekundarstufe I verfolgt und einen ersten entscheidenden Schritt auf dem damit verbundenen Weg begeht. Im Zentrum dieses Projekts steht die empirische Ausdifferenzierung eines theoretisch konzipierten Strukturmodells interkultureller Kompetenz mittels qualitativer Inhaltsanalyse nach der Version von Mayring (2003). Im folgenden Kapitel werde ich zunächst kurz auf bereits existierende wissenschaftliche Modellierungen interkultureller Kompetenz eingehen, um dann im Anschluss (Kapitel 2) das Forschungsinteresse und das Design meines Dissertationsprojekts vorzustellen. Meine besondere Konzentration wird im Zuge dessen der hier gewählten Auswertungsmethode, sprich der qualitativen Inhaltsanalyse, gelten. In Kapitel 3 sollen schließlich die konkreten Abläufe des Interpretationsprozesses nachvollziehbar gemacht werden. Mit einer kurzen Diskussion der Kritikpunkte an einer inhaltsanalytischen Datenauswertung wird dieser Beitrag schließen. 


\section{Wissenschaftliche Modellierungen interkultureller Kompetenz}

Innerhalb der Bildungswissenschaften besteht darüber Einigkeit, dass erst auf Grundlage empirisch überprüfter Kompetenzmodelle valide Testinstrumente zum Zwecke des Bildungsmonitoring, der Evaluation von Schulen und der Individualdiagnostik konstruiert werden können (Aguado et al. 2010, Grünewald/Küster 2009, Hu/Byram 2009, Klieme/Leutner 2006, Köller 2008). Laut Klieme (2003) müssen fachspezifische Kompetenzmodelle neben einer horizontalen und vertikalen Aufschlüsselung auch auf die Domäne des Fremdsprachenunterrichts zugeschnitten sein sowie auf dem Forschungsstand der jeweiligen Fachdidaktik basieren. Die Sichtung von fremdsprachendidaktischen und bezugswissenschaftlichen Diskursen interkulturellen Lernens verdeutlicht, dass die bereits existierenden Modellierungen interkultureller Kompetenz ebenso wenig den Anforderungen eines standardorientierten Fremdsprachenunterrichts gerecht werden wie die aktuellen Kompetenzdeskriptoren der Bildungsstandards. Zwar haben insbesondere die Kommunikationswissenschaften sowie die Sozial- und die kulturvergleichende Psychologie eine nahezu unüberschaubare Fülle an konzeptuell wie empirisch konstruierten Kompetenzmodellen hervorgebracht (Eberhardt 2008, Hu/Byram 2009), diese jedoch nicht auf den Fremdsprachenunterricht, sondern in erster Linie auf die Anwendungskontexte internationaler Austausch und interkulturelles Training bezogen (Bennett 1986, Bolten 2007, Chen/Starosta 2005, Thomas 2003). Anders verhält es sich dagegen mit dem explizit für das institutionelle Lehren und Lernen fremder Sprachen entwickelten Strukturmodell interkultureller Kompetenz des Fremdsprachendidaktikers Byram (1997).

Byram untergliedert die horizontale Dimension der interkulturellen Kompetenz in fünf Teilkomponenten, in die sogenannten savoirs. Dabei bilden kulturspezifisches Wissen über eigene und fremde Kulturen sowie kulturallgemeines Wissen über persönliche und gesellschaftliche Interaktionsprozesse die Wissenskomponente interkultureller Kompetenz (savoirs). Eine weitere Komponente beinhaltet Einstellungen wie Neugier, Offenheit sowie die Bereitschaft, eigene Überzeugungen zu relativieren (savoir être). Des Weiteren spricht Byram interkulturell kompetenten Menschen die Fähigkeit zu, Dokumente oder Ereignisse anderer Kulturen $\mathrm{zu}$ interpretieren, zu erklären und miteinander in Beziehung zu setzen (savoir comprendre). Ebenso wird die Fähigkeit, neues Wissen über andere Kulturen zu erwerben und die Bewältigung interkultureller Begegnungssituationen als Bestandteil interkultureller Kompetenz erachtet (savoir apprendre(faire). Die fünfte Teilkompetenz, savoir s'engager, impliziert den kritischen Umgang mit kulturellen Produkten und Praktiken auf Basis expliziter Kriterien. Jene horizontale Aufschlüsselung interkultureller Kompetenz hat Byram zudem in Teillernziele ausdifferenziert, welche aufgrund ihrer can do- 
Formulierungen stark den Deskriptoren der kommunikativen Teilkompetenzen im Gemeinsamen europäischen Referenzrahmen für Sprachen (Goethe-Institut et al. 2001) ähneln. Sein Strukturmodell basiert obendrein auf zahlreichen theoretischen Standpunkten der Didaktik des Fremdverstehens (Bredella/Christ 2007), wie beispielsweise auf der Berücksichtigung des Zusammenhangs zwischen kommunikativen und interkulturellen Kompetenzen, zwischen Sprache und Kultur sowie auf der Abgrenzung von binational ausgerichteten interkulturellen Lernprozessen.

Ungeachtet seiner hier nur verkürzt aufgezeigten Tauglichkeit offenbart Byrams Modell dennoch drei fundamentale Defizite. Erstens mangelt es an einer vertikalen Kompetenzstufung. Zweitens erscheinen die Teilaspekte interkultureller Kompetenz nach wie vor in nur unzureichendem Maße ausdifferenziert; obendrein unterliegen sie einem relativ hohen Abstraktionsniveau. Auf Grundlage dieser Erkenntnisse könnte die Ausdifferenzierung von Byrams Strukturmodell interkultureller Kompetenz einen entscheidenden Beitrag auf dem Weg der Entwicklung eines Kompetenzmodells für die Bildungsstandards darstellen. Angesichts der bis dato bestehenden Unklarheit darüber, welche Kompetenzanforderungen für Französischlerner am Ende der Jahrgangsstufe 10 entwicklungsangemessen sind (Eberhardt 2010, Hu/Byram 2009) sowie unter Berücksichtigung der bildungswissenschaftlichen Forderung nach theoretisch wie empirisch fundierten Kompetenzmodellen, welche „die Perspektive der Lernenden in den Mittelpunkt rücken“ (Rehm 2006: 26), empfiehlt es sich meines Erachtens, die angestrebte Ausdifferenzierung auf empirisch gewonnene Daten zu gründen. Genau hier setzt mein im folgenden Kapitel behandeltes Forschungsprojekt an.

\section{Studie zur empirischen Ausdifferenzierung eines Struktur- modells interkultureller Kompetenz}

Die Ausdifferenzierung eines theoretisch konzipierten Kompetenzmodells anhand empirischer Daten legt eine qualitative Vorgehensweise nahe, welche dem Forschungsgegenstand mit Offenheit begegnet, bisher unbekannte Aspekte exploriert oder bereits bestehende Hypothesen modifiziert (Flick 2005, Lamnek 2005). Hierfür ist ein offenes, nicht-standardisiertes Erhebungsinstrument erforderlich, welches die Untersuchungspersonen mit holistischen, interkulturelle Fähig- und Fertigkeiten ansprechenden Aufgaben konfrontiert (Eberhardt 2010). Zum Zwecke des theoretischen Anschlusses an die Bildungsstandards sowie der Entwicklungsangemessenheit curricularer Lernziele wurden als Untersuchungspopulation Schüler der Jahrgangsstufe 10 bestimmt. Die Auswahl der Schüler erfolgte über ein gezieltes Sampling, um das Untersuchungsfeld in seiner ganzen Heterogenität erfassen und dadurch die Generalisierbarkeit der Ergebnisse erhöhen zu können (Flick 2005). Im Kontext des Französischunterrichts wurden 
zunächst N=106 Zehntklässler an Gymnasien und Gesamtschulen einer norddeutschen Großstadt per offenem Fragebogen untersucht und im Anschluss daran - unter Beachtung eines zentralen Gütekriteriums qualitativer Sozialforschung, der Daten- und Methodentriangulation ${ }^{2}$ (Lamnek 2005) - N=15 Französischlerner mittels vertiefender fokussierter Interviews befragt. Orientiert an gängigen Methoden der Exploration und der qualitativen Messung interkultureller Kompetenz (Bolten 2007, Hu/Byram 2009, Sercu 2004) ging es bei der schriftlichen Befragung um die Kommentierung und Beurteilung kulturell aufgeladener Fotos und interkultureller Dilemmata sowie um Erzählungen über eigene Erfahrungen mit Menschen anderer Kultur und Sprache, in der mündlichen Befragung fungierten Filmausschnitte mit interkulturellen Begegnungssituationen als Gesprächsstimulus. Der thematische Fokus richtete sich jeweils auf die französische Sprache und frankophone Länder (Eberhardt 2010).

Die aus der Befragung hervorgegangenen Reflexionen der Französischlerner wurden zunächst im Hinblick auf die dabei zum Vorschein kommenden horizontalen Dimensionen interkultureller Kompetenz analysiert. Anschließend galt es, die gewonnenen Teilaspekte interkultureller Kompetenz auf unterschiedliche Kompetenzniveaus hin zu untersuchen. In Entsprechung zu den forschungsmethodologischen und -methodischen Prinzipien des qualitativen Paradigmas ging es bei diesen Analysen stets um ein Kompetenzverstehen durch die Interpretation von Sprache und Erfahrung (Erpenbeck/von Rosenstiel 2003).

Als kodierendes, auf die Reduktion von Material und die Entwicklung von Kategorien abzielendes Interpretationsverfahren sah ich speziell mit der im vorliegenden Projekt eingesetzten qualitativen Inhaltsanalyse nach Auslegung von Mayring (2003) das Potenzial verbunden, aus einer großen Stichprobe kategorienhafte Teilaspekte interkultureller Kompetenz herauszuarbeiten. Nebenbei trägt die Inhaltsanalyse auch den, im Vergleich zu narrativen Interviews eher kleinen Textpassagen der offenen Fragebögen und fokussierten Interviews Rechnung und steht damit in Opposition zu sequenziellen Verfahren der Dateninterpretation, welche der Gestalt des Textes eine große Bedeutung beimessen und vorzugsweise auf Erzählungen angewendet werden (Flick 2005, Gläser/ Laudel 2006, Lamnek 2005). Obendrein bietet ein inhaltsanalytisches Vorgehen im Gegensatz zu anderen qualitativen Interpretationsverfahren die Möglichkeit, die Explorierung eines Untersuchungsgegenstandes mit einer - im Grunde genommen mit dem qualitativen Paradigma unvereinbaren - Falsifizierung bereits existierender Hintergrundtheorien zu kombinieren und „den scheinbaren Widerspruch zwischen dem Prinzip des theoriegeleiteten Vorgehens und dem Prinzip der Offenheit zu lösen“ (Gläser/Laudel 2006: 199). Die Verbindung

\footnotetext{
${ }^{2}$ Neben der Triangulation stuft Lamnek (2005) die Verfahrensdokumentation, die Nähe zum Gegenstand, die kommunikative Validierung, eine argumentative Interpretationsleistung und die Regelgeleitetheit als Gütekriterien qualitativer Sozialforschung ein.
} 
einer offenen induktiven und theoriegeleiteten deduktiven Erkenntnisgewinnung ist im Hinblick auf mein Forschungsinteresse - die empirische Modifizierung eines theoretischen Konzepts - zwingend erforderlich. Durch die Kombination einer deduktiven Kategoriendefinition, basierend auf den fünf savoirs und den jeweiligen Teillernzielen interkultureller Kompetenz nach Byram (1997), mit einer induktiven Neubildung von Kategorien - also einer naturalistischen Abbildung des Materials - war es letztlich möglich, ein auf horizontaler und vertikaler Ebene modifiziertes Kategoriensystem und somit ein ausdifferenziertes Kompetenzmodell zu generieren ${ }^{3}$. Im folgenden Kapitel soll nun ein vertiefter Einblick in die konkreten Abläufe der hier erfolgten inhaltsanalytischen Datenauswertung gewährt werden. Dabei werde ich meine Konzentration auf den Prozess der Exploration und Modifizierung der horizontalen Dimension interkultureller Kompetenz richten, da die Herausarbeitung von Kompetenzstufen im Zuge einer der qualitativen Inhaltsanalyse nachgeschalteten Feinanalyse stattfand, welche sich sowohl an diskursanalytischen Ansätzen (Keller 2004) als auch an der Methode des ständigen Vergleichens (Glaser/ Strauss 2005) orientierte.

\section{Einblicke in die inhaltsanalytische Datenauswertung}

Im Zentrum jeder inhaltsanalytischen Dateninterpretation steht die Anwendung eines Kategoriensystems auf das zu untersuchende Material (Mayring 2003). Bestimmte Elemente des Materials - die sogenannten inhaltstragenden Textpassagen - werden demnach mit kurzen, vom konkreten Textausschnitt abstrahierenden Paraphrasierungen oder Zusammenfassungen versehen - den sogenannten Kategorien (Flick 2005, Gläser/Laudel 2006, Lamnek 2005, Mayring 2003).

Wesentlicher Bestandteil des Analyseprozesses ist ein an den jeweiligen Untersuchungsgegenstand angepasstes Ablaufmodell, welches jegliche $\mathrm{zu}$ beschreitende Analyseschritte und Analyseregeln enthält und zur Transparenz und Nachvollziehbarkeit der Dateninterpretation beitragen soll (Gläser/Laudel 2006, Gläser-Zikuda 2005, Mayring 2003, Wilden 2006). Die ersten beiden Stufen des allgemeinen Ablaufmodells stellen immer die Festlegung des zu analysierenden Materials sowie die Formulierung des spezifischen Forschungsinteresses einer Studie dar. Diese innerhalb jeglicher qualitativer Studien durchzuführenden Schritte habe ich weiter oben bereits angesprochen.

Die Auswahl der konkreten inhaltsanalytischen Technik stellt die dritte und nächste Stufe im allgemeinen Ablaufmodell der qualitativen Inhaltsanalyse dar. Die mit dem Ziel der Ausdifferenzierung eines theoretischen Konzepts - dem

\footnotetext{
${ }^{3}$ Die Kombination einer deduktiv und einer induktiv vorgehenden qualitativen Inhaltsanalyse stellt genau genommen eine Methodentriangulation dar, welche innerhalb einer Methode erfolgt (Flick 2005).
} 
Strukturmodell interkultureller Kompetenz von Byram (1997) - erforderliche deduktive Dateninterpretation bezeichnet Mayring (2003) als strukturierende Inhaltsanalyse. Ziel einer strukturierenden Inhaltsanalyse ist es, eine bestimmte Struktur aus dem Material mithilfe eines theoretisch hergeleiteten Kategoriensystems herauszufiltern, dessen Strukturierungsdimensionen aus der Fragestellung abgeleitet und theoretisch begründet sein müssen. ${ }^{4}$ Innerhalb jenes deduktiven Kategoriensystems dienten mir die fünf savoirs aus Byrams Kompetenzmodell (1997) - savoirs, savoir comprendre, savoir être, savoir apprendrel faire und savoir s'engager - als sogenannte Hauptkategorien. Die Hauptkategorien als gröbste Strukturierungsdimension wurden darüber hinaus anhand der die savoirs konkretisierenden Lernziele weiter aufgeschlüsselt und nahmen im Kategoriensystem die Funktion von Unterkategorien ein. Diese deduktiv definierten Unterkategorien, gleichbedeutend mit den Teilaspekten interkultureller Kompetenz innerhalb des zu generierenden Kompetenzmodells, wurden schließlich an das Material herangetragen und ggf. induktiv falsifiziert.

Deduktive Kategoriendefinition und induktive Kategorienbildung $\mathrm{zu}$ vereinen, setzt natürlich voraus, dass das zugrunde gelegte, theoretisch hergeleitete Kategoriensystem offen ist (Gläser/Laudel 2006). Für die hierfür notwendige induktive Kategorienbildung sieht Mayring (2003) die Technik der sogenannten zusammenfassenden Inhaltsanalyse vor. Dabei bekommen inhaltstragende Textpassagen neu konstruierte Unterkategorien zugewiesen, die die jeweilige Textpassage unter Beachtung des eigens festgelegten Abstraktionsniveaus zusammenfassen und in das deduktiv entworfene Kategoriensystem eingefügt werden. Im Laufe des Auswertungsprozesses werden Textstellen stets entweder unter bereits gebildete Kategorien subsumiert oder mit neuen Kategorien versehen und zugleich inhaltsähnliche Kategorien gebündelt und integriert. Somit nehmen bereits gebildete Kategorien letztlich die Rolle von deduktiv erzeugten Kategorien ein. Am Ende eines solchen kombinierten inhaltsanalytischen Vorgehens steht letztlich ein Kategoriensystem, welches die Hauptaussagen fallübergreifend in Form kurzer, abstrahierender, deduktiver wie induktiver Paraphrasen wiedergibt. Um ein möglichst homogenes System an Kategorien zu erhalten, musste das Abstraktionsniveau und der sprachliche Stil der neu hinzugewonnen Kategorien stets an den deduktiv gebildeten Kategorien ausgerichtet sein.

Die vierte und fünfte Stufe des Ablaufmodells beinhaltet die Entscheidung darüber, „welche Teile nacheinander analysiert werden [und] welche Bedingungen erfüllt sein müssen, um zu einer Kodierung zu gelangen“ (Mayring

\footnotetext{
${ }^{4}$ Genau genommen handelt es sich dabei um eine inhaltliche strukturierende Inhaltsanalyse, sprich eine Inhaltsanalyse, die eine Strukturierung eines Inhalts, eines Untersuchungsgegenstandes zum Ziel hat (Eberhardt in Vorbereitung). Mayring (2003) unterscheidet innerhalb der strukturierenden Inhaltsanalyse noch die formale, die typisierende sowie die skalierende Form der Strukturierung.
} 
2003: 43). Die Bestimmung von Analyseeinheiten ist zunächst einmal notwendig, um die Präzision der Inhaltsanalyse zu erhöhen. Die Analyseeinheiten setzen sich im Sinne Mayrings (2003) aus der Kodiereinheit, der Kontexteinheit und der Auswertungseinheit zusammen. Als Auswertungseinheiten, gleichbedeutend mit den nacheinander zu analysierenden Textteilen, wurden in der vorliegenden Studie Sätze im grammatikalischen Sinn bestimmt. Kollokationen bildeten wiederum die Kodiereinheit, sprich den kleinsten Textteil, der einer Kategorie zugerechnet werden kann. Der größte Textbestandteil, also die Kontexteinheit, bestand dagegen aus der vollständigen Antwort auf eine Fragebogenoder Interviewfrage (Eberhardt in Vorbereitung).

Die Frage, welche Voraussetzungen erfüllt sein müssen, um einem Textausschnitt einen Teilaspekt interkultureller Kompetenz zuzuweisen, ist wiederum deshalb von großer Bedeutung, weil es - so Gläser/Laudel (2006: 86) „teilweise erhebliche Differenzen [gibt] zwischen theoretischen Begriffen und den Formen, in denen sich dieser theoretische Gehalt empirisch äußert“. Demzufolge müssen bereits vor Beginn des Analyseprozesses für jede deduktive Unterkategorie sowie im Zuge der Auswertung für jede induktive Unterkategorie Indikatoren bestimmt werden, die auf semantischer Ebene Aussagen darüber machen, wann ein Materialbestandteil unter die jeweilige Kategorie fällt (Eberhardt in Vorbereitung). Die im Laufe des Auswertungsprozesses permanent zu modifizierenden Indikatoren stellen ,ein wichtiges Bindeglied zwischen den relativ abstrakten Variablendimensionen und dem empirischen Material dar" (Gläser/Laudel 2006: 202). Zum Zwecke einer beschleunigten Kodierung und einer klaren Abgrenzung verwandter Kategorien nahm ich zudem eine Unterscheidung zwischen wort- und satzsemantischen Indikatoren vor. ${ }^{5}$ Die ständige Überarbeitung der Indikatoren ist selbstredend bereits ein wesentlicher Bestandteil der konkreten Datenanalyse, welche wiederum die sechste Stufe des Ablaufmodells repräsentiert. Zum Zwecke der Intrakoder-Reliabilität (Gläser-Zikuda 2005) und der damit einhergehenden Revision von Unterkategorien interkultureller Kompetenz wurde der komplette Datensatz insgesamt drei Analysedurchläufen unterzogen. Im Sinne der kommunikativen Validierung wurden außerdem ausgewählte Textpassagen von Arbeitskollegen aus der Fremdsprachendidaktik interpretiert. Den Abschluss der Datenauswertung bildete schließlich die Interpretation der Ergebnisse, sprich des ausdifferenzierten Modells interkultureller Kompetenz im Hinblick auf die Forschungsfragen.

\footnotetext{
5 Um die Zuordnung inhaltstragender Textpassagen des Materials zu deduktiven oder induktiven Kategorien weiter zu vereinfachen, sollen darüber hinaus laut Mayring (2003) in einem weiterem Schritt konkrete Textstellen festgehalten werden, die bezüglich einer bestimmten Kategorie einen prototypischen Charakter besitzen, die sogenannten Ankerbeispiele (Eberhardt, in Vorbereitung). Ebenso bietet es sich an, Kodierregeln zu formulieren, um Abgrenzungsprobleme zwischen Kategorien zu reduzieren.
} 
Zur besseren Nachvollziehbarkeit der hier beschrittenen Stufen der inhaltsanalytischen Datenauswertung stellt die Tabelle 1 noch einmal das eben beschriebene Ablaufmodell zusammenfassend dar.

Tabelle 1: Ablaufmodell einer kombinierten induktiven und deduktiven Inhaltsanalyse

\begin{tabular}{|l|l|l|}
\hline & allgemeine Schritte des Ablaufmodells & \multicolumn{1}{|c|}{ konkrete Schritte des Ablaufmodells } \\
\hline 1 & $\begin{array}{l}\text { Formulierung des spezifischen Forschungs- } \\
\text { interesses }\end{array}$ & $\begin{array}{l}\text { Ausdifferenzierung des Strukturmodells } \\
\text { interkultureller Kompetenz von Byram (1997) }\end{array}$ \\
\hline 2 & Festlegung des zu analysierenden Materials & $\begin{array}{l}\text { interkulturelle Reflexionen von Franzö- } \\
\text { sischlernern der Jahrgangsstufe 10 gewonnen } \\
\text { durch offene Fragebögen und fokussierte } \\
\text { Interviews }\end{array}$ \\
\hline 3 & Festlegung der inhaltsanalytischen Technik & $\begin{array}{l}\text { deduktiv strukturierende und induktiv } \\
\text { zusammenfassende Inhaltsanalyse }\end{array}$ \\
\hline 5 & $\begin{array}{l}\text { Festlegung der Auswertungseinheiten } \\
\text { theoretischer Grundlage }\end{array}$ & $\begin{array}{l}\text { Auswertungseinheit: Sätze } \\
\text { Kodiereinheit: Kollokationen } \\
\text { Kontexteinheit: Fragebogen- und } \\
\text { Interviewantworten }\end{array}$ \\
\hline 6 & $\begin{array}{l}\text { strukturierende und zusammenfassende } \\
\text { inhaltsanalytische Interpretation der } \\
\text { empirischen Daten unter Berücksichtigung } \\
\text { des Erkenntnisinteresses } \\
\text { Bestimmung und Überarbeitung von } \\
\text { Indikatoren auf Grundlage der empirischen } \\
\text { Daten }\end{array}$ & $\begin{array}{l}\text { Wort- und satzsemantische Indikatoren der } \\
\text { deduktiven Unterkategorien interkultureller } \\
\text { Kompetenz }\end{array}$ \\
\hline 7 & $\begin{array}{l}\text { Revision von Unterkategorien durch } \\
\text { zusätzliche Analysedurchläufe } \\
\text { interkulturellen Reflexionen von } \\
\text { Französischlernern der Jahrgangsstufe 10 im } \\
\text { interkultureller Kompetenz } \\
\text { Wort- und satzsemantische Indikatoren } \\
\text { deduktiver und induktiver Unterkategorien } \\
\text { interkultureller Kompetenz }\end{array}$ \\
\hline auf das Erkenntnisinteresse & $\begin{array}{l}\text { Revision von Unterkategorien interkultureller } \\
\text { Kompetenz durch zusätzliche } \\
\text { Analysedurchläufe seitens des Autors und von } \\
\text { Arbeitskollegen }\end{array}$ \\
\hline Interpretation der Ergebnisse im Hinblick & $\begin{array}{l}\text { Interpretation der Ergebnisse im Hinblick auf } \\
\text { die Ausdifferenzierung eines Strukturmodells } \\
\text { interkultureller Kompetenz }\end{array}$ \\
\hline
\end{tabular}

Wie genau die inhaltsanalytische Auswertung der interkulturellen Reflexionen der Französischlerner im Hinblick auf Teilaspekte interkultureller Kompetenz, also die sechste Stufe des Ablaufmodells konkret vonstatten geht, möchte ich im Folgenden anhand der Interpretation eines ausgewählten Textausschnitts kurz darlegen. Diese Textpassage entstammt einer innerhalb des fokussierten Inter- 
views mit der Schülerin Jelena vorgenommenen Kommentierung eines Filmausschnitts, im Rahmen dessen sich in einem Pariser Fast Food-Restaurant eine sehr problembehaftete Kommunikationssituation zwischen einer französischsprachigen Angestellten und einem amerikanischen Kunden abspielt:

Also, ich habe ihn irgendwie nicht so richtig verstanden, weil er an sich, also ich kenne den Film jetzt zwar nicht, aber er hatte ja anscheinend überhaupt keine Ahnung wie/ also er hat ja gar keine Ahnung von Frankreich. Und ich meine, ich wusste schon/also ich meine, man hört ja auch was, lernt ja auch jetzt eben im Französischunterricht einfach, dass in Frankreich die Menschen Englisch oft nicht sprechen können und auch nicht wollen und das einfach dann auch nicht machen. Und er wusste das anscheinend überhaupt nicht und hat dann trotzdem immer versucht, trotzdem Englisch zu reden, weil das für ihn so selbstverständlich ist, dass jeder überall Englisch reden kann und ihn auch überall versteht. Und in Frankreich eben nicht. Ja. (...). Dass die Verkäuferin ziemlich unhöflich auch war, weil, also es ist ja einfach auch so, dass Englisch die Weltsprache ist, und eigentlich spricht es ja auch jeder und vor allem auch so in einem großen Land wie Frankreich, in so einem fortgeschrittenen/also das ist ja jetzt nicht irgendwie - weiß nicht. Und dass sie einfach für sie das so selbstverständlich war auch, dass jeder eigentlich auf Französisch sprechen müsste, der da auch hinkommt. (...). Das fand ich auch ziemlich/ ja, ist mir aufgefallen. (...). Dass sie das für selbstverständlich hielt und ihn nur angeguckt hat ,Ja, ich verstehe das aber nicht, ich will, dass du mit mir Französisch sprichst'. (...). Und das kann ja nicht jeder Tourist, der da nach Frankreich kommt, sofort Französisch sprechen.

Hingewiesen werden muss zunächst darauf, dass es sich bei dem Zitat um die Antwort auf die Frage nach schwer verständlichen oder gar unverständlichen Punkten in der gezeigten Filmszene handelt. In Entsprechung zur Komplexität der interkulturellen Reflexion wurden innerhalb der Textpassage insgesamt zehn Kodierungen mit sechs unterschiedlichen Teilaspekten interkultureller Kompetenz vorgenommen. Bevor ich näher auf die einzelnen Interpretationsschritte eingehe, soll das Zitat im Sinne der Nachvollziehbarkeit für den Leser kurz paraphrasiert werden. Aus Jelenas Kommentierung geht hervor, dass sie nicht nur das unübersehbare Problem im zu kommentierenden Dialog identifiziert, nämlich das Festhalten an der eigenen Muttersprache, darüber hinaus erklärt sie das vorliegende Missverständnis auch. Die Hauptursache für die kritische Kommunikationssituation sieht sie im Aufeinanderprallen unterschiedlicher Vorstellungen bezüglich der einzusetzenden Sprache: In eben jener Weise, wie die französische Verkäuferin davon ausgeht, dass anderssprachige Menschen im Rahmen von interkulturellen Begegnungen in Frankreich auf Französisch kommunizieren sollten, so hält es der amerikanische Kunde für selbstverständlich, dass überall auf der Welt Englisch gesprochen und verstanden wird. Des Weiteren macht Jelena darauf aufmerksam, dass die kulturspezifischen Einstellungen zum Einsatz von Fremdsprachen in interkulturellen Interaktionssituationen vor allem deshalb miteinander kollidieren, weil sich keiner der Kommunikationssteilnehmer über die Einstellungen des Gesprächspartners gegenüber der anderen Sprache beziehungsweise über den allgemeinen Stellenwert 
der anderen Sprache im Klaren zu sein scheint. Sie geht also davon aus, dass das Missverständnis nicht nur aus sprachlichen Defiziten resultiert, sondern dass dafür auch Defizite auf der affektiven und kognitiven Ebene interkultureller Kompetenz verantwortlich sind. Auf Grundlage dieser Lesart kommt in dieser Reflexion ein Teilaspekt interkultureller Kompetenz zum Vorschein, der bereits im Strukturmodell von Byram (1997) als Lernziel der Hauptkategorie savoir comprendre auftritt, und zwar die Fähigkeit, Bereiche des Missverstehens und Störungen in interkulturellen Interaktions- und Kommunikationssituationen zu identifizieren und zu erklären. Eine der zentralen Fragen, mit der man im Laufe der Datenauswertung permanent konfrontiert wird - aufgrund der längeren Textpassagen ganz besonders in der Auswertung der fokussierten Interviews - ist die nach der Größe der zu kodierenden Textpassage. Begutachtet man die vorliegende Interviewantwort, so fällt auf, dass die Fähigkeit, Bereiche des Missverstehens und Störungen in interkulturellen Interaktions- und Kommunikationssituationen zu identifizieren und zu erklären, an mehreren Stellen zutage tritt. Als satzsemantische Indikatoren können demnach folgende Sätze eingestuft werden:

- „er hatte ja anscheinend überhaupt keine Ahnung wie/ also er hat ja gar keine Ahnung von Frankreich";

- „dass in Frankreich die Menschen Englisch oft ab/ nicht sprechen können und auch nicht wollen und das einfach dann auch nicht machen. Und er wusste das anscheinend überhaupt nicht und hat dann trotzdem immer versucht trotzdem Englisch zu reden, weil das für ihn so selbstverständlich ist, dass jeder überall Englisch reden kann";

- „dass die Verkäuferin ziemlich unhöflich auch war, weil, also es ist ja einfach auch so dass Englisch die Weltsprache ist und eigentlich spricht es ja auch jeder und vor allem auch so in einem großen Land wie Frankreich";

- „und dass sie einfach für sie das so selbstverständlich war auch, dass jeder eigentlich auf Französisch sprechen müsste, der da auch hinkommt".

An einer solchen Stelle der Interpretation steht man vor einer sehr grundsätzlichen Entscheidung: entweder man weist all diesen indizierenden Phrasen jeweils die deduktive Unterkategorie Fähigkeit, Bereiche des Missverstehens und Störungen in interkulturellen Interaktions- und Kommunikationssituationen zu identifizieren und zu erklären, zu. Oder aber, und dieser Weg wurde in der hier vorgestellten Studie beschritten, man entscheidet sich dafür, eine möglichst große Textpassage zu kategorisieren und damit den Kontexteinheiten ein größeres Gewicht beizumessen. Die Beantwortung dieser Frage kann keineswegs pauschal erfolgen, sondern hat sich am jeweiligen Forschungsinteresse auszurichten. In dieser Arbeit wurde entschieden, im Zweifelsfall stets eine eher größere Textpassage mit einer Unterkategorie interkultureller Kompetenz zu 
versehen. Im vorliegenden Fall resultierte diese Entscheidung in der Kodierung der gesamten Interviewantwort. Jene Grundsatzentscheidung war mit der Annahme verbunden, dass der unmittelbare Textkontext im Hinblick auf die qualitative Unterscheidung von mit identischen Unterkategorien kodierten Äußerungen eine entscheidende Rolle spielen kann. Auf der vertikalen Dimension interkultureller Kompetenz wird also dem engen Textkontext ein differenzierender Charakter zugeschrieben (Eberhardt in Vorbereitung). Selbstredend können satzsemantische Indikatoren einbettende Äußerungen immer nur dann in eine zu kodierende Textpassage integriert und somit als Kontexteinheit begriffen werden, wenn die darin auftretenden Textelemente einerseits dem gleichen Gesprächsstimulus und somit der identischen Auswertungseinheit entspringen und andererseits durchweg dem Topos $^{6}$ der korrespondierenden Kodiereinheit sowie dem Topos der entsprechenden Unterkategorie entsprechen. Wird also innerhalb einer Schülerantwort erkennbar ein neues Topos angeschnitten, ohne thematischen Bezug auf die vorigen Reflexionen, so nehmen diese Äußerungen die Rolle eigenständiger Kontexteinheiten ein. Zusätzlich illustriert wird diese Erkenntnis anhand anderer dem vorliegenden Zitat zugewiesener Unterkategorien interkultureller Kompetenz. Schließlich führt die Analyse von Jelenas interkultureller Reflexion, wie eingangs erwähnt, noch zu weiteren Kodierungen mit deduktiven wie induktiven Teilaspekten. Eingehen möchte ich zunächst auf die folgenden Sätze:

- „ich wusste schon/ also ich meine man hört ja auch was, lernt ja auch jetzt eben im Französischunterricht einfach dass in Frankreich die Menschen Englisch oft ab/ nicht sprechen können und auch nicht wollen und das einfach dann auch nicht machen";

- „dass die Verkäuferin ziemlich unhöflich auch war, weil, also es ist ja einfach auch so, dass Englisch die Weltsprache ist und eigentlich spricht es ja auch jeder und vor allem auch so in einem großen Land wie Frankreich“.

Trotz starker Verallgemeinerungen kommt nicht nur aufgrund des expliziten Hinweises ,ich wusste schon“ in den Äußerungen ein subjektives Wissen ${ }^{7}$ zum Vorschein, welches sich auf Kommunikationsprozesse und Einstellungen von Menschen in Frankreich richtet. In Anbetracht der Tatsache, dass im Gegensatz zur vorigen Unterkategorie hier der enge Textkontext im Hinblick auf die Wissensqualität als unerheblich erachtet wird, wurden im vorliegenden Fall ausschließlich die einzelnen Sätze kodiert: die erste Äußerung wird einerseits als

\footnotetext{
${ }^{6}$ Das Topos (Eberhardt in Vorbereitung) oder auch Thema einer kodierten Textpassage basiert stets auf den darin enthaltenen Textelementen auf wort- und satzsemantischer Ebene.

${ }^{7}$ Kallenbach (1996) versteht unter subjektivem Wissen das aus individuellen Erfahrungen gewonnene Alltagswissen. Mit diesem Begriff wird der idiosynkratische Charakter von Wissen zum Ausdruck gebracht.
} 
Wissen über Interaktions- und Kommunikationsprozesse und -eigenschaften anderer Kommunikationsgemeinschaften sowie als Wissen über Denkweisen, Einstellungen, Verhaltensweisen und Bräuche anderer Kommunikationsgemeinschaften verstanden und andererseits als Bewusstsein über die kulturelle Bedingtheit des menschlichen Denkens, Fühlens und Handelns. Die zweite Phrase wiederum kann der Unterkategorie Wissen über die mit interkulturellen Begegnungen verbundenen Anforderungen und Schwierigkeiten sowie deren Ursachen zugerechnet werden.

Neben Unterkategorien der Hauptkategorien savoir comprendre und savoirs zeichnet sich in der hier diskutierten Reflexion auch ein Teilaspekt ab, welcher im Überschneidungsbereich zwischen kognitiver und affektiver Ebene interkultureller Kompetenz anzusiedeln ist. Im ersten Teil ihrer Antwort befasst Jelena sich mit dem Verhalten des amerikanischen Kunden. Zunächst stellt die Französischlernerin Vermutungen auf, welche die Unwissenheit des Amerikaners zum Inhalt haben (,dass in Frankreich die Menschen Englisch oft nicht sprechen können und auch nicht wollen und das einfach dann auch nicht machen. Und er wusste das anscheinend überhaupt nicht") sowie seine Einstellung zur englischen Sprache (,,weil das für ihn so selbstverständlich ist, dass jeder überall Englisch reden kann und ihn auch überall versteht"). Anschließend unternimmt die Schülerin den Versuch, sich in die französischsprachige Verkäuferin hineinzuversetzen und deren Einstellung zum Gebrauch der französischen Sprache zu ergründen („Und dass sie einfach für sie das so selbstverständlich war auch, dass jeder eigentlich auf Französisch sprechen müsste, der da auch hinkommt"). Diese affektiv-kognitiven Operationen spiegeln die Bereitschaft zur Perspektivendarstellung und Perspektivenübernahme im Rahmen kulturell aufgeladener Situationen wider (Bechtel 2003), einen induktiven Teilaspekt der Hauptkategorie savoir être. Im Gegensatz zu den eben behandelten Wissenskategorien muss hier das gesamte Zitat einer Kodierung unterzogen werden, da der enge Textkontext thematisch konform mit den satzsemantischen Indikatoren geht.

Nachdem mit der Diskussion des obigen Zitats dem Leser ein kleiner Einblick in den inhaltsanalytischen Interpretationsprozess gewährt werden sollte, möchte ich im letzten Kapitel meines Beitrags vor dem Hintergrund der Ergebnisse meines Forschungsprojekts auf den Ertrag der qualitativen Inhaltsanalyse eingehen.

\section{$4 \quad$ Ertrag der qualitativen Inhaltsanalyse}

In Entsprechung zum Forschungsinteresse des Projekts stand am Ende des inhaltsanalytischen Auswertungsprozesses ein empirisch ausdifferenziertes Kompetenzmodell, welches sich aus insgesamt 35 Teilaspekten interkultureller Kom- 
petenz zusammensetzt. Trotz der erfolgreichen Verfeinerung des Strukturmodells von Byram (1997) muss der Ertrag der qualitativen Inhaltsanalyse im Hinblick auf die Ausdifferenzierung der horizontalen Dimension interkultureller Kompetenz kritisch diskutiert werden.

Ein Kritikpunkt, welcher innerhalb der empirischen Sozialforschung häufig im Zusammenhang mit der qualitativen Inhaltsanalyse geäußert wird, betrifft deren Tiefgründigkeit (Eberhardt in Vorbereitung). Als exemplarisch hierfür kann folgende Aussage von Flick (2005: 283) betrachtet werden:

Gerade durch die Schematisierung des Vorgehens und die Art der Ausformulierung der einzelnen Schritte ist der Ansatz stark vom Ideal standardisierter Methodik geprägt. Die schnelle Kategorisierung mit von außen herangetragenen, theoretisch begründeten Kategorien lenkt möglicherweise den Blick eher auf den Inhalt des Textes, als dass sie den Text und seine (Un-) Tiefen auszuloten erleichtert. Interpretation des Textes im Sinne der meisten anderen Auswertungsverfahren wird eher schematisch durch die explikative Inhaltsanalyse realisiert.

Der hier geäußerten Kritik möchte ich jedoch entschieden entgegentreten. Denn meines Erachtens übersehen die Vertreter dieser Position, dass die indikatorbasierte Interpretation der qualitativen Inhaltsanalyse jedem Forscher an vielen Stellen des Ablaufmodells einen großen Spielraum lässt. Insbesondere die stets individuell zu treffenden Entscheidungen über die Größe der Kodier- und Kontexteinheiten sind dafür verantwortlich, wie weit sich die Dateninterpretation von der Denotation einzelner Wörter und der Textoberfläche entfernt und auch konnotative Wortbedeutungen und pragmalinguistische Aspekte in der Interpretation verbaler Aussagen miteinbezieht, inwiefern sie einzelne Sätze und Textpassagen nicht isoliert, sondern in ihrem Kontext betrachtet, und damit auch die Rekonstruktion latenter Sinneinheiten ermöglicht (Eberhardt in Vorbereitung). Konsequenterweise weisen Gläser/Laudel (2006: 195) auch darauf hin, dass nicht nur die induktive, sondern auch die deduktive Inhaltsanalyse vielfältige Interpretationen impliziert: „Um festzustellen, ob relevante Informationen in einem Text enthalten sind, müssen Sie ihn interpretieren. Auch die Zuordnung zu einer Kategorie und die verbale Beschreibung des Informationsinhaltes beruhen jeweils auf Interpretationen des Textes.“

Die im vorliegenden Artikel aufgeführten zahlreichen Vorteile einer inhaltsanalytischen Dateninterpretation - in erster Linie die Möglichkeit einer Kombination von deduktiver und induktiver Auswertung, die Bearbeitung großer Datenmengen sowie ein transparentes, regelgeleitetes Vorgehen - haben demzufolge nicht nur dazu beigetragen, ein empirisch ausdifferenziertes Modell interkultureller Kompetenz für den standardorientierten Französischunterricht der Sekundarstufe I zu generieren, es sollte darüber hinaus auch deutlich geworden sein, dass ihre individuelle projektspezifische Auslegung vielerlei Interpretationsspielräume bietet. Aufgrund dessen muss die qualitative Inhaltsanalyse als ein Interpretationsverfahren betrachtet werden, dem trotz oder gerade wegen 
seiner aus dem quantitativen hypothesenüberprüfenden Paradigma herrührenden Einflüsse eine tragende Rolle im Kontext der aktuellen empirischen Bildungsforschung zukommt.

\section{Literatur}

Aguado, Karin / Schramm, Karen / Vollmer, Helmut Johannes (Hrsg.) (2010). Fremdsprachliches Handeln beobachten, messen, evaluieren. Frankfurt a. M.: Peter Lang.

Bausch, Karl-Richard / Burwitz-Melzer, Eva / Königs, Frank G. / Krumm, Hans-Jürgen (Hrsg.) (2005). Bildungsstandards für den Fremdsprachenunterricht auf dem Prüfstand. Arbeitspapiere der 25. Frühjahrskonferenz zur Erforschung des Fremdsprachenunterrichts. Tübingen: Narr.

Bechtel, Mark (2003). Interkulturelles Lernen beim Sprachenlernen im Tandem. Eine diskursanalytische Untersuchung. Tübingen: Narr.

Bennett, Milton J. (1986). A developmental approach to training for intercultural sensitivity. International Journal of Intercultural Relations 10: 179-196.

Bolten, Jürgen (2007). Was heißt "Interkulturelle Kompetenz"? Perspektiven für die internationale Personalentwicklung. In: Berninghausen, Jutta / Kuenzer, Vera (Hrsg.): Wirtschaft als interkulturelle Herausforderung. Business across Cultures. Frankfurt: IKO. 21-42.

Bredella, Lothar / Christ, Herbert (Hrsg.) (2007). Fremdverstehen und interkulturelle Kompetenz. Tübingen: Narr.

Byram, Michael (1997). Teaching and assessing intercultural communicative competence. Clevedon: Multilingual Matters.

Chen, Guo-Ming / Starosta, William J. (2005). Foundations of intercultural communication. New York: University Press of America.

Eberhardt, Jan-Oliver (2008). Interkulturelle Kompetenz bei Französischlernern. Eine explorative Studie mit Schülern der Jahrgangsstufe 10. Zeitschrift für Fremdsprachenforschung 19/2: 273-296.

Eberhardt, Jan-Oliver (2009). "Flaggen, Baguettes, auch wenn's komisch klingt, das Aussehen der Leute erinnert an Frankreich". Von den Herausforderungen, interkulturelle Kompetenz im Fremdsprachenunterricht zu evaluieren. In: Hu, Adelheid / Byram, Michael (Hrsg.): Interkulturelle Kompetenz und fremdsprachliches Lernen. Modelle, Empirie, Evaluation. Intercultural competence and foreign language learning. Models, empiricism, assessment. Tübingen: Narr. 253-272.

Eberhardt, Jan-Oliver (2010). Interkulturelle Kompetenz als Bildungsstandard im Fremdsprachenunterricht: zwischen Anspruch und Wirklichkeit. In: Altmayer, Claus / Mehlhorn, Grit / Neveling, Christiane / Schlüter, Norbert (Hrsg.): Grenzen überschreiten: fachlichsprachlich - kulturell. Dokumentation zum 23. Kongress für Fremdsprachendidaktik. Baltmannsweiler: Schneider Hohengehren.

Eberhardt, Jan-Oliver (in Vorbereitung). Interkulturelle Kompetenz im outputorientierten Fremdsprachenunterricht. Empirische Ausdifferenzierung eines Kompetenzstrukturmodells. Hamburg: Universität Hamburg. 
Erpenbeck, John / Rosenstiel, Lutz von (2003). Einführung. In: Erpenbeck, John / Rosenstiel, Lutz von (Hrsg.): Handbuch Kompetenzmessung. Erkennen, verstehen und bewerten von Kompetenzen in der betrieblichen, pädagogischen und psychologischen Praxis. Stuttgart: Schäffer-Poeschel. IX-XL.

Flick, Uwe (2005). Qualitative Sozialforschung. Eine Einführung. Reinbek: Rowohlt.

Gläser, Jochen / Laudel, Grit (2006). Experteninterviews und qualitative Inhaltsanalyse als Instrumente rekonstruierender Untersuchungen. Wiesbaden: VS Verlag.

Gläser-Zikuda, Michaela (2005). Qualitative Inhaltsanalyse in der Lernstrategie- und Lernemotionsforschung. In: Mayring, Philipp / Gläser-Zikuda, Michaela: Die Praxis der Qualitativen Inhaltsanalyse.Weinheim: Beltz UTB. 63-83.

Glaser, Barney G. / Strauss, Anselm L. (2005). Grounded Theory. Strategien qualitativer Forschung. Bern: Huber.

Goethe-Institut / Inter Nationes / Ständige Konferenz der Kultusminister der Länder in der Bundesrepublik Deutschland (KMK) / Schweizerische Konferenz der Kantonalen Erziehungsdirektoren (EDK) / Österreichisches Bundesministerium für Bildung, Wissenschaft und Kultur (BMBWK) (Hrsg.) (2001). Gemeinsamer europäischer Referenzrahmen für Sprachen: lernen, lehren, beurteilen. Berlin: Langenscheidt.

Grünewald, Andreas / Küster, Lutz (Hrsg.) (2009). Fachdidaktik Spanisch. Tradition. Innovation. Praxis. Stuttgart: Klett.

$\mathrm{Hu}$, Adelheid (2008). Interkulturelle Kompetenz. Ansätze zur Dimensionierung und Evaluation einer Schlüsselkompetenz fremdsprachlichen Lernens. In: Frederking, Volker (Hrsg.): Schwer messbare Kompetenzen. Herausforderungen für die empirische Fachdidaktik. Baltmannsweiler: Schneider Hohengehren. 11-35.

$\mathrm{Hu}$, Adelheid / Byram, Michael (Hrsg.) (2009). Interkulturelle Kompetenz und fremdsprachliches Lernen. Modelle, Empirie, Evaluation. Intercultural competence and foreign language learning. Models, empiricism, assessment. Tübingen: Narr.

Kallenbach, Christiane (1996). Subjektive Theorien. Was Schüler und Schülerinnen über Fremdsprachenlernen denken. Tübingen: Narr.

Keller, Reiner (2004). Diskursforschung. Eine Einführung für SozialwissenschaftlerInnen.Wiesbaden: VS Verlag.

Klieme, Eckhard (2003). Zur Entwicklung nationaler Bildungsstandards. Eine Expertise. Bonn: BMBF.

Klieme, Eckard / Leutner, Detlev (2006). Kompetenzmodelle zur Erfassung individueller Lernergebnisse und zur Bilanzierung von Bildungsprozessen. Beschreibung eines neu eingerichteten Schwerpunktprogramms der DFG. Zeitschrift für Pädagogik 52/6: 876-903.

Köller, Olaf (2008): Bildungsstandards - Verfahren und Kriterien bei der Entwicklung von Messinstrumenten. Zeitschrift für Pädagogik 54/2: 163-173.

Lamnek, Siegfried (2005). Qualitative Sozialforschung. Lehrbuch. Weinheim: Beltz PVU.

Mayring, Philipp (2003). Qualitative Inhaltsanalyse. Grundlagen und Techniken. Weinheim: Beltz UTB. 
Rehm, Markus (2006). Allgemeine naturwissenschaftliche Bildung - Entwicklung eines vom Begriff "Verstehen" ausgehenden Kompetenzmodells. Zeitschrift für Didaktik der Naturwissenschaften 12: 23-44.

Sercu, Lies (2004). Assessing intercultural competence: a framework for systematic test development in foreign language education and beyond. Intercultural Education 15/1: 7389.

Ständige Konferenz der Kultusminister der Länder in der Bundesrepublik Deutschland (KMK) (2003). Beschlüsse der Kultusministerkonferenz. Bildungsstandards für die erste Fremdsprache (Englisch/Französisch) für den Mittleren Schulabschluss. Neuwied: Luchterhand.

Tesch, Bernd / Leupold, Eynar / Köller, Olaf (Hrsg.) (2008). Bildungsstandards Französisch: konkret. Grundlagen, Aufgabenbeispiele und Unterrichtsanregungen. Berlin: Cornelsen Scriptor.

Thomas, Alexander (2003). Kultur und Kulturstandards. In: Thomas, Alexander / Kinast, Eva-Ulrike / Schroll-Machl Sylvia (Hrsg.): Handbuch interkulturelle Kommunikation und Kooperation. Band 1: Grundlagen und Praxisfelder. Göttingen: Vandenhoeck \& Ruprecht. 19-31.

Wilden, Eva (2006). Die Qualitative Inhaltsanalyse in einer Längsschnittstudie: Zum Selbstbild und Fremdbild in einem interkulturellen Onlineaustausch. Beiträge zur Qualitativen Inhaltsanalyse 13: 1-14.

Zydatiß, Wolfgang (2006). Stehen wir vor einem meltdown der Persönlichkeitsbildung im schulischen Fremdsprachenunterricht? - Vermutlich ja, aber gerade deshalb sollten empirisch erprobte, integrierte Lern- und Überprüfungsaufgaben für diese Bereich entwickelt werden!. In: Bausch, Karl-Richard / Burwitz-Melzer, Eva / Königs, Frank G. / Krumm, Hans-Jürgen (Hrsg.): Aufgabenorientierung als Aufgabe. Arbeitspapiere der 26. Frühjahrskonferenz zur Erforschung des Fremdsprachenunterrichts. Tübingen: Narr. 256-264. 


\title{
Die qualitative Inhaltsanalyse als Mittel zur empirischen Konkretisierung fremdsprachendidaktischer Paradigmata am Beispiel der inter- bzw. transkulturellen Kompetenzen in fremdsprachlichen Begegnungsprojekten
}

\author{
Michael Rogge
}

\begin{abstract}
The method of Qualitative Content Analysis (Mayring 2008) opens up new ways of systematic, rule-guided analysis of larger text corpora along the lines of a theoretically constructed system of analytic criteria and helps to identify both manifest and latent text content. Therefore, the method seems well suited to analyse student documentations of foreign language encounter projects and develop a system of categories that contribute to the ongoing discussion about intercultural communicative competences and/or intercultural learning in the context of institutionalised foreign language learning. However, Mayring himself claims that the method has to be adapted to domain-specific research needs. The following article aims at discussing some modifications of Mayring's research approach based on specific research needs and related methodological questions.
\end{abstract}

\section{Interkulturelle Kommunikative Kompetenz - ein noch unzureichend geklärtes Paradigma der Fremdsprachen- didaktik}

Interkulturelle kommunikative Kompetenzen gehören zu den zentralen Schlüsselqualifikationen schulischer Bildung und bilden eines der Hauptziele des schulischen Fremdsprachenunterrichts (vgl. Bredella/Christ 1995, Council of Europe 2001). Sie werden allgemein als die Fähigkeit verstanden, „mit Menschen zu kommunizieren und zu interagieren, die eine andere Sprache sprechen und in einem anderen kulturellen Kontext leben" (Freitag 2010: 121). Der dem Konstrukt zugrunde liegende interkulturelle Kompetenzbegriff hat dabei im schulischen Fremdsprachenunterricht das Paradigma der kommunikativen Kompetenzen abgelöst und definiert damit als Ziel des Fremdsprachenlernens nicht länger einen (für Fremdsprachenlerner nahezu) unerreichbaren Standard des native speaker, sondern des intercultural speaker, d. h. das Verstehen fremder Kulturen, das Anwenden kulturell bedingter und der sozialen Situation angemessener sprachlicher Formen rückt in den Vordergrund (Byram 1997: 32, Kramsch 1998: 27). Darüber hinaus erhalten kulturspezifische Regeln, Konventionen, Werte und Einstellungen besonderes Gewicht, damit Schülerinnen und Schüler lernen, wie man ,in bestimmten Situationen das Richtige sagt, keine Tabus verletzt und den Anderen angemessen versteht" (Bredella 1999: 91). Damit verknüpft sind individuelle Dispositionen wie die Fähigkeit zum Perspektivwechsel und zur Empathiefähigkeit, zur Überwindung eigener (ethnozentrischer) Perspektiven zugunsten einer ethnorelativen Haltung und die Neugier 
und Offenheit gegenüber Fremdem im Fremdsprachenunterricht. Doch in Zeiten von Globalisierung und zunehmender Heterogenisierung von Gesellschaften ist der Begriff des interkulturellen Lernens zunehmend in die Kritik geraten. Mit dem Begriff des transkulturellen Lernens hat Welsch $(1992,1994)$ daher einen Gegenentwurf zum Konzept der interkulturellen Kompetenz vorgelegt, der nicht von einer binären Perspektive ausgeht, d. h. das Verstehen zwischen Eigenkultur und Zielkultur in den Vordergrund stellt, sondern das Hin- und Herwechseln zwischen verschiedensten kulturellen Perspektiven als zentrales Moment kulturellen Verstehens ansieht. Der transkulturelle Ansatz Welschs hat dabei nicht nur in der Kultur- und Literaturdidaktik (vgl. Antor 2006, Freitag-Hild 2009) und der Fremdsprachendidaktik (vgl. Delanoy 2006, Fäcke 2006) zu neuen Einsichten geführt, sondern in dem neuen Kerncurriculum in Hessen sogar den Begriff der interkulturellen Kompetenz gänzlich verdrängt. Dort heißt es:

Transkulturelle Kompetenz beinhaltet die Fähigkeit und Bereitschaft, unterschiedliche kulturelle Perspektiven wahrzunehmen, sie zu respektieren und von ihnen zu lernen. Dazu gehört die Einsicht, dass das Denken, Handeln und Verhalten zwar immer von der eigenen Kultur geprägt ist, jedoch auch verändert werden kann. Transkulturelle Kompetenz umfasst darüber hinaus das Wissen über die eigene Kultur und andere Kulturen. Sie ermöglicht einen differenzierten Blick auf ökologische und ökonomische Aspekte in einer globalisierten Welt. Eine selbstbewusste, offene und wertschätzende Haltung gegenüber anderen Kulturen zeigt sich im Denken, Fühlen und Handeln. (Kerncurriculum Hessen 2011:16)

Es stellt sich daher die Frage, ob und inwieweit der Begriff der transkulturellen Kompetenz insbesondere für fremdsprachliche Begegnungsprojekte, in denen es darum gehen sollte, unterschiedliche kulturelle Perspektiven wahrzunehmen und in der Interaktion mit Anderen zu einer differenzierteren kulturellen Wahrnehmung zu gelangen, von besonderer Bedeutung sein könnte bzw. das bisherige Modell interkultureller kommunikativer Kompetenz ergänzen, gegebenenfalls sogar ersetzen könnte. Um Welschs Ansatz und die wesentlichen Unterschiede zwischen einem inter- und einem transkulturellen Blick auf kulturelle Begegnungen zu verstehen, lohnt ein Blick auf die gängigen Modelle, die kulturellem Verstehen zugrunde liegen, um abschließend abzuwägen, welches Modell sich für den Kontext des Forschungsvorhabens in besonderer Weise eignet.

\subsection{Das traditionelle Konzept von Kultur als in sich abgeschlossene Entitäten}

Dem fremdsprachlichen Unterricht liegt immer ein bestimmtes kulturelles Verständnis zugrunde, das auf verschiedene Weise realisiert werden kann: Als Realien- bzw. Landeskunde oder als cultural studies hat die Beschäftigung mit der Zielkultur einen festen Platz im Fremdsprachenunterricht. 
Ausgehend von Herders Kugelmodell, das Kulturen als abgeschlossene, sich gegenseitig abstoßende Kugeln auffasste, wurden Kulturen als homogene, in sich abgeschlossene Einheiten aufgefasst, die nicht im direkten Kontakt oder Austausch mit anderen kulturellen Einheiten stehen. Herder formulierte dazu:

Jede Nation hat ihren Mittelpunkt der Glückseligkeit wie jede Kugel ihren Schwerpunkt [...] Alles was mit meiner Kultur noch gleichartig ist, was in sie assimiliert werden kann, beneide ich, strebe an, mache mir zu eigen; darüber hinaus hat mich die gütige Natur mit Fühllosigkeit, Kälte und Blindheit bewaffnet; sie kann gar Verachtung und Ekel werden. (Herder 1984: 617f.)

Vor diesem Hintergrund eines auf Homogenität und Abgegrenztheit definierten Kulturbegriffes sind interkulturelle Erfahrungen in Austausch- und Begegnungsprojekten nur schwer möglich und fokussieren zumeist auf kulturelle Unterschiede, die in der Begegnung als trennend empfunden werden, auch wenn neuere kulturdidaktische Ansätze (vgl. Altmayer 2004) die sich durch Globalisierung und Hybridisierung von Kultur ergebenden Konsequenzen für den Fremdsprachenunterricht durchaus reflektieren.

\subsection{Byrams Modell interkultureller kommunikativer Kompetenz als Modell eines interkulturellen Dialogs}

Das von Byram (1997) entwickelte Modell interkultureller Kompetenz (Abb.1) beschreibt individuelle Kompetenzen in den Domänen Wissen, Fähigkeiten und Einstellungen, wobei die Vermittlung kulturellen Wissens (knowledge) als Wissen über soziale Gruppen und über Interaktionsprozesse zwischen diesen angesehen wird und nicht nur als reines Faktenwissen über die Zielkultur verstanden wird. Daher werden kulturelle Informationen stets in Beziehung zur eigenen Kultur gesetzt (relationales Wissen, vgl. ebd.: 51). Darüber hinaus bedarf es aber auch der erforderlichen Einstellungen und Haltungen (attitudes) wie z. B. Offenheit und Neugier gegenüber dem Anderen oder des Aushaltens von Unterschieden und der Relativierung eigener Perspektiven. Über spezifische Fähigkeiten des kulturellen Verstehens und Einordnens (skills of interpreting and relating) sowie (im Sinne ethnographischer Ansätze) der Fähigkeiten zur Interaktion und des Entdeckens neuer kultureller Aspekte (skills of discovery and/or interaction) soll den Schülerinnen und Schülern (insbesondere durch eine möglichst authentische Beschäftigung mit der fremdsprachlichen Welt) dabei die Zielkultur bzw. der Gegenstand des Fremdsprachenunterrichts näher gebracht werden (vgl. Byram/ Risager 1999: 123f.). Hinzu kommt in Byrams Modell, dass die Zielsetzung in der Förderung eines kritischen kulturellen Bewusstseins (critical cultural awareness) und der politischen Bildung liegt, d. h. es ist die Entwicklung von komplexen Handlungsdispositionen angestrebt, die es den Schülerinnen und Schülern ermöglichen, ihre eigene und die fremde Kultur kritisch $\mathrm{zu}$ sehen und $\mathrm{zu}$ hinterfragen. Daher ist der Vergleich zwischen den Kulturen ein wesentlicher 
Schritt, um Unterschiede, aber auch Gemeinsamkeiten zu entdecken und mit dem Begegnungspartner auszuhandeln sowie im Sinne eines intercultural speaker zwischen der eigenen Perspektive und der Sichtweise des Anderen zu vermitteln.

\begin{tabular}{|c|c|c|}
\hline & $\begin{array}{l}\text { Skills } \\
\text { Interpret and relate } \\
\text { (savoir comprendre) }\end{array}$ & \\
\hline \multirow[t]{2}{*}{$\begin{array}{l}\text { Knowledge } \\
\text { of self and other; } \\
\text { of interaction: } \\
\text { individual and } \\
\text { societal (savoirs) }\end{array}$} & $\begin{array}{l}\text { Education } \\
\text { Political education / } \\
\text { critical cultural } \\
\text { awareness } \\
\text { (savoir s'engager) }\end{array}$ & $\begin{array}{l}\text { Attitudes } \\
\text { Relativising self } \\
\text { Valuing other } \\
\text { (savoir être) }\end{array}$ \\
\hline & $\begin{array}{l}\text { Skills } \\
\text { Discover and / or } \\
\text { interact } \\
\text { (savioir apprendre / } \\
\text { faire) }\end{array}$ & \\
\hline
\end{tabular}

Abbildung 1: Byrams Modell der interkulturellen kommunikativen Kompetenz (Byram 1997: 34)

Die Kritik an Byrams Modell bezieht sich zum Einen auf die Darstellung von interkulturellen Teilkompetenzen als skills, d. h. als erlernbare Fähigkeiten, wie Belz (2003) betont : "[...] it carries with it the negative connotation that these components might be 'learnt by a simple technology and transferred unproblematically' from one context to another" (Belz 2003: 5). Tatsächlich handelt es sich im Sinne Weinerts (2001) um sehr komplexe Kompetenzbereiche. Müller-Hartmann/Schocker-von Ditfurth (2008) sprechen in diesem Zusammenhang von „hochkomplexe(n) menschlichen(n) Aktivitäten. Wir gehen deshalb davon aus, dass es für die Entwicklung interkultureller Kompetenzen eines vielschichtigen Lernszenarios bedarf, das im Idealfall die direkte Erfahrung einer interkulturellen Begegnung integriert" (Müller-Hartmann/Schocker-von Ditfurth 2008: 125).

Aus dieser Sicht ergibt sich quasi eine Notwendigkeit, interkulturelle Kompetenzen nicht nur innerhalb des Klassenzimmers zu entwickeln, sondern gerade die von Byram als skills definierten Teilkompetenzen in der praktischen Anwendung in Austausch- und Begegungsprojekten zu erproben und weiterzuentwickeln. Sie können aber nur bedingt im unterrichtlichen Kontext wie strategische Kompetenzen entwickelt und geübt werden.

Ein zweiter Kritikpunkt an Byrams Modell betrifft den streng interkulturellen Zugriff auf die individuellen Lernprozesse der Schülerinnen und Schüler, der sich aus der durchweg homogenen Sicht auf die fremde Kultur ergibt. Ein streng homogener Blick auf Eigen- und Fremdkultur kann den Eindruck vermitteln „[...] als bräuchte man nur über Gewohnheiten, Werte und Einstellungen der fremden Kultur Bescheid zu wissen, um die Menschen dieser Kultur zu ver- 
stehen“ (Bredella 1999: 91). Bredella betont, dass das Verhalten der Menschen nicht direkt aus den Regeln und Werten ihrer Kultur ableitbar sei:

Versteht man Kultur als System von Gewohnheiten, Einstellungen und Werten, dann übersieht man, dass Menschen ihre Kultur durch Handlungen und Verhandlungen aufrechterhalten. Kultur determiniert nicht das Handeln der Menschen, sondern schafft für sie nur einen Rahmen, in dem sie handeln. (Bredella 1999: 91)

Gerade in der heutigen Zeit ist dieser kulturelle Rahmen aber keinesfalls mehr streng homogen strukturiert, sondern zeichnet sich durch eine Pluralität kultureller Muster, durch Heterogenität und Hybridität aus, die durch ein Modell, das weitgehend auf Wahrnehmung von Differenzen bzw. Gemeinsamkeiten zwischen Eigenem und Fremden ausgerichtet ist, nur unzureichend erfasst werden kann.

\subsection{Welschs Modell der transkulturellen Durchdringung und Verflechtung}

Welsch beschreibt Kulturen als hybride Konstrukte, wobei diese Hybridität nicht nur auf der gesellschaftlichen Makroebene, sondern vor allem auf der individuellen Mikroebene evident wird: „Die meisten unter uns sind in ihrer kulturellen Formation durch mehrere kulturelle Herkünfte und Verbindungen bestimmt. Wir sind kulturelle Mischlinge. Die kulturelle Identität der heutigen Individuen ist eine patchwork-Identität [...] Heutige Menschen werden zunehmend in sich transkulturell" (Welsch 2010: 43). Diese individuelle Hybridität erleichtert es aber dem Einzelnen zugleich, Gemeinsamkeiten bzw. Schnittmengen mit anderen Individuen - ungeachtet ihrer kulturellen Herkunft - zu finden, die aus dem interkulturellen Diskurs bekannte Unterscheidung zwischen ,Eigenem“ und ,Fremdem‘ löst sich entsprechend auf (ebd.: 45). Während Welschs Konzeption einem gewissen Absolutheitsanspruch folgt, betonen andere Vertreter, dass das Interkulturalitätsmodell durchaus seine Berechtigung habe. Schulze-Engler (2006: 47) sieht eine „Gleichzeitigkeit des ,Inter-' und des Transkulturellen" und stellt heraus, dass in Zeiten von Rassismus, Fremdenfeindlichkeit und Ethnozentrismus die von Welsch (1994: 159) propagierte Aufhebung der Kulturen keinesfalls erreicht sei und dem Konzept der Interkulturalität die gesellschaftliche und pädagogische Aufgabe zukomme, durch Förderung des interkulturellen Dialogs zu einem Abbau von Stereotypen beizutragen (Schulze-Engler 2006: 45).

Dagegen kritisiert Bredella (2010) das Konzept der Transkulturalität und betont, dass das Ziel des Fremdsprachenunterrichts nicht in der „Abschaffung, sondern der Anerkennung kultureller Unterschiede bestehen müsse" (Bredella 2010: 21). Er betont mit Bezug auf Vasilache (2003) eine Komplexität des Begriffs der Interkulturalität, die sich aus der Wechselwirkung zwischen den 
Momenten der Hybridität, der Historizität, der Sinnhaftigkeit von Kultur und Vergleichbarkeit ergibt und die gerade im Fremdsprachenunterricht einen weitaus komplexeren Umgang mit Kulturen ermöglicht, als dies ein herkömmlicher landeskundlich orientierter Fremdsprachenunterricht oder auch ein streng transkultureller (im Sinne einer Ausblendung kultureller Grenzen und Unterschiede) orientierter Unterricht zu leisten imstande wäre. Für die Schülerinnen und Schüler stellt gerade die Auseinandersetzung mit dem ,Fremden' und dem ,Eigenen' im Sinne einer Selbstvergewisserung einen wichtigen Schritt zur Entwicklung von inter- und transkulturellen Kompetenzen dar.

Grundsätzlich lassen sich trans- und interkulturelle Kompetenzen bei Schülerinnen und Schülern sicherlich nur schwer trennen: Sie erfahren die transkulturelle Welt oftmals ohne eindeutige Zuweisungen von Eigenem und Fremden, erleben eine Hybridität von Alltagskultur, ein gleichberechtigtes Nebeneinander von türkischem Döner Kebab, indischer Bollywood-Folklore, asiatischem Kampfsport und der scheinbar globalen Begeisterung für amerikanische Pop-Kultur. Doch zugleich betonen sie als Jugendliche in der Adoleszensphase regionale und partikulare Unterschiede, wie z. B. die Herkunft aus bestimmten Stadtteilen innerhalb einer Großstadt oder die Verbundenheit zu einem bestimmten Fußballklub und bestehen auf territorialen wie kulturellen Grenzziehungen (vgl. Latour 2007: 59).

Insbesondere für Schülerinnen und Schüler mit Migrationshintergrund werden solche Grenzziehungen keinesfalls obsolet, sondern bekommen besonderes Gewicht (vgl. Tajfel 1982). Von einer Stereotypisierung durch Bezugnahme auf Kulturen, wie Eckert/Wendt (2003: 12) betonen, ist hier kaum zu sprechen, sondern vielmehr von einer bewussten Verstehens- und Interpretationsleistung der jeweiligen Individuen, für die eine Ausrichtung an kulturellen Symbolsystemen eine wichtige Orientierungshilfe darstellt (vgl. Bruner 1990, Straub 1999).

In diesem Sinne begründet auch Hallet (2011) sein Konzept des Fremdsprachenunterrichtes als hybridem Raum, wenn er vorschlägt,

[...] sich den Englischunterricht als einen transkulturellen (hybriden) Diskursraum vorzustellen, in dem diskursive Elemente, Denkweisen und kulturelle Prägungen aus verschiedenen kulturellen Diskurssphären in ein interplay, in ein „Spiel der Texte und Kulturen" eintreten und zu neuen Orientierungen der Lernenden beitragen oder führen. (Hallet 2011: 100)

Auch die auf diese Weise im Fremdsprachenunterricht neu entstehenden Denkweisen, Vorstellungen und eventuell sogar Verhaltensweisen kann man transkulturell nennen, weil sie sich nicht einem bestimmten (oder ihrem ursprünglichen) kulturellen Kontext zuordnen lassen, sondern sich gerade solchen kulturellen (oder nationalkulturellen) Zuordnungen entziehen (ebd., vgl. Hallet 2002: 42).

Hallet unterscheidet in diesem Zusammenhang zwischen verschiedenen Diskurssphären (Diskurse der Lebenswelt der Lernenden; Diskurse der anglophone 
Kulturen und Gesellschaften und transkulturelle, mehrkulturelle bzw. globale Diskurse), die im Fremdsprachenunterricht durch entsprechende Auswahl von Themen und Materialien aufeinander bezogen werden können:

Das generelle Ziel der Diskursfähigkeit erhält auf diese Weise eine mehrsprachige und mehrkulturelle Dimension, die sich sowohl als interkulturelle Differenz als auch als transkulturelle Aufladung oder Bereicherung darstellen kann. Der Idealfall kulturellen Lernens ist dann das transkulturelle Lernen, das sich als Ergebnis interkulturellen Verstehens und Aushandelns verstehen lässt: Die Lernenden nehmen kulturelle Elemente (Vorstellungen, Perspektiven, Rede- und Ausdrucksweisen, Stile) aus anglophonen Diskursen auf, transformieren und adaptieren sie und integrieren sie in ihr eigenenes kulturelles Repertoire. Dies ist ein kultureller und kommunikativer Transferprozess, der sich im Zeitalter globaler Medienkommunikation, der Popkultur und des Internets mannigfach beobachten lässt, übrigens auch außerhalb des Fremdsprachenunterrichts im lebensweltlichen Verhalten der jungen Menschen. (Hallet 2011: 101)

An dieser Stelle setzt das Forschungsvorhaben an, das fremdsprachliche Austausch- und Begegnungsprojekte von Schülerinnen und Schülern der gymnasialen Oberstufe anhand von schriftlichen Projektdokumentationen auf ihren inter- und transkulturellen Gehalt hin analysieren und in den Kontext der Austausch- und Begegnungsforschung einordnen will .

\section{$2 \quad$ Ansätze der Austausch- und Begegnungsforschung}

Fremdsprachlichen Austausch- und Begegnungsprojekten kommt in Zeiten von Globalisierung und Internationalisierung im schulischen Kontext eine herausgehobene Bedeutung zu. Neben den internationalen Abschlüssen, wie z. B. dem International Baccelaureat (IB) und dem bilingualen deutsch-französischen Abitur/Baccelaureat (AbiBac) gehören sie zu wichtigen Kriterien für die Schulwahl. Ehrenreich stellt fest: „Wo ,Auslandserfahrung' drauf steht, so meint man gemeinhin, stehen Flexibilität, Offenheit und Sprachkenntnisse drin. Dem Etikett ,Auslandsaufenthalt' vertraut man - zu Recht?"“(Ehrenreich 2009: 30).

Die Austausch- und Begegnungsforschung betont, dass durch die Begegnung „neue Lern- und Experimentierräume für Realbegegnungen mit dem Anderen“ geschaffen werden (Alix 1999: 35) und somit langfristig wichtige kommunikative, interkulturelle und methodisch-strategische Teilkompetenzen gefördert werden, die sich über den Unterricht allein kaum vermitteln lassen (vgl. Grau 2001). Gleichzeitig bleibt der genaue Zusammenhang zwischen Austausch- und Begegnungsprojekten und dem konkreten Erwerb dieser erwünschten Kompetenzen weitgehend unbestimmt, wie Ehrenreich betont:

Die Mobilitätsforschung ist sich darüber einig, dass diese positiven Erfahrungen und Ziele durchaus Folge eines Auslandsaufenthaltes sein können, sie ergeben sich jedoch, wie ein Blick auf detailierte Untersuchungen zeigt, nicht immer auch keineswegs automatisch. (Ehrenreich 2009: 32) 
Im Gegensatz zur sogenannten Kontakthypothese (Allport 1954), die postuliert hatte, dass direkter Kontakt zwischen Gruppen zur Veränderung attitudinaler Teilkompetenzen wie z. B. dem Abbau von Vorurteilen und Stereotypen beitragen könne, zeigen neuere Forschungen (z. B. Thomas 1996, Coleman 1996), dass solche Einstellungsveränderungen nur unter besonderen Bedingungen erreicht werden können, sich aber keinesfalls automatisch durch Austausch- und Begegnungssituationen ergeben.

Für die Austausch- und Begegnungsforschung stehen daher konkrete Gelingensbedingungen für interkulturelles Lernen in schulischen Begegnungs- und Austauschprojekten gegenwärtig im Vordergrund (vgl. Alix 1990, Boullay et al. 1995, Breitenbach 1979, Grau 2001, Krewer et al. 1995, Thomas 1988, 1996). Dazu gehört insbesondere die Frage, wie solche Austausch- und Begegnungsprojekte mit Blick auf das Zielland vorzubereiten sind, wie eine Schüler- und Handlungsorientierung jenseits einer touristischen sightseeing tour erzielt werde könne, welche Lernarrangements sich für die konkrete Begegnungssituation in besonderer Weise eignen und wie die Austausch- und Begegnungssituation wieder sinnvoll in den Fachunterricht zurückgeführt werden kann. In der Regel wird innerhalb der Austausch- und Begegnungsforschung dabei aber von einem streng interkulturellen, d. h. auf Eigenkultur und Zielkultur bezogene Begegnungssituationen ausgegangen, während stärker transkulturell orientierte Lernszenarien (z. B. Drittortprojekte, vgl. Bücking 2000, Wrobel 2004) und die sich daraus ergebenden Potentiale nur am Rande Beachtung finden. Dabei reichen heutige Austausch- und Begegnungsprojekte oftmals über eine einfache Begegnung zwischen Eigenem und Fremden in einem weitgehend touristisch geprägten Szenario hinaus (z. B. in Form von Auslandspraktika in multinationalen Unternehmen, durch Drittortprojekte und multinationale Schülerkonferenzen, wie z. B. dem European Youth Parliament sowie internationale Projektwochen) und sind aufgrund ihrer Komplexität in besonderer Weise geeignet, das Spannungsfeld zwischen inter- und transkulturellem Lernen in schulischen Begegnungskontexten $\mathrm{zu}$ verdeutlichen. Hierzu soll das Forschungsvorhaben empirisch basierte Antworten entwickeln.

\section{Das Forschungsvorhaben}

Ausgangspunkt der Betrachtungen waren fremdsprachliche Begegnungsprojekte, die von Schülerinnen und Schülern der gymnasialen Oberstufe im Rahmen der Pilotphase des CertiLingua-Exzellenzlabels für mehrsprachige, europäische und internationale Kompetenzen (www.certilingua.net), eines europäischen Verbundprojektes von 65 Schulen in 14 Ländern durchgeführt wurden. Das CertiLinguaExzellenzlabel wurde von den beteiligten europäischen Partnerländern (z. B. Niederlande, Frankreich, Italien, Finnland, Österreich sowie mehreren deutschsprachigen Ländern) vor dem Hintergrund entwickelt, neben der nationalen 
Hochschulzugangsberechtigung ein gemeinsames Instrument zu schaffen, durch das fremdsprachliche, bilinguale und interkulturelle Kompetenzen der Schülerinnen und Schüler auf vergleichbare Weise dokumentiert werden (weitere Hinweise zur Zertifizierung und zum Ablauf des Projektes finden sich unter www.certilingua.net).

Das Dissertationsvorhaben bezieht sich auf eine zweijährige Pilotphase des CertiLingua-Exzellenzlabels, die zwischen August 2007 und Juli 2009 stattfand und an der insgesamt 210 Schülerinnen und Schüler der gymnasialen Oberstufe an 29 Schulen in Nordrhein-Westfalen teilgenommen und ihre Erlebnisse und Erfahrungen während der unterschiedlichen fremdsprachlichen Begegnungsprojekte schriftlich dokumentiert haben. Ziel des empirischen Forschungsvorhabens ist es, einen vertieften Einblick in inter- bzw. transkulturelle Lernprozesse im Rahmen von fremdsprachlichen Begegnungsprojekten zu erhalten, um nachzuvollziehen, inwieweit im Sinne Hallets fremdsprachliche Begegnungsprojekte nicht nur dazu dienen können, dass Schülerinnen und Schüler in der Begegnung interkulturelle Differenzen wahrnehmen, sondern es durch die Begegnungssituation zugleich zu einer transkulturellen Bereicherung (Hallet 2011: 101) kommen kann. Dazu soll anhand des Datenbestandes nachvollziehbar gemacht werden, welche spezifischen inter-/transkulturellen (Teil-)Kompetenzen Schülerinnen und Schüler in fremdsprachlichen Begegnungsprojekten erwerben bzw. über welche kulturellen Lernerfahrungen sie im Rahmen schulischer Begegnungsprojekte berichten und in welchen Zusammenhang diese zu entsprechenden theoretischen Modellierungen (insbesondere dem Modell der intercultural communicative competence von Byram 1997) gebracht werden können. Aus der empirischen Analyse sollen sich schließlich ergänzende Hinweise für die Planung, Durchführung und Evaluation fremdsprachlicher Begegnungsprojekte entwickeln lassen. Daraus resultierende Fragestellungen sind folgende:

- Wie lassen sich die inter- und transkulturellen Lernerfahrungen der Schülerinnen und Schüler in fremdsprachlichen Begegnungsprojekten mit Hilfe fremdsprachendidaktischer Theorien und Modelle beschreiben?

- Sind die von den Schülern gemachten und in den Schülerdokumentationen dargestellten kulturellen Lernerfahrungen eindeutig einem inter- oder transkulturellen Paradigma zuweisbar?

- Wie lässt sich ggf. das interkulturelle Modell um transkulturelle Aspekte erweitern, um der Vielfalt und Hybridität schulischer Begegnungssituationen besser gerecht zu werden?

- Lassen sich aus dem Ergebnis Schlussfolgerungen für die Planung, Durchführung und Evaluation schulischer Begegnungsprojekte im Fremdsprachenunterricht ziehen? 
Mit Blick auf die Zielsetzung sowie die konkrete Fragestellung der Untersuchung bietet sich hierbei ein weitgehend exploratives und offenes Vorgehen an, bei dem nicht nur theoretische Konzepte überprüft und auf den empirischen Datenbefund angewendet werden, sondern im Sinne eines ,kontrollierten Fremdverstehens" (Flick 2005: 462) aus dem Material heraus entwickelt werden, um so zugrunde liegende ,alltagsweltliche Deutungen und Bedeutungszuweisungen“" (ebd.: 464) sichtbar zu machen. Für das Forschungsvorhaben soll weniger eine empirische Überprüfung von Hypothesen (z. B. des Kompetenzstrukturmodells der interkulturellen kommunikativen Kompetenzen nach Byram 1997), sondern stärker ein am Alltag der Schülerinnen und Schüler orientiertes sowie aus der kommunikativen Situation heraus entwickeltes Nachvollziehen bzw. Fremdverstehen inter- und transkultureller Lernerfahrungen und einer sich daraus entwickelnden Interpretation und Generierung von Hypothesen zum inter-/transkulturellen Lernen in fremdsprachlichen Begegnungssituationen im Sinne eines hybriden Begegnungsraumes im Vordergrund stehen. Die qualitative Inhaltsanalyse nach Mayring (2008) erscheint in diesem Zusammenhang als Methode zur Auswertung des empirisch gewonnenen Datenkorpus besonders geeignet, da sie sich durch eine weitgehend standardisierte Methodik auszeichnet (vgl. Flick 2005: 283) und somit eine intersubjektive Nachvollziehbarkeit sicherstellt.

Die von den Schülerinnen und Schülern im Rahmen der Qualifizierung für das Exzellenzlabel CertiLingua schriftlich verfassten Dokumentationen bilden einen einzigartigen Textkorpus zum inter- und transkulturellen Lernen in fremdsprachlichen Begegnungsprojekten in der Sekundarstufe II. ${ }^{1}$

Bereits der erste Einblick in die im Rahmen der Pilotstudie ausgewerteten Dokumentationen verdeutlicht die Bandbreite an kulturellen Erfahrungen, die Schülerinnen und Schüler in den verschiedensten Austausch- und Begegnungsprojekten sammeln können. Dabei stellt sich die oben bereits angedeutete Unterscheidung zwischen inter- und transkulturellen Lernerfahrungen als besonders relevant heraus, ohne dass es möglich erscheint, das Datenmaterial exklusiv zugunsten des einen oder anderen Paradigmas zu interpretieren. An vier (nicht repräsentativen) Textauszügen aus der Pilotierung der Daten soll dieses kurz verdeutlicht werden.

\footnotetext{
${ }^{1}$ Insgesamt standen für die Untersuchung 111 Schülerdokumentationen zur Verfügung, von denen 10\% im Rahmen einer ersten Pilotstudie zum Zweck der Erprobung des Kategorienrasters und der Kategoriendefinitionen bereits systematisch ausgewertet wurden. Durch eine angemessene Fallauswahl nach dem Prinzip des theoretical sampling (vgl. Glaser/Strauss 1998) soll die Heterogenität des Feldes angemessen abgebildet werden, um ggf. zu Verallgemeinerungen bzw. empirisch begründeten Typenbildungen zu gelangen (vgl. Kelle/Kluge 1999).
} 


\section{Beispiel 1:}

During my stay I found out that the Americans, or at least most of the ones I got to know, have a different way of thinking than us Germans. They are tending to think that they invented almost everything and are a step ahead in economics, technology and way of life. While I played soccer with a couple of friends one of them asked me how I was able to buy "Adidas" soccer cleats in Germany. When I told him that "Adidas" is originally German, they could not believe it. I don't want to call it ignorant but as soon as they are not sure about the origin of something they rather call it American than anything else which is actually not just a typical American thing to do, but it is more obvious to notice it if oneself did not grow up with this prejudices. (2009-ID59)

Dieses erste Beispiel ist typisch für eine Vielzahl von Textauszügen, in denen vor allem der kulturelle Gegensatz (z. B. Deutschland - USA oder Deutschland - GB) herausgestellt wird, ohne dass dabei eine tiefergehende Reflexion der Fremdperspektive stattfindet. Im Sinne des interkulturellen Lernens steht in diesem Zusammenhang die Wahrnehmung kultureller Differenz und deren stereotype Deutung als z. B. ,typisch amerikanisch' im Vordergrund. In ähnlicher Weise lassen sich weitere Textbeispiele finden, in der Autostereotype vor dem Hintergrund der im Zielland gemachten Erfahrungen erklärt und bekräftigt werden (d. h. die Selbstdarstellung als ,typisch deutsch' dient zur Interpretation bestimmter Sicht- und Handlungsweisen).

\section{Beispiel 2:}

The German and the American mentality differ in a lot of ways. During my year abroad I did not only learn about the American mentality, I also started to adopt some of its features. [...] Americans generally have a really laid back attitude meaning they take things the way they are and follow an attitude which could best be described as just taking it easy. Germans are the exact opposite in our opinion. They are likely to freak out about minor issues and show a rather serious and bureaucratic attitude. Everything always has to be like they are used to it and if something happens out of order many people cannot really handle it. (2009-ID26)

Auch im zweiten Beispiel dominiert die kulturkontrastive Perspektive, auch wenn sich im folgenden Beispiel ein Perspektivenwechsel (und ggf. eine Perspektivenübernahme) von der Eigen- zur Fremdkultur erkennen lässt. In ähnlicher Weise finden sich in vielen Textbeispielen Prozesse des decentering, d. h. des Infragestellens der eigenen, kulturell geprägten Position auf Basis der Erfahrungen im Zielland. Dieses decentering findet dabei aber stets auf Basis einer binären Gegenüberstellung von Eigen- und Fremdkultur statt, ohne dass dabei die eigene kulturelle Identität als vielschichtig und heterogen erkannt wird. Im Gegensatz dazu finden sich im Datenmaterial aber auch Beispiele, in denen eine transkulturelle Perspektive stärker im Vordergrund zu stehen scheint, wie im folgenden Beispiel dargestellt. 


\section{Beispiel 3:}

Joining the European Youth Parliament was the best decision that I have ever made. Not only did I get to know people from all over Europe, had lots of fun at the sessions but I also learned some skills that will help me with my future life. I learned how to work together on a team, write and present speeches in front of an audience and above all I earned a lot of self esteem. I'm thankful that I was given the chance to express my thoughts and ideas on the EU among others. I faced my fears and feel like I have truly accomplished something. Therefore I believe that the EYP truly is a chance to grow personally. After every session I'm captured by this special EYP spirit again who makes me feel truly European and who tells me that I can make an impact on Europe's future. [...] At the same time, I learned how enriching our different cultural backgrounds are with regard to our views on certain things. (2009-ID17)

Auf Basis der gemachten Erfahrungen in komplexen Begegnungsprojekten (wie hier dem European Youth Parliament) erkennen die Schülerinnen und Schüler die Heterogenität kultureller Deutungsmuster. Die Bipolarität von Eigen- und Fremdkultur wird dabei weitgehend zugunsten eines differenzierteren Blicks aufgehoben, und Schülerinnen und Schüler erleben nicht selten eine neue kulturelle Selbstwahrnehmung, wie im obigen Beispiel als „truly European“. Besonders bedeutsam erscheint in diesem Zusammenhang auch die oftmals zu beobachtende Fokussierung auf individuelle Haltungen und Einstellungen statt einer kulturell geprägten Stereotypisierung sowie die Verknüpfung mit konkreten transkulturellen Handlungen in der Fremdsprache. Gerade die Verwendung von Englisch als Lingua Franca in internationalen Begegnungsprojekten und die konkrete Handlungsorientierung dieser Projekte erleichtern scheinbar das decentering und die Wahrnehmung kultureller Heterogenität.

\section{Beispiel 4:}

The last topic we talked about was the riots in Tibet: Not only for Zhengfa was it a completely different perspective which German news presented in the media but also for Xiuqing. Both told me that they do not have subjects in China in which they discuss political or social affairs. They were astonished about our frequent vivid discussions in which we sometimes have been very incensed at Chinese politics. Whereas Zhengfa now developed a critical point of view towards his own government, Xiuqing's opinion reflected more the Chinese one. She was happy when her father told her that China controls Tibet. Consequently I asked her why she as a Chinese is proud of the obvious suppression in Tibet. She simply replied that she learned that Tibet belongs to China. Now Dalai Lama wanted to take this part away from China and he wanted to boycott the Olympic Games so that China gets a bad image. I soon realized that it is hard to make clear to her how western countries see this governmental intervention of China. She learned that her opinion is the right one and therefore does not consider anything that contradicts what her father told her. For our partnership this means that topics like these ones better should be avoided. Otherwise, friendship and partnership will be put a test which is not easy to stand. (2008-ID31) 
Das letzte Beispiel verdeutlicht, was Welsch (1992: 20) als „wechselseitiges Einarbeiten von Kulturmustern" beschreibt. Die Schülerin im oben genannten Beispiel wird zur interkulturellen Mittlerin, die zwischen den unterschiedlichen Perspektiven der beiden chinesischen Mädchen vermittelt und dabei ihre eigene, festgefügte Identität weitgehend aufgibt, um zu einem tieferen Verständnis der unterschiedlichen Sichtweisen zu gelangen. In weiteren Textpassagen interpretiert sie unterschiedliche Kulturmuster und bringt dabei auch vorsichtig die eigene Position ein, ohne dabei den Gesprächspartnern ihre Sicht aufzuzwingen. In ähnlicher Weise finden sich Beispiele, in denen die Schülerinnen und Schüler erkennen, wie kulturelle Handlungsmuster nicht nur durch die nationale Herkunft, sondern durch eine Vielzahl von Faktoren wie sozialer Herkunft, Erziehung und Bildung und vieles mehr beeinflusst werden und sich daher mitunter auch der Zielkultur (zumindest in Teilen) zugehörig fühlen können.

Die Textbeispiele verdeutlichen bereits, dass eine streng auf interkulturellen Kompetenzerwerb ausgerichte Interpretation des Datenmaterials unter Umständen zu einer verkürzten Wahrnehmung der tatsächlichen Lernerfahrungen und einer Reduktion auf streng bipolare Konzeptionen von Eigenem und Fremden führen könnte. Um die Komplexität der Daten angemessen erfassen und auswerten zu können und zugleich im Sinne Hallets (2011) sowohl auf ihre interkulturellen Differenzen als auch transkulturellen Bereicherungen hin analysieren zu können, bietet sich ein systematisches, strukturiertes, regelgeleitetes Verfahren zur Beschreibung und Kategorisierung der Daten an, dass zugleich genügend Raum für induktive Erweiterungen des von Byram (1997) beschriebenen Modells der interkulturellen kommunikativen Kompetenz um transkulturelle „Bereicherungen“ ermöglicht. Mit der Qualitativen Inhaltsanalyse scheint ein solches Auswertungsinstrument verfügbar und im beschriebenen Kontext auch angemessen anwendbar. Die Nutzung der Qualitativen Inhaltsanalyse im konkreten Forschungskontext und die dafür erforderlichen Adaptionen sollen nachfolgend kurz skizziert werden.

\section{Die Qualitative Inhaltsanalyse als Auswertungsinstrument}

Die Datenauswertung soll in Anlehnung an die Qualitative Inhaltsanalyse nach Mayring (2008) erfolgen, die den Vorteil bietet, sprachlich vermittelte Interaktion (in diesem Fall von den Schülerinnen und Schülern verfasste schriftliche Dokumentationen ihrer Austausch- und Begegnungsprojekte) so zu analysieren, dass auch latente Sinnstrukturen erfasst werden können (vgl. Lamnek 2010: 460ff.). Mayring betont: „Qualitative Inhaltsanalyse will Texte systematisch analysieren, indem sie das Material schrittweise theoriegeleitet mit am Material entwickelten Kategoriensystemen bearbeitet" (Mayring 2002: 114). Durch die Regelgeleitetheit der Analyse, die einem festen Ablaufschema folgt, wird das Textmaterial dabei in nachvollziehbarer Weise und schrittweise in einzelnen 
Analyseeinheiten untersucht und einem, theoriebasiert entwickelten Kategoriensystem zugeordnet. Daher erweist sich die Qualitative Inhaltsanalyse nicht nur für fremdsprachendidaktische Forschung als besonders fruchtbares Auswertungsinstrument (vgl. Aguado in diesem Band, Eberhardt 2010, Eberhardt in diesem Band, Haudeck 2005, Wilden 2008), sondern bietet aufgrund der Berücksichtigung von inhaltlichen Bedeutungen, die nicht explizit benannt wurden (latente Sinnstrukturen), sowie der Möglichkeit, aus dem sprachlichen Material auch „Rückschlüsse auf nichtsprachliche Phänomene“ zu ziehen (vgl. Lamnek 2010: 434), ein besonderes Potential für das geplante Forschungsvorhaben. Dadurch grenzt sich die Qualitative Inhaltsanalyse von offeneren hermeneutischen Verfahren ab, die ebenfalls um Verstehen bemüht sind, aber dabei weniger regelgeleitet vorgehen und durch ein koordinierendes Interpretieren zwischen Text und Kontext Sinnzusammenhänge extrapolieren wollen (vgl. ebd.: 469f.). Mayring betont, dass erst durch umfassende Deskription des Subjekts und des konkreten Kontextes (Einzelfallbezogenheit) sowie die ausführliche Kontrolle der verwendeten methodischen Schritte sichergestellt werden kann, dass die Forschung den konkreten Einzelfall ausreichend berücksichtigt und nicht zu vorschnellen, aus den theoretischen Vorüberlegungen abgeleiteten Hypothesen gelangt (vgl. Mayring 2008: 24f.). Daher ist die Explikation der einzelnen methodischen Verfahrensschritte und die Regelgeleitetheit der Untersuchung eine wichtige Kontrollinstanz zur Sicherstellung erforderlicher forschungsmethodischer Qualitätsstandards.

Die strukturierende Inhaltsanalyse geht nach Mayring dabei deduktiv vor, d. h. die einzelnen Strukturierungsdimensionen werden aus den theoretischen Überlegungen heraus definiert und durch entsprechende Definitionen, Ankerbeispiele (d. h. konkrete Textstellen, die dieser Kategorie entsprechen) sowie Kodierregeln (Regeln zum Umgang mit Abgrenzungsproblemen) ergänzt.

Für das konkrete Forschungsvorhaben ergibt sich hieraus allerdings ein grundsätzliches forschungsmethodisches Problem. Der Qualitativen Inhaltsanalyse nach Mayring wird von ihren Kritikern vorgehalten, dass sie sich durch ihr quantitatives und deduktives Forschungsvorgehen zwar für hypothesenüberprüfende Forschungsansätze durchaus eignen mag, für hypothesengenerierende und stärker explorativ angelegte Forschungsvorhaben aber denkbar ungeeignet sei. So kritisiert Reichertz (2003: 11), dass die Qualitative Inhaltsanalyse aufgrund der stärker quantitativen Auswertungsmethodik (insbesondere Häufigkeits- und Valenzanalysen) und dem rein deduktiven Vorgehen im Wesentlichen tautologisch sei und nicht dazu geeignet sei, neuen Erkenntnisgewinn zu erbringen. Nach Meuser (2003: 90) ist „eine Inhaltsanalyse freilich erst dann als qualitativ zu bezeichnen, wenn sie nicht klassifikatorisch, sondern sinnrekonstruierend verfährt". Die Zuordnung zu subjektiven Materials gemäß bestimmter Verfahrensregeln zu vorher definierten Kategorien sei aber aus Sicht der qualitativen Sozialforschung eben noch nicht sinnrekonstruierend, sondern bestenfalls 
klassifizierend, weil die Perspektive des Forschungssubjektes und dessen subjektive Konstruktion von Sinnstrukturen als Grundprinzip interpretativer Forschung ausgeschlossen bliebe (vgl. Früh 1998, Ritsert 1975). Und auch für das geplante Forschungsvorhaben würde ein streng deduktives Verfahren, bei dem die Hauptkategorien sowie die Ausprägungen zuvor theoriebasiert festgelegt werden, zwar eine Berücksichtigung der theoretisch bereits konkret definierten interkulturellen Kompetenzen anhand des von Byram (1997) erarbeiteten Modells der interkulturellen kommunikativen Kompetenz ermöglichen, wäre aber kaum in der Lage, das Kategoriensystem transkultureller Kompetenzen, für das noch keine explizit modellierte Struktur vorliegt, angemessen zu berücksichtigen. Erste Kodierversuche mit Hilfe der inhaltlich strukturierenden Inhaltsanalyse nach Mayring im Rahmen einer Pilotstudie blieben daher weitgehend erfolglos und konnten die Komplexität des Datenmaterials nicht angemessen abbilden.

Zwar hat Mayring inzwischen das Verfahren der Qualitativen Inhaltsanalyse um einen induktiven Ansatz erweitert, bei dem „die Kategorien direkt aus dem empirischen Material in einem Verallgemeinerungsprozess" (Mayring 2008: 75) abgeleitet werden, ohne auf theoretische Vorannahmen zu rekurrieren, doch diese empirisch ermittelten Kategorien müssen sich an einem theoriegeleitet entwickelten Selektionskriterium messen lassen. Im Gegensatz zu Mayring, der von einem deduktiven oder induktiven Ansatz ausgeht, betont Steigleder (2008: 170), dass auch eine sinnvolle Kombination beider Ansätze im Rahmen der Qualitativen Inhaltsanalyse denkbar sei. Diese kann ,je nach Thematik bzw. Forschungsgegenstand sinnvoll sein, um bestehende Theorien zu überprüfen, zu erweitern oder auch neue Theorien zu generieren" (Steigleder 2008: 170). In fremdsprachendidaktischen Forschungsarbeiten (vgl. z. B. Andringa 2004, Eberhardt in diesem Band, Wilden 2008), ${ }^{2}$ hat sich eine Kombination von deduktivem und induktiven Vorgehen durchgesetzt, bei dem die Hauptkategorien anhand eines deduktiv entwickelten Modells empirisch überprüft und durch induktiv aus dem Datenmaterial entwickelte Unterkategorien erweitert werden. Durch Abgleich mit dem Datenmaterial werden die induktiv gefundenen Unterkategorien dabei unter bereits gebildete Kategorien subsumiert, so dass die zunächst induktiv aus dem Datenmaterial entwickelten Kategorien schließlich als deduktive Kategorien gelten können. Allerdings ist diese Kombination von induktivem und deduktivem Vorgehen nach Steigleder (2008) grundsätzlich problematisch,

[...] weil erstens die durch die Theoriegeleitetheit bestimmte Systematik und Regelgeleitetheit der Interpretationstechnik der Strukturierung, die ja für sie charakteristisch ist, durch den möglicherweise induktiven Revisionsschritt aufgeweicht wird, und zweitens durch die nicht eindeutige methodische Vorgehensweise, beispielsweise das Gütekriterium der intersubjektiven Überprüfbarkeit nicht mehr gewährleistet ist. An

\footnotetext{
${ }^{2}$ Zur Problematik eines gemischt deduktiv-induktiven Vorgehens vgl. auch Bortz/Döring (2006).
} 
dieser Stelle schränkt sich die strukturierende qualitative Inhaltsanalyse ohne Not im Hinblick auf die Validität und damit die Qualität ihrer Auswertungsmethodik ein. (Steigleder 2008: 62, vgl. Bortz/Döring 2006: 330)

Es ist daher eine Modifikation des Ablaufschemas der strukturierenden Qualitativen Inhaltsanalyse nötig, um auch für das konkrete Forschungsvorhaben eine stärker explorative, sowohl an theoretischen Vorannahmen als auch am empirischen Datenbestand orientierte Vorgehensweise zu entwickeln. Steigleder (2008: 175) betont, dass gerade durch die Kombination von theorie- und empiriegeleiteter Kategorienbildung insbesondere subjektive Wahrnehmungen und latente Sinnstrukturen in den Analysedokumenten deutlicher erfasst werden können (ebd.). ${ }^{3}$ Wie eine solche Modifikation des von Mayring entwickelten Verfahrens der Qualitativen Inhaltsanalyse mit dem Ziel einer gleichberechtigten Berücksichtigung sowohl theoriegeleiteter als auch empirisch aus dem Datenmaterial entwickelter Kategorien gelingen kann und wie zugleich die Balance zwischen größtmöglicher Offenheit gegenüber dem Forschungsgegenstand und grundsätzlicher Regelgeleitetheit des Verfahrens am Beispiel des Forschungsvorhabens realisiert werden kann, soll im Folgenden kurz skizziert werden.

\section{Die Qualitative Inhaltsanalyse als iterativer und kommu- nikativer Dialog zwischen Forscher, Text und Kontext}

Vor dem Hintergrund der wesentlichen Kritikpunkte an Mayrings Verfahren der Qualitativen Inhaltsanalyse (insbesondere die unklare Kombination von induktiven und deduktiven Verfahren sowie der notwendigen Explikation erforderlicher Zwischenschritte zur Kontrolle und ggf. Revision des Kategoriensystems; vgl. Gläser/Laudel 2004, Steigleder 2008: 185) entwickelt Steigleder (2008) ein alternatives Ablaufmodell einer ,theorie- und empiriegeleiteten Kategorienbildung“ (ebd.: 189), das sich für das hier vorgestellte, explorative Vorgehen als besonders hilfreich erwiesen hat, weil durch die Konkretisierung der Zwischenschritte nicht nur eine Kombination deduktiver (d.h. aus der Theorie zum inter- und trans-

\footnotetext{
${ }^{3}$ Gerade im vorliegenden Forschungsvorhaben geht es nicht um objektive bzw. objektivierbare Wahrnehmungen inter- und transkultureller Lernerfahrungen, sondern um subjektive Deutungsprozesse bzw. um ein Fremdverstehen individueller semantisch-indexikaler Relevanzsysteme der Schülerinnen und Schüler (vgl. Bohnsack 2000: 20, Kruse 2009: Abs. 18), die nur vom Forschenden erfasst werden können, wenn dieser sich eine grundsätzliche Offenheit gegenüber dem Forschungsgegenstand vorbehält und sowohl theoriegeleitete als auch empirisch entwickelte Überlegungen in den Deutungsprozess mit aufnimmt. Theoriegeleitet meint in diesem Zusammenhang, das eigene theoretisch fundierte Vorwissen für den Erkenntnisprozess zu nutzen, ohne dass dieser dadurch determiniert würde. Die Grounded Theory (Strauss/Corbin 1996: 56ff.) spricht in diesem Zusammenhang von ,theoretischer Sensibilität' als einer „Verfügbarkeit brauchbarer heuristischer Konzepte, die die Identifizierung theoretisch relevanter Phänomene im Datenmaterial ermöglicht“" (Kelle 1996: 32).
} 
kulturellen Lernen abgeleiteter Kriterien) und induktiver (d. h. konkret aus dem Datenmaterial extrapolierter Kriterien) möglich wurde, sondern zugleich eine Kontrolle wesentlicher Verfahrensschritte durch am Projekt beteiligte Lehrerinnen und Lehrer schon während des Auswertungsprozesses möglich wurde. Im Prozess erwies sich die Qualitative Inhaltsanalyse weniger als ein lineares Vorgehen nach einem klar umrissenen Ablaufschema (wie von Mayring 2008 beschrieben), sondern zeigte sich vielmehr als ein iterativer und kommunikativer Dialog des Forschers mit dem eigenen Theorieverständnis und den eignen Vorannahmen sowie dem konkret im Datenbestand aufgefundenen empirischen Befund und dem kontextuellen Abgleich durch kommunikative Validierung mit Projektbeteiligten. Nachfolgend wird das für das Forschungsvorhaben konkretisierte Ablaufschema kurz dargestellt (Tab. 1), wobei zunächst die Grundzüge des Ablaufmodells nach Steigleder (2008) sowie die eigenen Adaptionen für das Forschungsprojekt skizziert werden, während Abbildung 2 den iterativen Prozess zwischen theoriegeleiter Forscherperspektive, empiriegeleiteter Textperspektive und der dialogbasierten Kontextperspektive zu verdeutlichen sucht.

Tabelle 1: Konkretisierung des Ablaufschemas in Anlehnung an Steigleder (2008: 166, 186f.)

\begin{tabular}{|l|l|}
\hline Schritt & Analyseschritte des modifiziertes Ablaufschemas \\
\hline $\mathbf{1}$ & $\begin{array}{l}\text { Vorbereitungsphase: mehrmaliges Lesen des Datenmaterials und Entwicklung } \\
\text { einer Strategie zur Bearbeitung des Datenmaterials (hier insbesondere Berück- } \\
\text { sichtigung des theoretical sampling, sequentielle und kontextorientierte } \\
\text { Auswertung der Schülerdokumentationen). Digitalisierung der Schülerdokumente } \\
\text { und Einarbeitung der Daten in das Programm MAXQDA. }\end{array}$ \\
\hline $\mathbf{2}$ & $\begin{array}{l}\text { Bestimmung der Analyseeinheiten: textbasierte Vorstrukturierung der Schülertexte } \\
\text { in Sinnabschnitte (z. B. Projektdarstellung, Begegnungssituationen, Projektreflexion) }\end{array}$ \\
\hline $\mathbf{3}$ & $\begin{array}{l}\text { Bildung von Strukturierungsdimensionen: Entwicklung von inter-und } \\
\text { transkulturellen Hauptkategorien auf Basis des Modells von Byram (1997), } \\
\text { theoretischen Überlegungen zum Konstrukt des ,transkulturellen Lernens' (3a) } \\
\text { und ersten empiriegeleiteten Datenauswertungen durch Berücksichtigung } \\
\text { inhaltlicher Sinnabschnitte und Schlagworte im Text (3b). }\end{array}$ \\
$\begin{array}{l}\text { Bestimmung von Ausprägungen: theoriegeleitete Entwicklung von Ausprägungen } \\
\text { zu den inhaltlichen Hauptkategorien des inter- und transkulturellen Lernens (4a), } \\
\text { empiriegleitete Erweiterung der Ausprägungen (4b) und Zusammenstellung eines } \\
\text { gemeinsamen Kategoriensystems. Überprüfung anhand des bisher kodierten } \\
\text { Datenmaterials durch kommunikative Validierung mit projektbeteiligten Lehrerinnen } \\
\text { und Lehrern, ggf. Überarbeitung und Zusammenstellung eines Kategoriensystems } \\
\text { aus Haupt- und Unterkategorien (4c). }\end{array}$ \\
\hline
\end{tabular}

\footnotetext{
${ }^{4}$ MAXQDA ist ein Programm zur computergestützten Analyse qualitativer Daten, das sich insbesondere für den Umgang mit größeren Datenbeständen und durch die vielfältigen Funktionen zur Datenanalyse und Auswertung bewährt hat (www.maxqda.de); zur Nutzung von QDA-Software für qualitative Textanalysen vgl. Kuckartz (2005).
} 


\begin{tabular}{|c|c|}
\hline 5 & $\begin{array}{l}\text { Erstellung eines Kodierleitfadens: Festlegung von theoriebasierten / } \\
\text { empiriebasierten Kategoriendefinitionen (5a/b) als inhaltliche Konkretisierung } \\
\text { einer Kategorie sowie entsprechender Kodierregeln (Bestimmung der Reichweite } \\
\text { einer Kategorie). Kommunikative Validierung des Kodierleitfadens im Team } \\
\text { (5c), gemeinsame Festlegung von Ankerbeispielen für die einzelnen sowie } \\
\text { Regeln im Umgang mit einer Restkategorie (d. h. entweder nicht erkenntnis- } \\
\text { relevantes Material oder Datenmaterial, das sich mit dem Kodierleitfaden noch } \\
\text { nicht eindeutig zuordnen lässt) }\end{array}$ \\
\hline 6 & $\begin{array}{l}\text { Materialdurchlauf (Fundstellenbezeichnung): Einteilung des zu } \\
\text { analysierenden Datenmaterials entsprechend dem Kodierleitfaden in theorie- und } \\
\text { empiriegeleitete Kategorien (Schritt } 6 \mathrm{a} / \mathrm{b}) \text {, nicht einteilbares Material wird in } \\
\text { eines Restekategorie }^{5} \text { aufgenommen und getrennt überprüft (6c). Während des } \\
\text { Kodierprozesses findet eine kontinuierliche Überprüfung des Kategorienschemas } \text { statt; neue, induktiv ermittelte Kategorien werden in das Kategorienschema } \\
\text { aufgenommen und am Datenmaterial überprüft (6d). }\end{array}$ \\
\hline 7 & $\begin{array}{l}\text { Überprüfung der Kategorien: Nach dem Durchgang durch das Datenmaterial } \\
\text { und Abschluss der Überarbeitung werden die Fundstellen theoriebasiert auf } \\
\text { Plausibilität und Passung überprüft (7a) und das Kategoriensystem ggf. um } \\
\text { empiriegeleitete Kategorien erweitert (7b). Die Kodierung wird durch eine } \\
\text { externe Validierung (Zweitkodierung und Bestimmung der Interrater-Reliabilität) } \\
\text { überprüft (7c). Nach Überprüfung der Restekategorie werden Kategorien } \\
\text { inhaltlich oder begrifflich konkretisiert und präzisiert. Falls möglich, werden } \\
\text { Ober- und Unterkategorien zum inter- und transkulturellen Lernen } \\
\text { zusammengefasst und abstrahiert. }\end{array}$ \\
\hline 8 & $\begin{array}{l}\text { Datenauswertung und Interpretation: Die Ergebnisse der Datenauswertung } \\
\text { werden deskriptiv dargestellt und vor dem Hintergrund der Forschungsfragen } \\
\text { interpretiert. Der gesamte Forschungsprozess wird einer kritischen Reflexion } \\
\text { unterzogen (insbesondere der Ertrag des methodischen Vorgehens, mögliche } \\
\text { Schwachstellen der Forschungsmethodik der QIA und der vorgenommenen } \\
\text { Adaptionen werden thematisiert). Abschließend werden aus dem Forschungs- } \\
\text { prozess ableitbare Konsequenzen für schulische Austausch- und } \\
\text { Begegnungsprozesse und ihre Bedeutung für inter- und transkulturelles Lernen } \\
\text { im Fremdsprachenkontext formuliert. }\end{array}$ \\
\hline
\end{tabular}

${ }^{5}$ Die Schülerinnen und Schüler beschreiben z. T. in ihren Dokumentationen sehr umfangreich den Ablauf des Begegnungsprojektes oder ihren typischen Alltag im Auslandssemester, ohne dass sich hier konkrete inter- oder transkulturelle Erfahrungen ablesen ließen. Diese Textteile werden zunächst in eine Restekategorie aufgenommen, und es wird zum Abschluss der Kodierung noch einmal überprüft, ob sich hier ggf. weitere, erkenntnisrelevante Unterkategorien finden lassen oder ob das Material für die Forschungsfrage irrelevant ist und daher aussortiert werden kann. 
theoriegeleitete

Forscherperspektive empiriegeleitete

Textperspektive dialogbasierte

Kontextperspektive

1 Vorbereitungsphase : mehrmaliges Lesen des Datenmaterials und

1 Aufbereitung der Daten für die computergestützte Weiterverarbeitung
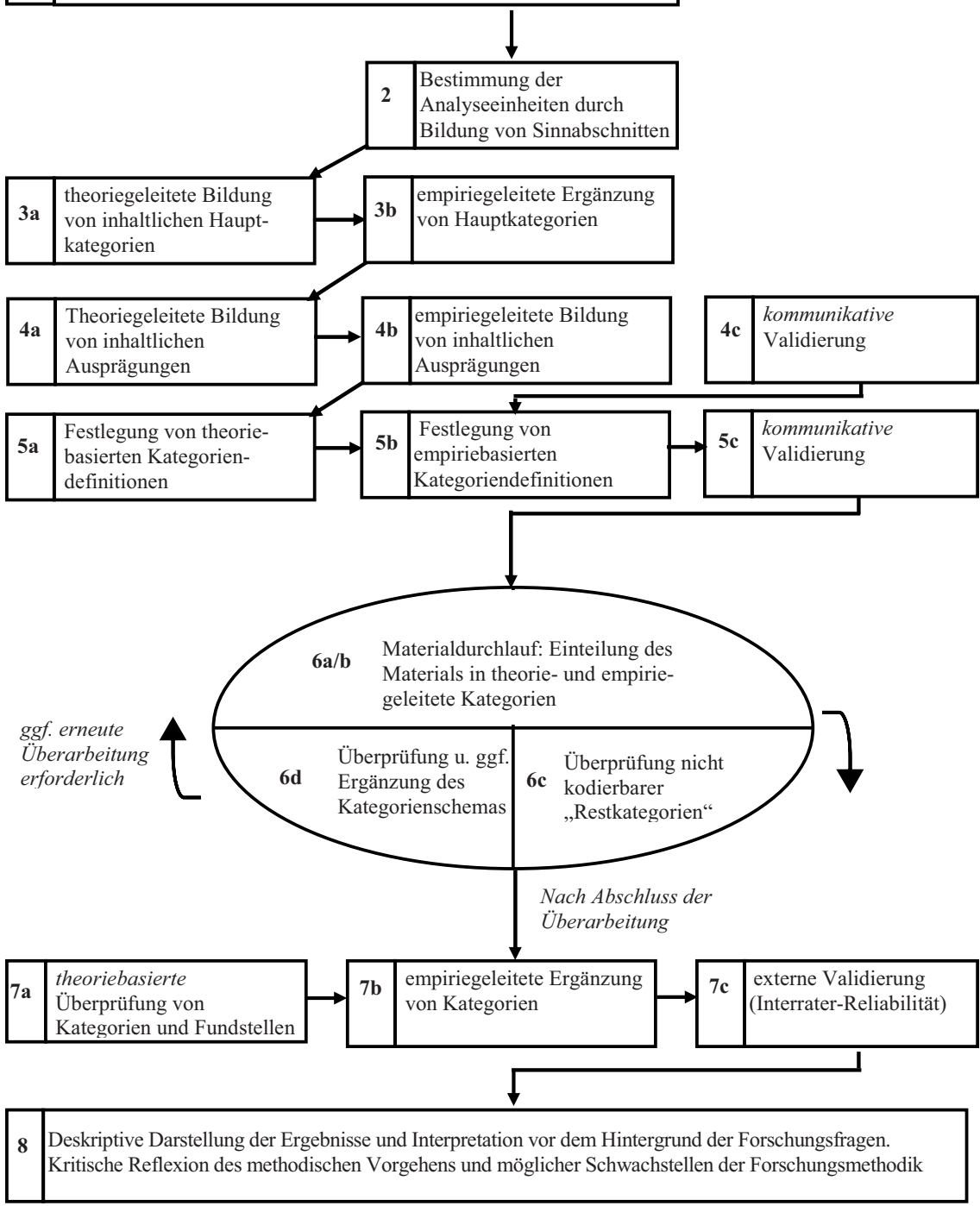

Abbildung 2: Die Qualitative Inhaltsanalyse als iterativer und kommunikativer Dialog zwischen Forscher, Text und Kontext. Quelle: eigene Erstellung, in Anlehnung an Steigleder (2008: 189) 
Das von Steigleder (2008) vorgeschlagene und in Abbildung 2 für das konkrete Forschungsvorhaben angepasste Ablaufschema für die Qualitative Inhaltsanalyse kann der grundsätzlichen Kritik am Verfahren standhalten und belegt, dass die Qualitative Inhaltsanalyse - bei entsprechender Adaption ${ }^{6}$ des Ablaufschemas im Sinne eines stärker iterativen und kommunikativen Vorgehens (d. h. durch Wechsel zwischen theorie- und empiriebasierten Perspektiven auf den Datenbestand und durch regelmäßige kommunikative Validierung von Zwischenergebnissen) sicherstellen kann, dass die Auswertung des Datenmaterials sowohl theoriegeleitet als auch empiriebasiert erfolgt und Überprüfungen und Anpassungen des Kategoriensystems an die sich verändernde Datenlage jederzeit möglich sind.

Damit eignet sich die Qualitative Inhaltsanalyse durchaus auch für stärker explorativ-interpretative Forschungsansätze, die insbesondere für die Fremdsprachendidaktik von herausgehobener Bedeutung sind (vgl. Caspari et al. 2003), sofern dabei grundsätzliche Prinzipien wie Offenheit (gegenüber Vorannahmen und Einstellungen des Forschers), Flexibilität (im Umgang mit dem Untersuchungsdesign), Kommunikativität (Berücksichtigung weiterer Perspektiven, z. B. von am Forschungsprozess Beteiligten) und Reflexivität (kritische Wahrnehmung der eigenen Subjektivität des Forschers) Berücksichtigung finden (vgl. Steinke 1999).

\section{Literatur}

Alix, Christian (1990). „Pakt mit der Fremdheit?“ - Interkulturelles Lernen als dialogisches Lernen im Kontext internationaler Schulkooperationen. Frankfurt a.M.: Verlag für internationale Kommunikation.

Alix, Christian (1999). Arbeit am Fremden und Eigenen - Lernraum internationale Schulkooperation. Der Fremdsprachliche Unterricht Französisch 5: 35-38.

Allport, G. (1954). The nature of prejudice. Cambridge, MA: Perseus Books.

Altmayer, Claus (2004). Kultur als Hypertext. Zu Theorie und Praxis der Kulturwissenschaft im Fach Deutsch als Fremdsprache. München: Iudicum.

Andringa, E. (2004). The interface between fiction and life: patterns of identification in reading autobiographies. Poetics Today 25 (2): 205-240.

Antor, Heinz (2006) (Hrsg.). Inter- und Transkulturelle Studien. Theoretische Grundlagen und interdisziplinäre Praxis. Heidelberg: Winter.

Belz, Julie A. (2003). Linguistic perspectives on the development of intercultural competence telecollaboration. In: Language Learning \& Technology 7.2: 68-117.

\footnotetext{
${ }^{6}$ Eine Adaption des Verfahrens der Qualitativen Inhaltsanalyse an die Rahmenbedingungen des konkreten Forschungsvorhabens wird von Mayring selbst ausdrücklich empfohlen (vgl. Mayring 2008: 43).
} 
Bohnsack, Ralf (2000). Rekonstruktive Sozialforschung. Opladen: Leske \& Budrich.

Bortz, Jürgen / Döring, Nicola (2006). Forschungsmethoden und Evaluation für Human- und Sozialwissenschaftler. Heidelberg: Springer, 4. Aufl.

Boullay, Peter / Kiefer, Gerd / Schneider, Erich (Hrsg.) (1995): Interkulturelles Lernen im Schüleraustausch. Saarbrücken. Bd.2. Landesinstitut für Pädagogik und Medien.

Bredella, Lothar (1999). Zielsetzungen interkulturellen Fremdsprachenunterrichts. In : Bredella, Lothar / Delanoy, Werner (Hrsg.). Interkultureller Fremdsprachenunterricht: Das Verhältnis von Fremden und Eigenem. Tübingen: Narr.

Bredella, Lothar (2010). Trans- oder Interkulturalität als Bildungsziel des Fremdsprachenlehrens und -lernens? In: Forum Sprache 4/2010: 21-42.

Bredella, Lothar / Christ, Herbert (1995). Didaktik des Fremdverstehens im Rahmen einer Theorie des Lehrens und Lernens fremder Sprachen. In: Bredella, Lothar / Christ, Herbert (Hrsg.): Didaktik des Fremdverstehens. Tübingen: Narr. 8-19.

Breitenbach, Dieter (1979) (Hrsg.). Kommunikationsbarrieren in der internationalen Jugendarbeit: Ein Forschungsbericht im Auftrage des Bundesministeriums für Jugend, Familie und Gesundheit. 5 Bde. Saarbrücken / Fort Lauerdale: Breitenbach.

Bruner, Jerome (1990). Acts of meaning. Cambridge, Mass.: Harvard UP.

Bücking, Gisela (2000). Unterwegs nach Europa: Bericht über eine trinationale Jugendbegegnung in Kreisau, Polen. Fremdsprachenunterricht 44/53 (2): 135-138.

Byram, Michael (1997). Teaching and assessing intercultural communicative competence. Clevedon: Multilingual Matters.

Byram, Michael / Risager, Karen (1999). Language Teachers, politics and cultures. Clevedon: Multilingual Matters.

Caspari, Daniela / Helbig, Gerhard / Schmelter. Lars (2003). Forschungsmethoden: Explorativ-interpretatives Forschen. In: Bausch, Karl-Richard / Christ, Herbert / Krumm, HansJürgen. (Hrsg.): Handbuch Fremdsprachenunterricht. Tübingen: Francke. 449-506.

Coleman, James A. (1996). The current state of knowledge concerning student residence abroad. In: Parker, Gabrielle / Rouxeville, Annie (eds.): The year abroad. Preparation, monitoring, evaluation. Current research and development. London: Centre for Information on Language Teaching and Research.

Council of Europe (2001). Common European Framework of Reference for Languages. Learning, teaching, assessment. Cambridge: Cambridge University Press.

Delanoy, Werner (2006). Transculturality and (inter-)cultural learning in the EFL classroom. In: Delanoy, Werner / Volkmann, Laurenz (eds.): Cultural studies in the EFL classroom. Heidelberg: Winter. 233-248.

Eberhardt, Jan-Oliver (2010). Interkulturelle Kompetenz als Bildungsstandard im Fremdsprachenunterricht: zwischen Anspruch und Wirklichkeit. In: Ahrenholz, Bernt / Altmayer, Claus / Mehlhorn, Grit / Neveling, Christiane / Schlüter, Norbert (Hrsg.): Grenzen überschreiten: fachlich - sprachlich - kulturell. Dokumentation zum 23. Kongress für Fremdsprachendidaktik. Baltmannsweiler: Schneider Hohengehren

Eckerth, Johannes / Wendt, Michael (Hrsg.) (2003). Interkulturelles und transkulturelles Lernen im Fremdsprachenunterricht. Frankfurt / Main : Lang. 
Ehrenreich, Susanne (2009). Auslandsaufenthalte quer gedacht - aktuelle Trends und Forschungsaufgaben. Anmerkungen aus deutscher Warte. In: Ehrenreich, Susanne / Woodman, Gill / Perrefort, Marion (Hrsg.): Auslandsaufenthalte in Schule und Studium. Bestandsaufnahmen aus Forschung und Praxis. Münster: Waxmann. 29-38.

Fäcke, Christiane (2006). Transkulturalität und fremdsprachliche Literatur. Eine empirische Studie zu mentalen Prozessen von primär mono- und bikulturell sozialisierten Jugendlichen. Frankfurt am Main: Lang.

Flick, Uwe (2005). Qualitative Sozialforschung. Eine Einführung. Reinbek: Rowohlt.

Freitag, Britta (2010). Interkulturelle Kommunikative Kompetenz. In: Surkamp, Carola (Hrsg.): Metzler Lexikon Fremdsprachendidaktik. Stuttgart: Metzler. 121f.

Freitag-Hild, Britta (2009). Theorie, Aufgabentypologie und Unterrichtspraxis inter- und transkultureller Literaturdidaktik. 'British Fictions of Migration' im Fremdsprachenunterricht. Trier: WVT.

Früh, Werner (1998). Inhaltsanalyse. Theorie und Praxis. Unveränderte 5. Aufl. Konstanz: UVK Verlagsgesellschaft.

Glaser, Barney G. / Strauss, Anselm (1998). Grounded Theory. Strategien qualitativer Forschung. Bern: Huber.

Gläser, Jochen / Laudel, Grit (2004). Experteninterviews und qualitative Inhaltsanalyse. Wiesbaden: VS Verlag für Sozialwissenschaften.

Grau, Maike (2001). Arbeitsfeld Begegnung. Eine Studie zur grenzüberschreitenden Lehrertätigkeit in europäischen Schulprojekten. Tübingen: Narr.

Hallet, Wolfgang (2002). Fremdsprachenunterricht als Spiel der Texte und Kulturen. Intertextualität als Paradigma einer kulturwissenschaftlichen Didaktik. Trier: WVT.

Hallet, Wolfgang (2011). Lernen fördern: Englisch. Kompetenzorientierter Unterricht in der Sekundarstufe I. Seelze: Klett-Kallmeyer.

Haudeck, Helga (2005). Wie pauken Schüler und Schülerinnen Vokabeln für den Fremdsprachenunterricht wirklich? Ein Anwendungsbeispiel der Qualitativen Inhaltsanalyse aus der Lernstrategieforschung. In: Mayring, Philipp / Gläser-Zikuda, Michaela (Hrsg.): Die Praxis der Qualitativen Inhaltsanalyse. Weinheim: Beltz. 84-104.

Herder, Johann Gottfried (1984). Johann Gottfried Herder. Werke. Bd. 1. Herder und der Sturm und Drang (1764-1774). München: Hanser.

Hessisches Kultusministerium (2011). Bildungsstandards und Inhaltsfelder. Das neue Kerncurriculum für Hessen - Sekundarstufe I - Gymnasium: Moderne Fremdsprachen. Online: http://tinyurl.com/7al8tk3 (letzter Aufruf [28.2.2012]).

Kelle, Udo (1996). Die Bedeutung theoretischen Vorwissens in der Methodologie der Grounded Theory. In: Strobl, Rainer / Böttger, Andreas (Hrsg.): Wahre Geschichten? Zu Theorie und Praxis qualitativer Interviews. Baden-Baden: Nomos. 23-47.

Kelle, Udo / Kluge, Susann (1999). Vom Einzelfall zum Typus. Fallvergleich und Fallkontrastierung in der qualitativen Sozialforschung. Opladen: Leske \& Budrich.

Kramsch, Claire (1998). The priviledge of the intercultural speaker. In: Byram, Michael / Fleming, Michael (eds.): Language learning in intercultural perspective. Approaches through drama and ethnography. Cambridge: University Press. 16-31. 
Krewer, Bernd / Bohlender, Gudrun / Richter, Stefan (1995). Evaluation unter kultur- und entwicklungspsychologischen Gesichtspunkten. In: Boullay, Peter / Kiefer, Gerd / Schneider, Erich (Hrsg.): Interkulturelles Lernen im Schüleraustausch. Saarbrücken. Bd.2. Landesinstitut für Pädagogik und Medien, 27-123.

Kruse, Jan (2009). Qualitative Sozialforschung- interkulturell gelesen. Die Reflexion der Selbstauslegung im Akt des Fremdverstehens. Forum Qualitative Sozialforschung 10(1), Art. 16. Online: http://nbn-resolving.de/urn:nbn:de:0114-fqs0901162 (letzter Aufruf [28.2.2012]).

Kuckartz, Udo (2005). Einführung in die computergesteuerte Analyse qualitativer Daten. Wiesbaden: VS Verlag für Sozialwissenschaften.

Lamnek, Siegfried (2010). Qualitative Sozialforschung: Lehrbuch. Weinheim / Basel: Beltz.

Latour, Bruno (2007). Eine neue Soziologie für eine neue Gesellschaft. Frankfurt a.M.: Suhrkamp.

Mayring, Philipp (2002). Einführung in die Qualitative Sozialforschung. Weinheim/Basel: Beltz, 5. Aufl.

Mayring, Philipp (2008). Qualitative Inhaltsanalyse. Grundlagen und Techniken. Weinheim/Basel: Beltz, 10. Aufl.

Meuser, Michael (2003). Inhaltsanalyse. In: Bohnsack, Ralf / Marotzki, Wilfried, Meuser (Hrsg.): Hauptbegriffe Qualitativer Sozialforschung. Opladen: Leske und Budrich. 31-33.

Müller-Hartmann, Andreas / Schocker-von Ditfurth, Marita (2008). Field trips als Lernarrangements. Zur Ausbildung interkultureller Vermittlungskompetenz bei angehenden Englischlehrkräften. In: Ehrenreich, Susanne / Woodman, Gill / Perrefort, Marion (Hrsg.): Auslandsaufenthalte in Schule und Studium. Bestandsaufnahmen aus Forschung und Praxis. Münster: Waxmann. 123-138.

Reichertz, Jo (2003). Abduktion. In : Bohnsack, Ralf / Marotzki, Winfried /Meuser, Michael (Hrsg.): Hauptbegriffe Qualitativer Sozialforschung. Opladen: Leske und Budrich. 11-14.

Ritsert, Jürgen (1975). Inhaltsanalyse und Ideologiekritik. Ein Versuch über kritische Sozialforschung. Frankfurt a.M.: Athenäum-Fischer.

Schulze-Engler, Frank (2006). Von ,Inter' zu ,Trans': Gesellschaftliche, kulturelle und literarische Übergänge. In: Antor, Heinz (Hrsg.): Inter- und Transkulturelle Studien. Theoretische Grundlagen und interdisziplinäre Praxis. Heidelberg: Winter. 41-54.

Steigleder, Sandra (2008). Die strukturierende qualitative Inhaltsanalyse im Praxistest. Eine konstruktiv kritische Studie zur Auswertungsmethodik von Philipp Mayring. Marburg: Tectum.

Steinke, Ines (1999). Kriterien qualitativer Forschung. Ansätze zur Bewertung qualitativempirischer Sozialforschung. Weinheim, München: Juventa.

Straub, Jürgen (1999). Verstehen, Kritik, Anerkennung. Das Eigene und das Fremde in der Erkenntnisbildung interpretativer Wissenschaften. Göttingen: Wallenstein.

Strauss, Anselm / Corbin, Juliet (1996). Grounded Theory. Grundlagen qualitativer Sozialforschung. Weinheim: Beltz.

Tajfel, Henri (1982). Gruppenkonflikt und Vorurteil. Bern etc.: Huber. 
Thomas, Alexander (1988). Interkulturelles Handeln im Schüleraustausch. Saarbrücken/Fort Lauderdale: Breitenbach.

Thomas, Alexander (1996). Können interkulturelle Begegnungen Vorurteile verstärken? In: Thomas, Alexander (Hrsg.): Psychologie und multikulturelle Gesellschaft. Göttingen: Hogrefe. 227-238.

Vasilache, Andreas (2003). Interkulturelles Verstehen nach Gadamer und Focault. Frankfurt a.M.: Campus.

Weinert, Franz E. (Hrsg.) (2001). Leistungsmessung in Schulen. Weinheim: Beltz.

Welsch, Wolfgang (1992). Transkulturalität - Lebensformen nach der Auflösung der Kulturen. Information Philosophie 2: 5-20.

Welsch, Wolfgang (1994). Transkulturalität. Lebensformen nach der Auflösung der Kulturen. In: Luger, Kurt / Renger, Rudi (Hrsg.): Dialog der Kulturen. Die multikulturelle Gesellschaft und die Medien. Wien: Österreichischer Kunst- und Kulturverlag. 147-169.

Welsch, Wolfgang (2010). Was ist eigentlich Transkulturalität. In: Darowska, Lucyna / Machold, Claudia / Lüttenberg, Thomas (Hrsg.): Hochschule als transkultureller Raum? Beiträge zu Kultur, Bildung und Differenz. Bielefeld: Transcript. 39-66.

Wilden, Eva (2008). Selbst- und Fremdwahrnehmung in der interkulturellen Onlinekommunikation. Das Modell der ABC's of Cultural Understanding and Communication Online. Eine qualitative Studie. Frankfurt a.M.: Lang.

Wrobel, Jürgen (2004). Begegnung am dritten Ort. Das Drei-Länder-Begegnungsprojekt der Carlo-Mierendorff-Schule. Der Fremdsprachliche Unterricht Englisch 70: 16-21. 


\title{
Die qualitative Inhaltsanalyse als transdisziplinäres Verfahren zur Auswertung von Leseprotokollen
}

\section{Annegret Beier}

\begin{abstract}
Qualitative Content Analysis is a common method for analyzing empirical data in social science research. The following article demonstrates the application of this method to a research question in the field of German as a foreign language and the adaptation of Qualitative Content Analysis to visual data. The study is based on visual data produced by students at the World Language University of Tashkent, Uzbekistan, after reading literary texts as homework. The purpose of these data was to determine: (a) whether the connection with visual art improves understanding of literary texts, and if so, (b) which combination of these two media is the most optimal one in classroom teaching.
\end{abstract}

\section{Bilddidaktisches Handeln im Literaturunterricht DaF - Hintergrund des Forschungsprojekts}

\subsection{Bildmedien im Fremdsprachenunterricht}

In der Fremdsprachendidaktik wurde seit den 1980er Jahren das Potenzial von (Kunst-)Bildern (wieder-)entdeckt und für den Unterrichtseinsatz aufgewertet (vgl. Hecke/Surkamp 2010: 21f., Reinfried 1992: 277ff.). Spätestens mit der Debatte um visual literacy Mitte der 1990er Jahre, die die Bildkompetenz als Kommunikationsform und Teilkompetenz im Multiliteralitätsansatz aufwertete (vgl. Bach/Breidbach 2009: 296, Boehm 2001: 13, Hecke/Surkamp 2010: 21f., Legutke 2009: 207), gehört es zu den Anforderungen an eine Lehrkraft, sich fächerübergreifend mit verschiedenen Didaktiken auseinanderzusetzen.

Zwar stellen die Kunst- und Bildwissenschaft, Kunstpädagogik, Ethnografie und Fremdsprachendidaktik methodische Herangehensweisen an Bildmedien bereit (vgl. Hallet 2010, Rymarczyk 2007: 335, Seidl 2007,Thomson 2010, Uhlig 2005), jedoch ist weiterhin ungelöst, wie sich diese im Sinne des Multiliteralitätsansatzes in den Fremdsprachenunterricht überführen lassen.

Während meiner Unterrichtstätigkeit in Usbekistan fiel mir auf, dass sich die Studierenden zwar motiviert mit (Kunst-)Bildern beschäftigten, sich Bildarbeit im Fremdsprachenunterricht jedoch in der Regel auf das Auslösen von Sprachhandlungen oder Illustrieren von Texten beschränkte. Parallel zu den erwarteten interkulturellen Interferenzen beim Deuten von (Kunst-)Bildern beobachtete ich, dass die Studierenden über eine eingeschränkte Bilderfahrung verfügten. Dieses Zusammentreffen unterschiedlicher Seherfahrungen, das heißt meiner als im europäischen Kontext sozialisierten Kunsthistorikerin und der der usbekischen Studierenden, sowie die anfänglichen Misserfolge mehrkanaligen Arbeitens (mit Kunstbildern und literarischen Texten) führten zur Zielsetzung meines For- 
schungsprojekts, sich mit dem bilddidaktischen Handeln im Literaturunterricht Deutsch als Fremdsprache (DaF) zu befassen.

Über einen Zeitraum von sechs Monaten wurde der Einsatz von Kunstbildern im Literaturunterricht DaF in zwei Gruppen des dritten Studienjahres der Fakultät für Deutsche Philologie der Weltsprachenuniversität Taschkent, Usbekistan, untersucht und bewertet.

\subsection{Fragestellung der Untersuchung}

Katrin Thomson geht in ihrer Studie (2010) zu Ernest Hemingways Kurzgeschichte Cat in the Rain davon aus, dass die kategoriengeleitete Rezeption des Gemäldes Edward Hoppers Room in New York die Aufmerksamkeit für Bilddetails schult (Thomson 2010: 202). Die Verbindung der Bilddetails könne laut Thomson sowohl auf die Bedeutung des Gesamtbildes bezogen als auch aufgrund der Analogie von Wahrnehmungs- und Deutungsprozessen visueller und textsprachlicher Medien auf den literarischen Text übertragen werden (ebd.). Thomsons Ziel ist es, durch das visuelle Anschauungsmaterial eine ,gründlichere Textrezeption“ und ein „tieferes Textverständnis“ zu erreichen (ebd.).

In ihrer Studie lesen die Lernenden den Text Hemingways zunächst und behandeln im Anschluss das Gemälde Hoppers anhand von sich erweiternden Bildausschnitten. Nach der zweiten Lektüre des Textes werden die schriftlich beantworteten Fragen zum Text der 20 Lernenden mit denen nach dem ersten Lesen verglichen (ebd.: 203ff.).

Katrin Thomson greift in ihrer Studie auf die im Fremdsprachenunterricht lange bekannte Anschauungsmethode zurück (Hecke/Surkamp 2010: 20f., Reinfried 1992: 41ff.), entwickelt diese jedoch weiter, indem sie das Sehen des Bildes verlangsamt und steuert. Die Deutungsoffenheit des (Kunst-) Bildes, die in den 1920er Jahren zum zwischenzeitlichen Rückgang der Anschauungsmethode geführt hatte (ebd.: 183, 188), wird durch diese Vorgehensweise eingeschränkt.

Thomson untersucht die Veränderung des Textverständnisses innerhalb einer Gruppe, in der der Text zunächst ohne, beim zweiten Mal mit Bild behandelt wird. Fraglich ist, ob eine Gruppe, in der der Text zweimal (ohne Bild) mit unterschiedlichen literaturdidaktischen Ansätzen besprochen wird, nicht zu einem ähnlichen Ergebnis gelangt wäre. Zudem geht sie von einer Gruppe mit ähnlicher Seherfahrung aus, die sowohl ihrer als auch denen des eingesetzten Textes und Bildes vergleichbar sind.

Die Auseinandersetzung mit dieser Studie und meinen Unterrichtserfahrungen in der zentralasiatischen Republik Usbekistan führte zur Forschungsfrage, ob der Einsatz von Kunstbildern im Literaturunterricht $\mathrm{DaF}$ zu einem tieferen Ver- 
ständnis literarischer Texte führen kann. Unter ,tieferem Verständnis` wird sowohl der Grad der Textrezeption als auch die Erfassung von und Positionierung zu mehreren, am Text validierbaren Textauslegungen verstanden.

In meiner Untersuchung wurden fünf literarische Texte in den beiden Vergleichsgruppen im Kreuzdesign, das heißt jeweils mit und ohne Kunstbildeinsatz, behandelt. Im Verlauf des Untersuchungszeitraums wurden die in der Fachliteratur vorgeschlagenen Kriterien für die Text- und Bildauswahl überprüft und revidiert, Sehschulungen der Studierenden durchgeführt und unterschiedliche Didaktisierungen von Kunstbildern und literarischen Texten erprobt.

Auffällig war, dass die Studierenden zunehmend besser mit Kunstbildern umgehen konnten, das Textverständnis zwischen den beiden Gruppen jedoch weiterhin differierte. Diese Beobachtungen bildeten den Auslöser für die zweite Forschungsfrage, wie die Variablen für einen erfolgreichen Kunstbildeinsatz im Literaturunterricht $\mathrm{DaF}$ gestaltet sein müssen.

Der Kunstbildeinsatz im Literaturunterricht DaF wurde in der Untersuchung dann als erfolgreich betrachtet, wenn das eingesetzte Kunstbild nicht als Illustration zum Gesamttext oder zu textlichen Elementen verstanden wurde, sondern mit dem Kunstbild Analogien zum literarischen Text erarbeitet und auf ihn bezogen wurden.

Anders als bei Thomsons Vorgehen, bei dem sie die für den Text relevanten Bildelemente vorgibt und damit die Lesart des Bildes aktiv steuert, mussten sich die Studierenden der Weltsprachenuniversität die kompositorischen und inhaltlichen Zusammenhänge von Kunstbild und literarischem Text mittels unterschiedlicher methodischer Ansätze selbst erarbeiten.

Wie Thomson ging ich in meiner Untersuchung davon aus, dass Kunstbild und literarischer Text einander auch ohne werkimmanenten Bezug kriteriengeleitet und dadurch für die Lernenden nachvollziehbar zugeordnet werden können. Gleichzeitig bedeutet dies, dass der rezipientenbestimmte, individuelle Zugang zu Kunstbild und literarischem Text durch Auswahl und Didaktisierung gelenkt und begrenzt wird.

Im Gegensatz zu Thomsons durch die Lehrkraft vordefinierten Bildausschnitten erfolgte die Steuerung in den beiden untersuchten Gruppen an der Weltsprachenuniversität indirekt durch die Bild- und Textaufgaben.

Daraus ergaben sich fünf Variablen, die für den Kunstbildeinsatz im Literaturunterricht DaF in der Untersuchung gesondert betrachtet wurden: (a) das Ziel des Kunstbildeinsatzes im Literaturunterricht (beispielsweise tieferes Textverständnis, Illustration oder Einstimmung), (b) die Kriterien der Zuordnung von Kunstbild und literarischem Text, (c) die methodische Erarbeitung des literarischen Textes, (d) die methodische Erarbeitung des Kunstbildes und (e) die Zusammenführung von Kunstbild und literarischem Text im Unterricht. 


\subsection{Leseprotokolle als Gegenstand der qualitativen Inhalts- analyse}

Um die beiden Forschungsfragen beantworten zu können und sich den Rezeptionsprozessen der literarischen Texte und Kunstbilder durch die Studierenden anzunähern, wurden in beiden Gruppen retrospektive Interviews durchgeführt (vgl. Witzel 2000). Vier der Interviews wurden mittels der zusammenfassenden qualitativen Inhaltsanalyse ausgewertet.

Nach Philipp Mayring $(2000,2001)$ liegen die Vorteile der qualitativen Inhaltsanalyse in ihrer Adaptierbarkeit, der Offenheit der Kategorien sowie der handlichen Abfolge der Analyseschritte. Problematisch erscheinen jedoch die Gewährleistung der Intersubjektivität der empirisch begründeten Kategorien und der aus dem untersuchten Interviewmaterial herausgelösten Ergebnisse sowie die Objektivität der Kategorienbildung.

Im Falle der hier vorgestellten Forschungsarbeit waren zusätzlich zu den Interviews am Beginn des Untersuchungszeitraums Leseprotokolle (vgl. Bredella/ Burwitz-Melzer 2004, Ehlers 1992, Freitag 2010, Hecke 2010, vgl. Abbildung 1) als Hilfsmittel eingeführt worden (vgl. Punkt 2). Ziel war es, Rezeptionsprozesse anzuregen und Gedanken unmittelbar nach dem Lesen des literarischen Textes und Kunstbildeinsatzes festzuhalten.

Während die semi-strukturierten Interviews erst am Ende der Unterrichtseinheiten zu zwei Vergleichstexten (jeweils ein Text mit und ein Text ohne Kunstbildeinsatz) erhoben wurden, fertigten die Studierenden zu jedem im Unterricht behandelten literarischen Text außerhalb des Unterrichts Leseprotokolle an.

Die Studierenden erläuterten anhand ihrer Leseprotokolle am Beginn der Unterrichtssitzungen ihre Gedanken zum gelesenen Text. Ursprünglich lediglich als Erinnerungsstütze für die Studierenden gedacht, erwiesen sich die Leseprotokolle im Verlauf der Untersuchung als Datenquelle, die deutlicher als die retrospektiven Interviews die Veränderungen im Textverständnis sowie die Aufnahme der im Unterricht behandelten Kunstbilder oder daran erarbeiteter Analogien zeigte.

Ziel der in der Forschungsarbeit durchgeführten Triangulation der beiden Datentypen (Interviews und Leseprotokolle) war es, die Rezeptionsprozesse mittels der unterschiedlichen Zugänge zu überprüfen und dadurch die Validität zu erhöhen. Mit der Analyse der Leseprotokolle ergab sich eine weitere, methodische Problemstellung, die mit dem vorliegenden Artikel zur Diskussion gestellt werden soll: Lässt sich die qualitative Inhaltsanalyse nach Philipp Mayring auf den Bereich (semi-)visueller Daten wie Leseprotokolle übertragen? 


\section{Das Leseprotokoll - Definition und Methodik der Daten- erhebung}

\subsection{Definition}

Unter ,Leseprotokoll' wird ein von den Lernenden als Hausaufgabe zu einem literarischen Text gestaltetes, subjektives, mit visuellen oder textlichen Elementen versehenes Dokument verstanden, in dem die Studierenden ihre Gedanken und Fragen zum gelesenen Text darlegen können.

Ursprünglich als rezeptionsästhetisches Mittel von Swantje Ehlers und Lothar Bredella für den Literaturunterricht in Form eines Fragen- und Antwortkatalogs vorgeschlagen (vgl. Bredella/Burwitz-Melzer 2004: 164f., Ehlers 1992: 50ff.), vertrete ich einen weitgefassten Leseprotokollbegriff, der auch visuelle Ausdrucksformen umfasst (vgl. Freitag 2010, Hecke 2010). Leseprotokolle sind jedoch von Textillustrationen zu unterscheiden. Denn während sich Illustrationen auf einzelne Textstellen konzentrieren, werden bei Leseprotokollen Elemente des gesamten Textes integriert, Handlungsstränge dargestellt und kommentiert.

Ziel dieser (semi-)visuellen Auseinandersetzung mit dem Gesamttext ist es, wie bei Bildproduktionen (Illustrationen), die mentalen Modelle der Lernenden zu unterstützen. Denn die Studierenden werden dazu angeregt, in ihren Darstellungen Textinhalte zu konkretisieren und individuelle Vorerfahrungen zu deuten. Durch die vergleichende Präsentation der entstandenen Leseprotokolle im Gruppengespräch können weitere Aushandlungen der Textauslegungen motiviert werden (vgl. Freitag 2010: 183f.). Die zweikanalige Darbietung in Wort und Bild soll dabei die Erinnerung an die Textinhalte verbessern (vgl. Hecke 2010: 169ff.).

\subsection{Methodik der Datenerhebung}

Die Einführung der Leseprotokolle erfolgte in der fünften Unterrichtssitzung mittels eines Gruppenprotokolls, bei dem die Studierenden in Reaktion auf sowohl lehrkraftgesteuerte als auch eigene Fragen an den literarischen Text Gedanken auf einem Blatt im Format DIN A1 fixieren konnten. Die Instruktionssprache war Deutsch. Allerdings wurden weder Vorgaben zur Sprache noch zur Form der Darstellung gemacht.

Auf dem gemeinsamen Gruppenprotokoll waren unterschiedliche Modi beispielhaft dargestellt worden. Es wurde betont, dass Sprache und Form durch die Lernenden selbst gewählt werden. Die Aufzeichnung der Gedanken war somit in der Muttersprache (russisch oder usbekisch), auf Deutsch, ohne Verschriftlichung durch rein visuelle Elemente, tabellarisch, schematisch oder als Collage möglich (vgl. Abbildungen 1-4). 
Die Studierenden erhielten am Ende der Instruktionssitzung einen Fragenkatalog mit Impulsfragen (vgl. Tabelle 1), den sie für die Erstellung der Leseprotokolle nutzen konnten. Der Fragenkatalog greift die Anregungen von Ehlers sowie von Bredella und Burwitz-Melzer auf (vgl. Bredella/Burwitz-Melzer 2004: 164f., Ehlers 1992: 50ff.), lässt jedoch grafisch-visuelle Umsetzungen zu.

Tabelle 1: Orientierungsfragen für die Erstellung der Leseprotokolle (nach Bredella/BurwitzMelzer 2004: 164f., Ehlers 1992: 50ff.)

\begin{tabular}{|l|l|}
\hline \multicolumn{2}{|c|}{ Möglicher Fragenkatalog für das Leseprotokoll: } \\
\hline Wer ist der Autor? & Konnte ich die Geschichte leicht und flüssig \\
Was will ich wissen? & lesen? \\
Was macht mich neugierig? & Was ist leicht an dem Text? \\
Was ist besonders an diesem Text? & Was ist schwer an dem Text? \\
Wie viele Personen gibt es im Text? & Was verstehe ich nicht? \\
Was erfahre ich über die Personen? & Was fällt mir an der Sprache? \\
Was fällt mir an den Personen auf? & Wie lautet das Thema? \\
Mag ich die Personen? & Was fällt mir an dem Thema auf? \\
Wer ist der Erzähler? & Hat mich das Ende überrascht? \\
Wie ist der Text aufgebaut? & Was ist fremd für mich an diesem Thema? \\
Was ist fremd für mich am Verhalten der & Warum gefällt mir diese Geschichte? \\
Personen? & Warum gefällt mir diese Geschichte nicht? \\
\hline
\end{tabular}

Sie sollten als Hausaufgabe ein eigenes Leseprotokoll zum zuvor gelesenen Text „Die Küchenuhr“ von Wolfgang Borchert, der die Grundlage für das gemeinsame Leseprotokoll darstellte, anfertigen. Es fiel auf, dass sich die Studierenden in beiden Gruppen vom Fragenkatalog entfernten und sich ihr Stil zunehmend individualisierte. Die Mehrzahl der Studierenden verwendete eine Mischung aus visuellen und textlichen Elementen, die sie mit Kommentaren versahen (vgl. Abbildungen 1-4). Hervorzuheben ist, dass der Kunstcharakter der Darstellungen weder in der Unterrichtsbesprechung noch in der Auswertung eine Rolle spielte und die Studierenden aufgrund der selbstbestimmten Darstellungsmodi anfängliche Unsicherheiten diesbezüglich ablegten.

Bis zu den in der Forschungsarbeit ausgewerteten Unterrichtsitzungen (20. bis 25. Doppelstunde) zu Peter Bichsels Kurzgeschichte „San Salvador“ und Kurt Martis Text „Neapel sehen“ hatten die Studierenden ihren eigenen Stil entwickelt, der nicht mehr durch das gemeinsame Leseprotokoll beeinflusst war. Die Datensammlung zur untersuchten Unterrichtsreihe umfasst insgesamt 43 Leseprotokolle. 


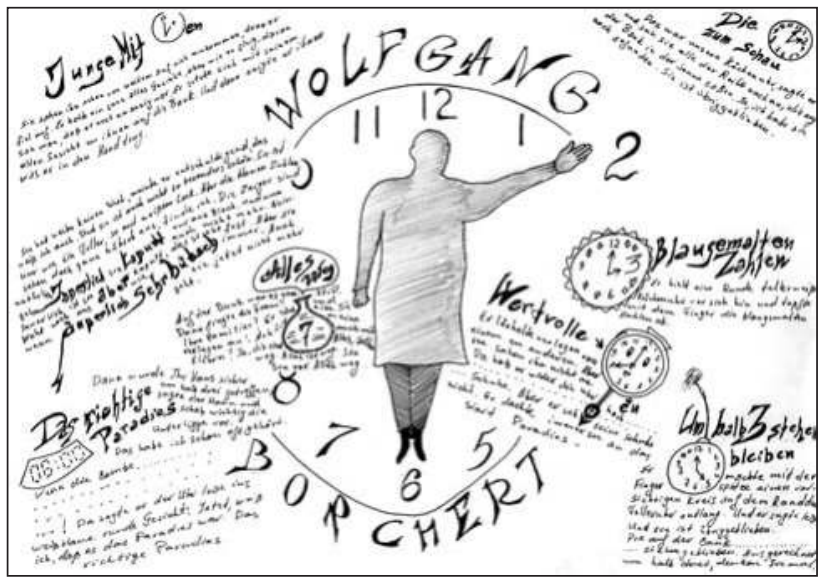

Abbildung 1: Erstes Leseprotokoll eines Studenten zu Borcherts Text „Die Küchenuhr“

Am Beginn jeder Unterrichtsstunde erhielten die Studierenden Zeit, ihre Leseprotokolle vorzustellen und anhand ihrer Darstellungen ihre Ideen zu rekapitulieren. Anders als die problemorientierten, semi-strukturierten Interviews, in denen die Befragten nach sechs Doppelstunden über die beiden mit oder ohne Kunstbild behandelten Texte Bichsels und Martis reflektierten, boten die Leseprotokolle ein direkteres, von Zeit- und Kommunikationsdruck unbeeinflusstes Verfahren, anhand dessen der weitere Unterricht gestaltet wurde.

Die auf der Basis aller entstandenen Leseprotokolle abgeleiteten Analysekategorien wurden zunächst anhand der mündlichen Explikationen der Studierenden zu ihren Protokollen im Unterricht geprüft (Audioaufnahmen im Mp3Format), bevor die Leseprotokolle der untersuchten Unterrichtseinheiten mittels der zusammenfassenden qualitativen Inhaltsanalyse nach Philipp Mayring ausgewertet wurden.

\section{Analyse der Leseprotokolle}

\subsection{Grundlagen des Auswertungsverfahrens}

Die Grundlage für das Auswertungsverfahren der visuell oder textlich gestalteten Leseprotokolle bildete eine Untersuchung Peter Kuhns, der - angelehnt an die Prinzipien der qualitativen Inhaltsanalyse Philipp Mayrings $(2000,2001)$ - problemzentrierte Kinderzeichnungen kategorisiert, zusammenfasst und analysiert (vgl. Kuhn 2003: Absatz 30). Für den Sportpsychologen Kuhn bildeten die Zeichnungen den Ausgangspunkt für problemzentrierte Interviews, in denen die Kinder ihre Zeichnungen erläuterten. Mein Vorgehen wich insofern von dem Kuhns ab, als dass die Leseprotokolle nicht als Grundlage für ein Interview mit 
den Probanden dienten. Die Leseprotokoll- und Interviewdaten wurden unabhängig voneinander erhoben, waren aber durch ihren Inhalt miteinander verbunden.

Wie Kuhn orientierte ich mich an der qualitativen Inhaltsanalyse Mayrings. Sie bot für die Fragestellung der Untersuchung den Vorteil, durch die zyklisch angelegten, leicht adaptierbaren Arbeitsschritte sowohl den offenen Charakter der Untersuchung als auch die theoretische Auseinandersetzung mit der Fachliteratur berücksichtigen zu können. Gleichwohl musste das durch Mayring explizierte Textanalyseverfahren an die (semi-)visuellen Leseprotokolle angepasst werden.

Die Leseprotokolle wurden inhaltlich zusammengefasst und darauf untersucht,

- wie die im Unterricht behandelten Texte subjektiv verarbeitet (vgl. Hecke 2010: 170) und

- inwiefern die im Unterricht behandelten Kunstbilder in die Gestaltung der Leseprotokolle aufgenommen wurden.

Wie in Kuhns Herangehensweise standen die Erfassung subjektiver Texteindrücke und -auslegungen respektive die Aufnahme von Unterrichtsinhalten und Kunstbildelementen im Mittelpunkt der Betrachtung. Die Inhalte der Leseprotokolle wurden identifiziert und auf der Grundlage der literarischen Texte, der behandelten Kunstbilder sowie der als Mp3-Dateien aufgenommen Unterrichtssequenzen interpretiert.

\subsection{Auswertungsverfahren}

Peter Kuhn (2003) entwickelt für die Analyse der thematischen Kinderzeichnungen ein Vier-Schritte-Verfahren, das sich aus (1) der deskriptiven Identifikation der Bildelemente, (2) der interpretativen Erschließung von Kategorien auf Grundlage der Bildelemente und (3) der interpretativen Zuordnung von Bildern zu Bildmotiven auf einer höheren Abstraktionsebene mit dem Ziel der (4) Erstellung von Deskripten als verbale Zusammenfassung der visuellen Inhalte zusammensetzt.

Kuhns Vorgehen diente mir als Vorlage, um die qualitative Inhaltsanalyse Mayrings auf (semi-)visuelle Daten zu übertragen. Da die Leseprotokolle jedoch anders als bei Kuhns Kinderzeichnungen, in denen die Kinder ihre Vorstellung von ihrem Traumspielplatz darstellen sollten, in direkter Reaktion auf einen gelesenen literarischen Text und die Unterrichtsgestaltung entstanden waren, wurden seine vier Analyseschritte erweitert und stärker an Mayrings Verfahren angelehnt. 
Tabelle 2: Ankerbeispiele für die deskriptive Identifikation der Leseprotokollelemente

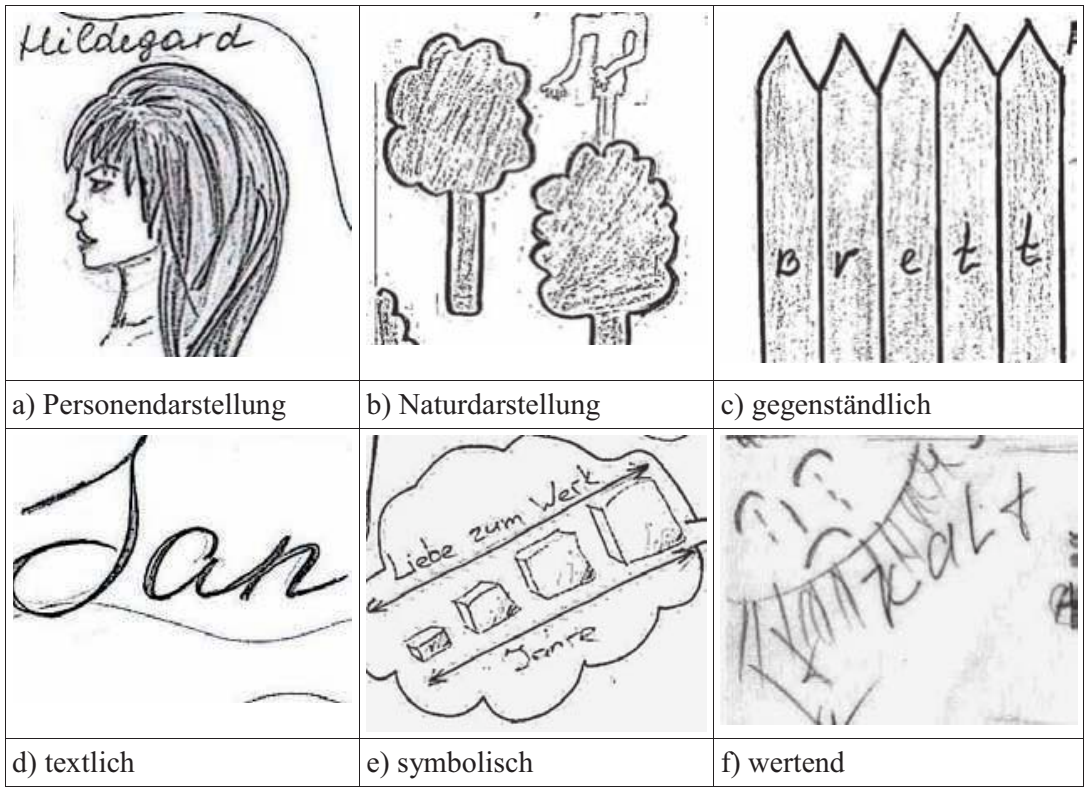

1. Schritt: Identifikation der Leseprotokollelemente: Die Leseprotokolle wurden anhand ihrer Elemente in Bild- oder Textform klassifiziert. Dazu wurde bei der Identifikation eine Unterteilung in (a) Personendarstellung, (b) Naturdarstellung (Feuer, Wolke, Palme), (c) gegenständliche (Haus), (d) textliche (Titel, Beschriftung, Sprechblase), (e) symbolische (Pfeile, Satzzeichen) und (f) wertende Elemente (Smileys) vorgenommen (vgl. Tabelle 2).

2. Schritt: Komposition und Leserichtung: Obwohl Bildwerke in ihrer Totalität erfassbar sind, geben BildproduzentInnen mittels Komposition und Räumlichkeit Hinweise darauf, wie die Darstellung gelesen werden muss. Leseprotokolle vereinen aufgrund ihrer visuellen und textlichen Elemente sowohl die vielseitige bildlich-kompositorische Komplexität als auch die textliche Linearität. Unter den analysierten Leseprotokollen ließen sich sechs Leserichtungen identifizieren: (a) zentral/ zirkulär (von einem Punkt oder einer Kreisbewegung ausgehend), (b) dezentral mittels verschiedener Streuelemente, (c) polarisierend als Gegenüberstellung von zwei Seiten, (d) linear-horizontal, (e) linear-vertikal und (f) schematisch (vgl. Tabelle 3).

3. Schritt: Identifikation von Text- und Bildzitaten: Text- und Bildzitate lassen auf die intensive Auseinandersetzung mit dem Ausgangsmaterial schließen. Die Aufnahme von Elementen der im Unterricht behandelten Bilder verweist auf den Transfer bildlicher Inhalte auf die Textebene. Allerdings schließt dies mögliche 
Interferenzen einer fehlerhaften Bildinterpretation sowie einer einseitigen Textrezeption ein. Bei der Analyse der untersuchten Leseprotokolle wurde zwischen (a) einem Textzitat (ein Auszug des literarischen Textes), (b) einem unterrichtsinternen Bildzitat (eine Übernahme von im Unterricht behandelten Kunstbildern oder Elemente von diesen) und (c) einem unterrichtsexternen Bildzitat (eine Verknüpfung des literarischen Textes mit Bildern, die nicht im Unterricht behandelt wurden) unterschieden (vgl. Tabelle 4).

Tabelle 3: Auszug aus den Ankerbeispielen für Kompositionsvarianten

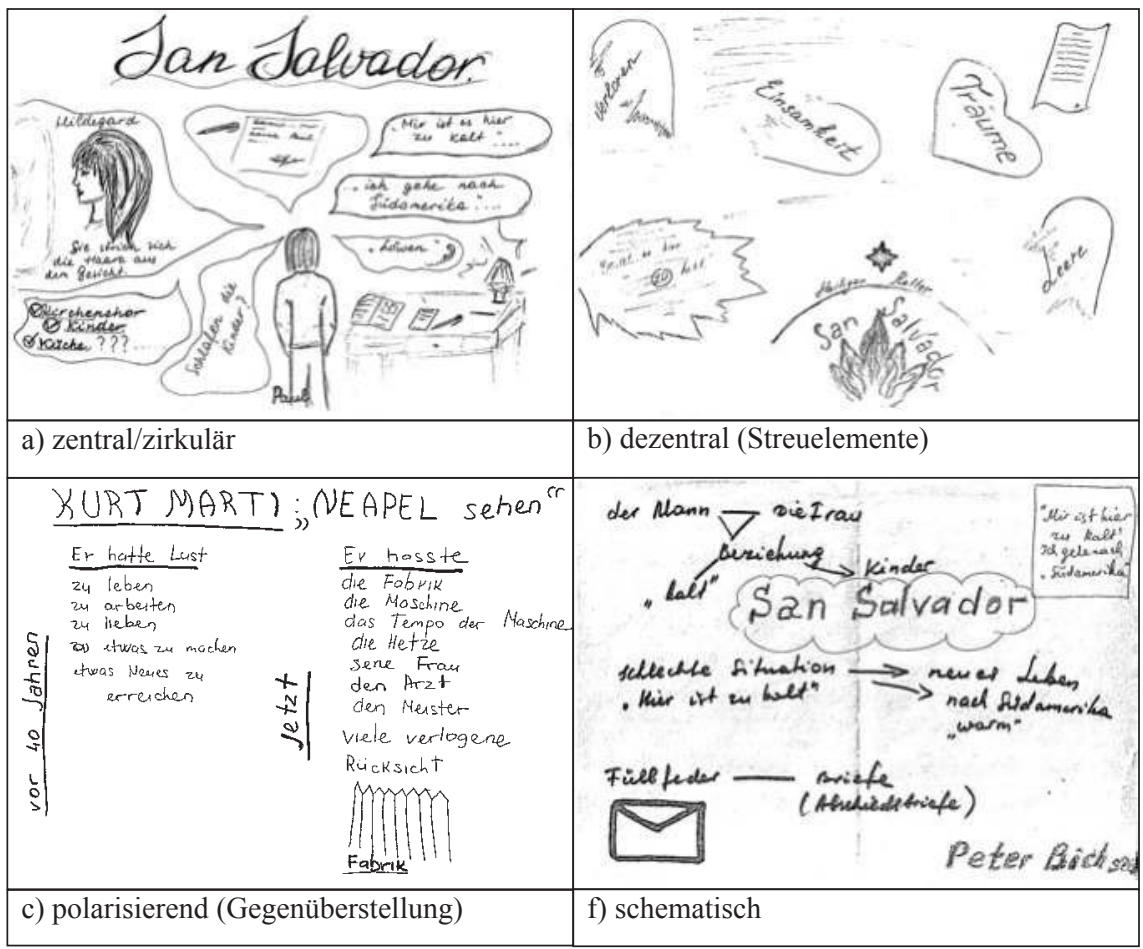

4. Schritt: Paraphrasierung der Leseprotokollelemente: Auf Basis der Identifikation von visuellen und textlichen Elementen wurden die Inhalte der Leseprotokolle zu einem Raster, das heißt einer Auflistung von Elementen der Leseprotokolle, zusammengestellt.

5. Schritt: Generalisierung (Abstraktionsebene zur Klassifizierung des Inhalts): Die im Leseprotokoll identifizierten visuellen oder textlichen Elemente wurden klassifiziert und auf die zu untersuchenden Aspekte (Verarbeitung der literarischen Texte, Aufnahme der behandelten Kunstbilder) bezogen. 
Tabelle 4: Ankerbeispiele für Text- und Bildzitate

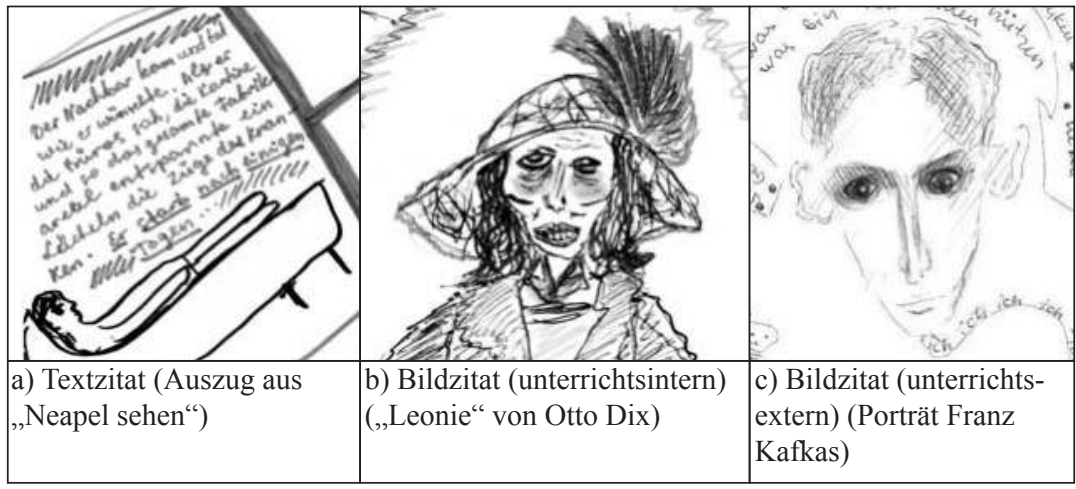

6. Schritt: Kategorienbildung: Von den Generalisierungen wurden Kategorien abgeleitet und ein Kodierleitfaden (vgl. Tabelle 5) erstellt. Die als Mp3-Dateien aufgenommenen Erläuterungen der Studierenden im Unterricht dienten dabei der Absicherung der gebildeten Kategorien. Nach einer weiteren Überprüfung der Leseprotokolle und der ermittelten Kategorien wurden zwölf Leseprotokolle von vier im Vorfeld durch ein Datensampling ermittelten Testpersonen (je zwei Personen aus beiden Studiengruppen) auf der Grundlage des Kodierleitfadens ausgewertet. Der Kodierleitfaden verweist in der ersten Spalte auf die Leseprotokolle (LP), die die Ankerbeispiele für die Kategorien bilden, und gibt in den nachfolgenden Spalten das im jeweiligen Ankerbeispiel relevante Element als Paraphrase sowie die Definition der Kategorie und die Kategorienbezeichnung an.

Tabelle 5: Auszug aus dem Kodierleitfaden für die Auswertung der Leseprotokolle

\begin{tabular}{|l|l|l|l|c|}
\hline Ankerbeispiel & Paraphrasierung & Generalisierung & \multicolumn{1}{c|}{ Definition } & Kategorie \\
\hline $\begin{array}{l}\text { LP 7 (KI-A zu } \\
\text { „Neapel sehen“) }\end{array}$ & $\begin{array}{l}\text { Bäumchen (Zitat) } \\
\text { als Symbol der } \\
\text { Innenwelt, Fabrik } \\
\text { als Außenwelt }\end{array}$ & $\begin{array}{l}\text { Aufnahme eines } \\
\text { unterrichts- } \\
\text { internen Bild- } \\
\text { elementes }\end{array}$ & $\begin{array}{l}\text { Auslegung beruft } \\
\text { sich auf Kunstbild- } \\
\text { zitate oder Unter- } \\
\text { richtselemente }\end{array}$ & $\begin{array}{l}\text { durch Unter- } \\
\text { richtsinhalte } \\
\text { erweitert }\end{array}$ \\
\hline
\end{tabular}

Insgesamt wurden für die 43 Leseprotokolle sechs Kategorien ermittelt: (a) inhaltlich-zusammenfassend (hochgradig textbezogen), (b) fragend-kommentierend (Textreflexion), (c) nicht im Text identifizierbar subjektiv erweitert (Fortsetzungen und Interferenzen), (d) monoperspektivisch (subjektiver Fokus), (e) durch Unterrichtsinhalte erweitert (Bild- und Textzitate) und (f) multiperspektivisch (mehrere Auslegungen). Unter der Kategorie ,inhaltlich-zusammenfassend" wurde ein Leseprotokoll verstanden, das ausschließlich den Inhalt des Textes wiedergibt und keine eigenen Wertungen oder Fragen enthält. Die Kategorie ,fragend-kommentierend“ bezeichnet die dialogische Auseinandersetzung 
mit dem Text. In derartigen Leseprotokollen wurden Fragen und Kommentare zum Inhalt, dessen Wirkung und Bedeutung gestellt. Enthielt das Leseprotokoll Angaben, die sich nicht am Text belegen lassen, wurden diese als ,nicht im Text identifizierbare subjektive Erweiterungen' zusammengefasst. Diese Kategorie umfasst zum einen durch den Text ausgelöste, jedoch nicht mehr an diesen gebundene Fortsetzungen und zum anderen Interferenzen (vgl. Ehlers 2007: 121f.), die auf Textschwierigkeiten und Missverständnisse hinweisen. Davon zu unterscheiden sind sogenannte, monoperspektivische“ Darstellungen, die sich auf einen herausgelösten Aspekt des gelesenen Textes konzentrieren und andere Auslegungen vernachlässigen. Wurden dagegen Kunstbildzitate oder im Unterricht thematisierte Inhalte in ein Leseprotokoll aufgenommen, klassifizierte ich diese als ,durch Unterrichtsinhalte motivierte Erweiterungen'. Setzt sich der Ersteller mit mehreren Auslegungen auseinander und stellt diese im Leseprotokoll dar, wurde das Leseprotokoll als ,multiperspektivisch ${ }^{\text {}}$ kategorisiert.7. Schritt: Erstellung von Deskripten: Auf der Grundlage der vorangestellten sechs Analyseschritte wurden die zwölf Leseprotokolle der vier Testpersonen zu Deskripten, das heißt tabellarischen Inhaltsbeschreibungen, zusammengefasst (vgl. Tabelle 6). Die Deskripte enthalten zum einen Angaben zum Entstehungskontext (,Bezugsrahmen') sowie inhaltlich-kompositorische Merkmale und zum anderen jene Kategorien, nach denen das Leseprotokoll anhand des Kodierleitfadens kodiert wird. In den Deskripten der einzelnen Leseprotokolle wurden alle enthaltenen Kategorien angeführt, um die sich anschließende Interpretation nicht auf eine Kategorie zu reduzieren.

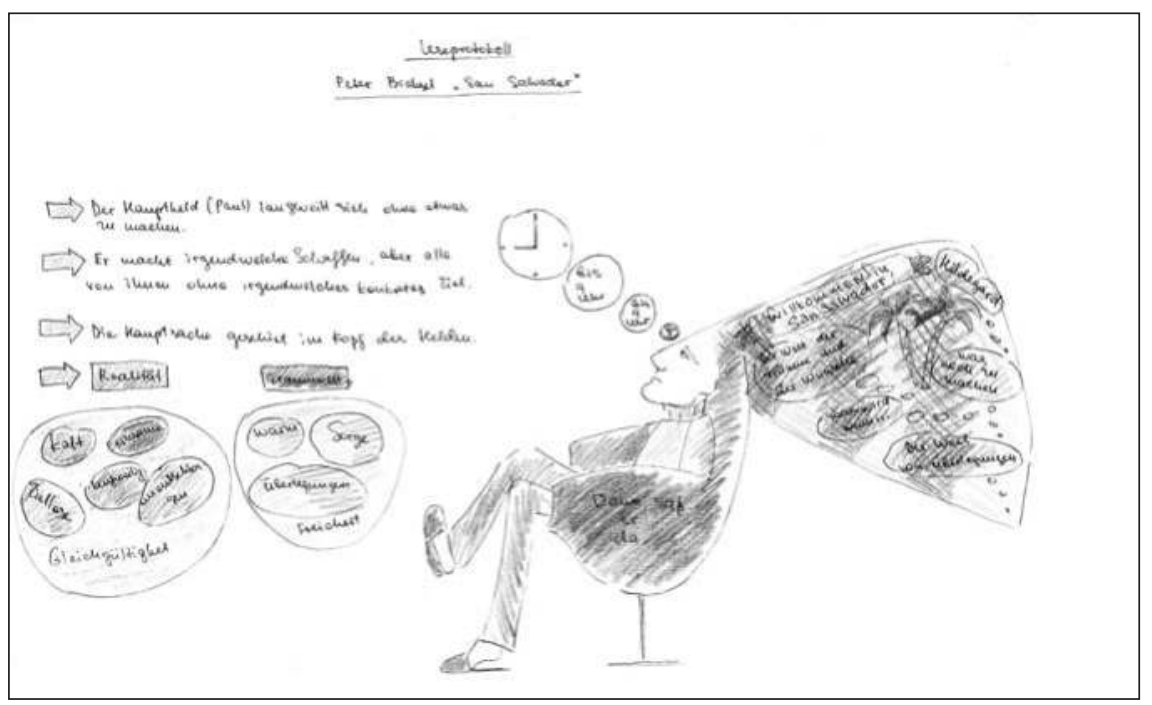

Abbildung 2: Leseprotokoll (LP 14) von KI-A zu Peter Bichsels „San Salvador“ 
Tabelle 6: Auszug aus dem zusammenfassenden Deskript der Leseprotokolle der Probandin KI-A zu Abbildung 2 (LP 14)

\begin{tabular}{|l|l|}
\hline \multicolumn{1}{|c|}{ LP 14 (KI-A) } & \multicolumn{1}{|c|}{ Kriterium } \\
\hline „San Salvador“, nach der unterrichtlichen Textarbeit in Gruppen & Bezugsrahmen \\
\hline Querformat, schematische Zeichnung mit Textbereich & Format/ Gattung \\
\hline $\begin{array}{l}\text { Pfeile weisen auf textliche Zusammenfassung hin: Paul langweilt sich, in } \\
\text { zwei Blasen textlich-kommentierende Begriffe zu Realität und Traumwelt } \\
\text { zusammengestellt, Kopf der sitzenden Figur mit Gedankenblasen }\end{array}$ & $\begin{array}{l}\text { inhaltliche } \\
\text { Elemente }\end{array}$ \\
\hline linear-horizontal & Komposition \\
\hline im Stuhl: „Dann saß er da.“(Text „San Salvador“) & Bild-/Textzitat \\
\hline monoperspektivisch, kommentierend & Kategorien \\
\hline
\end{tabular}

8. Schritt: Vergleich und Interpretation - Am Fallbeispiel KI-A: Die Deskripte bildeten den Ausgangspunkt sowohl für den Vergleich der in den Leseprotokollen enthaltenen Hinweise auf das Textverständnis der vier Testpersonen als auch für die Triangulation der Leseprotokoll- und Interviewdaten, wie am Fallbeispiel KI-A (vgl. Abbildungen 2-4) illustriert. Die Probandin KI-A hatte sich, wie die Analyse ihrer drei Leseprotokolle ergab, intensiv mit den literarischen Texten und eingesetzten Kunstbildern beschäftigt. Ihre Leseprotokolle waren stark strukturiert, was meines Erachtens darauf hinweist, dass ihre Leseprotokolle nicht parallel zum Lesen als rein subjektiver Akt entstanden, sondern als Resultat einer eingehenden Textreflexion.

Nach dem ersten Lesen des Textes Kurt Martis „Neapel sehen“ und der bildbeschreibenden Auseinandersetzung mit den beiden durch mich zugeordneten Kunstbildern Gustav Wunderwalds „Fabrik Loewe \& Co. (Moabit)“ (vor 1929, Öl auf Leinwand, keine Größenangabe, Berlinische Galerie) und Oswald Achenbachs „Küstenlandschaft bei Neapel“ (1882, Öl auf Leinwand, 139 x $195 \mathrm{~cm}$, Verbleib unbekannt) wählte KI-A eine künstlerisch-bildproduktive Auseinandersetzung in Form einer Collage (Abbildung 3), in der sie verschiedene Papiersorten, Scherenschnitte und Zeichnungen mischte. Im zweiten Leseprotokoll zu Martis Text sowie der Darstellung des Vergleichstextes Peter Bichsels „San Salvador" ging sie zu schematischen Zeichnungen über (Abbildung 2 und 4).

In ihrem ersten, hochformatigen, linear komponierten Leseprotokoll (Abbildung 3) zu Kurt Martis Text dominiert das Motiv der Fabrik, dem sie eine Zeichnung eines Mannes mit Haus und Garten gegenüberstellt. Ihr monoperspektivischer Fokus liegt auf der Trennung der Welt des Mannes von der einer übermächtigen Fabrik. Die Darstellung des Fabrikgebäudes, durch weißes Papier vom braunen Untergrund abgesetzt, wird durch Fenster und Schriftzüge, die Begriffe aus dem Text aufgreifen, strukturiert. Die Verlängerung der Fabrik in den oberen Bildteil 
ist als Bretterwand zu verstehen, die den Lebensbereich der Hauptfigur im Text von der benachbarten Fabrik trennt.

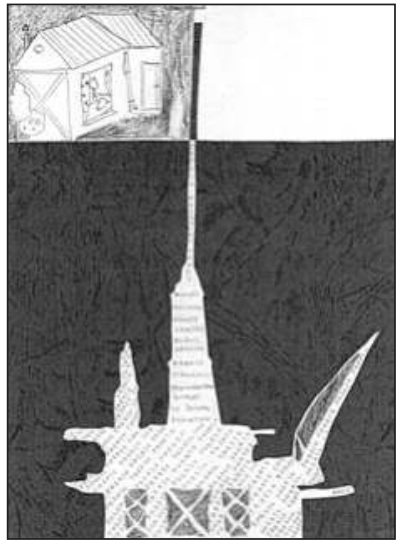

Abbildung 3: Erstes Leseprotokoll (LP 6) von KI-A zu Kurt Martis „Neapel sehen“

Nach einer Stationenarbeit, bei der die beiden dem Text zugeordneten Kunstbilder Wunderwalds und Achenbachs im Unterricht verglichen, analysiert und auf den Text bezogen wurden, veränderte sich der Fokus von KI-A, wie ihr zweites Leseprotokoll zeigt (Abbildung 4). Das zweite Leseprotokoll von KI-A zu Martis Text ist im Querformat als schematische Zeichnung mit Textkommentaren angelegt. In der linear-horizontalen, analytischen Komposition stellt KI-A einen vertrockneten (Ausschnitt aus Wunderwalds Gemälde) und einen grünen Baum (Ausschnitt aus Achenbachs Bild), einen Mann, in dessen Innerem ein Baum grünt, eine Fabrik und eine Bretterwand zeichnerisch dar und erläutert die Elemente in den Textfeldern. Die Bäume dienen KI-A, wie sie im Textfeld darlegt, als Symbol für die innere Welt des Mannes (vgl. Personendarstellung), die sich am Ende des Marti-Textes der Außenwelt öffnet, indem der Mann die den Garten (im Text durch das Diminutiv „Gärtchen“ sprachlich hervorgehoben) isolierende Bretterwand abreißen lässt und lächelnd stirbt. Der Zusammenhang von Garten („Gärtchen“) und innerer Welt sowie der Öffnung nach außen war im Unterricht von der Gruppe an Text und Kunstbildern erarbeitet worden. Wenngleich die Probandin KI-A ihr Augenmerk auf diesen Aspekt beschränkt, spricht die argumentative Aufnahme der Bildzitate in das zweite Leseprotokoll für ihre Auseinandersetzung mit den Kunstbildern, die sie als Analogie für den Marti-Text benutzt. 


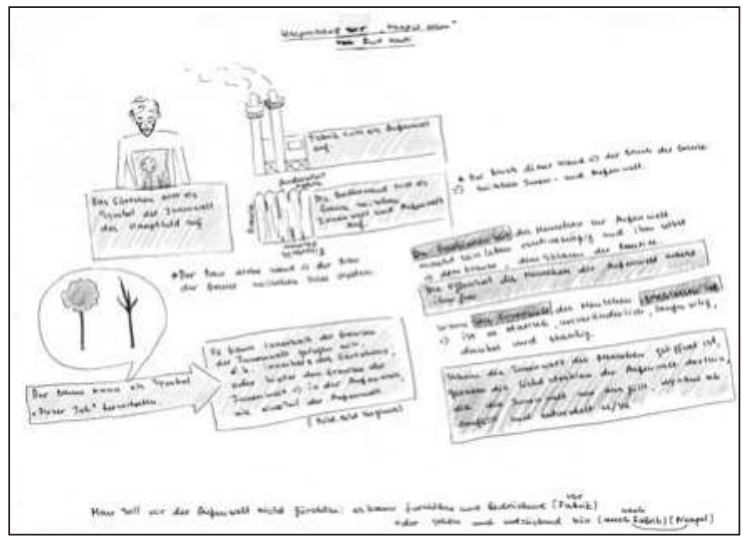

Abbildung 4: Zweites Leseprotokoll (LP 7) von KI-A zu Kurt Martis „Neapel sehen“

Im Interview konnte sich KI-A trotz des zeitlichen Abstandes zwischen Interview und Unterrichtseinheiten (drei Doppelstunden) reflektiert zu unterschiedlichen Textauslegungen zu Kurt Martis „Neapel sehen“ sowohl äußern als auch positionieren. Die Triangulation der Leseprotokoll- und Interviewdaten ergab, dass beides auf eine intensive Auseinandersetzung mit den im Unterricht eingesetzten Kunstbildern und dem literarischen Text zurückzuführen war und ein ,tieferes Textverständnis' im Sinne der eingangs dargelegten Definition vorlag. Im Gegensatz dazu äußerte sich KI-A im Interview wenig konkret zum Text Peter Bichsels, der ohne Kunstbild behandelt worden war. Zwar war KI-A in der Lage, die beiden Texte zu verbinden, aber weder im Leseprotokoll (Abbildung 2) noch im Interview, das unmittelbar im Anschluss an die Unterrichtssitzungen durchgeführt wurde, ließ sich ein ,tieferes Textverständnis' nachweisen.

\section{Der Einsatz von Leseprotokollen im Literaturunterricht}

Leseprotokolle ermöglichen die Annäherung an die Rezeptionsprozesse und bieten einen direkten, subjektiven Einblick in die Verarbeitung des gelesenen Textes und seiner Behandlung im Unterricht, sofern ihr Einsatz kontinuierlich trainiert und Sprache sowie Form der Darstellung durch die Lernenden bestimmt werden. Anders als Interviews unterliegen Leseprotokolle, wie in der vorliegenden Studie eingesetzt, somit nicht der Gefahr der direkten oder indirekten Steuerung durch den Interviewer. Allerdings erlaubt ihre Erstellung außerhalb des Unterrichts keine unmittelbaren Nachfragen zu den auf dem Papier festgehaltenen Reflexionen und Darstellungsweisen. Dies ist nur im Rahmen einer (in diesem Fall zeitversetzten) Erläuterung der Leseprotokolle möglich. 
Es erwies sich als vorteilhaft, jeweils nach dem ersten Lesen des Textes und nach der gemeinsamen Textbesprechung im Unterricht (dem zweiten Lesen) ein Leseprotokoll anfertigen zu lassen. Die Lernenden wurden dadurch zur nochmaligen Auseinandersetzung mit dem Text und der Erstkonzeption ihres Leseprotokolls angehalten.

\section{$5 \quad$ Fazit - Die Auswertung (semi-)visueller Daten durch die qualitative Inhaltsanalyse nach Philipp Mayring}

Für die untersuchte Fragestellung wurde die zusammenfassende qualitative Inhaltsanalyse Philipp Mayrings auf (semi-) visuelle Leseprotokolle übertragen. Die Auswertung der Leseprotokolle ging von den Annahmen aus, dass sich

(1)Rezeptionsprozesse anhand ihrer Visualisierung rekonstruieren und

(2) individuelle Darstellungsmodi zusammenfassen und kodifizieren lassen.

Das Schrittverfahren der qualitativen Inhaltsanalyse Mayrings und die Offenheit ihrer Kategorienbildung ermöglichen eine leicht zu realisierende Adaption und Auswertung der erhobenen Interview- und Leseprotokolldaten, deren Triangulation durch die Verwendung des gleichen Analyseverfahrens vereinfacht wurde.

Gleichzeitig erforderte die Anpassung der Vorgehensweise Mayrings an visuelle Daten,

- die Berücksichtigung ihrer medialen Besonderheiten;

- ihrer Verbalisierung;

- die Einschränkung ihrer scheinbaren ,Deutungsoffenheit";

- die Reduktion/Generalisierung individueller, sprachlich und formal nicht vordefinierter Darstellungsmodi;

- den Einbezug individuell und kulturell geprägter Bilderfahrungen;

- sowie die Bildung intersubjektiv nachvollziehbarer Kategorien.

Die medialen Besonderheiten können meines Erachtens durch die Analyseschritte 1 bis 3, in denen alle Leseprotokollelemente, die Komposition, die Leserichtung sowie die Text- und Bildzitate identifiziert werden, eingebunden werden. Schwieriger gestaltet sich dagegen die Überführung in das Medium Text und die Paraphrasierung sowie Generalisierung (Analyseschritte 4 und 5) der identifizierten Elemente. Durch die Verbalisierung der Leseprotokolle wird zwar deren Vergleichbarkeit hergestellt, die Intersubjektivität der beschreibenden Formulierungen lässt sich jedoch nur durch den Gebrauch festgelegter Termini absichern, mit denen originär die Kunst- und Bildwissenschaften operieren. Da sich Leseprotokolle zusätzlich auf einen literarischen Text beziehen, bedarf es für die Generalisierung und Kategorienbildung transdisziplinärer An- 
sätze, um ein geeignetes terminologisches Instrumentarium zu entwickeln. In jedem Fall müssen die Abbildungen der Leseprotokolle der Auswertung beigefügt werden.

Im Gegensatz $\mathrm{zu}$ freien Visualisierungen wird die scheinbare ,Deutungsoffenheit' bildlicher Darstellungen durch die enge Anbindung der Leseprotokolle an den literarischen Text sowie an die im Unterricht eingesetzten Kunstbilder eingeschränkt. Dieser Bezug vereinfacht die Analyse, da individuelle Auslegungen und Interferenzen unmittelbar identifiziert werden können. Problematisch erscheint dabei jedoch die Frage nach den individuell und kulturell geprägten Text- und Bildtraditionen der Lernenden. Diese beeinflusst die Reflexion und ggf. Darstellungsweise und muss daher bei der Auswertung Berücksichtigung finden bzw. ausgeschlossen werden. Im untersuchten Fall konnte dieser Aspekt aufgrund der reduzierten Bildvorerfahrungen der Studierenden und der systematisch während des Untersuchungszeitraums durchgeführten Sehschulung ausgeklammert werden. Dennoch müssen sich weiterführende Studien eingehender mit Interferenzen durch Bildvorerfahrungen sowohl auf Seite der Lernenden als auch auf Seite des Auswerters befassen.

Die Kategorienbildung ist der entscheidendste und problematischste Analyseschritt. Sie erfolgt mittels der Identifikation der Leseprotokollelemente, ihrer Paraphrasierung und ihrer Generalisierung. Der in diesem Schritt entstandene Kodierleitfaden, der die einzelnen Analyseschritte zusammenfasst, dient als Grundlage für die weitere Auswertung. Allerdings stellt sich erneut die Frage nach seiner Transparenz und intersubjektiven Nachvollziehbarkeit.

Im Forschungsprojekt an der Weltsprachenuniversität Taschkent wurden zusätzlich zur Analyse der (semi-)visuellen Daten die Erläuterungen der Leseprotokolle durch die Studierenden im Unterricht elektronisch aufgezeichnet. Die Erklärungen der Studierenden stützten die anhand der Leseprotokollanalyse abgeleiteten Kategorien. Es empfiehlt sich in Untersuchungen zu Einsatz und Auswertung von Leseprotokollen, stärker zwischen der Kategorienbildung auf der Grundlage der visuellen Datenanalyse und den retrospektiven Explikationen im Unterrichtskontext zu differenzieren.

Als Fazit lässt sich festhalten, dass sich das Auswertungsverfahren Mayrings in adaptierter Form auf (semi-)visuelle Leseprotokolle anwenden lässt, jedoch weiter untersucht werden muss. 


\section{Literatur}

Bach, Gerhard / Breidbach, Stephan (2009). Fremdsprachenkompetenz in der mehrsprachigen Wissensgesellschaft. In: Bach, Gerhard / Timm, Johannes-Peter (Hrsg.): Englischunterricht. Tübingen / Basel: Francke. 280-303.

Boehm, Gottfried (2001). Die Wiederkehr der Bilder. In: Boehm, Gottfried (Hrsg.): Was ist ein Bild? München: Fink, 3. Aufl. 11-38.

Bredella, Lothar / Burwitz-Melzer, Eva (2004). Rezeptionsästhetische Literaturdidaktik mit Beispielen aus dem Fremdsprachenunterricht Englisch. Tübingen: Narr.

Ehlers, Swantje (1992). Lesen als Verstehen. Kassel u. a.: Langenscheidt. (Fernstudieneinheit 2)

Ehlers, Swantje (2007). Lesetheorien, Lesekompetenz und Narrative. In: Bredella, Lothar / Hallet, Wolfgang (Hrsg.): Literaturunterricht, Kompetenzen und Bildung. Trier: Wissenschaftlicher Verlag. 107-126.

Freitag, Britta (2010). Visualisierungsaufgaben im Literaturunterricht: Lernerorientierte Zugangsweisen zu Andrea Levys Roman Small Island in Schule und Hochschule. In: Hecke, Carola / Surkamp, Carola (Hrsg.): Bilder im Fremdsprachenunterricht: Neue Ansätze, Kompetenzen und Methoden. Tübingen: Narr. 181-198.

Hallet, Wolfgang (2010). Viewing cultures: Kulturelles Sehen und Bildverstehen im Fremdsprachenunterricht. In: Hecke, Carola / Surkamp, Carola (Hrsg.): Bilder im Fremdsprachenunterricht: Neue Ansätze, Kompetenzen und Methoden. Tübingen: Narr. 26-54.

Hecke, Carola (2010). Zum Gewinn bildproduktiven Arbeitens im fremdsprachlichen Literaturunterricht. In: Hecke, Carola / Surkamp, Carola (Hrsg.): Bilder im Fremdsprachenunterricht: Neue Ansätze, Kompetenzen und Methoden. Tübingen: Narr. 165-180.

Hecke, Carola / Surkamp, Carola (2010). Einleitung: Zur Theorie und Geschichte des Bildeinsatzes im Fremdsprachenunterricht. In: Hecke, Carola / Surkamp, Carola (Hrsg.): Bilder im Fremdsprachenunterricht: Neue Ansätze, Kompetenzen und Methoden. Tübingen: Narr. 9-24.

Kuhn, Peter (2003). Thematische Zeichnung und fokussiertes, episodisches Interview am Bild: Ein qualitatives Verfahren zur Annäherung an die Kindersicht auf Bewegung, Spiel und Sport in der Schule. Forum: Qualitative Sozialforschung 4/1, Art. 8. Online: http://www.qualitativeresearch.net/index.php/fqs/article/view/750/1625. (letzter Aufruf [13.03.2011]).

Legutke, Michael K. (2009). Lernertexte im handlungsorientierten Fremdsprachenunterricht. In: Abendroth-Timmer, Dagmar / Elsner, Daniela / Lütge, Christiane / Viebrock, Britta (Hrsg.): Handlungsorientierung im Fokus: Impulse und Perspektiven für den Fremdsprachenunterricht im 21. Jahrhundert. Frankfurt a. M. u. a.: Lang. 203-216.

Mayring, Philipp (2000). Qualitative Inhaltsanalyse. Forum: Qualitative Sozialforschung 1/2, Art. 20. Online: http://www.qualitative-research.net/index.php/fqs/article/view/108 9/2384 (letzter Aufruf [28.02.2011]).

Mayring, Philipp (2001). Kombination und Integration qualitativer und quantitativer Analyse. Forum: Qualitative Sozialforschung 2/1, Art. 6 Online: http://www.qualitativeresearch. net/index.php/fqs/article/view/967/2111 (letzter Aufruf [12.03.2011]). 
Reinfried, Marcus (1992). Das Bild im Fremdsprachenunterricht: Eine Geschichte der visuellen Medien am Beispiel des Französischunterrichts. Tübingen: Narr.

Rymarczyk, Jutta (2007). Zum Wechselspiel von Text und Bildender Kunst in einer intermedialen Literatur- und Kulturdidaktik. In: Hallet, Wolfgang / Nünning, Ansgar (Hrsg.): Neue Ansätze und Konzepte der Literatur- und Kulturdidaktik. Trier: Wissenschaftlicher Verlag Trier. 329-350.

Seidl, Monika (Hrsg.) (2007). Visual literacy: Bilder verstehen. Themenheft von Der Fremdsprachliche Unterricht Englisch 41/87.

Thomson, Katrin (2010). Bildkunst und Literatur im EFL-Classroom: Überlegungen zur Förderung von Lesekompetenzen durch die bildgestützte Behandlung literarischer Texte. In: Hecke, Carola / Surkamp, Carola (Hrsg.): Bilder im Fremdsprachenunterricht: Neue Ansätze, Kompetenzen und Methoden. Tübingen: Narr. 199-224.

Uhlig, Bettina (2005). Kunstrezeption in der Grundschule: Zu einer grundschulspezifischen Rezeptionsmethode. München: kopaed. (Kontext Kunstpädagogik, Bd. 3.)

Witzel, Andreas (2000). Das problemzentrierte Interview. Forum: Qualitative Sozialforschung 1/1, Art. 22. Online: http://www.qualitative-research.net/index.php/ fqs/article/ view/1132/2520 (letzter Aufruf [11.03.2011]). 



\section{Autorinnen und Autoren}

Dr. Ulrike Arras

Testentwicklung

TestDaF-Institut / g.a.s.t.

Gesellschaft für Akademische

Studienvorbereitung und Testentwicklung e.V.

Massenbergstraße 13b

44787 Bochum

ulrike.arras@testdaf.de

Jan-Oliver Eberhardt

Studienrat

Oberrhein-Gymnasium Weil am Rhein

Königsberger Straße 9

79576 Weil am Rhein

jano.eberhardt@gmx.de

Prof. Dr. Michaela Gläser-Zikuda

Friedrich-Schiller-Universität Jena

Institut für Erziehungswissenschaft Lehrstuhl

für Schulpädagogik und Didaktik

Am Planetarium 4

07749 Jena

michaela.glaeser-zikuda@uni-jena.de

Petra Knorr

Universität Leipzig

Institut für Anglistik

Beethovenstr. 15

04109 Leipzig

pknorr@uni-leipzig.de

Anna Katharina Schnell

Universität Bremen

Fachbereich 10

Angewandte Linguistik / Sprachlehrlernforschung aschnell@uni-bremen.de
Annegret Beier

DAAD-Lektorin

Staatliche Medizinische Universität

Turkmenistans

Archabil shayoly 18

744036 Aschgabat

Turkmenistan

annegret_beier@yahoo.de

Diana Feick

Universität Leipzig

Herder-Institut

Beethovenstraße 15

04107 Leipzig

Prof. Dr. Lena Heine

Juniorprofessorin am Seminar für

Sprachlehrforschung

Ruhr-Universität Bochum

Fakultät für Philologie

Seminar für Sprachlehrforschung

D-44780 Bochum

lena.heine@rub.de

Michael Rogge, OStR i.H.

Ruhr-Universität Bochum

Englisches Seminar

Didaktik des Englischen

Universitätsstr. 150

44801 Bochum

michael.rogge@rub.de 


\section{Diskutantinnen und Diskutanten}

Ruth-Ulrike Deutschmann, M.A.

Technische Universität Dresden

Fakultät Sprach-, Literatur- und

Kulturwissenschaften

Institut für Germanistik

Professur Deutsch als Fremdsprache

Zeunerstraße $1 b$

01069 Dresden

ulrike.deutschmann@mailbox.tu-dresden.de

Prof. Dr. Susanne Göpferich

Justus-Liebig-Universität Gießen

Institut für Anglistik / ZfbK

Karl-Glöckner-Str. 5A

35394 Gießen

Susanne.Goepferich@anglistik.uni-giessen.de

Dr. Henning Rossa

Technische Universität Dortmund

Fachdidaktik Englisch / Angewandte

Linguistik

Institut für Anglistik und

Amerikanistik

Fakultät Kulturwissenschaften

44221 Dortmund

henning.rossa@tu-dortmund.de

Jun. Prof. Dr. Eva Wilden

Ruhr-Universität Bochum

Englisches Seminar

Universitätsstraße 150

44780 Bochum

eva.wilden@rub.de

\author{
Monika Geist \\ Ludwig-Maximilians-Universität \\ München \\ Institut für Englische Philologie \\ Schellingstraße 3 \\ 80799 München \\ monika.geist@lmu.de
}

Dr. Adelheid $\mathrm{Hu}$

Universität Luxemburg

Fakultät für Sprachwissenschaften

und Literatur, Geisteswissenschaften, Kunst und Erziehungswissenschaften

Route de Diekirch

L-7220 Walferdange

adelheid.hu@uni.lu

Prof. Dr. Karen Schramm

Universität Leipzig

Herder-Institut

Deutsch als Fremdsprache

Didaktik/Methodik

Beethovenstraße 15

04107 Leipzig

karen.schramm@uni-leipzig.de 


\section{Kolloquium Fremdsprachenunterricht}

Herausgegeben von Daniela Caspari, Lars Schmelter, Karin Vogt und Nicola Würffel

Kolloquium Fremdsprachenunterricht (KFU) publiziert Tagungsdokumentationen und thematisch ausgerichtete Sammelbände sowie einschlägige Monographien, Dissertationen und Habilitationsschriften zu allen relevanten Fragenstellungen der Fremdsprachenforschung. Insbesondere folgende Forschungsgebiete sind von Interesse: Fremdsprachendidaktik aller Sprachen, Fremdsprachenlehr- und lernforschung, Forschungsmethoden, Mehrsprachigkeitsforschung, interkultureller Fremdsprachenunterricht, Multiliteralitätsforschung, bilingualer Unterricht, mediengestütztes Fremdsprachenlernen und -lehren, Literatur- und Textdidaktik.

Publikationsanfragen richten Sie bitte an eine/n der Herausgeberlnnen, Prof. Dr. Daniela Caspari, Prof. Dr. Lars Schmelter, Prof. Dr. Karin Vogt, Prof. Dr. Nicola Würffel. Gerne berät das Herausgeberteam interessierte Autor/inn/en bei Forschungsprojekten.

Prof. Dr. Daniela Caspari: http://www.geisteswissenschaften.fu-berlin.de/we05/mitarbeiter/caspari/ index.html

Prof. Dr. Lars Schmelter: http://www.romanistik.uni-wuppertal.de/personal/fachdidaktik/prof-dr-phillars-schmelter.html

Prof. Dr. Karin Vogt: http://www.ph-heidelberg.de/englisch/personen/lehrende/prof-dr-vogt.html

Prof. Dr. Nicola Würffel: http://www.ph-heidelberg.de/mediendidaktik/personen/lehrende/prof-dr-nicolawuerffel.html

Band 1 Volker Raddatz / Michael Wendt (Hrsg.): Textarbeit im Fremdsprachenunterricht - Schrift, Film, Video. Kolloquium zur Ehren von Bertolt Brandt (Verlag Dr. Kovac 1997).

Band 2 Gabriele Blell/Wilfried Gienow (Hrsg.): Interaktion mit Texten, Bildern, Multimedia im Fremdsprachenunterricht (Verlag Dr. Kovac 1998).

Band 3 Renate Fery / Volker Raddatz (Hrsg.): Lehrwerke und ihre Alternativen. 2000.

Band 4 Gisèle Holtzer / Michael Wendt (éds.): Didactique comparée des langues et études terminologiques. Interculturel - Stratégies - Conscience langagière. 2000.

Band 5 Gerhard Bach/Susanne Niemeier (Hrsg.): Bilingualer Unterricht. Grundlagen, Methoden, Praxis, Perspektiven. 5., überarbeitete und erweiterte Auflage. 2010.

Band 6 Michael Wendt (Hrsg.): Konstruktion statt Instruktion. Neue Zugänge zu Sprache und Kultur im Fremdsprachenunterricht. 2000.

Band 7 Dagmar Abendroth-Timmer/Stephan Breidbach (Hrsg.): Handlungsorientierung und Mehrsprachigkeit. Fremd- und mehrsprachliches Handeln in interkulturellen Kontexten. 2000.

Band 8 Wolfgang Zydatiß: Leistungsentwicklung und Sprachstandserhebungen im Englischunterricht. Methoden und Ergebnisse der Evaluierung eines Schulversuchs zur Begabtenförderung: Gymnasiale Regel- und Expressklassen im Vergleich. Unter Mitarbeit von Viola Vockrodt-Scholz. 2002.

Band 9 Wilma Melde / Volker Raddatz (Hrsg.): Innovationen im Fremdsprachenunterricht 1. Offene Formen und Frühbeginn. 2002.

Band 10 Gerhard Bach / Britta Viebrock (Hrsg.): Die Aneignung fremder Sprachen. Perspektiven Konzepte - Forschungsprogramm. 2002.

Band 11 Hannelore Küpers / Marc Souchon (Eds.): Appropriation des Langues au Centre de la Recherche. Spracherwerb als Forschungsgegenstand. 2002. 
Band 12 Helene Decke-Cornill / Maike Reichart-Wallrabenstein (Hrsg.): Fremdsprachenunterricht in medialen Lernumgebungen. 2002.

Band 13 Nikola Mayer: Ganzheitlichkeit und Sprache. Theorie des Begriffs und empirische Zugangswege im Gespräch mit Fremdsprachenlehrerinnen- und -lehrern. 2002.

Band 14 Brigitte Krück/Christiane Loeser (Hrsg.): Innovationen im Fremdsprachenunterricht 2. Fremdsprachen als Arbeitssprachen. 2002.

Band 15 Johannes Eckerth / Michael Wendt (Hrsg.): Interkulturelles und transkulturelles Lernen im Fremdsprachenunterricht. 2003.

Band 16 Dagmar Abendroth-Timmer/Britta Viebrock/Michael Wendt (Hrsg.): Text, Kontext und Fremdsprachenunterricht. Festschrift für Gerhard Bach zum 60. Geburtstag. 2003.

Band 17 Petra Bosenius/Jürgen Donnerstag (Hrsg.): Interaktive Medien und Fremdsprachenlernen. 2004.

Band 18 Mercedes Díez / Raquel Fernández / Ana Halbach (eds.): Debate en torno a las estrategias de aprendizaje. Debating Learning Strategies. 2004.

Band 19 Adelheid Schumann (Hrsg.): Kulturwissenschaften und Fremdsprachendidaktik im Dialog. Perspektiven eines interkulturellen Französischunterrichts. 2005.

Band 20 Christine Penman (ed.): Holistic Approaches to Language Learning. 2005.

Band 21 Steffi Morkötter: Language Awareness und Mehrsprachigkeit. 2005.

Band 22 Wolfgang Zydatiß: Bildungsstandards und Kompetenzniveaus im Englischunterricht. Konzepte, Empirie, Kritik und Konsequenzen. 2005.

Band 23 Gerhard Bach/Gisèle Holtzer (éds.): Pourquoi apprendre des langues? Orientations pragmatiques et pédagogiques. 2006.

Band 24 Andreas Grünewald: Multimedia im Fremdsprachenunterricht. Motivationsverlauf und Selbsteinschätzung des Lernfortschritts im computergestützten Spanischunterricht. 2006.

Band 25 Christiane Fäcke: Transkulturalität und fremdsprachliche Literatur. Eine empirische Studie zu mentalen Prozessen von primär mono- oder bikulturell sozialisierten Jugendlichen. 2006.

Band 26 Laurence Jeannot: Introduction des TICE en contexte scolaire et autonomie dans l'apprentissage des langues étrangères. Stratégies et compétence d'apprentissage dans des dispositifs mixtes en France et en Allemagne. 2006.

Band 27 Helmut Johannes Vollmer (Hrsg.): Synergieeffekte in der Fremdsprachenforschung. Empirische Zugänge, Probleme, Ergebnisse. 2007.

Band 28 Jean E. Conacher/Helen Kelly-Holmes (eds.): New Learning Environments for Language Learning. Moving beyond the classroom? 2007.

Band 29 Daniela Caspari/Wolfgang Hallet/Anke Wegner/Wolfgang Zydatiß (Hrsg.): Bilingualer Unterricht macht Schule. Beiträge aus der Praxisforschung. 2., durchgesehene Auflage. 2009.

Band 30 Sabine Doff/Torben Schmidt (Hrsg.): Fremdsprachenforschung heute. Interdisziplinäre Impulse, Methoden und Perspektiven. 2007.

Band 31 Daniela Elsner/Lutz Küster/Britta Viebrock (Hrsg.): Fremdsprachenkompetenzen für ein wachsendes Europa. Das Leitziel „Multiliteralität“. 2007.

Band 32 Adelheid Schumann / Lieselotte Steinbrügge (Hrsg.): Didaktische Transformation und Konstruktion. Zum Verhältnis von Fachwissenschaft und Fremdsprachendidaktik. 2008.

Band 33 Dagmar Abendroth-Timmer: Akzeptanz und Motivation. Empirische Ansätze zur Erforschung des unterrichtlichen Einsatzes von bilingualen und mehrsprachigen Modulen. 2007. 
Band 34 Christine Hélot / Britta Benert/ Sabine Ehrhart / Andrea Young (éds.): Penser le bilinguisme autrement. 2008.

Band 35 Christiane Fäcke (Hrsg.): Sprachbegegnung und Sprachkontakt in europäischer Dimension. 2009.

Band 36 Dagmar Abendroth-Timmer/Daniela Elsner/Christiane Lütge/Britta Viebrock (Hrsg.): Handlungsorientierung im Fokus. Impulse und Perspektiven für den Fremdsprachenunterricht im 21. Jahrhundert. 2009.

Band 37 Karin Aguado/Karen Schramm/Helmut Johannes Vollmer (Hrsg.): Fremdsprachliches Handeln beobachten, messen, evaluieren. Neue methodische Ansätze der Kompetenzforschung und der Videographie. 2010.

Band 38 Bernd Tesch: Kompetenzorientierte Lernaufgaben im Fremdsprachenunterricht. Konzeptionelle Grundlagen und eine rekonstruktive Fallstudie zur Unterrrichtspraxis (Französisch). 2010.

Band 39 Maria Giovanna Tassinari: Autonomes Fremdsprachenlernen. Komponenten, Kompetenzen, Strategien. 2010.

Band 40 Daniela Caspari/Lutz Küster (Hrsg.): Wege zu interkultureller Kompetenz. Fremdsprachendidaktische Aspekte der Text- und Medienarbeit. 2010.

Band 41 Dagmar Abendroth-Timmer / Marcus Bär / Bàrbara Roviró / Ursula Vences (Hrsg.): Kompetenzen beim Lernen und Lehren des Spanischen. Empirie und Methodik. 2011.

Band 42 Daniela Elsner/Anja Wildemann (Hrsg./eds.): Sprachen lernen - Sprachen lehren. Language Learning - Language Teaching. Perspektiven für die Lehrerausbildung in Europa. Prospects for Teacher Education across Europe. 2011.

Band 43 Paula Krüger: Sprachlernhabitus und Bedeutungskonstruktion beim Fremdsprachenlernen. 2011.

Band 44 Lena C. Bellingrodt: ePortfolios im Fremdsprachenunterricht. Empirische Studien zur Förderung autonomen Lernens. 2011.

Band 45 Urška Grum: Mündliche Sprachkompetenzen deutschsprachiger Lerner des Englischen. Entwicklung eines Kompetenzmodells zur Leistungsheterogenität. 2012.

Band 46 Susanne Staschen-Dielmann: Narrative Kompetenz im bilingualen Geschichtsunterricht. Didaktische Ansätze zur Förderung der schriftlichen Diskursfähigkeit. 2012.

Band 47 Corinna Koch: Metaphern im Fremdsprachenunterricht: Englisch, Französisch, Spanisch. 2013.

Band 48 Karin Aguado / Lena Heine / Karen Schramm (Hrsg.): Introspektive Verfahren und Qualitative Inhaltsanalyse in der Fremdsprachenforschung. 2013.

www.peterlang.de 